\title{
Enlightening the Dark
}

\author{
Anastasia Sokolenko
}

Thesis submitted for the degree of Philosophiae Doctor

Supervisor: Torsten Bringmann

September 2019 
(C) Anastasia Sokolenko, 2019

Series of dissertations submitted to the

Faculty of Mathematics and Natural Sciences, University of Oslo No. 2173

ISSN 1501-7710

All rights reserved. No part of this publication may be reproduced or transmitted, in any form or by any means, without permission.

Print production: Reprosentralen, University of Oslo. 
To mysterious Dark Matter. 



\section{Abstract}

The Standard Model (SM) of particle physics is extremely successful experimentally - it has passed countless tests at accelerators, all its main predictions have been confirmed to very high precision. The last prediction of the SM, the Higgs boson, was discovered at the Large Hadron Collider (LHC) in 2012 [1, 2].

Yet we know now that it is not complete. The main reason to believe so is based on the existence of several observed phenomena that cannot be incorporated into the Standard Model. These phenomena include:

- Neutrino masses: Why do neutrinos have mass?

- Dark Matter: What is the prevalent kind of matter in the Universe?

- Baryon asymmetry of the Universe: What mechanism had created a tiny matterantimatter imbalance in the Early Universe?

The research described in this thesis is motivated by the necessity to find resolutions to these problems. We concentrate on the problem of Dark Matter (DM), however, the other problems, as well as deep theoretical questions raised by the properties of the SM, are used as a source of motivation for the models of new physics containing DM candidates.

We start by presenting the evidence for the existence of DM and stress that these evidence come both from the observed properties of a large number of astrophysical objects of different sizes and nature and from the properties of our Universe as a whole. These evidence are also obtained using several very different and independent observational methods. This makes the existence of DM as a phenomenon solidly confirmed. The hypothesis that DM is made of particles is less established but seems to be rather plausible. Alternative models either have difficulties to explain the whole body of evidence in favor of DM, or are strongly constrained experimentally. If the assumption of DM particle appears to be true, this particle cannot be a part of the Standard Model.

We briefly describe what is known about possible properties of DM particle and give examples of potential particle physics candidates. We proceed discussing in details one particular scenario for DM - self-interacting DM. We have reviewed astrophysical constraints on the cross-section of the self-interacting DM using data from observed objects of different sizes (over 7 orders of magnitude in the total mass, from dwarf galaxies to galaxy clusters). We systematically tested semi-analytic models used in the literature to make predictions for the DM density cores and concluded that to obtain reliable bounds it is important to use directly the data produced by realistic Self-interacting Dark Matter (SIDM) simulations. To demonstrate this we have collected ensembles of haloes of all observed 
sizes simulated with the velocity-independent SIDM. These simulations were performed including many effects of baryonic matter that can affect the properties of DM haloes and observable quantities. This allows making robust predictions that can be directly compared with observations. The robust constraints obtained here provide important input for the particle physics models of SIDM. We later discuss how these constraints can be combined (for a particular model) with the constraints from particle physics experiments.

Another aspect of Dark Matter physics is its indirect detection. To this end, we have analyzed the sensitivity of Cherenkov Telescope Array (CTA) for the signal from the annihilation of DM particles. We have tested the software package ctools adopted by the CTA collaboration for the detection of a signal with corresponding morphology. Although the treatment of systematic errors is not yet implemented in ctools, they can be taken into account adopting a simple physical approach that does not require code modifications. We demonstrate that assuming an optimistic Einasto profile for the DM density distribution in the Milky Way and $W W$ annihilation channel, with 500 hours of the Galactic Center observations CTA can probe the annihilation cross-section below its thermal value.

Finally, we considered in details an example of combinations of different constraints, coming both from particle physics on one side and from cosmology and astrophysics on the other side. We have considered a simple model that contains a neutral fermion playing the role of DM and a scalar that mixes with the Higgs boson and mediates the interaction of DM with the Standard Model. We concentrated on the case of light DM fermion, such that traditional direct detection approaches are difficult to apply as the recoil of nuclei becomes weak. We also assumed that the mediator is lighter than DM, such that it can decay visibly at the accelerators and give rise to an interesting astrophysical phenomenology. We have shown that the constraints from accelerator searches for light scalars, when combined with the astrophysical constraints on DM self-interaction (or with the requirement of the correct DM abundance assuming thermal production) appear to be very strong. Future intensity frontier experiments (such as e.g. SHiP) may improve this even further and allow to probe the part of the parameter space of the model that, in terms of direct detection experiments, reaches the irreducible neutrino background.

In this thesis, our main motivation originated from the DM problem. Model-independent or widely applicable results remain a necessary ingredient for identifying a successful complete extension of the Standard Model. Of course, it is very important to apply the astroparticle approach, combining model-independent constraints of different nature, to more realistic models that can explain not only DM but also neutrino masses and matterantimatter asymmetry of the Universe. This ambitious goal, however, can be achieved only for very specific models. Steven Weinberg wrote in his famous paper of 1967: "Of course our model has too many arbitrary features for these predictions to be taken very seriously...". However, his theory appeared to be the correct model of elementary particles that is definitely a great victory of human Science! 


\section{Acknowledgments}

Foremost, I would like to express my sincere gratitude to my mentor Torsten Bringmann, who put quite a lot of efforts in order to give me an opportunity to do my Ph.D. in Oslo and taught me to be independent in my research as well as the freedom to explore my ideas. Thank you very much for your support and patience in all academic and non-academic matters, and for your calmness and peacefulness.

I am deeply grateful to my teachers Alexey Boyarsky and Kyrylo Bondarenko, for your support and encouragement in my development as a scientist. Thank you very much for our endless discussions from dawn to dusk.

My personal thanks go out to my colleagues at the University of Oslo and at the Lorentz Institute. I express my gratitude to my collaborators Thejs Brinckmann, Lesya Shchutska, Gabrijela Zaharijas, Christopher Eckner, and Lili Yang. Also, I would like to thank Denys Malyshev, Oleg Ruchayskiy, Andrii Magalich, Andrzej Jan Hryczuk, Ana Achúcarro, and Stanislav Vilchynskyi for interesting and fruitful discussions as well as their help. I thank my friends Anton, Timo, Iryna, Alex, and Akram for your interaction which has changed me a little for the better and enriched my life.

Last but not least, special thanks go to my partner Thomas, for sharing beautiful moments together and keeping me happy. I deeply thank my family, my mother Iryna, for her tremendous support throughout my life and for her love, which is always a warm home in my heart in any joyful and tough moments. Also, I would not be here without my father Oleg, thank you for your understanding and for your help in becoming who I am now. My dear brother Dmitry, thanks for having cheered me on since I was little girl, and walking up the path in front of me. 


\section{List of publications}

The thesis is based on the following publications:

Paper I K. Bondarenko, A. Boyarsky, T. Bringmann, and A. Sokolenko, Constraining self-interacting dark matter with scaling laws of observed halo surface densities, JCAP 1804 (2018) no.04, 049, [arXiv:1712.06602]

Paper II A. Sokolenko, K. Bondarenko, T. Brinckmann, J. Zavala, M. Vogelsberger, T. Bringmann, and A. Boyarsky, Towards an improved model of self-interacting dark matter haloes, JCAP 1812 (2018) no.12, 038, [arXiv:1806.11539]

Paper III I. Boiarska, K. Bondarenko, A. Boyarsky, V. Gorkavenko, M. Ovchynnikov, and A. Sokolenko, Phenomenology of GeV-scale scalar portal, [arXiv:1904.10447]

In addition, this thesis contains work that will be part of the following upcoming CTA consortium publication (manuscript in preparation):

Paper IV CTA Consortium (T. Bringmann et al.), Pre-construction estimates of the CTA sensitivity to a dark matter signal from the Galactic centre, 2019, manuscript in preparation 


\section{Acronyms}

DM Dark Matter

CTA Cherenkov Telescope Array

VHE Very High Energy

IACTs Imaging Atmospheric Cherenkov Telescopes

LST Large Sized Telescope

MST Medium Sized Telescope

SST Small Sized Telescope

SM Standard Model

WIMP Weakly interacting massive particle

CR Cosmic Ray

GDE Galactic Diffuse Emission

NFW Navarro-Frenk-White

SIDM Self-interacting Dark Matter

CDM Cold Dark Matter

dSphs Dwarf Spheroidal Galaxies

CCCP Canadian Cluster Comparison Project

CMB Cosmic microwave background

MACHOs Massive Astrophysical Compact Halo Objects

PBHs Primordial Black Holes

MOND Modified Newtonian dynamics

CDM Cold Dark Matter 
WDM Warm Dark Matter

HDM Hot Dark Matter

WIMP Weakly Interacting Massive Particle

ALP Axion-like particle

LHC Large Hadron Collider

BAU Baryon Asymmetry of the Universe 


\section{Contents}

1 Introduction 1

2 Dark matter: Cold Dark Matter and its alternatives 7

2.1 Evidence of Dark Matter . . . . . . . . . . . . . . . 7

2.2 The Nature of Dark Matter . . . . . . . . . . . . . . . . . . . . . . . . . . . . . . . . 13

2.3 Properties of Dark Matter particles . . . . . . . . . . . . . . . . 16

2.4 Examples of models for DM particles . . . . . . . . . . . . . 21

2.5 Dark Matter detection . . . . . . . . . . . . . . . . . . 23

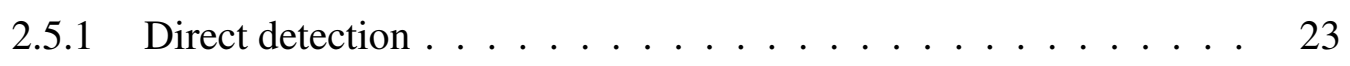

2.5 .2 Indirect detection . . . . . . . . . . . . . . . . 25

2.5.3 Search at accelerators . . . . . . . . . . . . 31

3 Self-interacting Dark Matter $\quad 33$

3.1 Theoretical description of SIDM . . . . . . . . . . . . 33

3.1.1 Qualitative picture: local equilibrium and $r_{\text {SIDM }} \ldots \ldots 33$

3.1.2 Analytic description of SIDM haloes . . . . . . . . . . . . 35

3.2 Testing the theory with simulations . . . . . . . . . . . . . 38

3.2.1 Main properties of simulated SIDM haloes . . . . . . . . . 39

3.2.2 Testing the model assumptions by N-body simulations . . . . . . 40

3.2.3 Testing predictions for the cores . . . . . . . . . . . . 42

3.2.4 Anisotropic velocities and improved prediction for the inner density 44

3.2.5 Predictions based on NFW fits . . . . . . . . . . . . . 46

3.2.6 Predicting the radius $r_{M}$ for a given cross-section . . . . . . . . 47

3.2.7 Connecting $r_{\text {core }}$ and $r_{\text {SIDM }}$ by fitting rotation curve or density profile 50

3.2.8 Surface density and model-dependent constraints on SIDM . . . . 52

3.2.9 DM surface density in SIDM halo: semi-analytic predictions . . . 55

3.2.10 Statistical constraints from the surface density data and semi-analytic predictions ......................... 56

3.3 Direct comparison of simulations with observations . . . . . . . . . 59

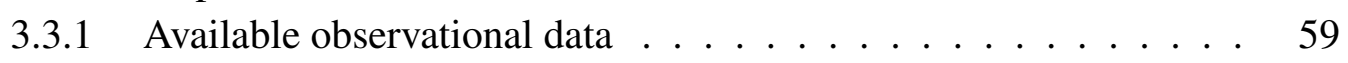

3.3 .2 SIDM in simulations . . . . . . . . . . . . . 63

3.3.3 Comparison of the surface density between data and simulations . 69

3.4 Conclusions . . . . . . . . . . . . . . . . . 71 
4 Indirect detection of Dark Matter with Imaging Atmospheric Cherenkov telescopes

4.1 Imaging Atmospheric Cherenkov telescopes . . . . . . . . . . . . . . 72

4.1.1 Cherenkov radiation . . . . . . . . . . . . 73

4.1 .2 Air Showers . . . . . . . . . . . . . . . 76

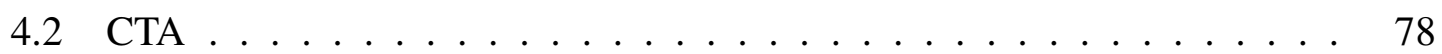

4.3 CTA simulations and data analysis with ctools . . . . . . . . . . 80

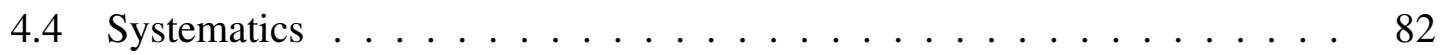

4.5 Sensitivity for DM annihilation from the Galactic Center and comparison with other methods . . . . . . . . . . . . . . . . 85

4.5.1 Uncertainties in the expected signal from the Galactic Center . . . 86

4.5.2 Calculation of sensitivity . . . . . . . . . . . . . 88

4.6 Conclusion . . . . . . . . . . . . . . . . . . . 90

5 Light Dark Matter with a scalar mediator $\quad 92$

5.1 Introduction . . . . . . . . . . . . . . . . . . . . 92

5.2 Model description . . . . . . . . . . . . . . . . . . . . . . . . . . . . . . . . . . 93

5.3 Dark matter self-interaction . . . . . . . . . . . . . . . . 95

5.4 Constraints from accelerator searches _ . . . . . . . . . . . . . 98

5.4.1 Accelerator phenomenology of the scalar portal . . . . . . . . 98

5.4.2 Experiments constraining scalar portal . . . . . . . . . . . . 99

5.4.3 Constraints from the invisible Higgs decay . . . . . . . . . . . 103

5.5 Direct detection . . . . . . . . . . . . . . . . . . . . . 104

5.6 Conclusions . . . . . . . . . . . . . . . . . . . 107

$\begin{array}{llr}6 & \text { Conclusions and outlook } & 108\end{array}$

$\begin{array}{llr}7 & \text { Papers } & 112\end{array}$

Paper I . . . . . . . . . . . . . . . . . . . . . . . . . . . . . . . . . 113

Paper II . . . . . . . . . . . . . . . . . . . . . . . . 142

Paper III . . . . . . . . . . . . . . . . . . . . . . . . . . . . . . . 174

$\begin{array}{ll}\text { A Jeans equation } & 225\end{array}$

B Reconstructing the inner SIDM density from NFW fit obtained at large distances

C Parametric models for the data $\quad 229$

$\begin{array}{ll}\text { Bibliography } & \mathbf{2 3 1}\end{array}$ 


\section{Chapter 1}

\section{Introduction}

The Standard Model of particle physics. The long development of particle physics in the previous century resulted in the construction of the SM of particle physics. Starting from a phenomenological description of radioactivity and nuclear interactions, particle physics predicted and discovered many new particles. Some of the new discoveries came instead completely unexpectedly - the existence of three generations that in many aspects simply repeat each other, the parity and then $C P$ violation.

The SM, that takes into account all these phenomenologies in a minimal and consistent way, is an extremely successful theory. It has passed a very large number of experimental checks and cross-checks. The Standard Model has also performed very well in the precision tests where its predictions were tested not only to the leading order but also to next or to next-to-next leading order. The last prediction of the SM, the Higgs boson, was discovered at the LHC in 2012 [1, 2]. In the period from 2012 till 2018 LHC was successfully exploring all the production and decay products of the Higgs boson that exist in the Standard Model [3]. These results have confirmed that the discovered particle is indeed the SM Higgs boson, not just a Higgs-like particle.

This means that the Standard Model provides a complete and closed description of particle physics as observed at accelerators. It is also a mathematically consistent theory that can be valid up to very high energies, probably up to the Planck scale (see e.g. [4] and references therein).

Nevertheless, we know today that the SM is not the final model of Nature and some new physics should exist.

The main reason to believe so is based on the existence of several observed phenomena that can not be incorporated into the Standard Model. These phenomena include:

- Neutrino masses: Why do neutrinos have mass which is prohibited within the Standard Model?

- Dark Matter: What is the prevalent kind of matter in the Universe? 
- Baryon asymmetry of the Universe: What mechanism had created a tiny matterantimatter imbalance in the Early Universe?

This list may turn out to be incomplete - there are more experimental anomalies which, if confirmed, will not be consistent with the Standard Model. There are deep questions in cosmology that may require new physics for their understanding, as well as theoretical questions and the peculiar features of the Standard Model that may point out to some physics beyond it. However, first of all, physics is an experimental science. Let us first concentrate therefore on the confirmed observational phenomena listed above.

Neutrino masses. The neutrino was initially discovered as a very light particle [5]. Experimentally, the mass of neutrinos was consistent with zero for many decays. These data were consistent with the Standard Model until numerous observations of neutrino flavor oscillations have put a lower bound on the masses of at least two neutrinos [6,7]. Currently, we can say that it is already very well established experimentally that at least two neutrino species are massive.

Indeed, in the SM the number of leptons is independently conserved for each flavor. This property is confirmed in all processes observed at accelerators. However, numerous experiments demonstrate that neutrinos propagating from the Sun to Earth (for a review see e.g. [8]), from higher layers of the atmosphere to the ground (for a review see e.g. [9]) and from nuclear reactors to remote detectors (for a review see e.g. $[10,11]$ ) can "oscillate" i.e. can change their flavour with a probability that periodically changes with the distance from the source. This phenomenon can be easily explained for all three flavors if neutrinos have different masses (for a review see e.g.[12]). The period of oscillations between neutrinos of two flavors is proportional to the squares of difference in their masses [13]. Experimentally two mass differences are observed [14]

$$
\begin{aligned}
\Delta m_{\text {solar }}^{2} & =(7.53 \pm 0.18) \cdot 10^{-5} \mathrm{eV}^{2}, \\
\Delta m_{\mathrm{atm}}^{2} & =(2.51 \pm 0.05) \cdot 10^{-3} \mathrm{eV}^{2} .
\end{aligned}
$$

This means that at least two of the three neutrinos are massive (the lightest one is still allowed to be massless by the data). These three masses can also be organized into the so-called normal and inverse mass hierarchies, see Fig. 1.1.

On the other hand, it is not possible to incorporate neutrino masses into the Standard Model without adding some new physics that has not been observed yet. This is related to the gauge symmetries of the SM. Indeed, the usual Dirac mass is defined for a fermion as

$$
\mathcal{L}_{m}=m_{d} \bar{\psi}_{L} \psi_{R}+\text { h.c. }
$$

For neutrinos, only the left-handed states were experimentally discovered [16]. To write the Dirac mass we need to assume the existence of right-handed neutrinos. By 

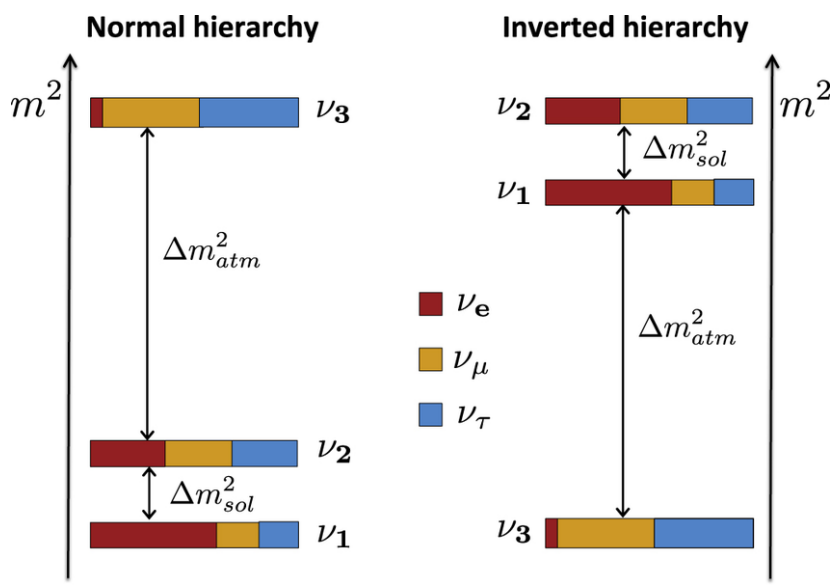

Figure 1.1: Normal and inverse mass hierarchies for neutrinos. Colours indicate neutrino flavours. Taken from [15].

the structure of the Standard Model, these particles should be gauge singlets and naively would not interact with the SM. However, as neutrinos are massive, they can interact via mixing with the usual left-handed counterparts and their phenomenology can be quite rich, especially in the Early Universe (see e.g [17]). In any case, right-handed neutrinos are already new physics and we do not know for sure if they exist and if they do, what are their masses, couplings and other properties?

In the absence of right-handed neutrinos, we can write a Majorana mass term for lefthanded neutrinos. Experimentally, it is not known whether neutrinos are Dirac or Majorana particles (see [18] for a recent overview of the corresponding experimental activities). Theoretically, a Majorana mass for left-handed SM neutrinos also requires new physics. Indeed, fermions in the SM are organized in left-handed doublets and right-handed singlets:

$$
L=\left(\begin{array}{c}
\nu_{L} \\
e_{L}
\end{array}\right), \quad R=e_{R}
$$

For the Higgs field vacuum expectation value in the form

$$
H_{0}=\left(\begin{array}{c}
0 \\
v / \sqrt{2}
\end{array}\right)
$$

one gets

$$
e_{L}=\frac{\sqrt{2}}{v}\left(H_{0}^{\dagger} L\right), \quad \nu_{L}=-\frac{\sqrt{2}}{v}\left(H_{0}^{\dagger} i \sigma_{y} L\right) .
$$

We can use this to build a gauge invariant Majorana mass for the left-handed neutrinos:

$$
\mathcal{L}_{m, \nu}=m_{\nu} \bar{\nu}_{L}\left(\nu_{L}\right)^{c} \equiv \frac{1}{\Lambda}\left(\bar{L} \sigma_{y} H_{0}\right)\left(H_{0}^{\dagger} \sigma_{y} L\right)^{c}
$$


where $\nu_{L}$ is given by Eq. (1.6).

It is easy to see that this operator has mass dimension 5 and, therefore, $\Lambda$ has the dimension of energy. Dimensionful coupling constants (like e.g. the Fermi constant $G_{F}$ ) give rise to unphysical cross-sections that unlimitedly grow with energy and hence indicate the existence of new physics.

We conclude that neutrino masses and flavor oscillations indicate the existence of new physics beyond the Standard Model.

Dark Matter. Dark Matter is another well-established phenomenon that currently has no confirmed explanations. Many experts are convinced that the existence of DM implies some new particle physics beyond the Standard Model. This would give an example of how astronomy and cosmology provide crucial input for particle physics. However, there are attempts to explain DM by e.g. modified Newtonian dynamics or primordial black holes. Such explanations also require some new physics, but not necessarily in terms of particles. DM is the main subject of this thesis, therefore we review the evidence for its existence, its known properties and potential resolutions of the DM puzzle in details in Section 2.

Baryon asymmetry of the Universe. It was already Dirac who predicted in 1928 that charged fermions should have antiparticles [19]. As a particle and an antiparticle annihilate when they meet each other, it is not surprising that we see around us only a very little amount of antiparticles (mainly coming from cosmic rays or created at accelerators). We can see that the Universe as a whole is also very asymmetric in favor of matter over anti-matter. The fraction of antiparticles in cosmic rays is at the level of $10^{-1}-10^{-5}$ (see e.g. [20]). Theoretically, it could be possible that somewhere in the Universe there are regions dominated by anti-matter. However, if such regions existed, then at the boundary between matter and anti-matter dominated regions we would have a huge area of active annihilation. Such a macroscopic source can only remain unnoticed if it is located extremely far away from us, effectively close to the cosmological horizon [21].

Observations show that our Universe does not contain any significant amount of antimatter.

When the Universe was hot, particles and antiparticles could be easily created in pairs. Then, the Universe cooled down, the pairs had to annihilate and we would be left only with photons. To have the state that we observe now, with matter, without antimatter and with baryon to photon ration at the level of $10^{-10}$, we need to assume that the numbers of 
particles and antiparticles were not exactly equal in the hot Universe, before annihilation. For this to happen the so-called Sakharov's conditions have to satisfy [22]:

- Violation of baryon number conservation;

- Breaking of the $C$ and $C P$ symmetries;

- Deviation from thermal equilibrium.

However, as it was firmly established after long studies, in the Standard Model this is not possible. There is no mechanism to generate such an asymmetry in the high-temperature Universe (see e.g. [23] for a review). Therefore, the baryon asymmetry of the Universe was either present in the initial conditions, or was generated later, during the evolution of the hot Universe. In the latter case, some new physics should exist.

The possibility that the asymmetry was present in the initial conditions is very difficult to exclude. However, if the initial conditions for structure formation were generated during the stage of accelerated expansion (cosmic inflation), the asymmetry that existed before inflation would be diluted by at least 60 e-foldings. To give the observed value of the baryon asymmetry of the Universe such a pre-inflationary asymmetry should be huge. We will assume that this did not happen.

This means that the matter-antimatter asymmetry of the Universe also requires some new physics.

Apart from these puzzles, there are other unresolved problems in particle physics and cosmology. It is well established observationally that currently the Universe expands with acceleration [24]. It is not exactly known what drives this accelerated expansion. This is often referred to as the problem of "Dark Energy". However, the current data are consistent with a very minimalistic explanation of Dark Energy - a cosmological constant added to Einstein equations. In this case, no new particles of fields are required to explain "Dark Energy".

In any case, the three experimental problems of the Standard Model listed above strongly suggest that some new physics should exist. Of course, it is possible that each of the above-mentioned challenges has its own explanation, unrelated to the other problems. However, the goal of physics is to build testable theories of nature. It is therefore very attractive to propose a complete theory that could explain all three puzzles within the same framework.

At the same time, we have no firm knowledge about the masses, interaction strength, spin and charges of the new particles responsible for neutrino masses, Dark Matter and generation of the baryon asymmetry in the Universe. After the discovery of the Higgs boson $[1,2]$ the era of "guaranteed discoveries" of particles with predicted properties has 
finished. The main science goal of Tevatron [25, 26] and then LHC [27] was to search for the Higgs boson. Of course, in this situation, it was motivated to search for new physics that could be found together with the Higgs boson, at the same machine. After the results of the LHC Run I and Run II [14], that did not reveal any confirmed signatures of the new physics, it has become even more important to search for new physics in a wider context. A special role is played in this respect by the data from cosmology and astrophysics.

In this thesis, we will mainly concentrate on the problem of Dark Matter. In Section 2 we present the evidence for the existence of Dark Matter, explain why it can not be made of Standard Model particles, discuss its possible properties and several potential particle physics candidates. In Section 3 (based on papers I and II [28, 29]) we discuss in details one particular scenario for Dark Matter - self-interacting DM and possible ways to constrain it from astronomical data. Section 4 (based on paper IV [30]) is devoted to indirect detection of Dark Matter. Section 5 (partially based on paper III [31]) introduces how to search for DM at accelerators and discusses how different ways to constrain the properties of DM particles can be combined together for a particular model. 


\section{Chapter 2}

\section{Dark matter: Cold Dark Matter and its alternatives}

\subsection{Evidence of Dark Matter}

Starting from the first observations by Fritz Zwicky in 1933 [32], who discovered that the mass of the Coma cluster, deduced from its dynamics, was hundred times larger than its luminous mass, the nature of DM remains a mystery. The conclusion that there exists a form of matter which manifests itself only via gravitational interaction was later confirmed by many other observations of various nature.

How do we know that DM exists? Currently, the evidence for the existence of DM consists of two main parts - the astrophysical and the cosmological arguments.

The astrophysical evidence are:

- Rotation curves in spiral galaxies: spiral galaxies (see Fig. 2.1) form in a thin disk of stars and interstellar gas that rotate around its center. One can measure a Doppler shift of some emission or absorption spectral lines from a part of a galaxy and find the velocity of this source with respect to Earth,

$$
\frac{\Delta \lambda}{\lambda}=\frac{V}{c} \equiv \frac{v+U}{c},
$$

where $V$ is the velocity of the emitter/absorber along the line of sight. This velocity has to be spitted into a local velocity $v$ with respect to the center of the galaxy and the velocity of the galaxy movement as a whole, $U$. Averaging this data from different regions of a galaxy we can find $U$ as

$$
\frac{\langle\Delta \lambda\rangle}{\lambda}=\frac{\langle v+U\rangle}{c}=\frac{U}{c}
$$

where $\langle\Delta \lambda\rangle$ is the average of $\Delta \lambda$ by different parts of the galaxy. Subtracting the average velocity $U$ we find the rotational velocity as a function of radius, $v(r)$. Using 


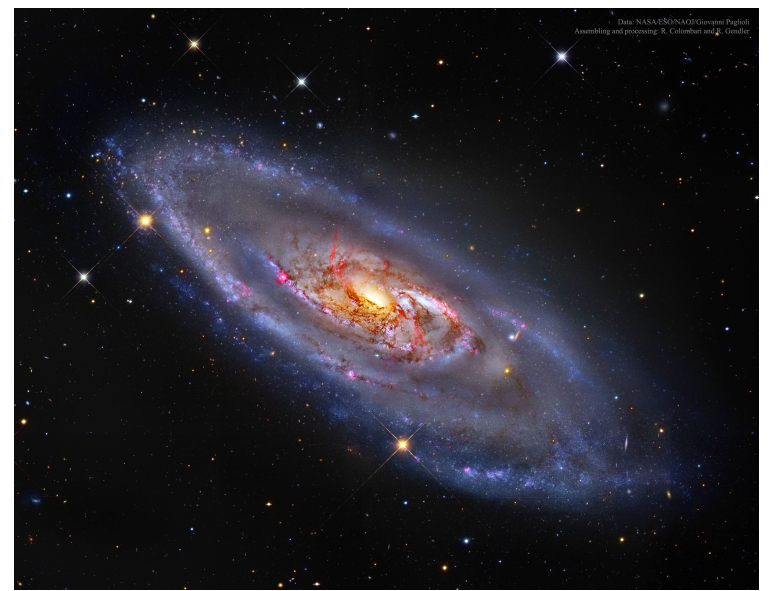

Figure 2.1: A spiral galaxy (M 106) in the constellation Canes Venatici. CRedit: NASA, ESO, NAOJ, Giovanni Paglioli.

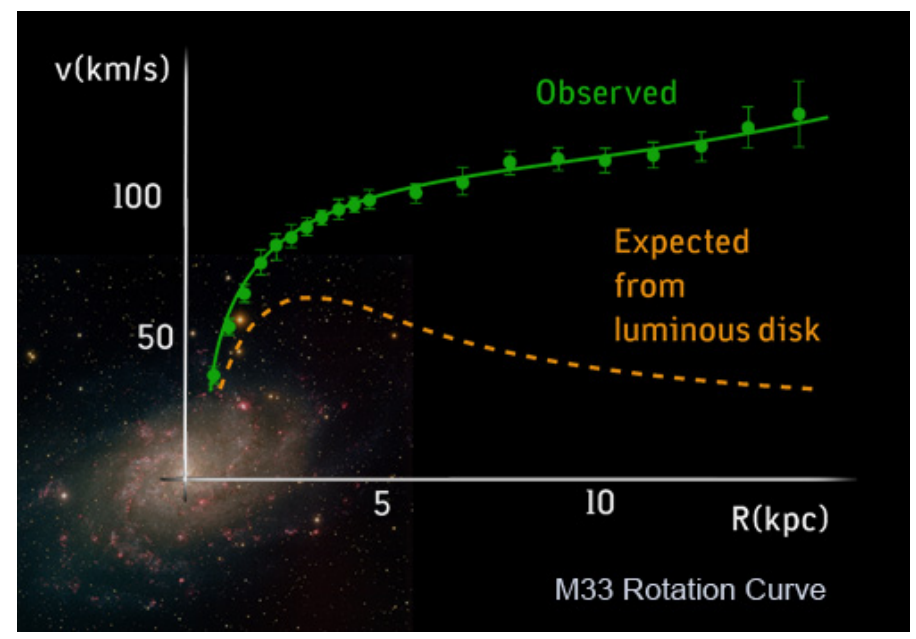

Figure 2.2: The rotation curve for a galaxy M33. Credit: NOAO, AURA, NSF, T.A.Rector.

the rotation velocity one can calculate the total mass

$$
M(r)=\frac{v^{2}(r) r}{G_{N}}
$$

where $G_{N}$ is the Newtonian constant of gravitation. At large radii, where the density of gas and stars is small, the rotation velocity should behave as $v \sim 1 / \sqrt{r}$. However, we do not observe such behavior. Instead, rotation curves of galaxies at large radii typically becomes flat, see an example in Fig. 2.2. At the same time, in the outer part of the galaxy, we do not observe any significant emission or absorption of light, at any wavelength. Therefore, using rotation curves one can conclude that some additional dark (i.e. not interacting with light) mass is needed to explain observational data at large radii. 


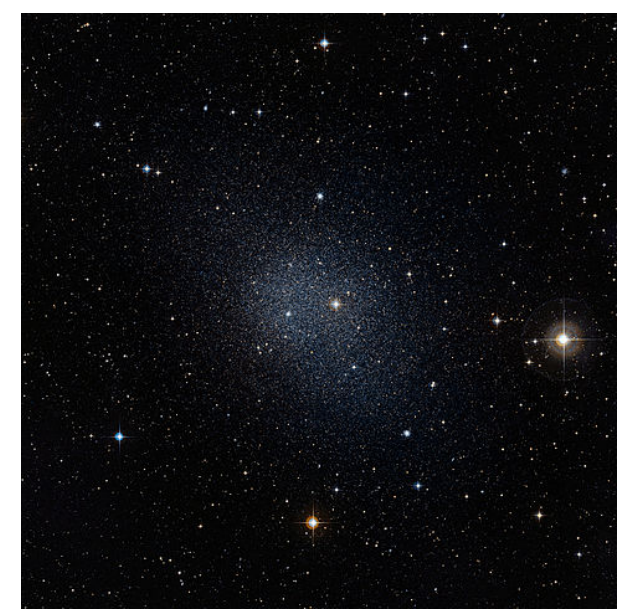

Figure 2.3: The Fornax dwarf galaxy, one of the satellites of the Milky Way. Credit: ESO/Digitized Sky Survey 2.

- Velocity dispersion of stars in dwarf galaxies: Some galaxies do not form disks. Therefore, the rotation velocity cannot be measured and the observable quantity for such objects is the velocity dispersion along the line of sight, $\sigma_{v}$. To reconstruct the mass profile, one needs to solve the Jeans equation that connects the velocity dispersion and density of stars to the total gravitational potential (see Appendix A). The main uncertainty in this method comes from the fact that only the component of the velocity dispersion along the line of sight is directly measured. To reconstruct the full velocity dispersion that is needed for the Jeans equation, we need to assume some anisotropy profile which is unknown. One can marginalize over this unknown anisotropy when fitting the data. It is interesting to note that the cosmic telescope Gaia [33] is measuring the 3D velocities, see an example in Ref. [34]. This will significantly improve the mass measurements for many objects, including the Milky Way, its dwarfs spheroidal satellites and other objects from local volume.

Dwarf galaxies are small galaxies with masses from $10^{7} M_{\odot}$ to $10^{10} M_{\odot}$. There are two types of dwarf galaxies: those that gravitationaly bound to a bigger galaxy (satellites) and galaxies, that are not bound to any larger halo (field galaxies). An example of a dwarf galaxy is shown in Fig. 2.3. Dwarf galaxies are extremely Dark Matter dominated among all known astrophysical objects with the largest mass-tolight $(\mathrm{M} / \mathrm{L}))_{\odot}$ ratios that can be 100 and larger (e.g. [35, 36] and references therein). It is known that the best mass measurement is at the half-light radius, see the discussion in Section 3.3.1. These measurements show that the stellar mass is not enough to explain the total mass.

- Temperature of gas in galaxy clusters and elliptical galaxies: A cluster consists of hundreds of galaxies that look more or less like point sources inside the cluster 


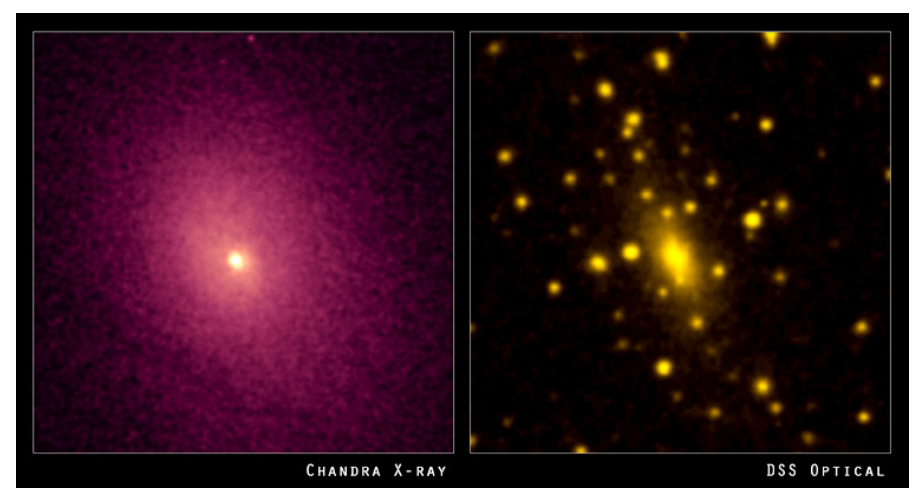

Figure 2.4: The distribution of X-ray gas (left) and galaxies (right) in the cluster of galaxies Abell2029. Credit: DSS.

(see right panel of Fig. 2.4). X-ray observations (see left panel of Fig 2.4) show that the intergalactic medium inside a cluster is filled with a diffuse source of thermal $\mathrm{X}$-ray emission with temperatures in the range of 1-10 keV. Modeling shows that the mass of the gas is $\sim 15$ times larger than the mass of the member galaxies (e.g. [37] and references therein).

In clusters one can therefore apply several methods of mass measurements: reconstruct mass (with some uncertainty) from the motion of galaxies; using the temperature of the X-ray emitting gas; using weak and sometimes also strong gravitational lensing.

The most common method for galaxy clusters is based on X-ray observations. X-ray surface brightness and spectrum allow reconstructing the gas temperature. The average temperature (i.e. the average kinetic energy) is roughly related to the potential energy, i.e. the total mass. As the mean free path of the gas particles is much smaller than the size of the cluster, the thermal equilibrium and the temperature of the gas are local. We can measure the temperature profile $T(r)$ and use it for more detailed mass modeling reproducing $M(r)$ by solving the hydrostatic equilibrium equation

$$
\frac{d p}{d r}=n_{\mathrm{gas}}(r) \frac{d T(r)}{d r}+T(r) \frac{d n_{\mathrm{gas}}(r)}{d r}=-\frac{G M(r) n_{\mathrm{gas}}(r)}{r^{2}} .
$$

Mass measurements in clusters reveal the same picture: only $1 \%$ of the total mass is given by galaxies, $15 \%$ by X-ray gas and $84 \%$ by Dark Matter, see e.g. [38] and references therein. At large enough distances from the center clusters are very much DM dominated. It was observed long ago [39] that the ratio between DM density and the density of normal matter in clusters is very close the the average value of this ratio in the whole Universe (see below).

- Gravitational lensing: Masses of astronomical objects can be measured with weak 


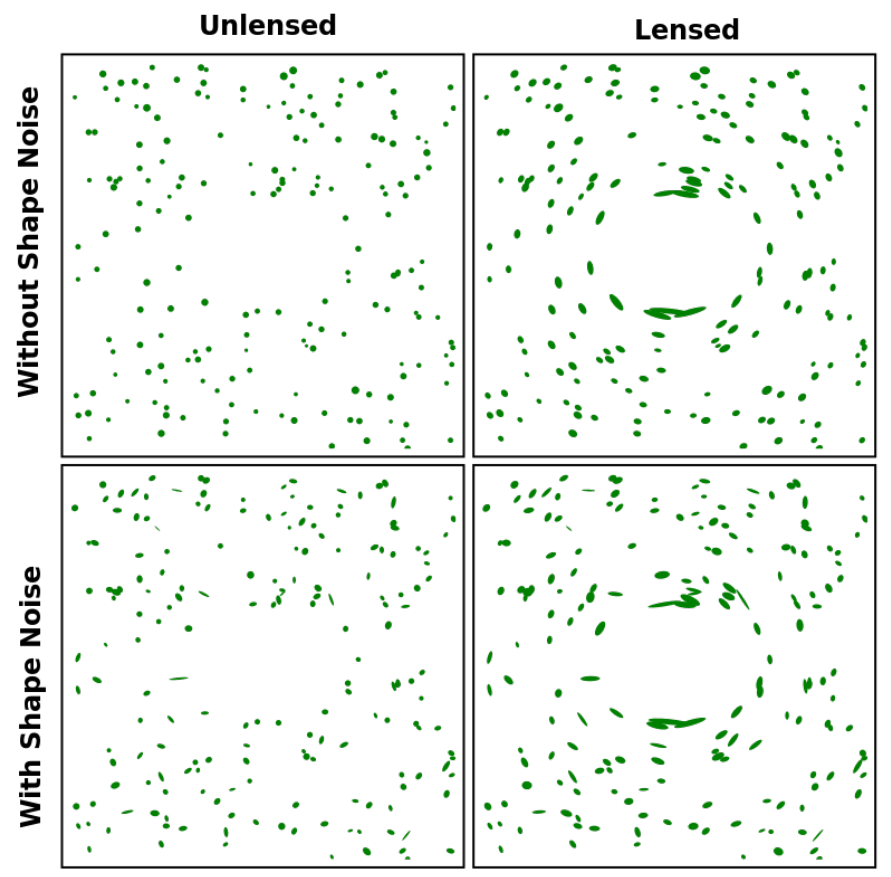

Figure 2.5: An illustration of the effect of weak gravitational lensing. Credit: TallJimbo [CC BY-SA 3.0].

gravitational lensing, see an example in Fig. 2.5. Massive objects act as lenses that distort the light that comes from distant objects, see [40] for a review. This results in overall shear and magnification of the lensed objects. Measuring an average shear one can reconstruct the projected mass of the lens. Applying this method to galaxies and clusters we see once again that the baryonic mass is not enough to explain the lensing effects.

Summarizing these sets of different astronomical observations, we can conclude that astrophysical objects at all scales (from dwarf galaxies with masses from $10^{8} M_{\odot}$ to huge clusters of galaxies with masses $\sim 10^{15} M_{\odot}$ ) indicate the presence of much dark mass that dominates over the mass of normal luminous matter by factors from several to hundreds. These results are obtained using 3 observationally independent techniques.

The analysis of the evolution of the whole Universe at large provides other, cosmological evidence for the existence of Dark Matter and allows to measure it average density.

- Structure formation: The Cosmic microwave background (CMB) is the earliest light that we detect from our Universe. The CMB is isotropic to a very high precision $\left(\delta T / T \sim 10^{-5}[41]\right)$. Together with the fact that the number of photons in the 
Universe is many orders of magnitude larger than the number of protons and electrons, this means that until recombination, when matter was very tightly coupled with light, matter was also homogeneous at the same level as light, $\delta \rho / \rho \sim 10^{-5}$. If there were only Standard Model (or baryonic) matter in the Universe, the overdensities could begin to grow only after recombination, when CMB photons decoupled from charged particles.

As long as $\delta \rho / \rho<1$, the overdensities of a decoupled, non-relativistic component grow linearly with the scale factor in the matter-dominated epoch, so we would naively expect today to have

$$
\delta \rho / \rho=10^{-5} \cdot\left(\frac{1+z_{\mathrm{CMB}}}{1+z_{0}}\right) \approx 10^{-2} \quad z_{\mathrm{CMB}} \approx 10^{3}
$$

which means that there would be no structures today. This is clearly not what we see today. To solve the problem, a matter component that does not interact with light is needed. This substance, Dark Matter, could start clustering much before recombination (it would grow with a scale factor logarithmically in the radiation dominated epoch and linearly in the matter dominated epoch) and prepare wellformed gravitational wells for ordinary matter to fall in.

- Anisotropies of the Cosmic Microwave Background: While approximate (to very high precision) isotropy of the CMB provides strong evidence of the existence of Dark Matter. The spectrum of its tiny anisotropies contains a lot of information about the evolution of the Early Universe and allows to measure the abundance of Dark Matter with impressively high precision [41]. This spectrum is very sensitive to the cosmological parameters and, in particular, to the densities of various components of matter and radiation. Namely, the position of several observed peaks in the CMB spectrum is defined by the total amount of matter in the Universe and the ratio between odd and even picks gives information about the amount of baryonic component. The latest results from Planck show that the abundance of baryonic matter should be more than 5 times smaller [41] than the total amount of matter. The unknown component we associate with Dark Matter. According to Planck, the abundance of DM is $\Omega_{\mathrm{DM}} h^{2}=0.120 \pm 0.001$.

In summary, DM is necessary for our understanding of the formation and evolution of the Universe as a whole as well as the dynamics of various galaxies and clusters (see e.g. $[42-45])$. 


\subsection{The Nature of Dark Matter}

Having so strong and independent evidence that DM exists, what can we say about its nature?

If DM is made of particles, this particle should be massive and electrically neutral. Also, the DM particle should be stable or have a cosmologically long lifetime. Do we have any such a candidate in the SM? The only possible candidate is the neutrino. However, as we discuss below, this particle can contribute at most a few percents of the total DM density.

Neutrino DM. There are two arguments ruling out $\nu$ as DM candidate:

\section{- Tremaine-Gunn bound [46-50]}

If the DM particle is a fermion, there exists a very robust lower bound on its mass. Indeed, let us consider a DM dominated object, for example, a dwarf spheroidal satellite of the Milky Way. With all possible astronomical uncertainties, one can put an upper bound on the size of this object, a lower bound on its mass and an upper bound on the velocity of the particles that are gravitationally bound inside this halo. Combined together, this allows putting a lower bound on the phase-space density. On the other hand, the phasespace number density should not exceed that of the completely degenerate Fermi gas, the maximal phase-space number density given by the Pauli exclusion principle. This means that there exists a smallest mass that is possible for any fermionic DM particle:

$$
\frac{M}{\frac{4 \pi}{3} r^{3}} \frac{1}{\frac{4 \pi}{3} v^{3}} \leq \frac{2 m_{\mathrm{DM}}^{4}}{(2 \pi \hbar)^{3}} .
$$

Let us apply this bound to a so-called classical Dwarf Spheroidal Galaxies (dSphs) of the Milky Way, where velocities of many stars are measured. For example, for Sculptor dwarf galaxy [36] we can take as a proxy of the object size its half-light-radius $r_{h}=283 \mathrm{pc}$, the mass inside this radius $M_{h}=1.4 \cdot 10^{7} M_{\odot}$ and as a characteristic velocity we take the velocity dispersion $v=\sigma_{v}=9.2 \mathrm{~km} / \mathrm{s}$. Substituting these values into Eq. (2.6) we get $m_{\mathrm{DM}}>460 \mathrm{eV}$. Other $\mathrm{dSphs}$ give similar constraints.

Of course, this simple estimate can be made more precise in many aspects. One could discuss the uncertainties in the parameters of dSphs, take into account a more detailed description of the self-gravitating Fermi gas (see e.g. [51]). However, the dependence of the mass lower bound on the actual value of phase-space density is rather weak as $m_{\mathrm{DM}}$ enters in the fourth power in Eq. (2.6). Therefore, one can say that

$$
m_{\mathrm{DM}} \gtrsim 300-400 \mathrm{eV} .
$$


Even if this bound is relaxed to $100 \mathrm{eV}$, as some papers claim (see e.g. [51]), it still would be in drastic contradiction to the cosmological requirement on neutrinos masses if neutrinos would make up all of Dark Matter.

Indeed, interactions of neutrinos are well studied and, therefore, the number density of the SM relic neutrinos can be predicted also quite robustly. In the Early Universe, for temperatures above $T_{\nu} \simeq 1 \mathrm{MeV}$, neutrinos remain in thermal equilibrium with the SM particles due to weak interactions. Below $T_{\nu}$ the rate of weak reactions drops below the expansion rate of the Universe and the number density of neutrinos does not change anymore in the co-moving volume. This allows estimating the number density of one relic neutrino species today,

$$
n_{\nu, 0} \sim T_{\nu}^{3}\left(t_{0}\right) \simeq 112 \mathrm{~cm}^{-3},
$$

with $T_{\nu}\left(t_{0}\right) \approx 1.95 \mathrm{~K}$. The mass density is therefore

$$
\Omega_{\nu \mathrm{DM}} h^{2}=\frac{1}{\rho_{c, 100}} \sum m_{\nu} n_{\nu, 0}=\frac{\sum m_{\nu} \mathrm{eV}}{94 \mathrm{eV}}
$$

where $\rho_{c, 100}=\frac{3 H_{100}^{2}}{8 \pi G}$ with $H_{100}=100 \mathrm{~km} / \mathrm{s} /$ Mpc. Using DM abundance $\Omega_{\mathrm{DM}} h^{2}=0.12$ (Planck 2018 [41]) we conclude that the SM neutrinos could constitute 100\% of DM only if the sum of their masses was

$$
\sum m_{\nu} \simeq 11 \mathrm{eV}
$$

A larger value would overclose the Universe and be ruled out, a smaller value means that there exists another component of Dark Matter.

We conclude that cosmological and astrophysical requirements for neutrinos DM contradict each other and therefore neutrinos cannot be the dominant component of DM.

\section{- Top-down structure formation}

There exists yet another, independent argument, excluding the Standard Model neutrino as a dominant DM candidate. In short, as first shown by [52] in a Universe with the SM neutrino Dark Matter structure formation would happen in the top-down direction, i.e. large objects, like clusters of galaxies, would form before the smaller objects, e.g. galaxies. This happens because neutrinos have velocities close to the speed of light at the beginning of the matter dominated epoch that prevents the growth of small structures and only large structures can be formed. This directly contradicts observations, as at the largest accessible redshifts some galaxies have already formed and clusters have not [38]. 
As the Standard Model neutrino cannot fully explain the nature of Dark Matter, we can say that DM is a Beyond the Standard Model phenomenon.

Many efforts in unraveling the mystery of DM were made in recent decades. However, we still know very little about what is DM. There are at least three main possibilities:

- Is it a new fundamental particle? This is one of the most widely studied hypotheses and we will discuss it in more detail below.

- Can DM be made of Massive Astrophysical Compact Halo Objects (MACHOs)? MACHOs as dominant DM candidates are strongly constrained by e.g. microlensing data $[53,54]$. Another potential problem is that we need to explain where these compact objects come from - we need really a large amount of MACHOs to explain all DM. For macroscopic compact objects, it may not be a simple task to propose a creation mechanism. Also, for example, we do not know how to explain the existence of planets or neutron stars at the time of the CMB decoupling.

Nevertheless, such candidates are considered and perhaps the most interesting one is Primordial Black Holes (PBHs) [55]. PBHs can be created during inflation in a class of inflation models [56]. In these models PBHs have a distribution of masses and can partially escape the current constraints on MACHOs. However, there are many other ways to constrain PBHs - various versions of micro-, femto-, milli-lensing of sources of different nature; interaction of PBHs with neutron stars or white-dwarfs; stability of star clusters; constraints on $\gamma$-rays from the evaporation of small-mass PBHs, etc (for a recent review see [57]). Essentially, there are only two mass windows still open for PBHs Dark Matter - relatively small masses $\geq 10^{15} \mathrm{~g}$ and another in a mass range compatible with LIGO observation of gravitational waves from a binary black hole system. A recent paper [58] argues that the latter window is disfavoured (see also [59] for discussion). Ongoing and future micro-lensing surveys will be able to probe the remaining parameter space of $\mathrm{PBHs}$ relatively soon.

- Another alternative explanation of the DM phenomena in astrophysical objects could be Modified Gravity or Modified Newtonian dynamics (MOND).

This potential explanation has even more difficulties and is considered by many research as excluded (at least as the only explanation of DM phenomena). This class of models, often successful in describing galactic rotation curves [60], start to have difficulties already when the clusters of galaxies are included in the analysis. Even more, problems appear when one includes in the analysis of merging systems, like the famous Bullet cluster [61]. MOND and modified gravity models also can 
not provide a clear picture for the cosmological evidence for DM based on structure formation. Most probably, even if MOND or modified gravity theories do describe correctly some physics of gravitationally bound systems, they still require some new particle to explain all the Dark Matter phenomenology.

Below we will assume therefore that DM is made of some new particles.

\subsection{Properties of Dark Matter particles}

There are many candidates for DM particles in the literature. The masses of these hypothetical particles differ by many orders of magnitude, they have different spins and other transformation properties, they can be stable or have finite (but cosmologically long) lifetime. Leaving sociological bias aside, we can say the particle physics properties of DM are largely unconstrained, see Fig. 2.6. Below we will briefly review some of the suggested candidates. But let us first try to classify possible DM particles in several different model-independent ways:

- According to their primordial velocities: cold, warm and hot Dark Matter.

- Stable or decaying.

- Completely ballistic or self-interacting.

Cold and Warm Dark Matter and their observational difference. An important property of DM particles is their primordial velocities. If DM particles are created nonrelativistic we call them Cold Dark Matter (CDM). These particles can be confined even by very small-sized over-densities and form haloes of all sizes down to a very small cut-off.

Warm Dark Matter (WDM) and Hot Dark Matter (HDM) are particles that are created relativistic. They cannot be confined by an over-density as long as they remain relativistic and their velocities are still close to the speed of light. Momenta get smaller, because of the expansion of the Universe $p \propto 1 / a$. DM particles become non-relativistic and start to be gravitationally bound by the attraction of over-densities (future haloes).

We can divide all DM candidates into three different groups:

- particles that were created non-relativistic (CDM);

- particles that were created relativistic but became non-relativistic before the matter dominated epoch (WDM); 


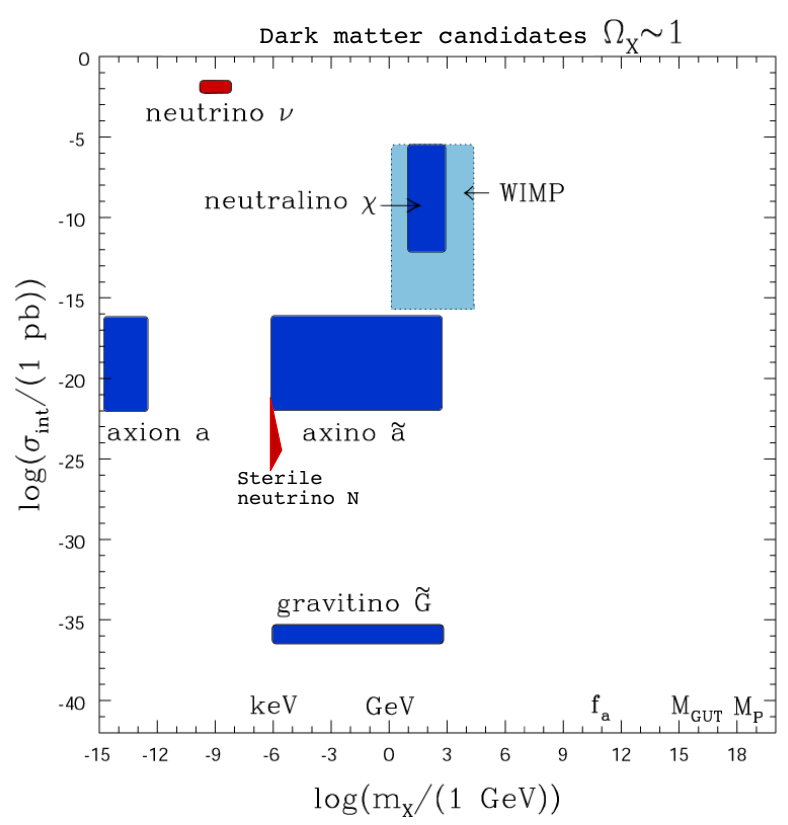

Figure 2.6: Interaction cross-sections and masses of various DM candidates discussed in the literature. Figure is adapted from [62].
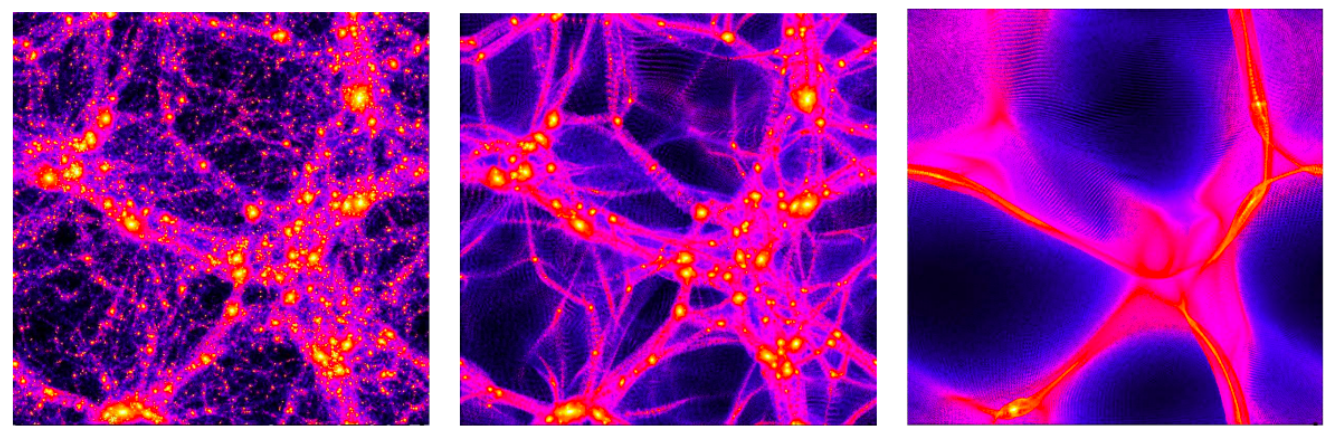

Figure 2.7: Simulation of the large scale structure for the cases of CDM, WDM and HDM (from left to right) cosmologies [63].

- particles that stay relativistic during matter-dominated epoch (HDM).

From the structure formation point of view, the difference between these 3 types of DM is vivid. The distance that a particle travels from the place where it was created to the place where it was gravitationally bound is called free streaming length. Any overdensity that is smaller than the free streaming length is washed out, as particles have random initial velocities, they move chaotically, overdensities and under-densities mix and the distribution becomes homogeneous at the scales equal to the free streaming length and 
below. Larger overdensities do not feel this. The resulting picture is presented in Fig. 2.7, i.e. CDM forms structures of almost any size, WDM washes out small structures and HDM creates only large structures.

The model of cold, collisionless Dark Matter has been so far very successful in describing the observed Universe at large scales: it is fully consistent with the CMB, the distribution of galaxies, the properties of galaxies clusters and filaments [64, 65]. However, Cold and Warm DM are indistinguishable at large scales and therefore are both allowed and equally good. The difference between them appears only at small scales.

In CDM there are clumps of DM of all sizes (down to some small-scale cut-off much below one solar mass [66]), galaxies like the Milky Way have more and more substructures of smaller and smaller sizes. In WDM the number of structures that are smaller than the free streaming length quickly drops to zero. The main difficulty is that small haloes may be very difficult to observe. Indeed, for a halo to be visible now it is important that at the early stage of the halo evolution it confines gas and eventually stars are formed. At the same time, if primordial hydrogen is already hot at the time of halo formation, it can fall into a halo only if this halo is massive enough. Primordial hydrogen is heated during reionization by the light of first stars. We do not know exactly to what temperature it is heated. But in simulations, this temperature can be large enough such that we can expect that small haloes cannot confine the gas and today they are made only of DM.

However, these relatively small, DM-only haloes can only be observed via gravitational lensing. Currently there are two methods to use strong lensing for detecting or excluding such small DM-only haloes: perturbations in Einstein rings [67] which should be perfectly circular in absence of small haloes, and flux ratios of multiply imaged sources [68-70], which should be order-1 ratios in absence of small haloes. The new cosmic telescope Euclid (which will be launched in 2021) will be able to observe enough gravitational lenses for proving or excluding the existence of small DM-only haloes, and either exclude CDM or very severely constrain WDM [71, 72].

In the literature the so called "small-scale problems" of CDM are often discussed (for a review see [73]).

- I. Fewer dwarf galaxies are observed in the Milky Way and M31 than CDM predicts. For smaller haloes, the discrepancy is large between observations and CDM simulations (missing satellites problem) [74-76].

- II. Let us define the slope of the DM density distribution as $\rho \sim r^{-\gamma}$ in the central part of haloes. Pure CDM simulations predict $\gamma$ close to 1 (cusps), but in many objects it is claimed $\gamma<1$ describes observational data much better (cores) (corecusp problem) [77, 78].

- III. Over-prediction of large satellites. The Galaxy has only 3 satellites with the maximum-circular velocity $V_{\max }>30 \mathrm{~km} / \mathrm{s}$. But naively CDM predicts $\sim 10$ sub- 
haloes with $V_{\max }>30 \mathrm{~km} / \mathrm{s}$ for a Milky Way size galaxy. These objects are too large to say that they have no stars in them. Why we do not see these 10 sub-haloes? (too-big-to-fail problem) [79, 80].

Some other potential discrepancies between the data and the predictions of $\Lambda \mathrm{CDM}$ model are also discussed in the literature, including e.g. the underabundance of the field dwarf galaxies or the diversity problem (see e.g. [81, 82] or [83] for a review and references therein).

All these phenomena can, however, be accommodated in CDM if one takes into account all possible observational uncertainties, variance in the initial conditions or baryonic effects [82, 84-87]. Therefore, to choose between CDM and WDM models observationally we need to look at other observables, like Lyman- $\alpha$ forest $[88,89]$ or gravitational lensing $[67,90]$ (see the discussion above).

Stable, decaying or annihilating DM. DM particles should be stable or have a lifetime longer than the age of the Universe. An important part of a DM model is a production mechanism - we have to specify in what way this particle is produced in the Early Universe and check that it has the correct abundance to account for all of the observed Dark Matter. Of course, DM particles can be produced from some other new particles or fields, for example, they can be produced via direct interaction with an inflaton field. However, in a somewhat more minimalistic scenario, DM particles are produced from interactions with the Standard Model matter. In this case, the same interactions can be responsible for DM annihilation or decay.

If a DM particle decays or annihilates into SM particles, we can in principle detect a signal from these processes. If the DM particle is relatively light (e.g. a fermion with its mass just above the Treiman-Gunn bound, i.e. in the keV range) it can decay only into neutrinos and photons. Such decays often result in a monochromatic line in the spectra of photons, a signal that is relatively easy to identify. Heavier particles can decay also into charged SM particles producing a more complicated signature in the spectra of photons of various energies and in cosmic rays. Even if the lifetime is very long or annihilation cross-section is small, the amount the Dark Matter is so large that we can expect a very significant signal from such processes.

For example, in the case of decaying Dark Matter non-observation of such signal puts a bound on the DM lifetime to be orders of magnitude longer than the age of the Universe. We will discuss the difference between the cases of decaying and annihilating DM and corresponding detection strategies in Section 2.5.

DM self-interactions. Many observations show that any interactions of DM with the Standard Model particles are very strongly constrained (see e.g. [91, 92]). At the same time, the constraints on the cross-section of the DM self-interaction are many orders of 
magnitude less stringent. This cross-section can be as large as the strong interaction between nucleons (for a recent review, see [83]).

There are many reasons that make SIDM an interesting and well-motivated scenario.

- For astrophysics, SIDM is interesting as it changes the picture of structure formation at small scales (as compared to CDM) [93]. It was pointed out in the literature [94$101]$ that SIDM could provide a resolution of the above-mentioned small-scale problems of $\Lambda$ CDM. ${ }^{1}$

- For particle physics SIDM models have a very interesting phenomenology that allows bringing together the data from cosmology, astrophysics, accelerators, indirect and direct DM searches (see below). Of course, any strong indication of the existence of DM self-interaction would give invaluable input for the development of the particle physics beyond its Standard Model by suggesting a preferred class of models. Cosmological and astrophysical data would then provide unique information for particle physics (see e.g. [104-106]).

Constraining the cross-section of SIDM from astronomical data is very important. Recently a lot of work has been done in this direction (see e.g. [94, 95, 98-100, 103, 107-122] or see [83] for a review). For this reason SIDM is now supported by the public numerical code DarkSUSY [123].

There are two types of questions that can be addressed here. First, we would like to know what kind of upper bound on DM self-interaction can be derived from the data. Second, we can ask ourselves, what self-interaction cross-section is required to explain, for example, the sizes of the observed cores? We can ask these questions assuming that the cores are caused by the properties of the DM particle, even if there could be other possible mechanisms to explain DM density cores and other small-scale challenges of CDM (see the discussion above).

According to the current opinion in the literature, it is probably required to have DM self-interaction cross-section $\sigma / m \gtrsim 0.1 \mathrm{~cm}^{2} / \mathrm{g}$ to produce potentially observable cores in the central parts of DM dominated objects (see e.g. [73, 83, 97, 105, 124-126]). The upper bounds on the cross-section are reported to be around $1-2 \mathrm{~cm}^{2} / \mathrm{g}$, see the detailed discussion in Section 3. These constraints are obtained from observations of merging systems, including the Bullet cluster, as well as ellipticities of the galaxies (see e.g. [96, 127-130]).

\footnotetext{
${ }^{1}$ For SIDM to suppress small satellites a scattering with a dark radiation component is needed. Such a component appears rather naturally only in models where the self-interaction is mediated by a light particle $[102,103]$.
} 
The challenges in constraining SIDM astrophysically are related to: (i) observational uncertainties and (ii) difficulties in modeling of baryonic effects [105, 131, 132]. Additionally, properties of the real observed haloes and, in particular, the sizes of the cores have significant scatter. This variability is caused by the baryonic content, which is different in different objects and by individual initial conditions and histories of the haloes (see e.g. [133]). To constrain DM self-interaction cross-section and reduce systematic uncertainties, we need therefore to base our analysis not on individual objects, but find a way to use the whole ensemble of the available observed objects.

In paper I [28] we have introduced an efficient and robust method that implements such a strategy. This method is discussed in detail in Section 3.2 and further developed in Section 3.3. In paper II [29] we have systematically checked semi-analytic models of SIDM haloes that are discussed in the literature $[83,105,134]$ by comparing them with numerical simulations (see the detailed discussion in Section 3.2). We have demonstrated that the agreement of the model with the simulations is not sufficient to make it possible to obtain robust observational constraints on DM self-interaction cross-section using this model. We conclude that a more direct comparison between observations and simulations is necessary (see Section 3.3 for discussion).

\subsection{Examples of models for DM particles}

A multitude of DM models have been proposed. Properties of DM candidates in these models are very different as well as physics behind them. Here we discuss three very different particle DM models. These models are representative of their classes and many variations of these particular models could be considered within each class (for more details see e.g. [38, 135, 136] and references therein).

The first candidate class is Weakly Interacting Massive Particle (WIMP), which has been proposed in the seminal paper by Lee and Weinberg [137]. WIMPs are stable, but they could annihilate into SM particles. They are produced thermally in the Early Universe. Once the annihilation rate becomes lower than the Universe expansion rate, such particles are effectively decoupled from the SM plasma and their concentration is 'frozen'. WIMPs are massive particles with masses from a tenth of $\mathrm{GeV}$ to hundreds of $\mathrm{TeV}$. This is an example of CDM. Quite amazingly, the value of the annihilation cross-section required for providing the observed DM density is close to that of the weak cross-section. This fact, known as "WIMP miracle", is the main driving force of the direct DM detection program. Indeed, the same interaction that allows producing WIMPs in the Early Universe could be responsible for their scattering with a nucleus (see the discussion of the direct detection experiments below).

WIMPs can also be self-interacting e.g. if the dark sector contains not only one single DM particle but also a mediator of the interaction between DM particles, see e.g. [83] for 
a review. As it was discussed, SIDM could have an interesting impact on astrophysics and cosmology. Its particle physics phenomenology depends on the spin of the mediator, the mass ratio between the DM particle and the mediator, etc. One simple (but viable) SIDM model and its searches at accelerators, direct detection as well as their combination with astrophysical constraints are discussed in Section 5.

Another candidate is the so-called sterile (right-handed) neutrino, see e.g. $[17,138$, 139]. This is an example of Warm DM. Sterile neutrinos naturally arise once one tries to explain neutrino masses via the so-called see-saw mechanism. ${ }^{2}$ This mechanism implies a mixing between active and sterile neutrinos. One consequence of such mixing is that sterile neutrinos can decay into active ones and a photon (radiative decay). Therefore, even though sterile neutrino DM cannot be detected directly, they can be very efficiently searched astrophysically. From the astrophysical constraints on the mixing angle, it is known that DM sterile neutrino interacts so feebly with the SM matter that it is never in thermal equilibrium in the Early Universe. Therefore its primordial velocity spectrum has a non-thermal shape.

The last example is the axion, or, more broadly, the Axion-like particle (ALP), for a review see e.g. [143, 144]. Axions were proposed by Peccei and Quinn [145] to solve the Strong CP problem. In general, it is a very light CDM candidate with the mass $m_{a} \ll 1 \mathrm{eV}$. The axion $a$ interacts with the SM as

$$
\mathcal{L}_{\text {int }}=g_{a} a F_{\mu \nu} \tilde{F}^{\mu \nu}
$$

where $\tilde{F}^{\mu \nu}=\frac{1}{2} \varepsilon^{\mu \nu \sigma \rho} F_{\sigma \rho}$. In the original model, the interaction strength $g_{a}$ was proportional to the axion's mass, but one can consider a more general situation of axion-like particles (ALPs), that does not solve the Strong CP problem but still are viable DM candidates. In ALP models, there is no connection between interaction strength and mass of the particle.

It is also possible to consider axions as an example of the so-called fuzzy DM [146] a very light DM particle with so large quantum wave-length that quantum effects change the inner structure of DM haloes. In all such models the DM particle is extremely light $\left(\sim 10^{-22} \mathrm{eV}\right.$ in the case of fuzzy DM) and the production mechanism - vacuum realignment [147] - is very different from that of cold DM. ALPs are also a subject of a direct experimental search, but, again, the mechanism is very different. 


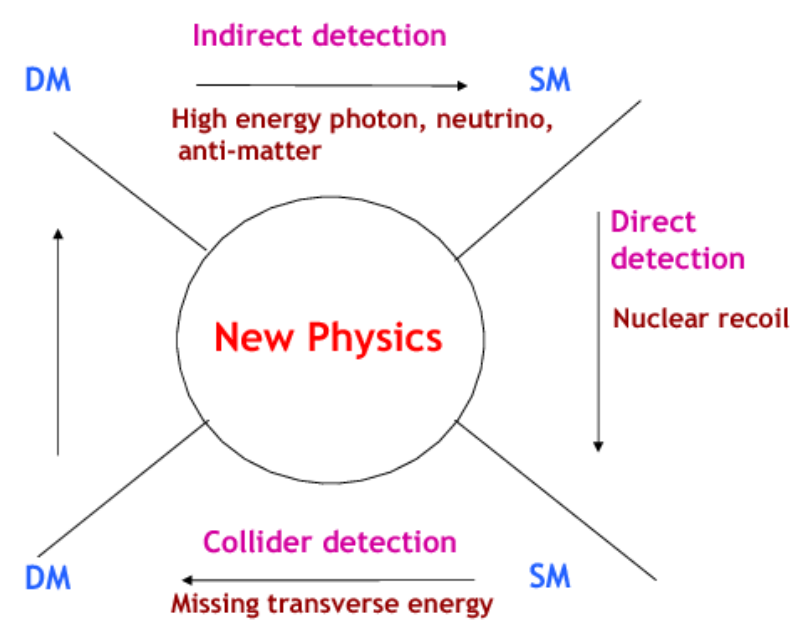

Figure 2.8: Illustration of the possible approaches to DM detection, using WIMPs as an example. Taken from [148]. For other types of Dark Matter candidates some of these approaches, e.g. accelerator searches or direct detection, may not be available.

\subsection{Dark Matter detection}

The possible methods of DM detection are:

- Direct detection: find a signal of the interaction of DM with a nucleus in the laboratory.

- Indirect detection: find a signal from decay/annihilation of DM from space.

- Search at accelerators: find events with DM particles at colliders.

Many other efforts exist to search for signatures of Dark Matter particles or their decay or annihilation via their influence on the CMB, on stellar burning processes [149], in precision measurements[150], etc.

\subsubsection{Direct detection}

The goal of direct detection experiments is to detect in laboratories DM particles from the Milky Way halo around us. For such an experiment we need to know details of the interaction of DM particles with the SM. Therefore, this type of searches should be designed for a concrete type of DM candidates. For this reason, it makes sense to discuss it only for

\footnotetext{
${ }^{2}$ In fact, it is enough to add to the SM two sterile neutrinos to explain the data on neutrino flavor oscillations. These two particles interact with the SM too strongly to be Dark Matter. The third right-handed neutrino can have much more feeble interaction such that it can be a very good DM candidate [140, 141]. Its contribution to neutrino masses is then negligible [142].
} 

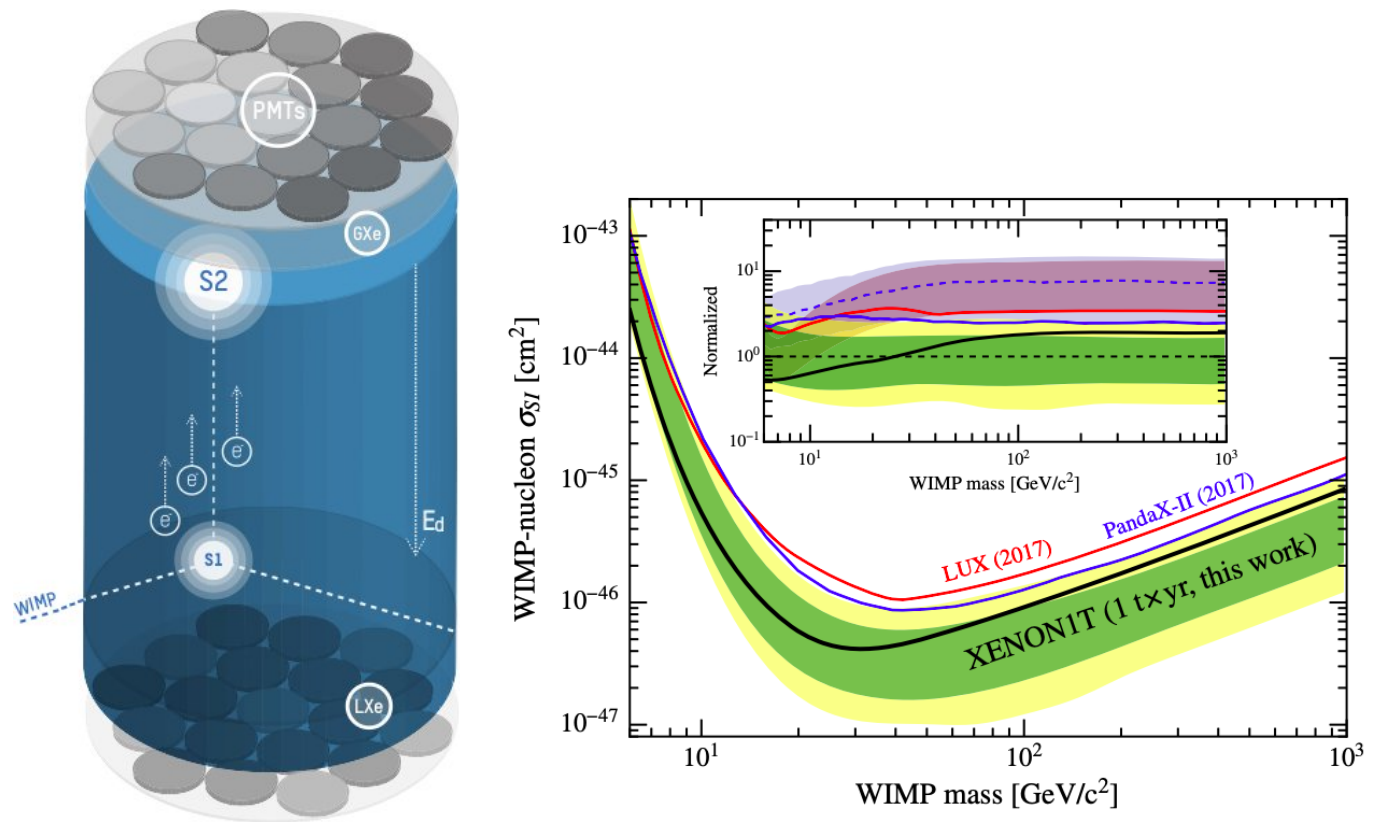

Figure 2.9: Left: Scheme of the operation of the XENON experiment. Credits: Gaudiman [CC BY-SA 3.0]. Right: Upper limits on spin-independent cross-section $\sigma_{S I}$ with $1 \sigma$ (green) and $2 \sigma$ (yellow) sensitivity bands. Taken from [153].

concrete examples. Below we consider two such examples - direct detection of WIMPs and direct detection of axions.

Probably the only question relevant for direct detection that can be discussed modelindependently. What do we know about DM halo properties around Earth? The average $\mathrm{DM}$ density is known to be $\rho_{\odot}=0.4 \mathrm{GeV} / \mathrm{cm}^{3}[151]$ and the average velocity of DM particles $\sim 200 \mathrm{~km} / \mathrm{s}$.

However, even the local DM density may be model-dependent. Indeed, what is relevant for the direct detection is not the average density in the Milky Way at the distance $r_{\odot}$ from the Galactic Center, but the local density that can be encountered by Earth in the process of its movement around the Sun and, together with the Sun, around the Galactic Center. This is already model-dependent. Indeed, in the cold DM (e.g. WIMPs) one could expect small dark clumps, in some cases down to the size of Earth. In the case of axions, the DM distribution may have the so-called caustics, where the density can be very high [152].

Direct detection of WIMPs. As we discussed above, WIMPs can scatter on nucleons and electrons via the same interaction that is responsible for the annihilation and production in the Early Universe (see Fig. 2.8). For spin-independent scattering, the cross-section of the scattering on nucleons is much larger than on electrons [38], as the center of mass, energy is larger. This also means that the materials with a large atomic mass have an 


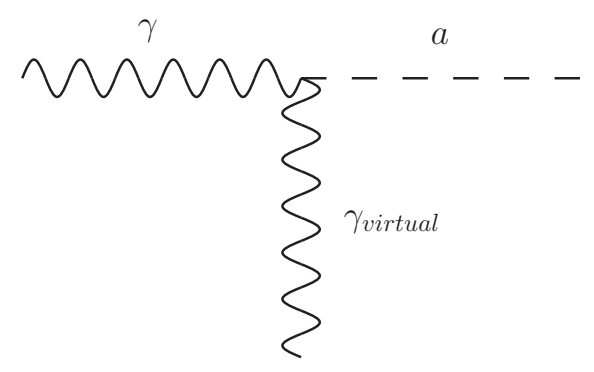

Figure 2.10: Feynman diagram of the Primakoff conversion.

advantage. The typical kinetic energy of a WIMP is

$$
E_{\chi, \text { kin }} \sim \frac{m_{\chi} v^{2}}{2} \sim 0.02 \mathrm{MeV}\left(\frac{m_{\chi}}{100 \mathrm{GeV}}\right)
$$

This means that as a result of such a scattering the nucleon will remain a part of the whole nucleus, but the atom will be ionized. Therefore, potentially we will have three signatures of the scattering: a (relatively) high-energy electron, nucleus and, additionally, a phonon.

There are many experiments that aim for direct detection of WIMPs, e.g. XENON [154], LUX [155], ZEPLIN [156], EDELWEISS [157], CRESST [158, 159] etc. As a characteristic example of such experiments, we can consider the XENON experiment [154] (see Fig. 2.9) located in the Gran Sasso laboratory in the Italian Alps. The detection volume is filled with liquid xenon. A galactic WIMP that scatters on a nucleus ionizes its atom. An ion that propagates in xenon creates $178 \mathrm{~nm}$ ultraviolet photons, that are detected by photo-multipliers PMTs (S1 signal). Electrons in the electric field $E_{d}$ drift to the detectors and can be detected ( $\mathrm{S} 2$ signal). The combination of S1 and S2 signals is sufficient to distinguish the DM signal from the background.

Direct detection of axion-like particle Dark Matter. ALPs can be searched via a specific mechanism called the Primakoff conversion, that converts axions into photons in the external electromagnetic field, see Fig. 2.10. For instance, the ADMX experiment [162] (see Fig. 2.11) uses microwave cavity to increase axion conversion rate in the presence of the background microwave photons in the cavity. ADMX measures the power of converted photons up to the level $P \sim 10^{-26} \mathrm{~W}$ [38].

\subsubsection{Indirect detection}

DM indirect detection also aims to detect Dark Matter particles that exist in a halo, often in the Milky Way halo or one of its satellites. The main difference with direct detection is that the interaction of the DM particle with ordinary matter - decay or annihilation to SM particles - happens not in a laboratory, but directly in space, sometimes very far from Earth. If the products of decay or annihilation are charged particles, it is very challenging 

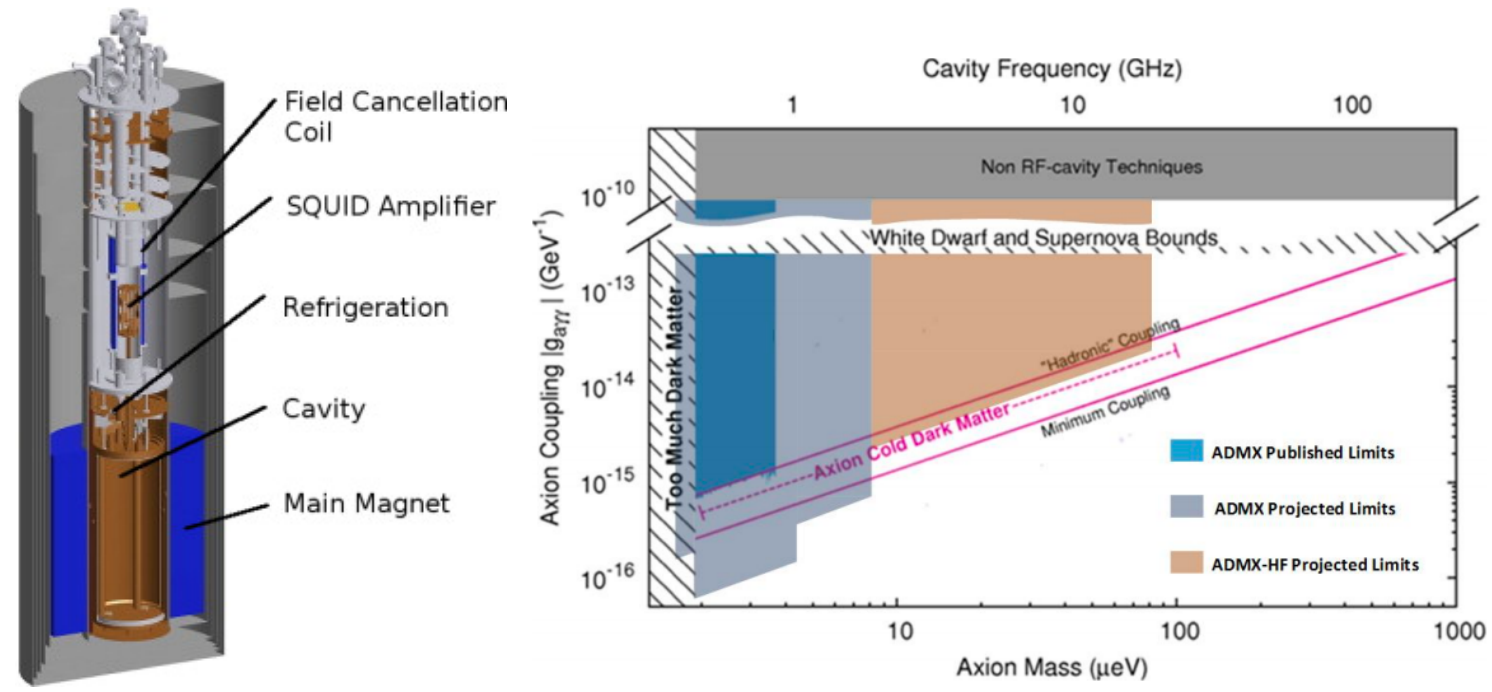

Figure 2.11: Left: Scheme of the ADMX experiment [160]. Right: Sensitivity of the ADMX experiment for the parameter space that already excluded (blue) and that will be explored (green) [161].

to know even the direction towards the source, as charged particles change their direction many times moving in the Galactic magnetic field of the Milky Way that has quite nontrivial structure. In this case, e.g. a feature in the spectrum of anti-particles can be used as a possible signature of DM decay [20]. Below we will discuss only the case when the signal from annihilation (decay) is in photons [163] (so-called "prompt" emission). In the case of photons, the direction towards the source is known. The main challenge, in this case, is to distinguish it from the backgrounds.

What signal one should expect? As we know, DM particles are distributed in galaxies in such a way that in the center of a galaxy there are more DM particles than at the edges. So we expect the strongest signal from the center.

The differential $\gamma$-ray flux, $\Phi_{\gamma}$, for the promt emission integrated over the solid angle $\Delta \Omega$ can be written as [151]

$$
\frac{d \Phi_{\gamma}}{d E_{\gamma}}\left(E_{\gamma}, \Delta \Omega\right)=\Phi^{P P}\left(E_{\gamma}\right) \times J / D(\Delta \Omega),
$$

where $\Phi^{P P}\left(E_{\gamma}\right)$ is proportional to the annihilation cross-section or the decay width and depends on particle physics only, while $J / D(\Delta \Omega)$ (factors for annihilation/decay) takes into account the geometry of the system and the DM density distribution. For the annihilating Dark Matter the $J$ factor depends on $\rho^{2}$, because for the DM annihilation two DM particles should interact. The definition of the $J$ factor is:

$$
J(\Delta \Omega)=\int_{\Delta \Omega} \int \rho^{2}(l, \Omega) d l d \Omega,
$$



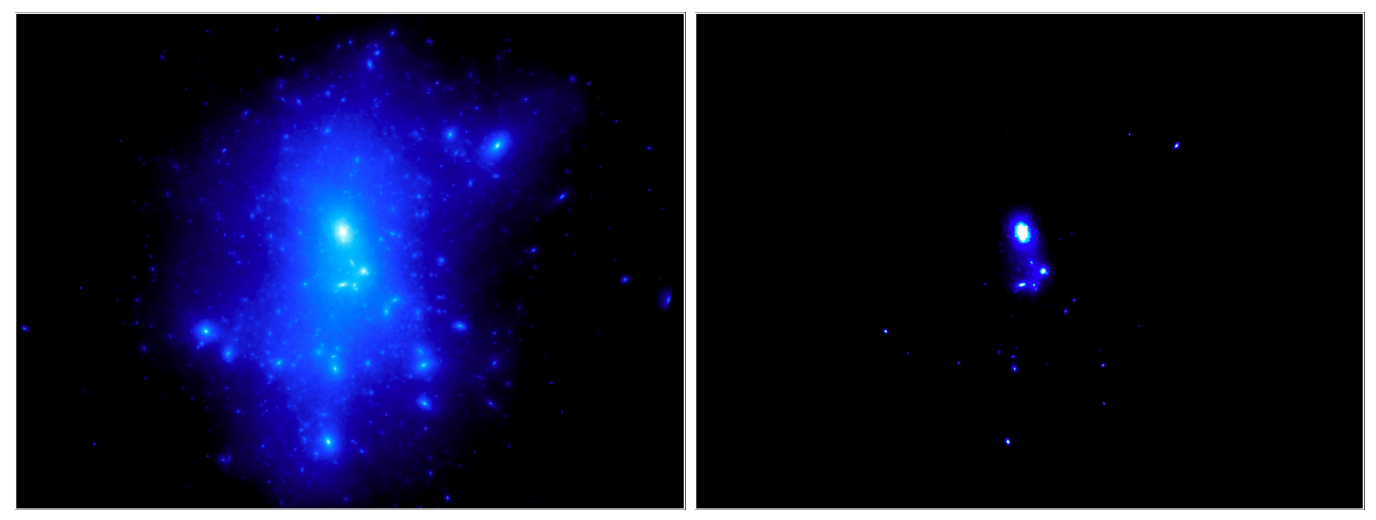

Figure 2.12: Difference between signals from decaying (left panel) and annihilating DM (right panel) calculated for a simulated Milky Way size galaxy. Brightness represents the value of $J$ or $D$ factors, correspondingly. Taken from Subinoy's talk.

where $l$ is the distance along the line of sight and i $\Omega$ is the solid angle. For a decaying particle, only one DM particle is needed to produce a signal, therefore, the $D$-factor is linearly proportional to the density,

$$
D(\Delta \Omega)=\int_{\Delta \Omega} \int \rho(l, \Omega) d l d \Omega .
$$

As a result, the signal from annihilating DM is much more concentrated in the center of a halo, while the signal of decaying DM has a wider distribution, see Fig. 2.12.

Below, we will discuss the case of annihilating DM. Therefore, let us describe the expected signal in more details for this case. We have discussed the spatial morphology of the DM annihilating signal (of course this morphology depends also on the DM density profile in a target, see Section 4 for the detailed discussion for the case of the Milky Way). Also, we need to describe its spectral properties. These are not unique and depend on a particle physics model and available annihilation channels. First of all, we have to distinguish two scenarios of $\gamma$ photons creation. The first one is the so-called prompt emission, i.e. the scenario when photons are produced directly in the annihilation (e.g. DM $+\mathrm{DM} \rightarrow \gamma \gamma$ ) or from a very fast decay of secondary particles (e.g. DM $+\mathrm{DM} \rightarrow \pi_{0}+\cdots \rightarrow \gamma \gamma+\ldots$ ), see a review [163] for more details. In this case, the photons are produced in the same place where the annihilation happens. Another possibility is that there are charged particles (electrons, protons) that are produced in the initial annihilation. Typically, they travel some distance from the creation point and produce photons for example via the Inverse Compton process. We call this secondary photon emission.

Such a $\gamma$-ray signal is more diffuse than the prompt one. Below we assume that the contribution of secondary emission is negligible (see Section 4) and the morphology of the signal is defined by the $J$-factor.

This spectral shape of prompt emission of the signal can also be very different. In the 

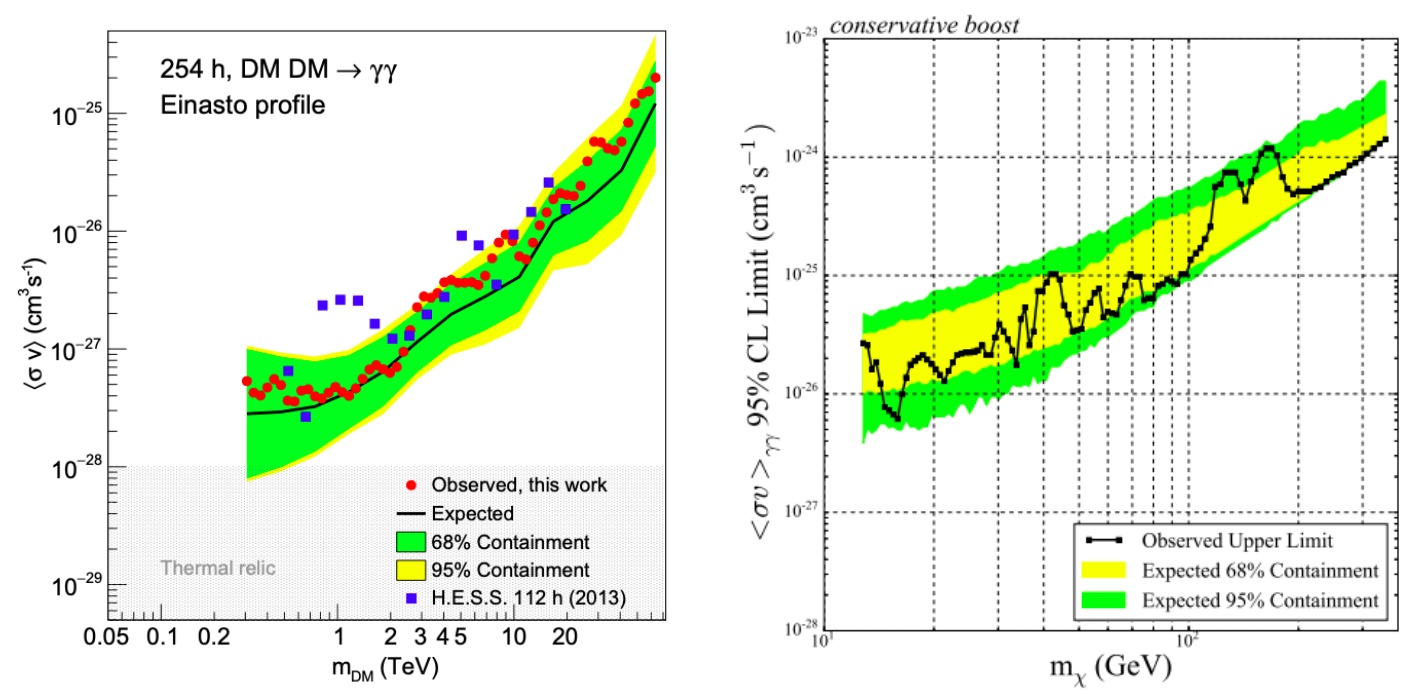

Figure 2.13: The bounds on the cross-section for the case of annihilation into a monochromatic $\gamma$-ray line, obtained by the H.E.S.S. observations of the Galactic Center [164] (left) and Fermi-LAT [165] (right).

simplest case of DM $+\mathrm{DM} \rightarrow \gamma \gamma$ the energy of the final photon is fixed $E_{\gamma}=M_{\mathrm{DM}}$. Such a narrow line signal is considered to be a "smoking gun" signature of DM annihilation (decay) [166]. This case was intensively studied by H.E.S.S., see Fig. 2.13 with bounds on the annihilation cross-section in such a model. Other examples of "smoking gun" signatures are discussed e.g. in $[163,167]$. Let us consider for example the case of DM + $\mathrm{DM} \rightarrow W^{+} W^{-}$annihilation. $W$ boson has pure leptonic and hadronic decay modes, the hadronic branching ratio is $\mathrm{BR}(W \rightarrow$ hadrons $) \approx 66 \%$. In such a case a number of pions are created (average number of charged pions is 15.7 [14], only $\pi^{0}$ are important for prompt emission of photons). This is a many-body decay process, so the spectrum of photons is wide and has the maximum below $M_{\mathrm{DM}}$ (see Fig. 2.14).

Backgrounds. In real life the DM annihilation signal should be of course detected against various backgrounds. The sources of the background are CR and Galactic Diffuse Emission $(G D E)$, which we describe below. The CR background is especially important for the Imaging Atmospheric Cherenkov Telescopes (IACTs) (see a detailed discussion and the description of these instruments in Section 4). The difference in spatial morphology between the signal and the background is illustrated in Fig. 2.15.

The first background, CRs, is isotropic as it is produced by charged particles, which are entangled in the magnetic field of the galaxy and "forget" the direction towards their place of creation, see Fig. 2.16. CRs are composed mainly of protons with a small fraction of helium, iron and other nuclei as well as electrons.

The second background is the diffuse emission from the Galactic disk. This background component consists of two parts - one is the emission from unresolved galactic 


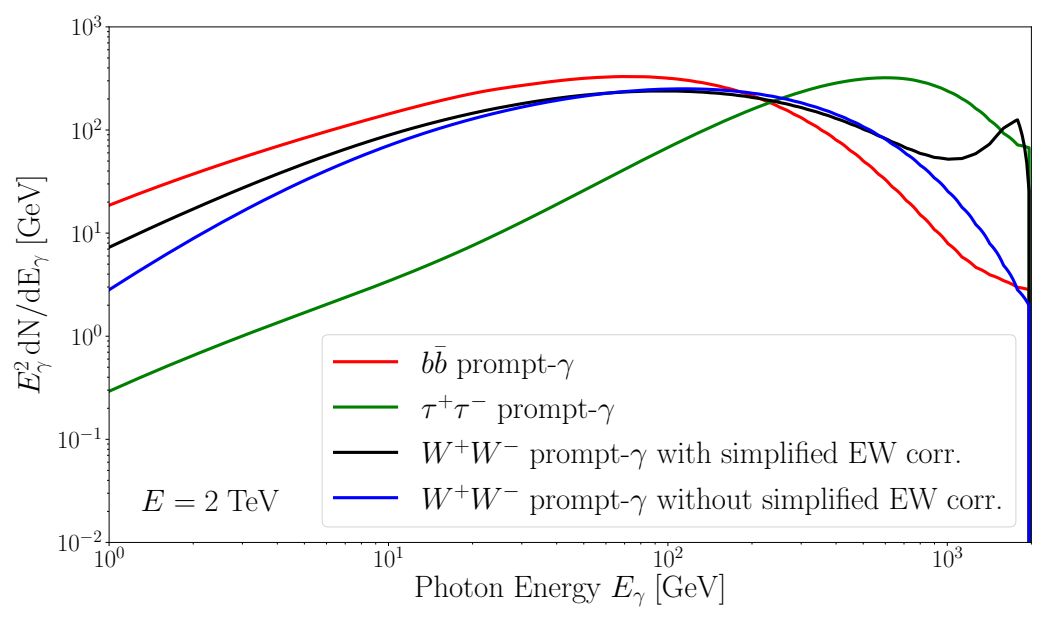

Figure 2.14: The DM spectrum for promt emission for different annihilation channels for $M_{\mathrm{DM}}=2 \mathrm{TeV}$. Taken from paper IV [30].
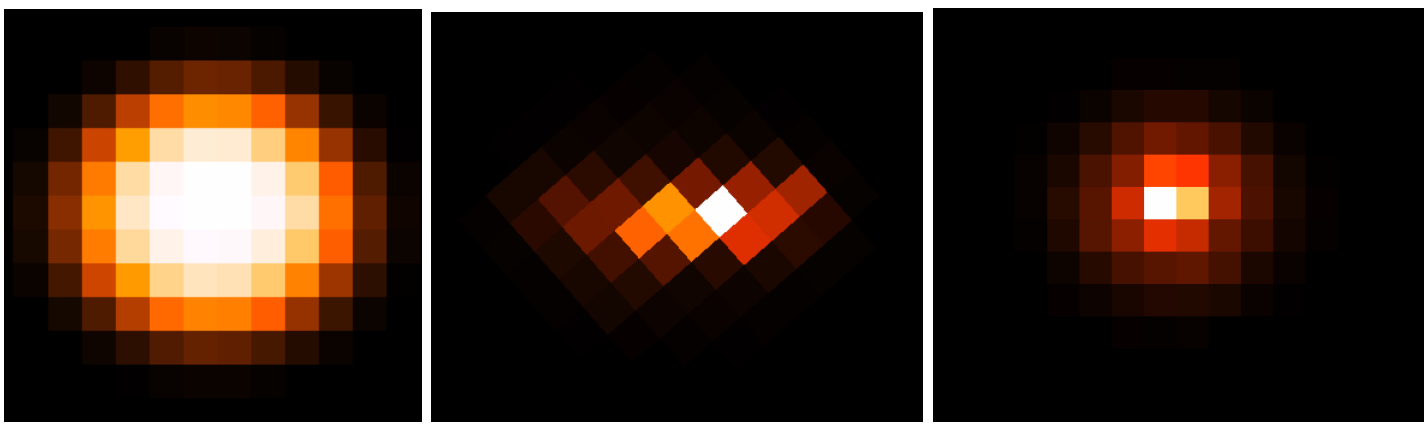

Figure 2.15: The simulated signals for an atmospheric Cherenkov telescope from CRs (left), GDE (middle) and DM (right) (generated with ctools, see Section 4).

point sources and the other one is the "true" Galactic Diffuse Emission.

As for unresolved point sources, for example, a population of galactic millisecond pulsars (MSPs) can significantly contribute to the observed GDE. Close to the Galactic Center region this population is the main candidate for the explanation of the Fermi/LAT "Galactic Center GeV excess" [169]. The parameters of the MSPs distribution that are required to explain these data seem to be consistent with the models of stellar evolution [170]. At the same time, the exact form of this distribution as well as its contribution to the GDE remains quite uncertain.

The true GDE originates from interactions of high-energy CRs with interstellar photons or interstellar gas (mainly $H$ ). There are 2 possible scenarios of producing GDE photons which depend on the primary CR particle:

- a high energy proton with a photon from the background can create $\pi^{0}$ which quickly decays into a pair of photons (this mechanism is important for the high-energy GDE 


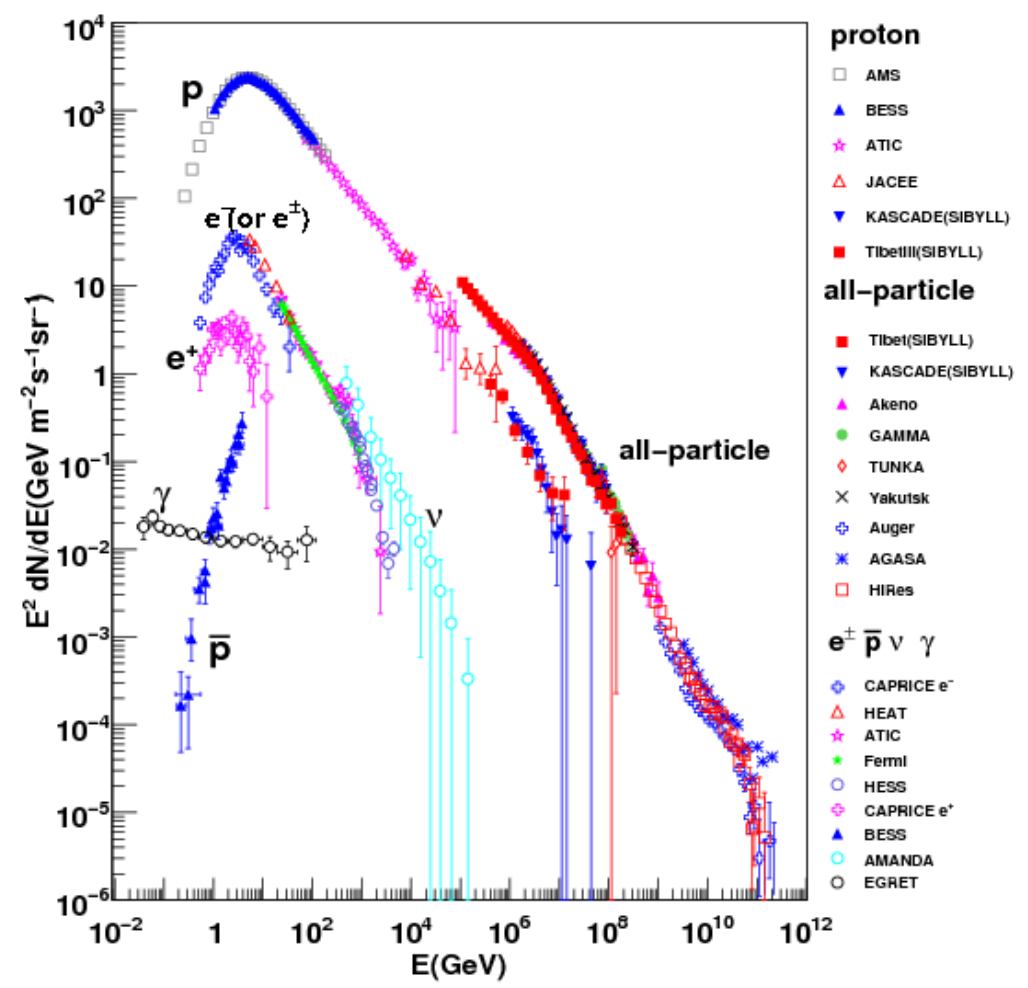

Figure 2.16: The CR spectrum observed by different data. Taken from [168].

background, see Fig. 2.17). Also, protons can be created by Bremsstrahlung;

- high energy electrons can create photons via four different processes: Inverse Compton; Bremsstrahlung; annihilation of positrons and synchrotron radiation.

Inverse Compton is a process in which a high-energy electron transfers its energy to a low-energy photon. In the galaxy, there are two main components of the low-energy photon background: CMB photons and starlight. CMB photons have a constant density, while diffuse galactic radiation is denser in the center of the galaxy.

Bremsstrahlung and annihilation of positrons give a contribution to the photon spectra with energies below $1 \mathrm{GeV}$ and below $1 \mathrm{MeV}$.

To distinguish the DM annihilation signal from CRs and GDE backgrounds we can use both its spatial morphology (see Fig. 2.15) and its spectral properties. Fig. 2.18 shows an example of a spectrum measured by a $\gamma$-ray telescope for the model containing CRs+GDE+DM. 


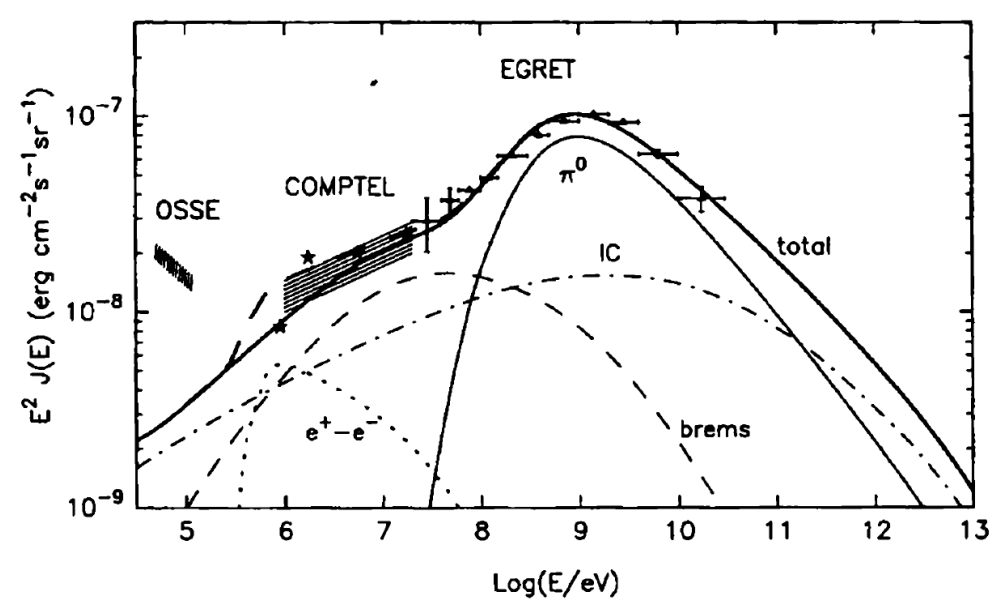

Figure 2.17: The contribution to the total flux of GDE photons produced by electronic and nucleon components [171].

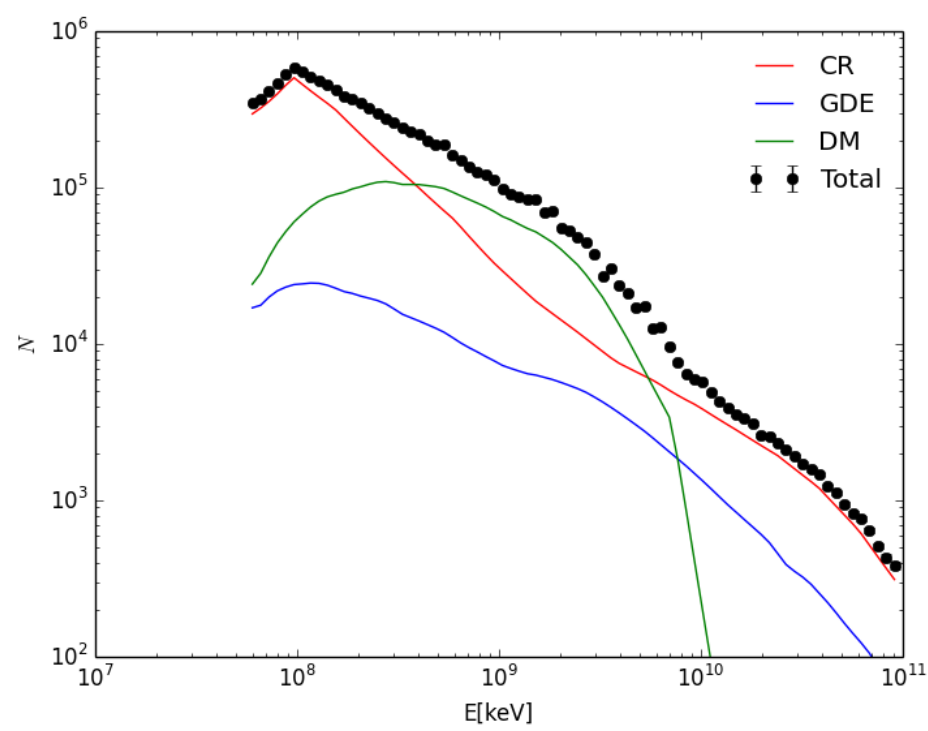

Figure 2.18: An illustration of the contribution to simulated number of events from CRs (red curve), GDE (blue curve) and DM (green curve) with $M_{\mathrm{DM}}=2 \mathrm{TeV}$ for the $W W$ channel. Black points are the total number of events.

\subsubsection{Search at accelerators}

DM, if not stable, should be a very long-lived particle. So if it is produced at any accelerator experiment the expected signatures are missing energy and/or missing momentum. For hadron colliders like the LHC, it is impossible to check the full conservation of energy, as the particles produced with a small angle fly into the pipe and their properties cannot be measured. Instead, the total momentum in the transverse direction $p_{T}$ has to be conserved 


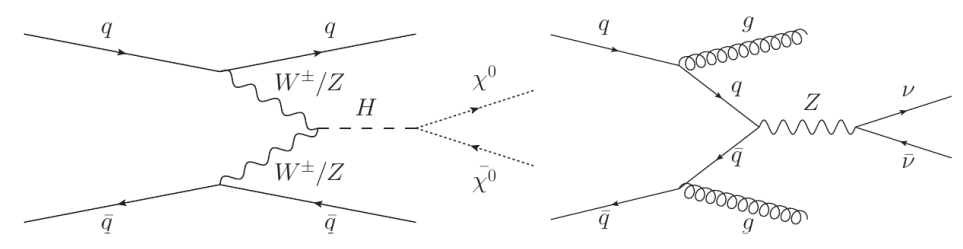

(a) Signal (b) Strongly produced (QCD) $Z+$ jets

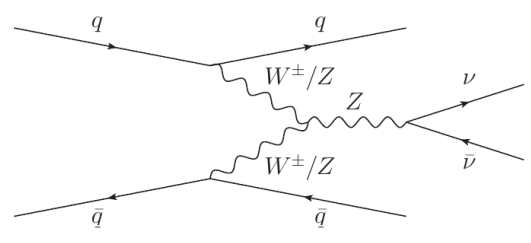

(c) Weakly produced (EW) $Z+$ jets

Figure 2.19: An example of the Higgs production in vector boson fusion and its decay into DM particles (a). Diagrams (b) and (c) show the main SM background. Figure is taken from [172].

and can be measured. Therefore, to search for Dark Matter at the LHC one should search for missing $p_{T}$. The main background for such processes is given by evens with SM neutrinos (see an example in Fig. 2.19). It is possible to reduce such a background assuming a certain model. In such a case one can use model dependent signatures, introducing such cuts on the initial and finale states that reduce significantly the number of SM events and do not affect the signal, e.g. a high-energy photon + missing $p_{T}$, a monojet + missing $p_{T}$, a high-energy lepton + missing $p_{T}$, etc. An example of such a search for a model where the dark sector couples to the Standard Model via mixing with the Higgs boson (the so-called "scalar portal") is discussed in details in Section 5.

A different strategy can be adopted in experiments with electronic beam dumps (e.g. LDMX [173]) and some other non-collider experiments, e.g. experiments that study rare meson decays (e.g. NA62 [174]). In such experiments, it is possible to measure with high precision both the initial and final 4-momenta of all SM particles (except neutrinos). This allows to really search for missing energy rather than missing $p_{T}$ and significantly increase the sensitivity of DM searches (for small enough masses of DM particles). 


\section{Chapter 3}

\section{Self-interacting Dark Matter ${ }^{1}$}

\subsection{Theoretical description of SIDM}

\subsubsection{Qualitative picture: local equilibrium and $r_{\mathrm{SIDM}}$}

When Dark Matter particles scatter they change both directions and absolute values of their velocities. If DM density grows (e.g. in the center of a halo), collisions become more frequent and more effectively prevent particles from gathering near the center. One can expect a number of effects of DM self-interactions [110] including: (i) to isotropize the DM phase-space distribution $f$ and (ii) to form an region in the inner parts of the halo where the scatterings give rise to an effective pressure that compensates gravity, stops DM from further collapsing towards the center and DM density from growing. In this central region, we may expect the formation of an inner core with almost constant density. As we will see, this picture is roughly confirmed by simulations. For cross-sections $\sigma / m_{\chi} \sim$ $1 \mathrm{~cm}^{2} / \mathrm{g}$ the cores observed in simulations have quite noticeable sizes (a fraction of $r_{s}$ of the Navarro-Frenk-White (NFW) profile that fits the halo at large distances).

It is instructive to compare this behavior of simulated SIDM haloes with the properties of the hot gas in galaxy clusters. This gas is also a system with collisions, but with a much larger cross-section. The temperature and the density profile of intergalactic gas are reconstructed from observations of its X-ray emission. The observed behavior of gas is very different from what we see in SIDM simulations. Indeed, the temperature (velocity dispersion) of gas changes with radius. The density of gas does not exhibit a core, it is close to an isothermal density profile with $\rho \propto r^{-2}$ and therefore it is even cuspier than NFW (see e.g. [175, 176]).

This difference can be understood if we estimate the mean free path of DM particles between collisions, $\lambda$. We can easily check that for the case of SIDM with allowed crosssections we are in the weakly interacting regime, in the sense that the mean free path is large as compared to the size of the system. Let us estimate $\lambda$ e.g. for a typical dwarf

\footnotetext{
${ }^{1}$ In this section we largely follow papers I and II [28, 29]. Section 3.3 contains a new material, to be published in 2019.
} 
galaxy,

$$
\lambda \equiv \frac{1}{\sigma n_{\chi}} \simeq 4.8 \mathrm{kpc}\left(\frac{1 \mathrm{~cm}^{2} / \mathrm{g}}{\sigma / m_{\chi}}\right)\left(\frac{1 M_{\odot} / \mathrm{pc}^{3}}{\rho_{\chi}}\right) .
$$

This is clearly larger than the sub-kpc cores reported in dwarf galaxies, for realistic core densities $\rho_{\text {core }}=\mathcal{O}(1) M_{\odot} / \mathrm{pc}^{3}$ for dwarf galaxies [36] ( $\rho_{\text {core }}=\mathcal{O}(0.1) M_{\odot} / \mathrm{pc}^{3}$ for clusters [177]). For galaxies and galaxy clusters the situations are similar. Therefore, for such cross-sections, a DM particle typically only scatters at most a few times during the whole halo lifetime $t_{\text {age }}$ even if it may pass through the core region much more often.

These scatterings, nevertheless, change the velocity of particles and thus an equilibrium can be established in the inner part of the halo. In the outer parts of the halo, on the other hand, scatterings are so rare that the densities and velocities of DM particles would be very close to those of the CDM case. At large distances, we may expect therefore that the standard (NFW) DM profile should describe a SIDM halo well enough.

The simplest model $[95-97,107,118,178-180]$ is to assume that there exists a radius $r_{\text {SIDM }}$ such that:

- for $r>r_{\text {SIDM }}$ the density profile is well described by NFW,

- while for $r<r_{\text {SIDM }}$ we have an equilibrium.

Typically, scatterings first establish a local equilibrium inside a volume of the scale of a few mean free paths, establishing a global equilibrium in the whole system may take longer. Systems with large cross-sections and short (as compared to the size of the system) $\lambda$ contain many such volumes and therefore the temperature can be different in different parts of the system. This is the picture that is observed e.g. in clusters of galaxies (see Eq. (2.4) and related discussion in Section 2). For SIDM haloes, as we saw the mean free path is large as compared to the size of the system and there is only one equilibrium volume, outside this volume the density already drops and no equilibrium is established there.

The velocity dispersion established as a result of SIDM scatterings does not change inside $r_{\text {SIDM }}$.

This expectation is in a good agreement with simulations. To estimate the size of the region of equilibrium, $r_{\mathrm{SIDM}}$, one often assumes [105] that the average number of collisions per particle $\xi$ is of order one, i.e.

$$
\xi=\frac{L}{\lambda}=\frac{v_{\chi} t_{\mathrm{age}}}{\lambda}=\frac{\sigma}{m_{\chi}}\left\langle\rho_{\chi}\right\rangle_{\mathrm{SIDM}} v_{\chi} t_{\mathrm{age}} .
$$


Here $\left\langle\rho_{\chi}\right\rangle_{\mathrm{SIDM}}$ is the average density inside this region, $v_{\chi}=4 / \sqrt{\pi} \sigma_{\boldsymbol{v}}$ is the average relative velocity of the DM particles inside $r=r_{\text {SIDM }}$, and $\xi \gtrsim 1$ is an unknown number that we have introduced. This can be understood as a number of collisions that are needed to achieve the thermal equilibrium inside $r_{\text {SIDM }}$.

For constraining SIDM it is important to relate the observationally accessible core size of the haloes to the self-interaction cross-section. In particular, we need to find

1. the relation between $r_{\text {core }}$ and $r_{\mathrm{SIDM}}$,

2. the relation between $r_{\mathrm{SIDM}}$ and $\sigma / m_{\chi}$ for a given halo.

In the next subsections we will discuss an analytic model of the picture described above and will test this model using numerical simulations.

\subsubsection{Analytic description of SIDM haloes}

The dynamics of the self-interacting Dark Matter in the halo is governed by the Boltzmann equation,

$$
\frac{d f}{d t} \equiv \frac{\partial f}{\partial t}+\boldsymbol{v} \cdot \nabla f-\nabla \Phi \cdot \frac{\partial f}{\partial \boldsymbol{v}}=I_{\mathrm{coll}}
$$

where $f$ is a distribution function of DM particles and $I_{\text {coll }}$ is a collision integral that takes into account collisions between DM particles. To close the system of equations we need to add Poisson's equation,

$$
\Delta \Phi=4 \pi G \rho .
$$

As discussed above, a SIDM halo can be modeled by two regions (see [83] for a review):

- outside $r_{\text {SIDM }}$ collisions are insignificant and the collision integral in the Boltzmann equation can be neglected;

- inside $r_{\text {SIDM }}$ we assume that interactions are efficient enough to establish thermal equilibrium and the collision integral also vanishes.

The simplest model of the central part of the SIDM halo is to consider an isotropic steady state in the equilibrium. It is described by the Jeans equation (see Appendix A for its derivation from the Boltzmann equation),

$$
\frac{1}{3} \frac{\partial\left(\sigma_{v}^{2} \rho_{\chi}\right)}{\partial r}=-\rho_{\chi} \frac{\partial \Phi}{\partial r}
$$

where $\sigma_{\boldsymbol{v}}$ is the 3D velocity dispersion and $\rho_{\chi}$ is the density of Dark Matter particles. 

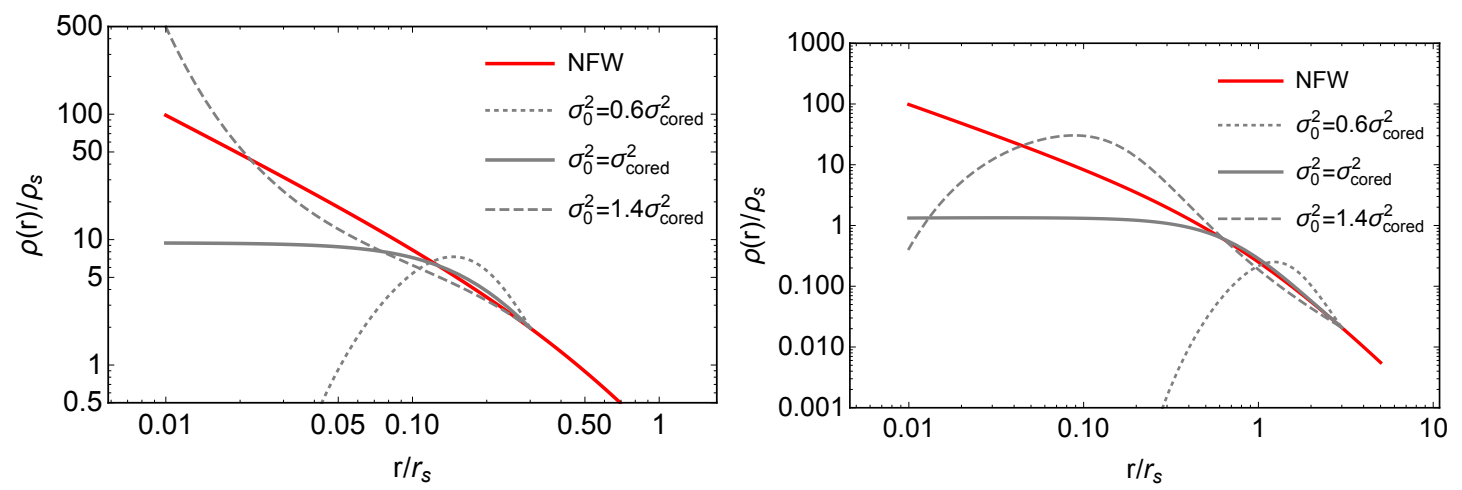

Figure 3.1: Different solutions of the Jeans equation connected to the NFW profile at radii $r_{\text {SIDM }}=0.3 r_{s}$ (left panel) and $r_{\text {SIDM }}=3 r_{s}$ (right panel) with fixed mass condition $M_{\text {iso }}\left(r_{\mathrm{SIDM}}\right)=M_{\mathrm{NFW}}\left(r_{\mathrm{SIDM}}\right)$.

Using Poisson's equation (3.4) (as well as neglecting the contribution of baryons to the gravitational potential) the Jeans equation simplifies to

$$
\frac{d}{d r}\left(\frac{r^{2}}{3 \rho_{\chi}} \frac{d\left(\sigma_{v}^{2} \rho_{\chi}\right)}{d r}\right)=-4 \pi G r^{2} \rho_{\chi} .
$$

As discussed above, for small cross-sections typical for SIDM, particles move freely inside $r_{\text {SIDM }}$ and the velocity dispersion is approximately constant in that region. With this condition, the Jeans equation becomes

$$
\frac{\bar{\sigma}_{v}^{2}}{3} \frac{d}{d r}\left(\frac{r^{2}}{\rho_{\chi}} \frac{d \rho_{\chi}}{d r}\right)=-4 \pi G r^{2} \rho_{\chi}, \quad r<r_{\mathrm{SIDM}},
$$

where the constant $\bar{\sigma}_{\boldsymbol{v}}$ describes the average value of the velocity dispersion $\sigma_{\boldsymbol{v}}$ inside $r_{\text {SIDM }}$.

The Jeans equation has solutions with different asymptotic behaviour in the center, see examples in Fig. 3.1. We consider the solutions where the density goes to zero as clearly unphysical. There are two classes of physical solutions: solutions with a constant density in the center and solutions with a density cusp in the center. For example, it is easy to check explicitly that $\rho(r)=\sigma_{\boldsymbol{v}}^{2} /\left(2 \pi G r^{2}\right)$ satisfies Eq. (3.7). This solution is called in the literature "the isothermal sphere" [46]. It describes systems where the temperature is a constant but the mean free path is much smaller than the size of the system. As discussed above, this is the physical solution for cross-sections much larger than what we are interested in here. Therefore, we will not consider such solutions ${ }^{2}$.

\footnotetext{
${ }^{2}$ We note that a solution with a thermalized inner core in SIDM haloes is only a quasi-stable configuration. Given enough time, collisions eventually will result in the well-known gravothermal catastrophe, studied in globular clusters [181]. The collapse of the core leads to a central density profile that is even cuspier than in CDM haloes $[182,183]$. For this process to be relevant within a Hubble time, however, large
} 
The class of solutions that is relevant for our purposes describes roughly a constant density at small radii, $\rho_{\chi}^{\prime}(0)=0$, and approach the isothermal sphere at larger $r$. The so-called pseudo isothermal sphere,

$$
\rho_{\mathrm{ISO}}(r)=\frac{\rho_{0}}{1+r^{2} / r_{0}^{2}},
$$

is a very good approximation to this class of solutions for the Jeans equation, at least for $r \ll r_{0}$ and $r \gg r_{0}$.

As our goal is to build a model for weakly interacting SIDM as observed in simulations, we will consider only the solutions with constant density in the center and will, therefore, impose the condition $\rho^{\prime}(0)=0$.

The second-order Jeans equation (3.7) inside $r_{\text {SIDM }}$ requires two boundary conditions. Additionally, we need to fix the free parameter $\sigma_{\boldsymbol{v}}$ that can be done imposing $\rho^{\prime}(0)=0$, as for SIDM we limit ourselves to the solutions with a core. Below we will discuss possible boundary conditions and will verify them by direct comparison with simulation data.

Gluing with CDM. ${ }^{3}$ Outside $r_{\text {SIDM }}$ there is no reason to assume that $\sigma_{v}$ is a constant as particles do not scatter and do not exchange their velocities. Instead, we assume that the density profile $\rho(r)$ is the same as for collisionless CDM. When modeling simulated haloes, we will take this $\rho(r)$ from CDM simulations, for the observed objects this will be a NFW profile that fits the data at large radii. The Jeans equation (3.5) becomes an equation for the velocity dispersion $\sigma_{\boldsymbol{v}}(r)$.

Then to build a solution for all radii, we need to glue together a solution of the Jeans equation for a constant $\sigma_{\boldsymbol{v}}$ for $r<r_{\mathrm{SIDM}}$ with e.g. $\rho_{\mathrm{NFW}}(r)$ for $r>r_{\mathrm{SIDM}}$. Let us assume that scatterings between DM particles do not push them outside $r_{\text {SIDM }}$, but only re-distribute them inside this radius. This means that we can choose, as the first boundary condition, the requirement that the mass at $r_{\text {SIDM }}$ is the same in the SIDM halo as in the CDM halo.

Our first assumption is therefore that the radius where the masses are equal roughly coincides with the radius $r_{\text {SIDM }}$ where the velocity dispersion becomes constant

(I). $\quad M_{\mathrm{SIDM}}\left(r_{\mathrm{SIDM}}\right)=M_{\mathrm{CDM}}\left(r_{\mathrm{SIDM}}\right)$.

cross-sections $\gtrsim 10 \mathrm{~cm}^{2} / \mathrm{g}$ are required, so this regime is not relevant for the purposes of our work.

${ }^{3}$ Below, till section 3.2.7, we follow paper II [29] 
In the literature it is often assumed that the second condition at $r_{\text {SIDM }}$ is that the density is continuous $\rho_{\mathrm{SIDM}}\left(r_{\mathrm{SIDM}}\right)=\rho_{\mathrm{CDM}}\left(r_{\mathrm{SIDM}}\right)$. This condition, however, is not at all obvious. Indeed, to derive the Jeans equation we need to assume that the collision integral in the Boltzmann equation is zero. In our model, as discussed above, the collision integral is equal to zero on both sides of $r_{\text {SIDM }}$ (for different reasons on each side). In reality, however, there exists an intermediate layer around $r_{\text {SIDM }}$ where the collisions frequency is not sufficient to establish an equilibrium, but the collisions cannot be neglected completely.

When we assume that the Jeans equation is valid on both sides of $r_{\text {SIDM }}$, we imply that the width of this intermediate layer is much smaller than $r_{\text {SIDM }}$.

It is clear that in the approximation that we use we can not demand that all quantities will be continuous at $r_{\text {SIDM }}$.

There is, therefore, no reason to assume that mass and density will be equal for SIDM and $\mathrm{CDM}$ at the same radius (and, in particular, at $r_{\mathrm{SIDM}}$ ). We will see in the simulations that indeed, the densities are not the same at $r_{\text {SIDM }}$. We will assume therefore an alternative second boundary condition. Namely, let us introduce the total kinetic energy of DM particles inside radius $r$ defined as

$$
E_{\mathrm{kin}}(r)=2 \pi \int_{0}^{r} \rho_{\mathrm{CDM}}(r) \sigma_{\boldsymbol{v}}^{2}(r) r^{2} d r .
$$

Instead of continuous density, we will assume as the second boundary condition that the kinetic energies inside $r_{\text {SIDM }}$ are equal for CDM and SIDM

(II). $\quad E_{\mathrm{kin}}^{\mathrm{SIDM}}\left(r_{\mathrm{SIDM}}\right)=E_{\mathrm{kin}}^{\mathrm{CDM}}\left(r_{\mathrm{SIDM}}\right)$.

These two boundary conditions for the Jeans equation are sufficient to choose a unique solution with a constant density at the center (the value of $\bar{\sigma}_{\boldsymbol{v}}$ is additionally fixed by the requirement of $\left.\rho(0)^{\prime}=0\right)$.

\subsection{Testing the theory with simulations}

In this section, we use 28 simulations of DM-only galaxy clusters [124]. The mass range for these cluster-sized haloes is $\mathrm{M}_{200} \approx 0.5-1.9 \times 10^{15} \mathrm{M}_{\odot} h^{-1}$. All 28 haloes were simulated in CDM and SIDM cosmologies with cross-sections $\sigma / m=0.5 \mathrm{~cm}^{2} / \mathrm{g}$ and $\sigma / m=1 \mathrm{~cm}^{2} / \mathrm{g}$. Also, 10 haloes were simulated with cross-sections $\sigma / m=5 \mathrm{~cm}^{2} / \mathrm{g}$ and $\sigma / m=10 \mathrm{~cm}^{2} / \mathrm{g}$ (see [124] and paper II [29] for details). 

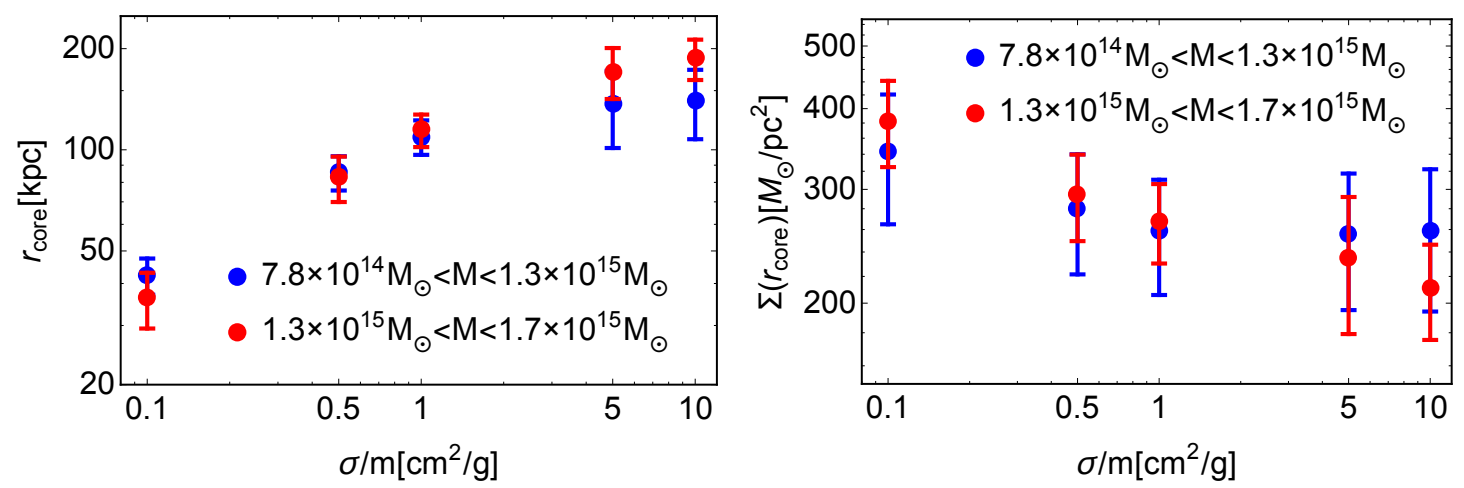

Figure 3.2: The dependence of the SIDM surface density in the core (right panel) and the core radius (left panel) on the cross-section. Error bars represent the scatter in our suite of simulated haloes. The data is split into 2 approximately equal mass bins. Taken from paper II [29].

\subsubsection{Main properties of simulated SIDM haloes}

The general expectation that SIDM haloes should develop cores is confirmed in all our simulated SIDM haloes. As shown in the left panel of Fig. 3.2 we also confirm that the core radius should grow with the interaction strength.

In this work we define the core radius $r_{\text {core }}$ as: $\rho_{\text {SIDM }}(0)=\rho_{\text {CDM }}\left(r_{\text {core }}\right)$.

This definition of the core radius does not depend on the parametric model of the core profile used to fit the data. An observable quantity that allows using in our analysis objects of very different sizes is the surface density $\Sigma$ inside the core radius. $\Sigma$ is defined as in paper I [28]

$$
\Sigma(r)=\frac{3 M(r)}{4 \pi r^{2}} .
$$

In the right panel of Fig. 3.2 we show this quantity as a function of the interaction strength. In Fig. 3.2 we have grouped both $r_{\text {core }}(\sigma / m)$ and $\Sigma(\sigma / m)$ into two mass bins. We see that both quantities saturate around $\sigma / m \sim 5 \mathrm{~cm}^{2} / \mathrm{g}$ and the maximal $r_{\text {core }}$ is larger for haloes with larger masses.

These plots allow us to make immediately a couple of important observations

- The core radius depends weakly on the interaction strength. This means that a small error in the measurement of the core radius gives rise to a significant error for the cross-section.

- It is completely impossible to constrain cross-sections larger than $\sigma / m \gtrsim 5 \mathrm{~cm}^{2} / \mathrm{g}$.

These conclusions are derived from simulation results and do not imply any analytical model to describe DM self-interactions. 


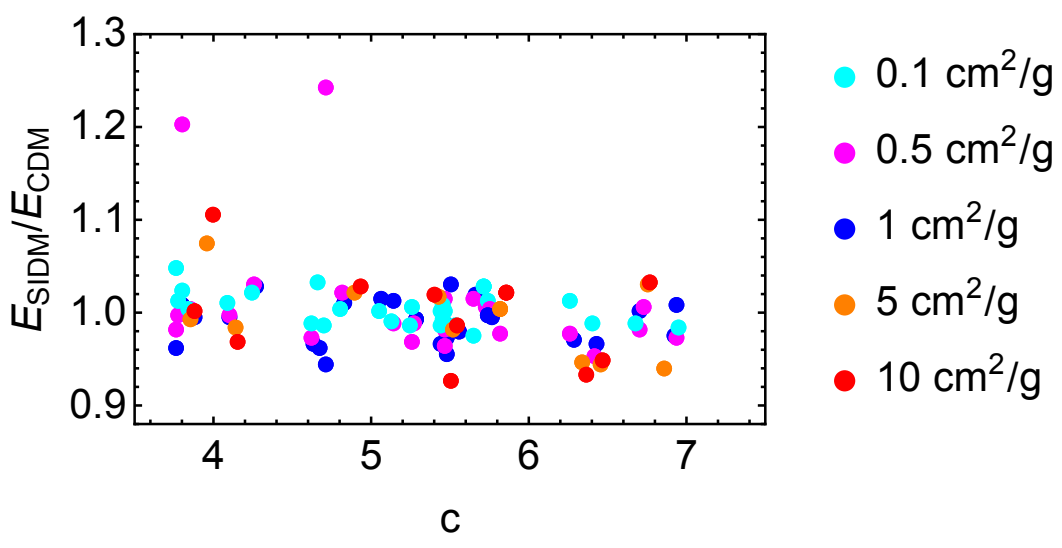

Figure 3.3: The ratio of the kinetic energies of SIDM and CDM haloes as a function of halo concentration. Taken from paper II [29].

\subsubsection{Testing the model assumptions by $\mathrm{N}$-body simulations}

To verify the analytic simple model formulated above, we will explicitly test whether at the same radius $r_{\text {SIDM }}$

1. masses of CDM and SIDM haloes simulated with the same initial conditions are equal to each other inside $r_{\text {SIDM }}$;

2. the total kinetic energies of CDM and SIDM particles are the same;

3. for the SIDM case, the velocity dispersion is flat inside $r_{\text {SIDM }}$.

Below we will demonstrate that in our simulations such a radius indeed exists for all haloes. Then we will solve the Jeans equation (3.7) with the above-mentioned boundary conditions at $r_{\text {SIDM }}$ and compare the results with the density profiles of the simulated SIDM haloes.

We start by finding the radius $r_{\mathrm{SIDM}} \equiv r_{M}$ (radius inside which the masses in SIDM and $\mathrm{CDM}$ are equal) for all simulated haloes.

Next we compare the total kinetic energies inside these radii. The ratio of total kinetic energies from SIDM and CDM simulations is shown in Fig. 3.3 as a function of the halo concentration, $c=r_{200} / r_{s}$ (see Section 3.2.8 for details).

In Fig. 3.3 we observe that the kinetic energies of SIDM and CDM profiles inside radius $r_{M}$ are equal up to $\lesssim 5 \%$ difference for most of the objects. 


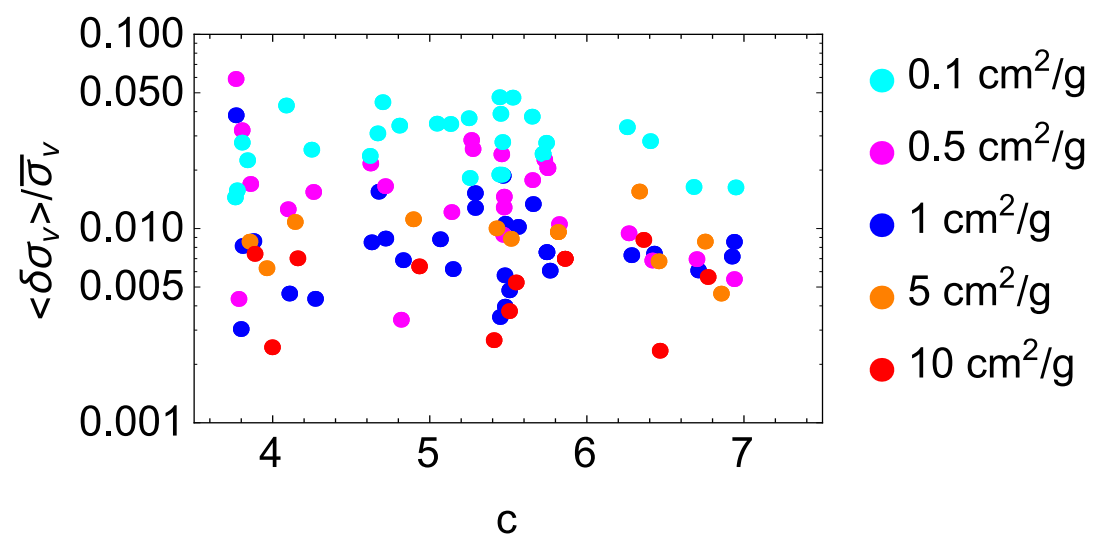

Figure 3.4: The average deviation of the total velocity dispersion inside $r_{M}$ from the best fit constant value $\bar{\sigma}_{\boldsymbol{v}}$ in SIDM simulations with different cross-sections $\sigma / m$, as a function of halo concentration. Taken from paper II [29].

Next we check that the SIDM velocity dispersion is constant inside $r_{M}$. Fig. 3.4 shows the deviation of the velocity dispersion from the best fit constant value $\bar{\sigma}_{\boldsymbol{v}}$, averaged inside $r_{M}$ as $\left\langle\delta \sigma_{\boldsymbol{v}}\right\rangle=\left\langle\left|\sigma-\bar{\sigma}_{\boldsymbol{v}}\right|\right\rangle$, as a function of halo concentration.

For most of the haloes the velocity dispersion is constant up to a deviation $\lesssim 5 \%$ for $\sigma / m \leq 1$ and $\lesssim 1 \%$ for $\sigma / m \geq 1 \mathrm{~cm}^{2} / g$.

The equality of kinetic energies and masses can be used to predict the velocity dispersion $\bar{\sigma}_{\boldsymbol{v}}$ for SIDM haloes. The kinetic energy is given by

$$
E_{\mathrm{kin}}^{\mathrm{CDM}}=\sum_{\text {particles }} m_{i} \frac{v_{i}^{2}}{2} .
$$

For CDM we can calculate this quantity directly for all particles inside $r_{M}$ from simulations and obtain $E_{\mathrm{kin}}^{\mathrm{CDM}}\left(r_{M}\right)$. For SIDM we assume that in average we can substitute $v_{i}^{2}=\bar{\sigma}_{\boldsymbol{v}}^{2}$ and the kinetic energy becomes

$$
E_{\mathrm{kin}}^{\mathrm{SIDM}}=\sum_{\text {particles }} m_{i} \frac{\bar{\sigma}_{\boldsymbol{v}}^{2}}{2}=\frac{\bar{\sigma}_{\boldsymbol{v}}^{2}}{2} \sum_{\text {particles }} m_{i}=M\left(r_{M}\right) \frac{\bar{\sigma}_{\boldsymbol{v}}^{2}}{2} .
$$

The equality of kinetic energies and masses inside $r_{M}$ means that $E_{\mathrm{kin}}^{\mathrm{CDM}}\left(r_{M}\right)=M_{\mathrm{CDM}}\left(r_{M}\right) \frac{\bar{\sigma}_{\boldsymbol{v}}^{2}}{2}$ and we can predict

$$
\left(\bar{\sigma}_{\boldsymbol{v}}^{\text {pred }}\right)^{2}=\frac{2 E_{\mathrm{kin}}^{\mathrm{CDM}}\left(r_{M}\right)}{M_{\mathrm{CDM}}\left(r_{M}\right)} .
$$

We test this prediction with the simulation data for $\sigma / m=1 \mathrm{~cm}^{2} / \mathrm{g}$ in Fig. 3.5. Again, for 

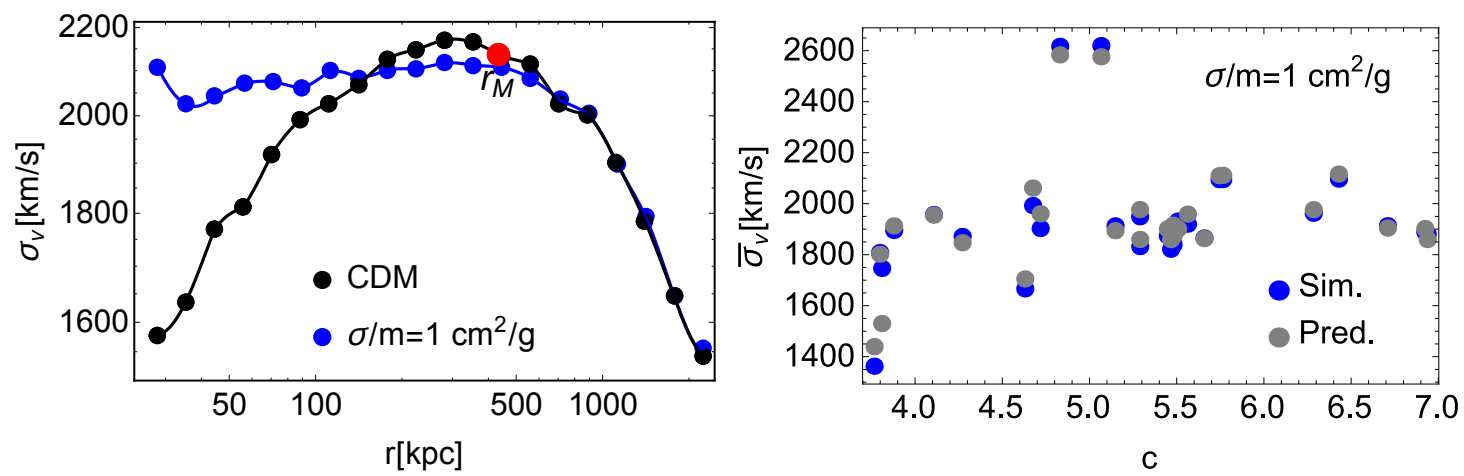

Figure 3.5: Left panel: An example of the velocity dispersion profile for a SIDM halo (blue) $\left(\sigma / m=1 \mathrm{~cm}^{2} / \mathrm{g}\right)$ and CDM (black) simulated for the same initial conditions. Right panel: The average value of the velocity dispersion inside $r_{M}$ for simulated SIDM haloes (blue) and the predicted values (gray) obtained from the equality of masses and kinetic energies $\left(\sigma / m=1 \mathrm{~cm}^{2} / \mathrm{g}\right)$. Taken from paper II [29].

most of the haloes the predicted value is very close to what we see in simulations.

We can see that the predicted value of the velocity dispersion is quite good for most haloes.

We conclude that $r_{M}$ is a good proxy for $r_{\text {SIDM }}$ and the boundary requirement of equal kinetic energy, chosen as a second boundary condition provides a good prediction for the value of constant velocity dispersion of SIDM haloes.

We have all the necessary ingredients that allow us to make predictions for the inner density profiles of the SIDM haloes and test these predictions with our simulations.

\subsubsection{Testing predictions for the cores}

Now we will use the Jeans equation with our boundary conditions to predict the inner density profile for SIDM. An example of a predicted SIDM density profile for $\sigma / m=1$ $\mathrm{cm}^{2} / \mathrm{g}$ is given in the left panel of Fig. 3.6.

We see that the prediction is not very good - the density in the core is lower than the real density of the simulated halo. It is convenient to introduce the quantity of $\kappa$

$$
\kappa=\frac{\langle\rho\rangle_{c}}{\langle\rho\rangle_{M}}
$$

where $\langle\rho\rangle_{c}$ and $\langle\rho\rangle_{M}$ are the average densities inside the core and inside $r_{M}$ respectively. The comparison between predicted and simulated $\kappa$ is shown in the right panel of Fig. 3.6 (for the case of $\sigma / m=1 \mathrm{~cm}^{2} / \mathrm{g}$ ). 

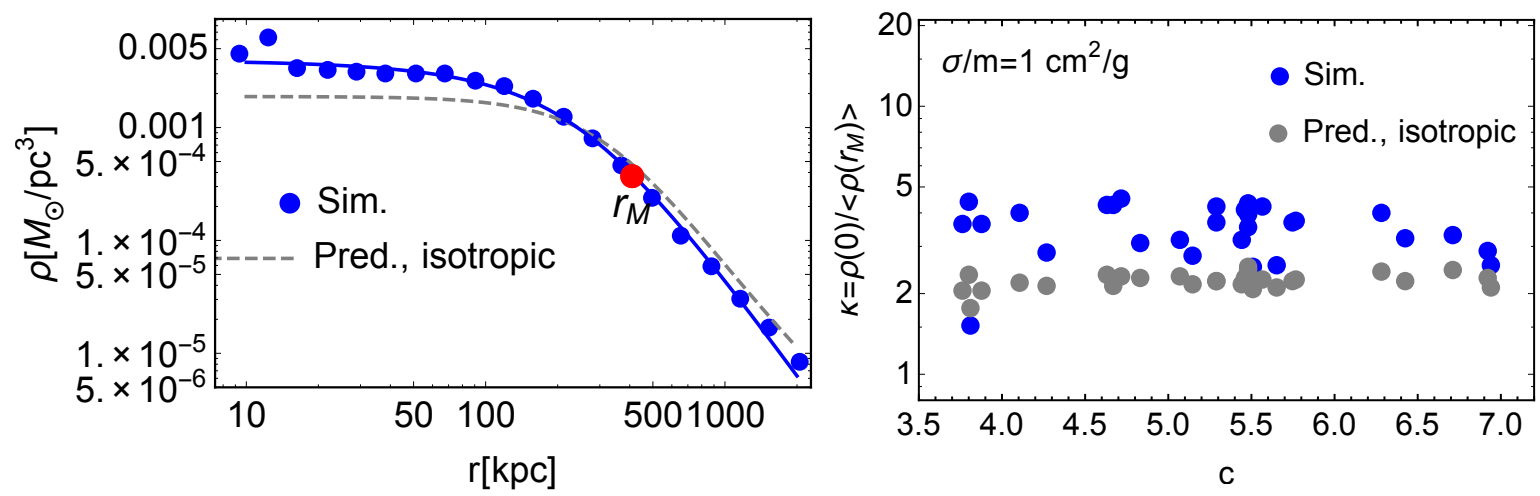

Figure 3.6: Left panel: Density profiles for a SIDM halo with $\sigma / m=1 \mathrm{~cm}^{2} / \mathrm{g}$ from simulations (blue) and the prediction from isotropic Jeans equation (dashed gray line). Right panel: Ratio between simulated haloes with $\sigma / m=1 \mathrm{~cm}^{2} / \mathrm{g}$ (blue) and predicted with the isotropic Jeans equation (gray) central density as a function of halo concentration. Taken from paper II [29].

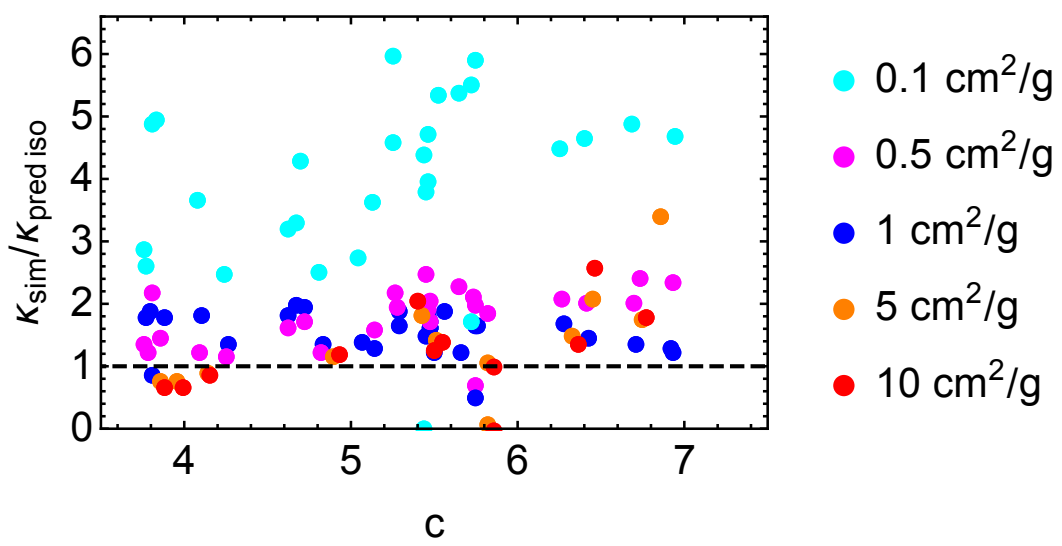

Figure 3.7: The ratio of simulated and predicted (isotropic Jeans equation) values of $\kappa=$ $\rho(0) /\left\langle\rho\left(r_{M}\right)\right\rangle$, for different values of self-interaction cross-sections $\sigma / m$. Taken from paper II [29].

The density predicted with the Jeans equation is systematically lower as compared to the simulations. The average value $\langle\kappa\rangle_{\text {sim }} /\langle\kappa\rangle_{\text {pred }}=1.6$. A similar behaviour is observed for other cross-sections as well (see Fig. 3.7). We will see in the next section that the reason for this systematic discrepancy is anisotropy in the velocity dispersion that appears to be surprisingly large for the simulated SIDM haloes. 

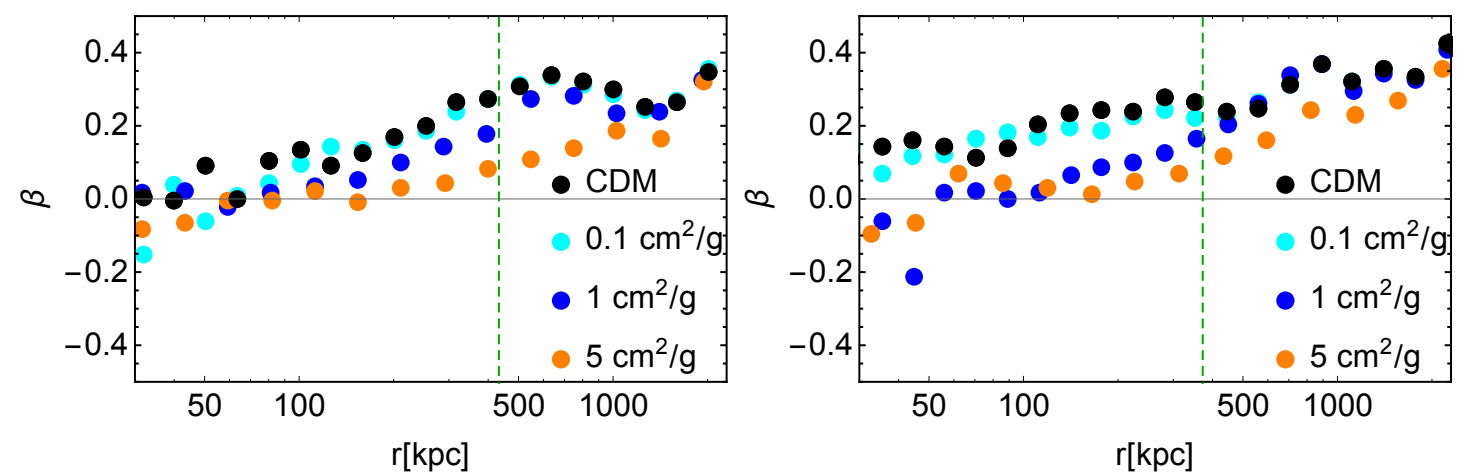

Figure 3.8: Velocity anisotropy $\beta(r)=1-\left(\sigma_{\theta}^{2}+\sigma_{\phi}^{2}\right) /\left(2 \sigma_{r}^{2}\right)$, cyan, blue and orange for $\sigma / m=0.1,1$ and $5 \mathrm{~cm}^{2} / \mathrm{g}$, black for CDM. The vertical green dashed line indicates $r_{M}$, left and right panels are two different simulated haloes. Taken from paper II [29].

\subsubsection{Anisotropic velocities and improved prediction for the inner den- sity}

In a perfect kinetic equilibrium, the velocities distribution should be isotropic and therefore the anisotropy of velocity dispersion $\beta$

$$
\beta(r)=1-\frac{\sigma_{\theta}^{2}+\sigma_{\phi}^{2}}{2 \sigma_{r}^{2}}
$$

should be equal to zero.

In the SIDM haloes, the equilibrium may not be perfect - it can be established only in the relatively small inner part and particles from outside that region can enter and leave it.

Indeed, in SIDM simulations, we can see (Fig. 3.8) that the anisotropy for SIDM haloes is not negligible at $r_{M}$ (and close to its values for CDM), at least for $\sigma / m \lesssim$ $1 \mathrm{~cm}^{2} / \mathrm{g}$.

This means that to describe the SIDM density one needs to use the anisotropic Jeans equation (see [175] and Appendix A)

$$
\frac{d}{d r}\left(\frac{r^{2}}{\rho} \frac{d}{d r}\left(\rho \sigma_{r}^{2}\right)+2 r \beta \sigma_{r}^{2}\right)=-4 \pi G r^{2} \rho
$$

with the radial velocity dispersion $\sigma_{r}$. Nevertheless, we see that in the simulations the total velocity dispersion $\sigma_{\boldsymbol{v}}$ is very close to a constant. We can express $\sigma_{r}$ via this constant and anisotropy $\beta$ as

$$
\sigma_{\boldsymbol{v}}^{2} \equiv \sigma_{r}^{2}+\sigma_{\theta}^{2}+\sigma_{\phi}^{2}=\sigma_{r}^{2}(3-2 \beta) .
$$




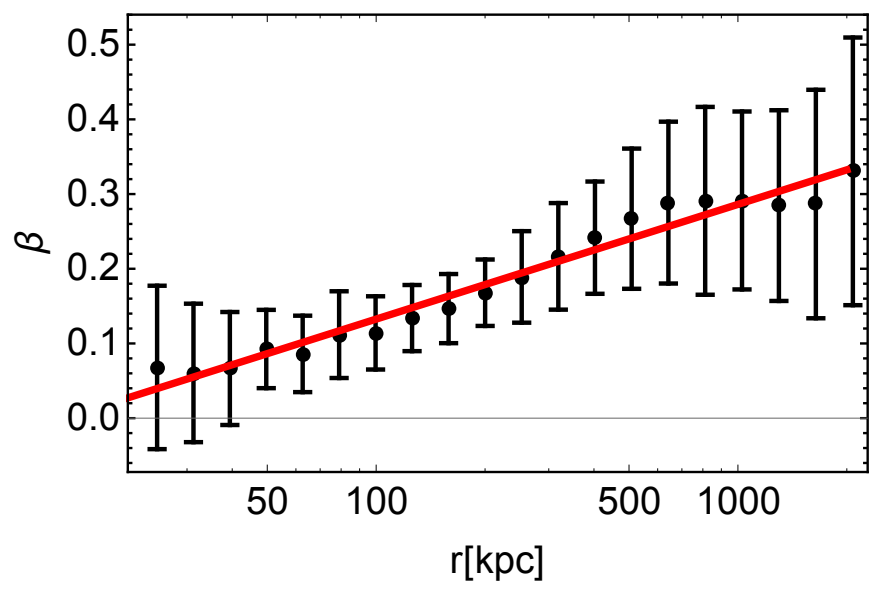

Figure 3.9: Velocity anisotropic profiles for CDM haloes. Error bars represent the standard deviation. The red line is the best fit with the ansatz (3.18) (see Table 3.1). Taken from paper II [29].

\begin{tabular}{|c|c|c|}
\hline \multicolumn{3}{|c|}{ Best fit values of $\langle\beta\rangle=A \ln \left(r / r_{\beta}\right)$} \\
\hline$\sigma / m\left[\mathrm{~cm}^{2} / \mathrm{g}\right]$ & $A$ & $r_{\beta}[\mathrm{kpc}]$ \\
\hline $\mathrm{CDM}$ & 0.067 & 13.7 \\
0.1 & 0.074 & 21.8 \\
0.5 & 0.089 & 50.1 \\
1 & 0.102 & 76.4 \\
5 & 0.124 & 194 \\
10 & 0.166 & 387 \\
\hline
\end{tabular}

Table 3.1: The best fit parameters for the anisotropy profile $\beta(r)$ (Eq. 3.18) from simulated haloes for different cross-sections.

To solve Eq. (3.16) we need the anisotropy profile $\beta(r)$ as an input. In our simulations, we know $\beta(r)$ for each object. However, for the real observed objects, the velocity anisotropy of DM particles is not directly observable. We tried to use simulations in such a way that it can be applied to real objects. Namely, we use a simple ansatz

$$
\langle\beta(r)\rangle=\left\{\begin{array}{cl}
A \ln \left(r / r_{\beta}\right), & \text { for } r \geq r_{\beta} \\
0, & \text { for } r<r_{\beta}
\end{array}\right.
$$

to fit $\beta(r)$ for all haloes for a given cross-section (see Fig. 3.9). The best fit values of $A$ and $r_{\beta}$ are given in Table 3.1.

Solving Eq. (3.16) with an ansatz $\langle\beta(r)\rangle$ we see that by taking into account anisotropy, we significantly improve the accuracy of the predictions as compared to what have been used in the literature (see Fig. 3.10 and Fig. 3.11). 

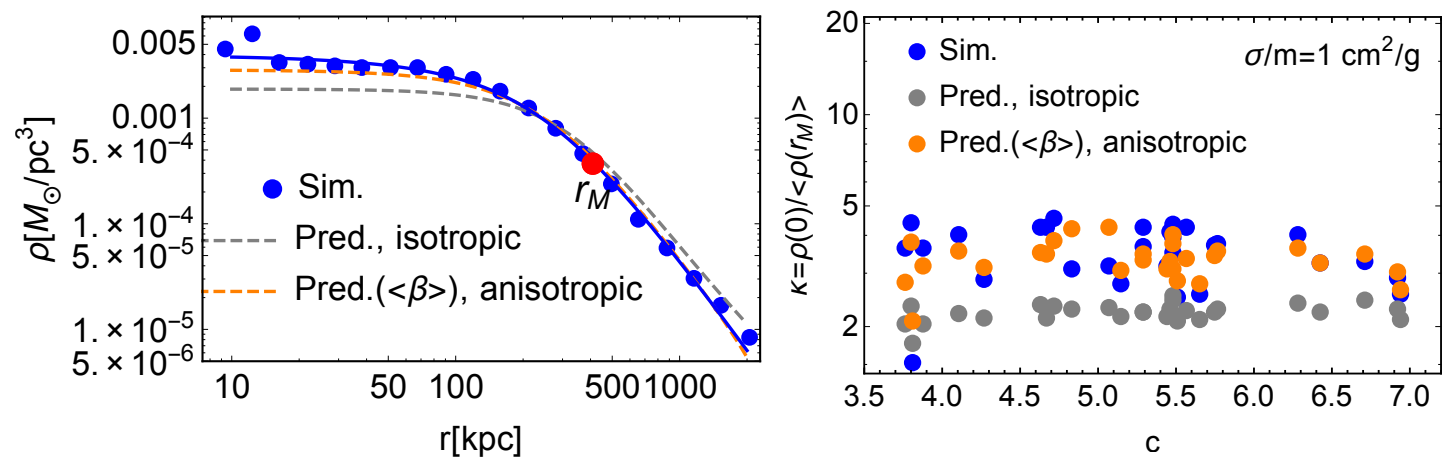

Figure 3.10: Left panel: Density profiles for a SIDM halo with $\sigma / m=1 \mathrm{~cm}^{2} / \mathrm{g}$. Blue points - simulations, dashed gray line - prediction of isotropic model, orange line - anisotropic model. $r_{M}$ is marked by the red dot. Right panel: $\kappa=\rho(0) /\left\langle\rho\left(r_{M}\right)\right\rangle$ for $\sigma / m=1 \mathrm{~cm}^{2} / \mathrm{g}$ as a function of halo concentration from the simulation data (blue), and the isotropic/anisotropic Jeans equation (gray/orange). Taken from paper II [29].
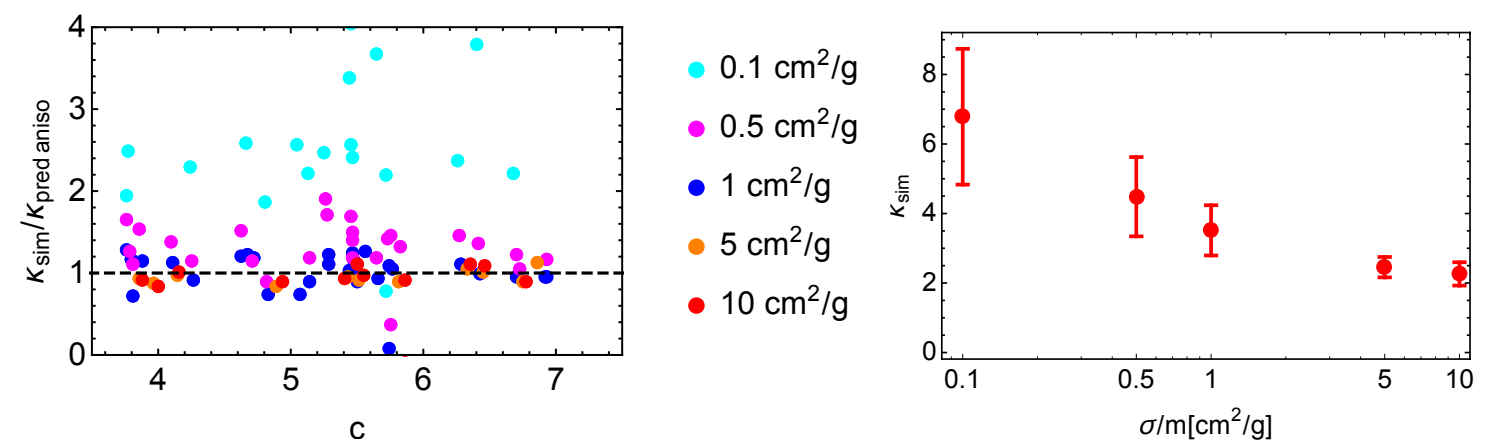

Figure 3.11: Left panel: The ratio of the simulated and predicted (anisotropic Jeans equation) values of $\kappa$ as a function of halo concentration for different values of the selfinteraction cross-section $\sigma / m$. Right panel: The mean values $\kappa_{\text {sim }}=\rho(0) /\left\langle\rho\left(r_{M}\right)\right\rangle$ with the standard deviation in simulated SIDM haloes as a function of the self-interaction crosssection $\sigma / m$. Taken from paper II [29].

For $\sigma / m \sim 0.1 \mathrm{~cm}^{2} / \mathrm{g}$ the difference between our predictions and the simulations is a bit larger. This is because for so small cross-sections the equilibrium is even less established.

\subsubsection{Predictions based on NFW fits}

For the real objects, we can not make predictions for the inner density profile of SIDM haloes based on input from CDM simulations. Instead, we have to assume that the halo shape is the same for CDM and SIDM at large radii. Then we can use NFW fits at large $r$ in the same way we use CDM simulation data here. At these distances, we usually have smaller contributions from baryons and can trust our data about the DM density profile. 

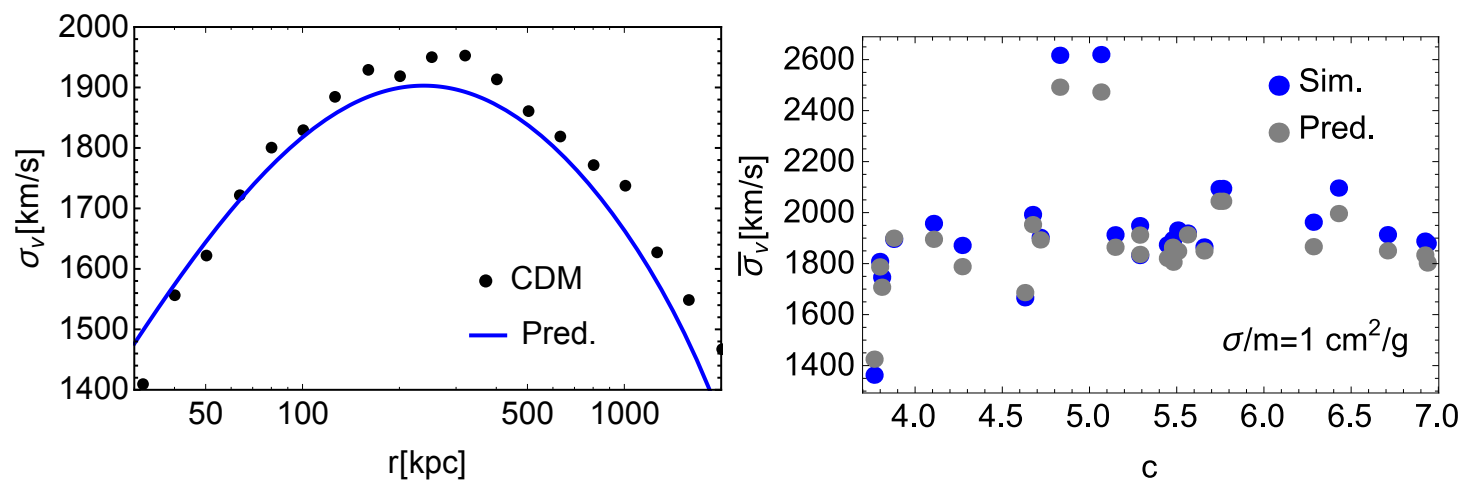

Figure 3.12: Left panel: Total velocity dispersion profile for a simulated CDM halo (black points) and the predictions from the anisotropic Jeans equation with NFW input (blue line). Right panel: Constant velocity dispersion, $\bar{\sigma}_{\boldsymbol{v}}$, of simulated SIDM haloes $(\sigma / m=1$ $\mathrm{cm}^{2} / \mathrm{g}$ ) inside $r_{M}$ as a function of concentration (blue), and predicted values (gray). Taken from paper II [29].

In this section, we will try this approach using simulated haloes and fitting them by NFW at large radii. To use the equal kinetic energy boundary conditions at $r_{M}$ we have to find also the velocity dispersion. For this we solve the anisotropic Jeans equation (see Appendix B) for $\sigma_{\boldsymbol{v}}$ using our NFW fits as an input. For the anisotropy $\beta(r)$ we will use the ansatz (3.18), see Table 3.1. An example of the solution for $\sigma_{\boldsymbol{v}}(r)$ is presented in Fig. 3.12 (left panel).

Then we can calculate the total kinetic energy of CDM inside $r_{M}$ and, therefore, the constant $\sigma_{v}^{\text {SIDM }}$ for the SIDM hallo, see Fig. 3.12 (right panel).

Then we can repeat the same procedure as above to predict the inner density profile of SIDM. In Fig. 3.13 we see the comparison between the values of $\kappa$ predicted as described here and the values taken from simulations. The results obtained from the NFW fits are as good as in Fig. 3.11, where CDM simulations were used as an input.

\subsubsection{Predicting the radius $r_{M}$ for a given cross-section}

So far we used $r_{M}$ as an input for our analysis and took it directly from simulations. If we want to use our findings to constraint SIDM, we need to find an explicit relation between $r_{M}$ and the cross-section.

In the literature in order to fix this relation it is often assumed that inside $r_{\text {SIDM }}$ the particles have in average one collision for the halo lifetime $t_{\text {age }}$ (see $[83,105]$ and references therein):

$$
N(r) \equiv \frac{\sigma}{m} \rho(r) v(r) t_{\mathrm{age}}
$$



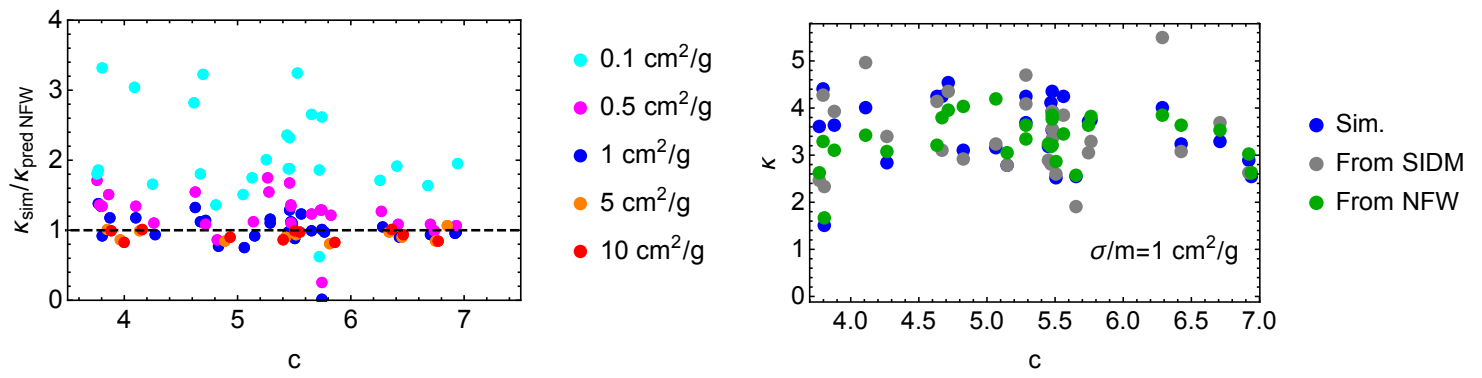

Figure 3.13: Left panel: The ratio of simulated and predicted (the anisotropic Jeans equation with NFW as an input) $\kappa$ as a function of halo concentration. Right panel: Simulated (blue), predicted using input of CDM simulations (gray) and predicted using NFW input (green) $\kappa$ as a function of halo concentrations for $\sigma / m=1 \mathrm{~cm}^{2} / \mathrm{g}$. Taken from paper II [29].

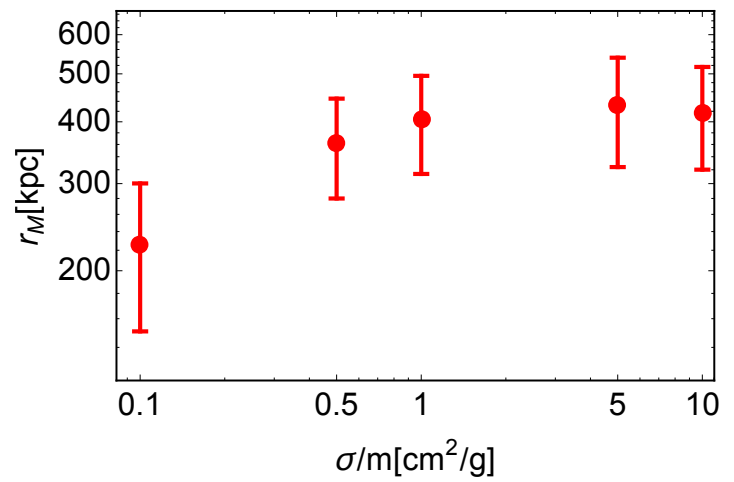

Figure 3.14: The radius $r_{M}$ (with the standard deviation of the ensemble) as a function of the self-interaction cross-section. Taken from paper II [29].

and

$$
N\left(r_{\mathrm{SIDM}}\right)=\frac{\sigma}{m} \rho\left(r_{\mathrm{SIDM}}\right) v\left(r_{\mathrm{SIDM}}\right) t_{\mathrm{age}}=1,
$$

where $v(r)$ is the average relative velocity of DM particles at radius $r$ that we estimate (assuming the Maxwell distribution) as $v(r)=(4 / \sqrt{3 \pi}) \sigma_{\boldsymbol{v}}^{\mathrm{SIDM}}(r)$, and $t_{\mathrm{age}}$ is defined as the half-mass formation time (see paper I [28]).

In this section, we will explicitly verify this relation using simulations. In Fig. 3.15 we show the average number of collisions per particle at radius $r_{M}$ and notice that $N_{\text {sim. }}\left(r_{M}\right)$ varies significantly in our simulation suite. In the bottom panel of Fig. 3.16 we show typical examples of the radial dependence of the number of collisions, $N(r)$, found in the simulations. 

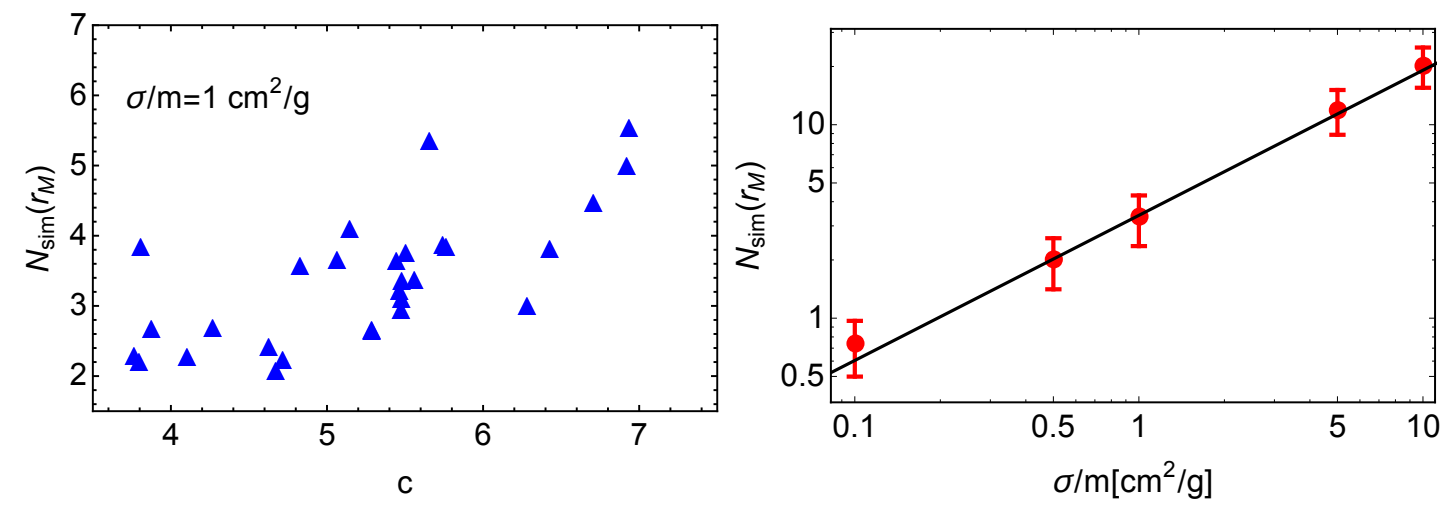

Figure 3.15: The average number of collisions at radius $r_{M}$ (left panel) as a function of concentration for $\sigma / m=1 \mathrm{~cm}^{2} / \mathrm{g}$ and as a function of the cross-section (right panel), fitted by $N\left(r_{M}\right) \propto(\sigma / m)^{0.75}$. Taken from paper II [29].
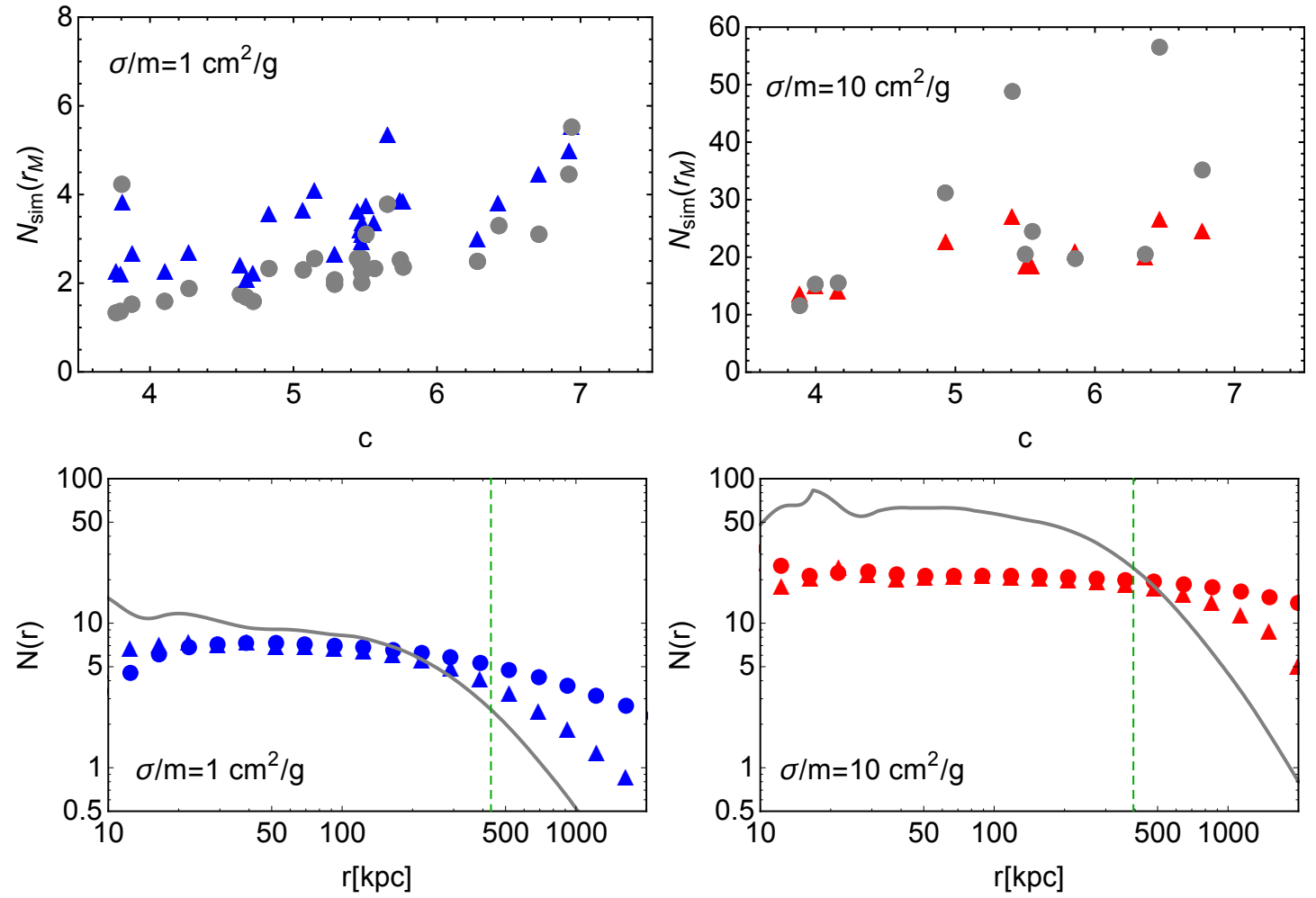

Figure 3.16: The average number of collisions inside (dots) and at radius $r_{M}$ (triangles), as a function of concentration for $\sigma / m=1 \mathrm{~cm}^{2} / \mathrm{g}$ (top left) and $10 \mathrm{~cm}^{2} / \mathrm{g}$ (top right). The lower panels show the number of collisions inside and at $r$ for $\sigma / m=1 \mathrm{~cm}^{2} / \mathrm{g}$ (lower left) and for $10 \mathrm{~cm}^{2} / \mathrm{g}$ (lower right). Gray dots and lines represent the predictions of Eq. (3.19). The green vertical line indicates $r_{M}$. Taken from paper II [29]. 

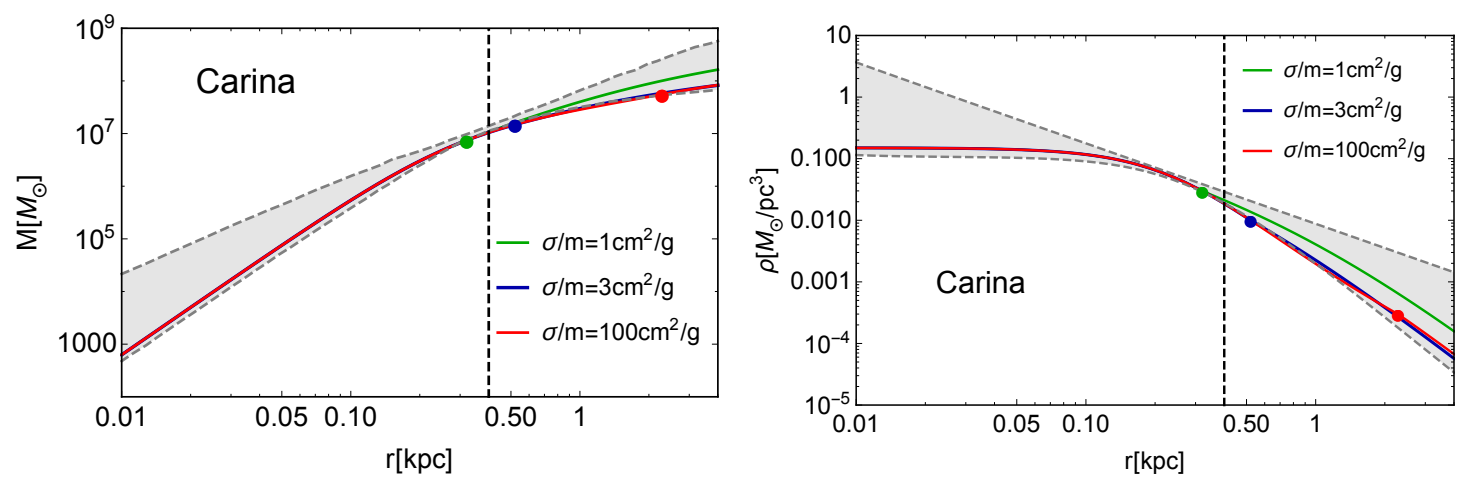

Figure 3.17: The mass (left) and the density (right) profiles for Carina [185]. The shaded regions represent the observational uncertainty. Green, blue and red lines are the profiles obtained using the approach of Ref. [105] for different cross-sections. The colored dots represent $r_{\text {SIDM }}$ for the corresponding $\sigma / m$ and the dashed vertical line indicates the halflight radius. Taken from paper I [28].

We conclude that Eq. (3.19) does not predict the number of collision per particle correctly.

\subsubsection{Connecting $r_{\text {core }}$ and $r_{\text {SIDM }}$ by fitting rotation curve or density profile}

Before continuing, let us comment on why it is so challenging to directly relate the core radius $r_{\text {core }}$ to the cross-section of self-interaction. It has been argued [83, 100, 105, $106,131,184]$ that this question can be fully resolved for each halo by (i) defining the scale $r_{\text {SIDM }}$ using the "one collision per particle" condition; (ii) fitting the solution of the isotropic Jeans equation inside this $r_{\text {SIDM }}$ to the data (rotation curve, velocity dispersion etc). Let us try to apply this procedure. In this section we follow paper I [28]. As a reminder of our discussion in Section 3.1.2, the Jeans equation requires two boundary conditions (plus one more condition to fix the parameter $\bar{\sigma}_{\boldsymbol{v}}$ ). We choose $\rho^{\prime}(0)=0$ (cored solution) and $M_{\mathrm{CDM}}\left(r_{\mathrm{SIDM}}\right)=M_{\mathrm{SIDM}}\left(r_{\mathrm{SIDM}}\right)$. In this approach an additional boundary condition of equal density at $r_{\text {SIDM }}$ is assumed, $\rho_{\mathrm{CDM}}\left(r_{\mathrm{SIDM}}\right)=\rho_{\mathrm{SIDM}}\left(r_{\mathrm{SIDM}}\right)$.

The problem of this method is well-illustrated by Figures 3.17 and 3.18 where we present the results of the application of this procedure to one observed object and one simulated object. The first figure shows the observed data for the Milky-Way dwarf galaxy Carina. We applied the method described above three times, for the cross-sections 1, 3 and $100 \mathrm{~cm}^{2} / \mathrm{g}$. For this type of objects, the best measurement of the mass with the smallest uncertainty is at the half-light radius $r_{h}$. For the sake of the current experiment, we will assume that the cored solution in the inner part is preferred. We find the best fit cored 

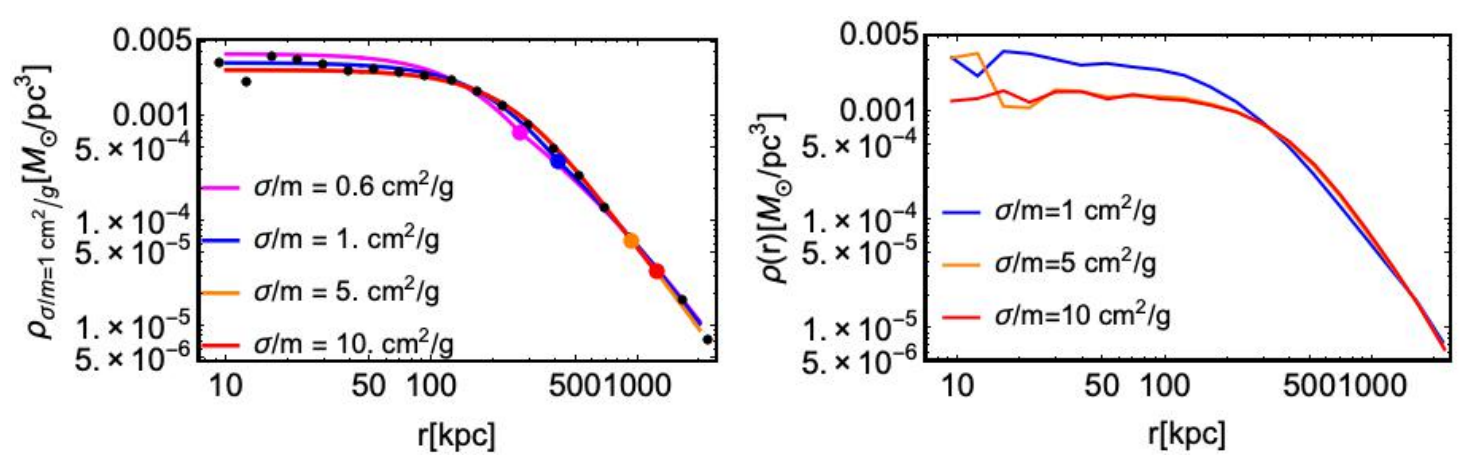

Figure 3.18: Left panel: The density profile of a simulated cluster with the self-interaction cross-section $1 \mathrm{~cm}^{2} / \mathrm{g}$ (black dots). Color lines are the best fits of that correspond to crosssections 1,5 and $10 \mathrm{~cm}^{2} / \mathrm{g}$. Right panel: Simulated density profiles of the same cluster for these cross-sections.

solution of the Jeans equation describing the inner density profile inside $r_{h}$. Then for each assumed cross-section we can define $r_{\text {SIDM }}$ using the density and velocity profiles of this best fit solution in the inner part. At this radius we impose the boundary conditions used in $[100,105,106,131,184]$, equal mass and density at $r_{\text {SIDM }}$. These conditions allow fixing the two parameters of the NFW profile for the outer part. Therefore, in this way we solve the opposite problem, as compared to what we did in the previous sections - reproduce the outer NFW assuming the core radius and the core density.

We see in Fig. 3.17 that all three NFW profiles obtained in this way are in agreement with the data. Moreover, the difference between the cross-sections 3 and $100 \mathrm{~cm}^{2} / \mathrm{g}$ is very small. We see that it would be difficult therefore to infer the value of the cross-section from the Carina dwarf, even if we know that the core exists. For the cross-sections $>1 \mathrm{~cm}^{2} / \mathrm{g}$ the dependence of the predicted outer NFW on the cross-section is too weak and therefore not observable. The situation for other dwarfs galaxies is similar. In [106] it is claimed that the sensitivity of the method to the cross-section improves a bit if one imposes the $c-M$ relation on the outer NFW profile. However, the scatter of the observed (and simulated) haloes in the $c-M$ relation can be up to an order of magnitude [186] and therefore we expect that if the scatter is taken into account the uncertainty in the constraining crosssection will remain large.

In the left panel of Fig. 3.18 we take the simulated data for a cluster-sized halo with the cross-section $1 \mathrm{~cm}^{2} / \mathrm{g}$. For this object we find the best fit profile for the cross-sections $1 \mathrm{~cm}^{2} / \mathrm{g}, 5 \mathrm{~cm}^{2} / \mathrm{g}$ and $10 \mathrm{~cm}^{2} / \mathrm{g}$ using the same method. Again, we see that profiles for these cross-sections fit simulation data equally well.

Hence, for both objects, we made fits using the method with "one collision per particle" and assuming different cross-sections. It is easy to see that we cannot distinguish the crosssection $1 \mathrm{~cm}^{2} / \mathrm{g}$ from larger cross-sections using these fits. 
The method to connect $r_{\text {SIDM }}$ with the cross-section $\sigma / m$ using "one collision per particle", described in the literature, does not allow to infer the value of the self-interaction cross-section from the observational data.

\subsubsection{Surface density and model-dependent constraints on SIDM}

In paper I [28] we did not have enough simulated data to compare with. Therefore, we described the SIDM model using an analytical model. To make this model more realistic, we introduced in the model unknown parameters describing systematic uncertainties in the model and tried to fix these parameters from the available simulations. Even if in the next Section 3.3 we will present an improvement of this method using more direct comparison between the data and simulations, we will review the methodology and the constraints from paper I [28] here. We will start introducing the main observable used in that paper - the DM surface density. The properties of this quantity allow to derive a constraint on SIDM using the whole ensemble of the observed objects.

\section{Surface density}

Let us remind the definition of the mean surface density

$$
\Sigma(r) \equiv \frac{M(r)}{\frac{4}{3} \pi r^{2}} \equiv\langle\rho\rangle_{r} r
$$

and

$$
M(r)=4 \pi \int_{0}^{r} r^{\prime 2} \rho\left(r^{\prime}\right) d r^{\prime}
$$

is the mass inside the radius $r$.

- For a cored profile:

$$
\begin{aligned}
& \Sigma(r) \simeq \text { const } \cdot r \quad \text { for } r<r_{\text {core }} \\
& \Sigma(r) \simeq \text { const } / r^{2} \cdot r=\text { const } / r, \quad \text { for } r>r_{\text {core }}
\end{aligned}
$$

Therefore, $\Sigma(r)$ is maximized at a radius close to the core radius.

- For the NFW profile

$$
\Sigma(r)=\frac{\text { const }}{r} r \approx \frac{3}{2} \rho_{s} r_{s}-2 \rho_{s} r, \quad r \ll r_{s}
$$

reaches its maximum for $r \rightarrow 0$, with $\Sigma(0)=1.15 \Sigma\left(0.1 r_{s}\right)=2.62 \Sigma\left(r_{s}\right)$. We will denote this maximal value as $\Sigma_{\max }$. 


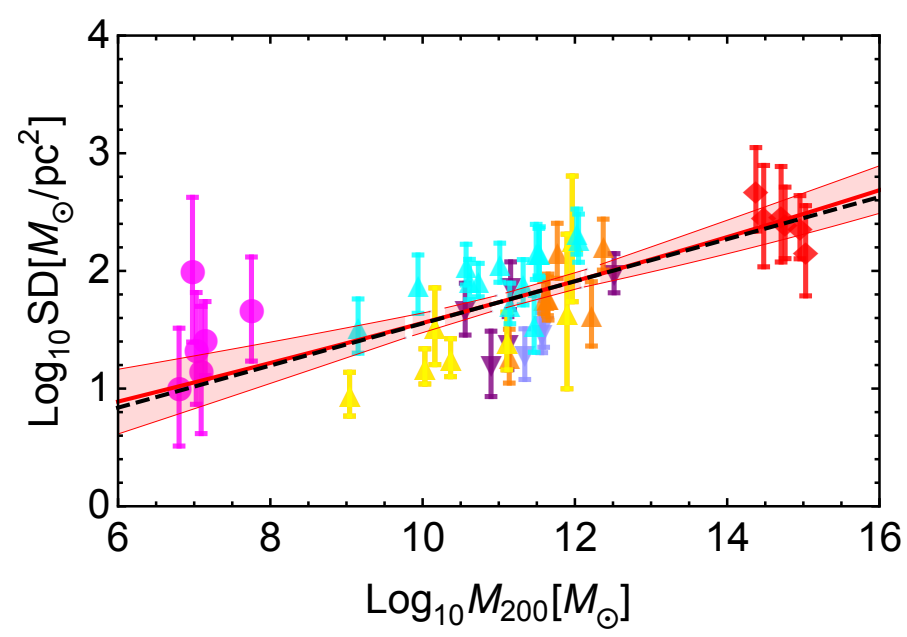

Dwarf

- LSB

$\checkmark$ GHASP

THINGS

LITTLETHINGS

SPARC

- Cluster

Figure 3.19: The surface density inside $r_{s}$ of the best fit NFW profile, as a function of $M_{200}$ (see 3.3.1). The data are fitted by $\Sigma \propto M_{200}^{n}$ as given in Eq. (3.28) (dashed line). The red solid line represents the best fit of the concentration-mass relation c.f. Eq. (3.36), the shaded region shows $2 \sigma$ variation of the slope and normalization. Taken from paper I [28].

It was originally argued in Refs. $[187,188]$ that $\Sigma_{\max }$ does not depend on the mass and the type of the galaxy (up to a certain scatter around the constant value) for objects from dSphs to elliptical galaxies. However, Refs. [189, 190] showed that this conclusion was based on a limited range of the halo masses. When taking into account all types of DM haloes, up to massive galaxy clusters, it becomes clear that $\Sigma_{\max }$ increases with $M_{200}$. This result has later been confirmed with larger data sets (see e.g. [191-193]) with a scaling relation given by [193]

$$
\Sigma_{\max } \propto M_{200}^{0.20 \pm 0.05} .
$$

The paper [190] provided a derivation of this scaling law within the CDM picture.

\section{$\Lambda$ CDM interpretation}

The scaling relation for the surface density as a function of $M_{200}$ finds a very natural interpretation in $\Lambda \mathrm{CDM}$ cosmology. For an NFW halo, we have

$$
M_{\mathrm{NFW}}(r)=4 \pi \rho_{s} r_{s}^{3}\left[\log \left(\frac{r_{s}+r}{r_{s}}\right)-\frac{r}{r+r_{s}}\right],
$$

and hence

$$
\Sigma_{\mathrm{NFW}}\left(r_{s}\right)=\frac{3}{2}(\log 4-1) \rho_{s} r_{s} \approx 0.579 \rho_{s} r_{s} .
$$

Fig. 3.19 presents the surface density for the objects in our sample, calculated using the NFW fits reported in the literature (see papers I and II [28, 29]). The error bars for the 
data points are calculated using uncorrelated $1 \sigma$ errors on the parameters of the NFW fits given in the corresponding papers. We then fit these data points with

$$
\Sigma_{\mathrm{NFW}}\left(r_{s}\right)=0.58_{-0.27}^{+0.50}\left(\frac{M_{200}}{M_{\odot}}\right)^{0.179 \pm 0.024} \mathrm{M}_{\odot} / \mathrm{pc}^{2} .
$$

It is instructive to re-express this relation in terms of $r_{200}$ and the halo concentration. $r_{200}$ is defined by the condition that the mean density $\langle\rho\rangle$ inside this radius is related to the critical density $\rho_{c}=3 H_{0}^{2} /(8 \pi G)$ by a factor of 200 . Therefore

$$
r_{200} \equiv\left(\frac{G}{100 H_{0}^{2}}\right)^{\frac{1}{3}} M_{200}^{1 / 3}=1.62 \cdot 10^{2}\left(\frac{M_{200}}{10^{12} h^{2} M_{\odot}}\right)^{\frac{1}{3}} \mathrm{kpc} .
$$

We remind that the halo concentration is

$$
c \equiv \frac{r_{200}}{r_{s}},
$$

This definition of the halo concentration is specific for the NFW profile. This implies that the NFW parameter $\rho_{s}$ is

$$
\rho_{s} \equiv \rho_{c} \delta=\rho_{c} \frac{200}{3} \frac{c^{3}}{\log (1+c)-c /(1+c)},
$$

which allows us to exchange the parameters $\left(\rho_{s}, r_{s}\right)$ for $\left(M_{200}, c\right)$.

The halo mass and the concentration are not independent. They are known to be related by a simple scaling law that is known from numerical simulations [186, 194-199], and is confirmed by observations [200-208]. Both, the slope and normalization of this scaling law differ in the literature. One of the more often used results is the one by Macciò et al. [186]

$$
c=8.3\left(\frac{M_{200}}{10^{12} h^{-1} M_{\odot}}\right)^{-0.104} .
$$

Of course, this relation is only an average and the concentration of a halo for a given mass has significant object-to-object scatter. We can use the data for the surface density inside $r_{s}$ as a function of mass to fix the concentration-mass relation independently. For this, we calculate the surface density for NFW profile inside the scale radius. We thus find

$$
\begin{aligned}
\Sigma_{\mathrm{NFW}}\left(r_{s}\right) & =\frac{3(\log 4-1)}{8 \pi}\left(\frac{100 H_{0}^{2}}{G}\right)^{\frac{2}{3}} M_{200}^{\frac{1}{3}} \frac{c^{2}}{\log (1+c)-c /(1+c)} \\
& =1.74 h^{2}\left(\frac{M_{200}}{10^{12} h^{2} M_{\odot}}\right)^{\frac{1}{3}} \frac{c^{2}}{\log (1+c)-c /(1+c)} \frac{M_{\odot}}{\mathrm{pc}^{2}}
\end{aligned}
$$



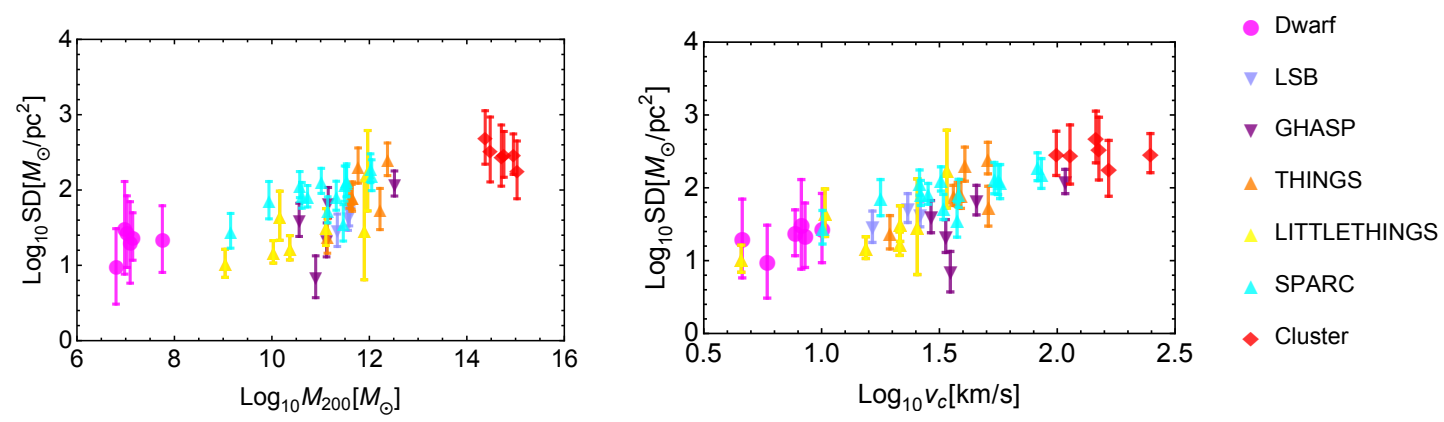

Figure 3.20: The dependence of the surface density at the experimental core radius $r_{\text {core }}$, as function of $M_{200}$ and the circular velocity $v_{c}$ at this radius, for the objects described in more detail in Section 3.3.1. Taken from paper I [28].

Then we assume a power law for the concentration-mass relation

$$
c=A\left(\frac{M_{200}}{10^{12} h^{-1} M_{\odot}}\right)^{\beta} .
$$

We substitute this into Eq. (3.34) and find parameters $A$ and $\beta$ fitting the data shown in Fig. 3.19. Finally,

$$
c=(10.8 \pm 0.6)\left(\frac{M_{200}}{10^{12} h^{-1} M_{\odot}}\right)^{-0.103 \pm 0.015} .
$$

In Fig. 3.19 we show the resulting surface density as the solid red line with the shaded red region indicating the uncertainty in the concentration-mass relation that we derived.

\subsubsection{DM surface density in SIDM halo: semi-analytic predictions}

The core radius $r_{\text {core }}$ is defined by Eq. (3.2.1) in a way that is relatively independent of which cored profile is used for the fit, and typically more robustly constrained observationally. The "observed" surface density at this core radius is then simply given by

$$
\Sigma_{c, \text { obs }}=\left\langle\rho_{\text {cored }}\right\rangle_{c} r_{\text {core }}
$$

where we note that $\left\langle\rho_{\text {cored }}\right\rangle_{c} \approx \rho_{\text {cored }}(0)=\rho_{\mathrm{NFW}}\left(r_{\text {core }}\right)$ because the DM density inside $r_{\text {core }}$ is almost a constant. We plot $\Sigma_{c \text {, obs }}$ in Fig. 3.20, for the same objects that we used in Fig. 3.19, as a function of both the virial mass and the circular velocity at the core radius.

We can obtain the theoretically expected surface density as

$$
\Sigma_{c, \text { theo }}=\langle\rho\rangle_{c} r_{\text {core }}=\kappa r_{\text {core }}\langle\rho\rangle_{\mathrm{SIDM}},
$$

where $\kappa$ is given by Eq. (3.14). As we argued in Section 3.2.7, $\kappa$ is difficult to determine 
observationally or directly from first principles. On the other hand it is a quantity that turns out to be tightly constrained by simulations, see Fig. 3.11. We can relate $r_{\text {SIDM }}$ and $r_{\text {core }}$,

$$
\rho_{\mathrm{NFW}}\left(r_{\text {core }}\right)=\rho_{\text {cored }}(0) \approx\langle\rho\rangle_{c}=\kappa\langle\rho\rangle_{\mathrm{SIDM}}=\kappa \frac{M_{\mathrm{NFW}}\left(r_{\mathrm{SIDM}}\right)}{(4 \pi / 3) r_{\mathrm{SIDM}}^{3}} .
$$

Solving this equation one can find $r_{\text {core }}\left(\kappa, r_{\text {SIDM }}\right)$ for given NFW parameters. So, the surface density inside the core radius can be written as a function of $\kappa$ and $r_{\mathrm{SIDM}}$,

$$
\Sigma_{c, \text { theo }}\left(\kappa, r_{\mathrm{SIDM}}\right)=\kappa r_{\text {core }}\left(\kappa, r_{\mathrm{SIDM}}\right) \frac{M_{\mathrm{NFW}}\left(r_{\mathrm{SIDM}}\right)}{(4 \pi / 3) r_{\mathrm{SIDM}}^{3}} .
$$

Moreover, using the approach discussed in Section 3.2.5 we can find the density profile of a SIDM halo at small radii for the given cross-section $\sigma / m$ if one knows $r_{\text {SIDM }} \equiv r_{M}$ and NFW parameters. So, the parameter $\kappa$ is not independent and can be found for fixed $r_{\text {SIDM }}$ and NFW parameters:

$$
\kappa=\kappa\left(\sigma / m, r_{\mathrm{SIDM}}\right)
$$

To predict the surface density $\Sigma_{c}$ for a given cross-section $\sigma / m$ we need to know only one quantity: $r_{\text {SIDM }}$.

The relation between $r_{\text {SIDM }}$ and the cross-section is shown in Fig. 3.14. As we already discussed in Section 3.2.6, it is quite difficult to build a theoretical model to predict it. So we can take this quantity from the SIDM simulations.

\subsubsection{Statistical constraints from the surface density data and semi- analytic predictions}

Let us now make a comparison of the results of the previous section with the estimates based on parametric profiles from the literature (see Appendix $C$ and Fig. 3.20). The results of this comparison for several cross-sections are given in Fig. 3.21.

For this we use an effective cross-section $\tilde{\sigma}=\sigma / \xi$ where $\xi$ is a number of collisions at $r_{\text {SIDM }}$ defined in Eq. (3.2) and represents the uncertainty in the relation between $r_{\text {SIDM }}$ and $\sigma$ discussed in the previous sections. Fig. 3.21 shows that for large enough $\tilde{\sigma} / m_{\chi}$ the two ensembles of haloes almost do not overlap and therefore such a model would be excluded by the data.

We can try to promote this qualitative picture to a more quantitative result using the likelihood ratio test [209]. We define the total likelihood as a product of normal distributions over each object ("data point") $i$,

$$
\mathcal{L}=\Pi_{i} N\left(f_{i} \mid \mu_{i}, \sigma_{i}\right)
$$



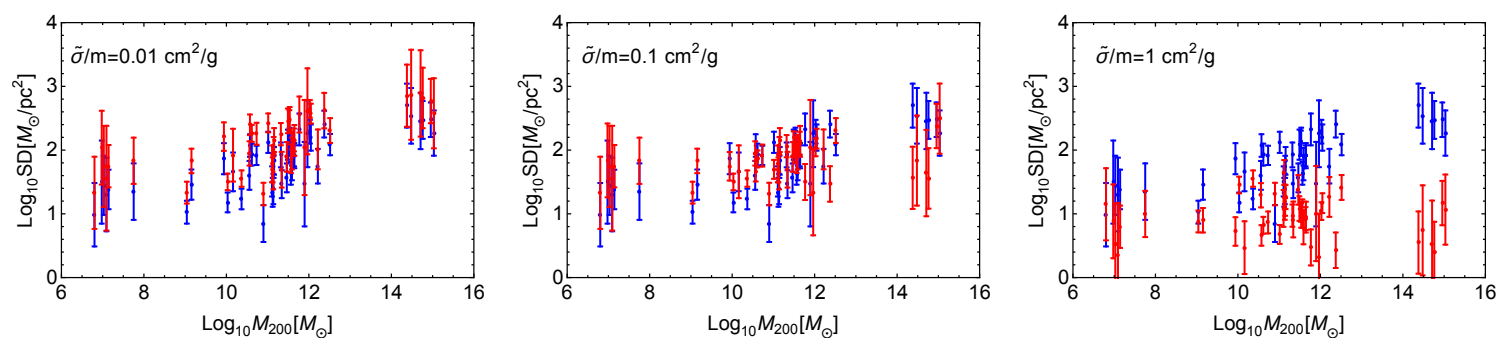

Figure 3.21: The surface density inside the core radius $r_{\text {core }}$, as function of the halo mass: observed values (from Fig. 3.20) - blue points, theoretical predictions for several crosssections from Eq. (3.38) - red points. Taken from paper I [28].

were $f_{i}$ is the logarithm of surface density inferred from the kinematical anlaysis (see Eq. (3.37)), $\sigma_{i}$ its variance, $\mu_{i}$ is the logarithm of the predicted surface density (see Eq. (3.38)) that depends on nuisance parameters $\left\{\alpha_{k}\right\}$. We add observational and theoretical errors in quadrature, $\sigma_{i}^{2}=\sigma_{i, \text { obs }}^{2}+\sigma_{i \text {,heo }}^{2}$. The errors in the "observed" surface density uncertainty, $\sigma_{i, \text { obs }}=\Delta \Sigma_{c \text {, obs }}$, are determined as shown in Fig. 3.20. In the "theory error" in this figure we include two contributions:

$$
\sigma_{i, \text { theo }}^{2}=\sigma_{i, \text { halo }}^{2}+\sigma_{t_{\text {age }}}^{2} \text {. }
$$

The main errors on $\sigma_{i \text {,halo }}$ are: a) from the variance in the core radius $r_{\text {core }}$, which is determined in the same way as for $\sigma_{i, \text { obs }}$ and affects predicted value of $\mu_{i}$ via Eq. (3.38), and b) from errors on $\rho_{s}$ and $r_{s}$ in each halo.

The value of $\kappa$ in Eq. (3.38) we extract from SIDM simulations of [99, 118, 124] with halo masses between $10^{10}$ and $10^{15} M_{\odot}$. We find that $\langle\kappa\rangle=3.9$ with a scatter of $\sigma_{\kappa}^{2} \equiv$ $\left\langle\kappa^{2}\right\rangle-\langle\kappa\rangle^{2}=1.4^{2}$, that is in a good agreement with the right panel of Fig. 3.11. Deriving constraints on the self-interaction cross-section we vary $\kappa$ freely within this range.

In Fig. 3.22 we show the full likelihood as a function of $\tilde{\sigma} / m_{\chi}$. The SIDM effect is represented by one non-negative degree of freedom. From this, we can read off an upper bound of

$$
\tilde{\sigma} / m_{\chi} \lesssim 0.12 \mathrm{~cm}^{2} / \mathrm{g}
$$

which corresponds to a 95\% C.L. limit. We have allowed $\kappa$ to vary up to its $2 \sigma$ upper bound.

To estimate possible difference between $\tilde{\sigma}$ and the physical $\sigma$ we apply the same analysis to 36 simulated haloes with the cross-section $1 \mathrm{~cm}^{2} / \mathrm{g}$ with masses from $5 \cdot 10^{9} M_{\odot}$ to $3 \cdot 10^{15} M_{\odot}$ from Refs. [99, 118]. In Fig. 3.23 we show the ratio of the reconstructed and true values of the cross-section. The best-fit value of this ratio is

$$
\xi=\frac{\left(\sigma / m_{\chi}\right)_{\operatorname{sim}}}{\tilde{\sigma} / m_{\chi}}=1.86 \pm 0.32
$$




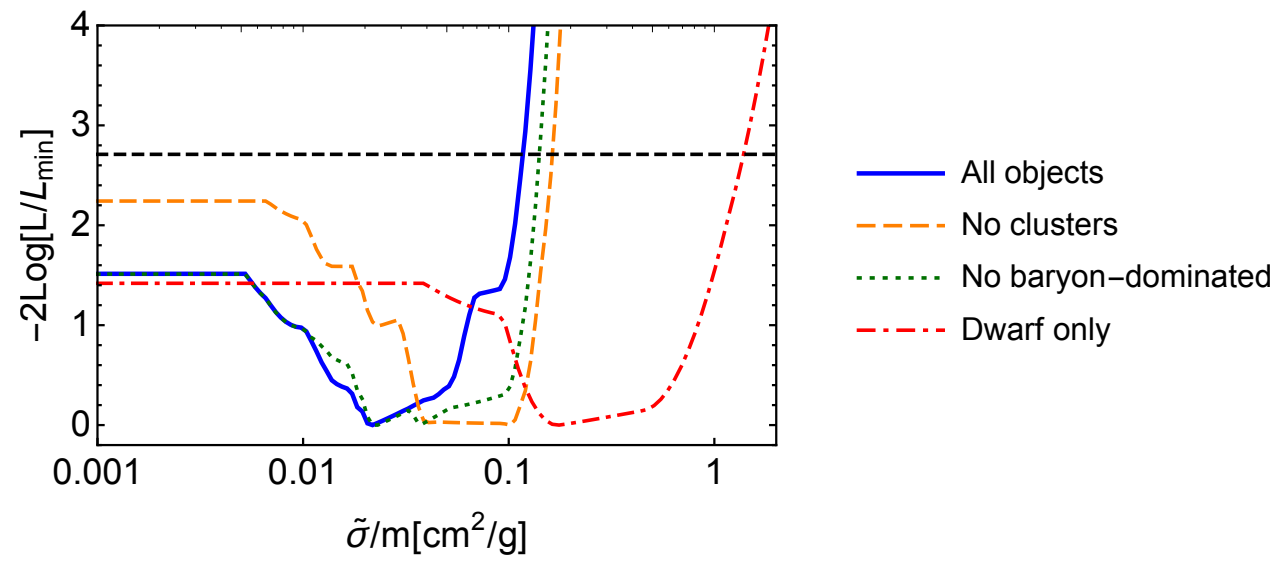

Figure 3.22: The log-likelihood ratio as function of the effective cross-section $\tilde{\sigma} / m_{\chi}$ (blue solid line). Orange dashed line - the result excluding clusters, green dotted line - excluding objects with baryon-dominated central part, red dot-dashed line - the result based on the dwarf galaxies only. Dashed horizontal black line - the log-likelihood ratio corresponding to the $95 \%$ CL limit. Taken from paper I [28].

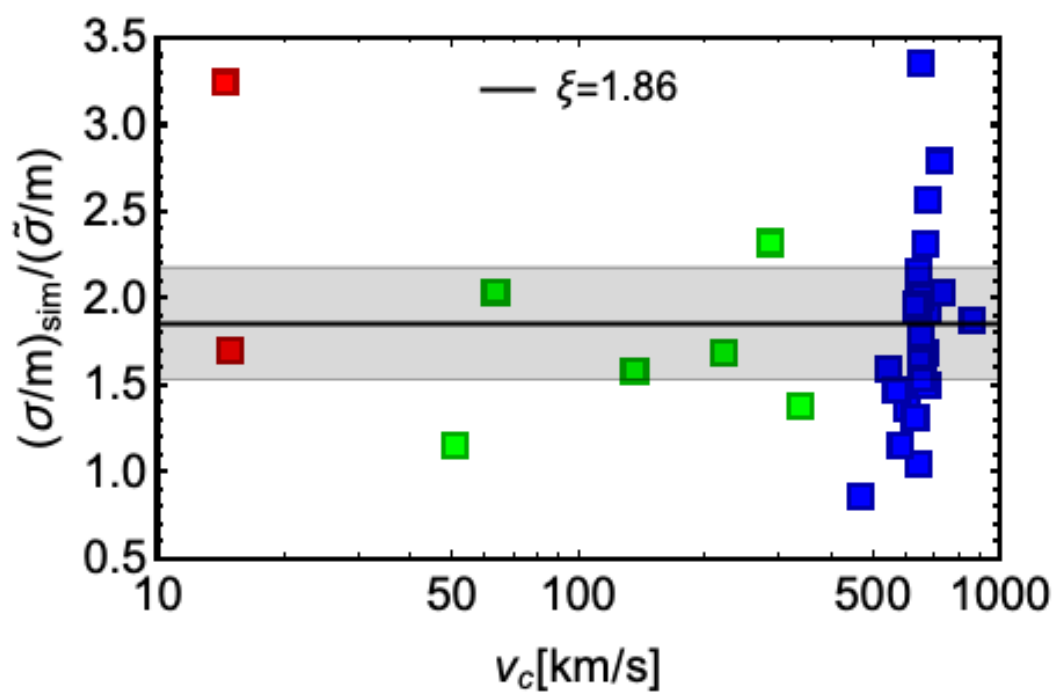

Figure 3.23: Parameter $\xi$ for the simulated haloes from [118] (green points), [99] (red points) and [124] (blue points).

Using this value we obtain a limit of

$$
\sigma / m_{\chi} \lesssim 0.3 \mathrm{~cm}^{2} / \mathrm{g}
$$

for the physical self-interaction cross-section. Of course, this result is the subject both to experimental uncertainties (as we used the fits to parametric profiles to describe observational data) and to theoretical uncertainties discussed above (that we tried to fix using Eq. 3.45). In the next section, we will discuss how we can improve this result. 

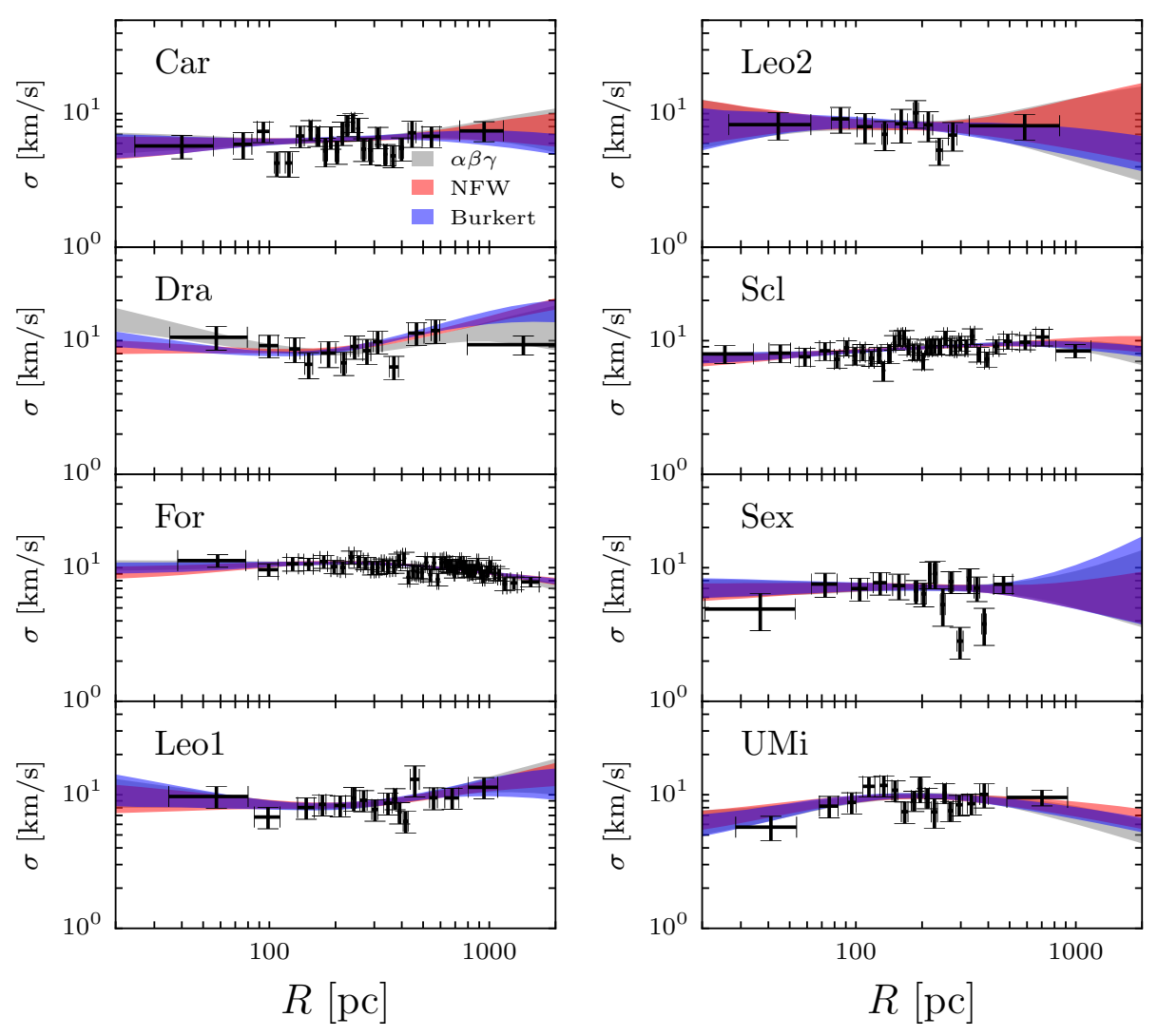

Figure 3.24: The observed velocity dispersion of classical dwarf spheroidal galaxies as a function of radius. Black points - observational data, and the red (blue, gray) line are fits for NFW( [185]) (Burkert [185], generalized NFW) profile [214]). The width of the lines represents $1 \sigma$ uncertainty of best fit parameters. Taken from paper I [28].

\subsection{Direct comparison of simulations with observations}

In this section, we will try to compare SIDM simulations with observations more directly. Namely, we will avoid any analytic model of SIDM and will also derive the observed surface density from the data without using parametric profiles (like NFW, etc). To address this more ambitious task we will require more simulations, in particular (as large as possible) samples of SIDM simulations of haloes of all sizes. We will also use simulations with baryons to model possible baryonic effects on the relevant observables. The following simulations were used in this section: dwarf galaxies [210], APOSTLE simulations [86, 211], Aquarius simulations [95, 212], and BAHAMAS simulations [213].

\subsubsection{Available observational data}



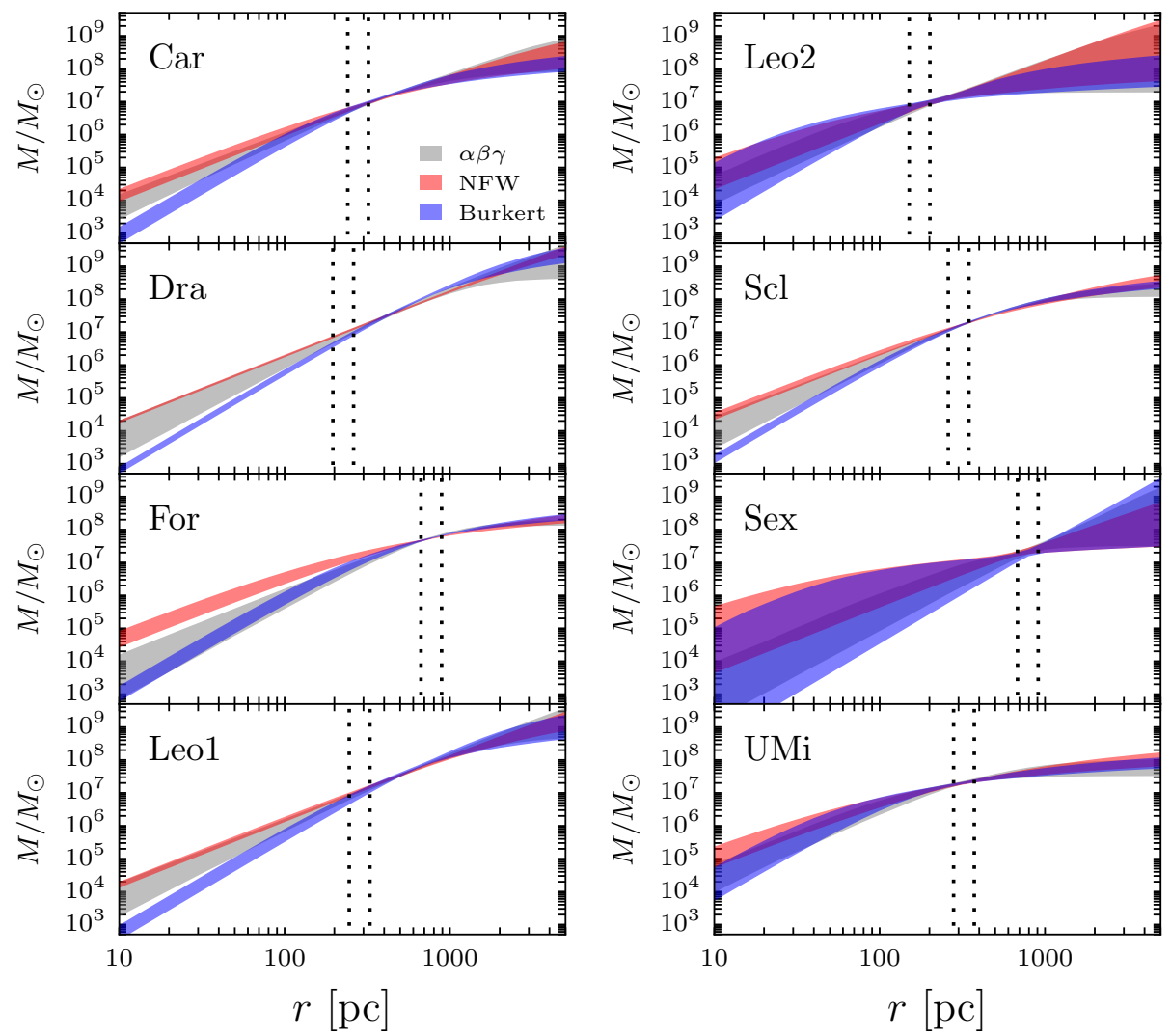

Figure 3.25: The data from Fig. 3.24 in terms of the enclosed mass. Dotted vertical lines - half-light radius $r_{h}$ with its uncertainty. Taken from paper I [28].

Dwarf Spheroidal galaxies. Dwarf Spheroidals (dSphs) are satellites of the Milky Waysize galaxies. They are observed in the Milky Way and in Andromeda. dSphs have the highest known mass-to-light ratio. They are DM dominated even in the central parts. These properties make dwarf spheroidals very special for studying DM properties. The socalled "classical dSphs" are known for a long time and well studied. In these objects, many hundreds of stars are observed. Therefore, for these objects, there are enough statistics to produce velocity dispersion in a significant number of radial bins (see Fig. 3.24).

The main observable in $\mathrm{dSphs}$ is the velocities of stars. The dispersion of velocities is used to reconstruct the gravitational potential and the mass enclosed in a given radius. The main uncertainty of this method is related to the fact that often it is possible to measure only the projections of the star velocities along the line of sight and not the full 3D velocities. The corresponding component of the velocity dispersion $\sigma_{\operatorname{los}}$ is not sufficient to reconstruct fully the enclosed mass. This uncertainty can be minimized when the analysis is applied to the mass inside the half-light radius $r_{h}$ [215-217], as illustrated in Fig. 3.25. In Fig. 3.24 we present the stars velocity dispersion as a function of radius. The reconstructed mass profile is shown in Fig. 3.25. 


\begin{tabular}{|l|c|c|c|c|c|}
\hline Object & Ref. & $r_{h}, \mathrm{pc}$ & $\sigma, \mathrm{km} / \mathrm{s}$ & $M\left(r_{h}\right), \mathrm{M}_{\odot}$ & $\Sigma\left(r_{h}\right), \mathrm{M}_{\odot} / \mathrm{pc}^{2}$ \\
\hline Sextans & {$[49]$} & 630 & 6.6 & $1.6 \times 10^{7}$ & 9.6 \\
Fornax & {$[49]$} & 400 & 10.5 & $2.6 \times 10^{7}$ & 38.3 \\
Leo I & {$[49]$} & 330 & 8.8 & $1.5 \times 10^{7}$ & 32.6 \\
Ursa Minor & {$[49]$} & 300 & 10.3 & $1.8 \times 10^{7}$ & 49.1 \\
Carina & {$[49]$} & 290 & 6.8 & $7.8 \times 10^{6}$ & 22.1 \\
Draco & {$[49]$} & 221 & 9.5 & $1.2 \times 10^{7}$ & 56.7 \\
Sculptor & {$[49]$} & 160 & 10.1 & $9.5 \times 10^{6}$ & 88.5 \\
Leo II & {$[49]$} & 185 & 6.8 & $4.9 \times 10^{6}$ & 34.7 \\
\hline
\end{tabular}

Table 3.2: The parameters for classical dSphs [49].

Recently, many more dwarf spheroidals were discovered by SDSS and DES surveys [218, 219]. For many of these objects high-resolution spectroscopic observations are missing and it is difficult to do reliable mass measurements.

As we described, the best mass measurement is at the half-light radius, $r_{h}$. In Table 3.2 we present the data for the half-light radii, velocity dispersions, masses at $r_{h}$ and surface densities at $r_{h}$ for dSphs.

The mass inside the half-light radius $r_{h}$ can be estimated as [36]:

$$
M\left(r_{h}\right)=\frac{2.5 \sigma^{2} r_{h}}{G_{N}}=5.81 \times 10^{2} \mathrm{M}_{\odot}\left(\frac{\sigma}{\mathrm{km} / \mathrm{s}}\right)^{2}\left(\frac{r_{h}}{\mathrm{pc}}\right),
$$

and the surface density is

$$
\Sigma\left(r_{h}\right) \equiv \frac{3 M\left(r_{h}\right)}{4 \pi r_{h}^{2}} \simeq 139 \mathrm{M}_{\odot} / \mathrm{pc}^{2}\left(\frac{\sigma}{\mathrm{km} / \mathrm{s}}\right)^{2}\left(\frac{r_{h}}{\mathrm{pc}}\right)^{-1}
$$

Spiral galaxies. The main observable in spiral galaxies is rotation velocities of stars and neutral hydrogen. For these objects, the existence of the observable disk allows measuring 3D velocities, at least for some of the objects (see below). The main uncertainty in spiral galaxies is due to the modeling of baryonic contribution to the total mass, as in their central parts these objects are dominated by baryons. As a result, the DM profile can often be fitted (almost) equally well with NFW and cored profiles, see e.g. the rotation curves displayed in Ref. [220]. The best measurement of the DM profile is derived from the flat part of the rotation curve, where DM dominates over baryons.

If the disk is perpendicular to the line of sight we cannot measure the rotation velocity. In the opposite limit when the disk of the galaxy is parallel to the line of sight we cannot say what is the position in the galaxy of each observed star. Therefore, the best measurements are obtained for galaxies with some intermediate inclination.

Another source of uncertainty is the distance to a galaxy. If the galaxy contains some standard candles (like supernova Ia or Red-giant branch) we can measure the distance to 

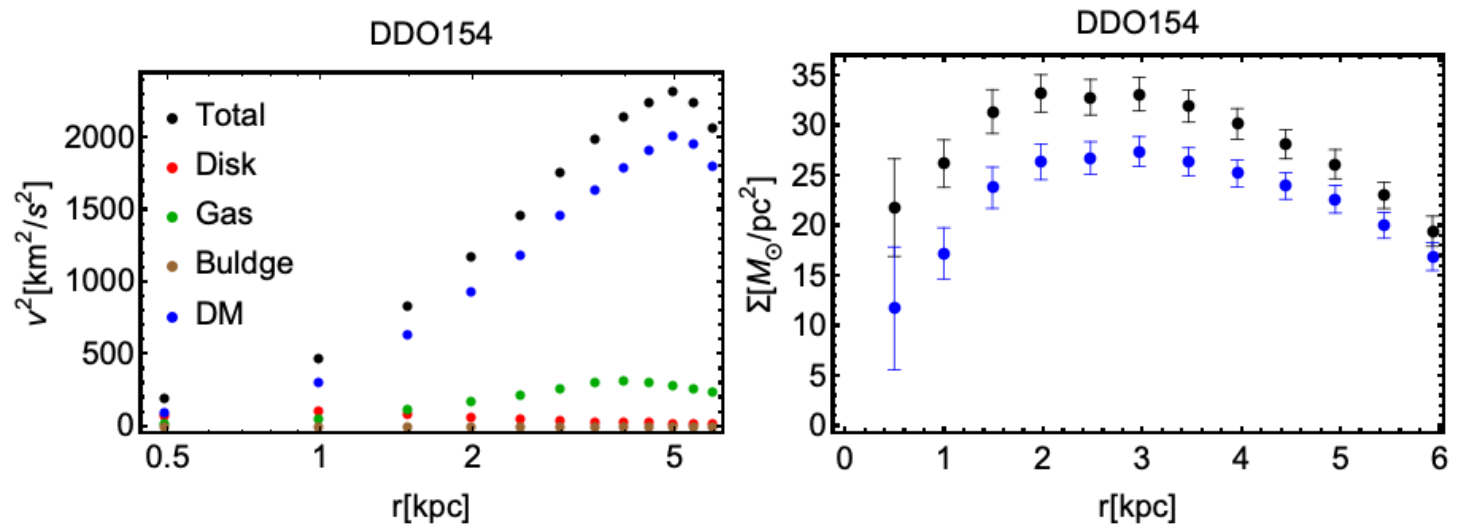

Figure 3.26: An example of a SPARC object. Left panel: Square of rotational velocities of different components. Right panel: Total surface density (black points) and DM only surface density (blue points).
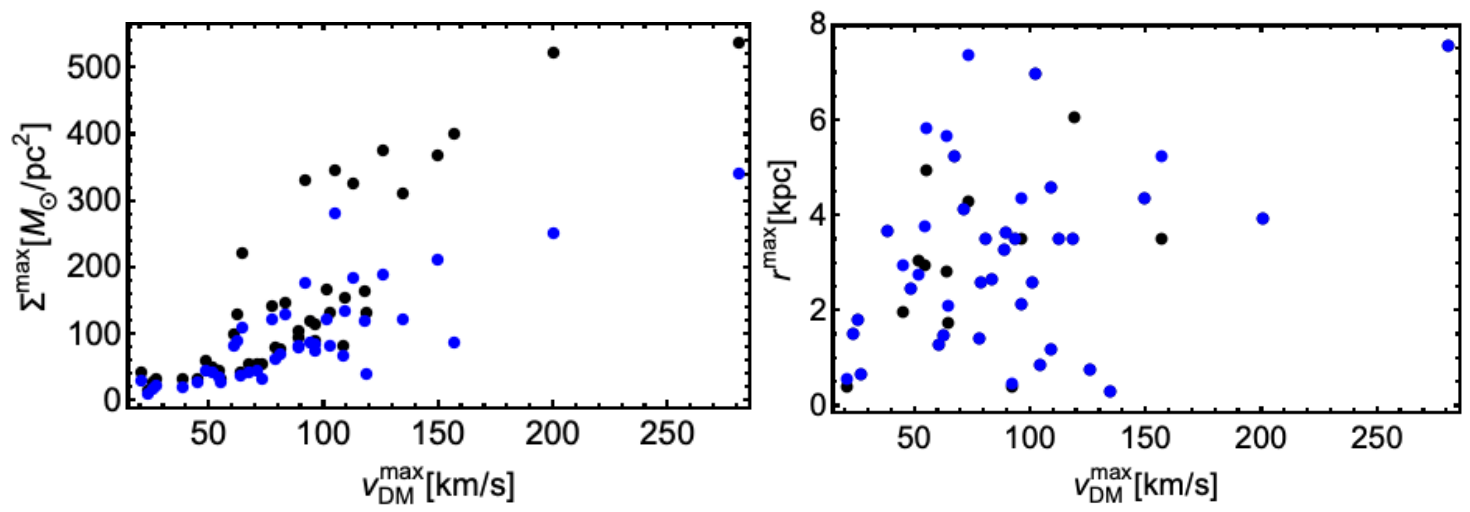

Figure 3.27: Left panel: The maximal total surface density (black points) and the maximal DM only surface density (blue points) versus the maximal rotational velocity. Right panel: Radii of the maximal surface density for the total mass (black points) and DM only (blue points) versus the maximal rotational velocity.

this galaxy with high precision. But for most of the galaxies, the main method to measure distances is the Hubble law. It is less accurate because of the peculiar velocities of each object, which we do not know exactly.

Most of the galaxies are baryon-dominated in the central part, so it is very important to know the mass-to-light ratio to measure the DM density. We took spiral galaxies from the SPARC catalogue [221] with 175 objects that also provided us with models of baryons based on the method described in [222]. Our selection criteria are: uncertainty of the distance measurement less than $15 \%$, the galaxy disk inclination more than $30^{\circ}$ and the quality flag is equal to 1 or 2 (which means the best objects, see details in [221]). After applying these cuts we left with 83 objects. These objects can be divided into 3 different groups: 
1. Objects with anomalies, e.g. contribution of baryons is larger than the mass from the total rotational velocity, there is no flat part in the rotational velocity (12 haloes);

2. Too small amount of data points (10 haloes);

3. Good objects (61 haloes).

An example of an object from the third group is given in Fig. 3.26. Using objects from the third group we show the maximum of the surface density and radius of the maximum of the surface density as a function of the maximal rotation velocity, see Fig. 3.27. We see that both the total and DM only maximal surface densities have a regular dependence on the rotational velocity, while radii of the maximum are spread randomly within the region of the plot.

Galaxy clusters. In clusters of galaxies, there are many tracers of gravitational potential and mass. These include dynamics of galaxies in the cluster, the temperature distribution of hot X-ray gas, strong and weak gravitational lensing. At large distances, the DM distribution in clusters is well described by the NFW profile. In the literature it was claimed [177] that in some clusters there is an evidence for the presence of cores in the inner DM density profile. However, clusters are typically baryon-dominated in the center. A significant contribution to the central mass of a cluster is given by the brightest central galaxy (that is usually also the most massive) and gas around its center. Therefore, the central DM density profile is very difficult to measure and the claims of cores are disputed, see e.g. the discussion in Ref. [223].

Here we try to take a more model-independent approach. We analyze a sample from 52 massive clusters from the Canadian Cluster Comparison Project (CCCP) [224] with redshifts $0.15<z<0.55$. In this sample to select the most massive clusters we adopt a constraint on the gas temperature $T_{X}>5 \mathrm{keV}$. We rejected 3 clusters (Abell 115, Abell 223 and Abell 1758) due to the fact that these objects experience a merger.

Weak lensing measures the 2-dimensional surface density (projected mass) that is given by

$$
\Sigma_{2 D}(R)=\frac{M_{2 D}(R)}{\pi R^{2}},
$$

where $M_{2 D}(R)$ is the mass inside a cylinder with radius $R$. For each cluster we take from the observational data three data points: $M_{2 D}(100 \mathrm{kpc}), M_{2 D}(200 \mathrm{kpc})$ and $M_{2 D}(300 \mathrm{kpc})$. We illustrate this quantity for CCCP clusters in Fig. 3.28.

\subsubsection{SIDM in simulations}

In this section, we introduce several additional suites of simulations of the ensembles of objects of every size (clusters of galaxies, spiral galaxies, dwarf galaxies) that we need for 


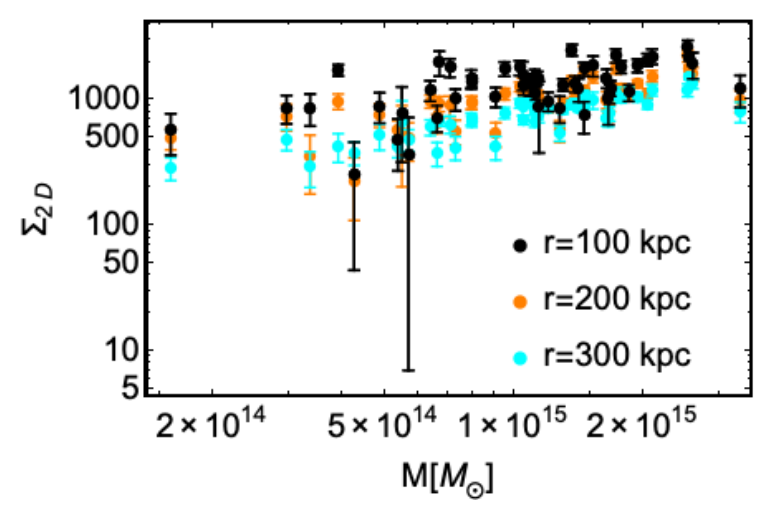

Figure 3.28: The 2-dimensional surface density for clusters from CCCP at 3 radii.

the comparison with observations. We study the dependence of the surface density on the radius for CDM and SIDM as well as effects of baryons on this dependence. Also, we analyze the difference in the behavior of the DM only and the total mass surface densities. For the case of SIDM, we study the dependence of the maximum of the surface density on the halo mass and concentration. This data will be used for the final comparison between simulations and observations that we will perform at the end of this Section.

\section{Description of the simulations}

Dwarf galaxies. We use eight (m10b, c, d, e, f, h, k, and $\mathrm{m}$ ) zoom-in simulations for CDM and SIDM cosmology with the cross-section $1 \mathrm{~cm}^{2} / \mathrm{g}$, that were described in the paper [210]. The softening radius is equal to $35 \mathrm{pc}$ and DM masses $2500 M_{\odot}$.

APOSTLE simulations. APOSTLE (A Project Of Simulating The Local Environment) simulation is a suite of cosmological hydrodynamic simulations of 12 simulations with the cube $100^{3} \mathrm{Mpc}^{3}$ with $1620^{3}$ particles in WMAP-7 cosmology that match the Local Group of Galaxies [86, 211]. The simulations for two of these volumes were rerun for the case of self-interacting Dark Matter. The SIDM version of these simulations was kindly provided by Andrew Robertson.

Initially we had 112 haloes with masses from $2.7 \cdot 10^{9} M_{\odot}$ to $1.5 \cdot 10^{12} M_{\odot}$. We have found that for small haloes resolution of the simulation is not enough to resolve the effects of SIDM. Therefore, we have selected only haloes with masses greater than $10^{10} M_{\odot}$ and $r_{M}$ (see 3.2.2) is greater than two trust radii (i.e. the radius where we can trust simulations). After this selection, we left with 48 haloes.

Aquarius simulations. The Aquarius simulation [95, 212] is a simulation of a MilkyWay sized DM haloes. The simulation cube of the size $100 h^{-1} \mathrm{Mpc}$ with the cosmological parameters $\Omega_{m}=0.25, \Omega_{b}=0.045, \Omega_{\Lambda}=0.75, \sigma_{8}=0.9, n_{s}=1, H_{0}=73 \mathrm{~km} / \mathrm{Mpc} / \mathrm{s}$. The particle mass depends on the resolution and for our analysis we use the resolution 2 
simulations (see Ref. [212]) with the mass $m_{p} \simeq 10^{4} M_{\odot}$ and softening $\epsilon \simeq 66$ pc. We select 5 of 6 haloes (A, B, C, D, and E), rejecting halo F due to a very non-trivial assembly history [225].

BAHAMAS simulations. BAHAMAS simulations (BAryons and HAloes of MAssive Systems) [213] are simulations of the local environment with a size of the box $400 \mathrm{Mpc} / \mathrm{h}$. There are $1024^{3} \mathrm{DM}$ particles with masses $\approx 5.5 \times 10^{9} \mathrm{~h}^{-1} M_{\odot}$ for a Planck cosmology. The SIDM run of this simulation is described in [226]. Simulations with baryons with masses of $3.85 \times 10^{9} h^{-1} M_{\odot}$ is discribed in details in [213].

To compare properties of CDM and SIDM haloes we have selected $10^{4}$ most massive friend-of-friends groups. From these groups, we found the most massive haloes with a mass greater than $10^{14} M_{\odot}$ and applied the virialization criteria (see Ref. [213]). Next we performed a matching procedure using positions of the center of gravitational potential with a matching condition $\left|\boldsymbol{r}_{\mathrm{SIDM}}^{\text {center }}-\boldsymbol{r}_{\mathrm{CDM}}^{\text {center }}\right|<0.5 \mathrm{Mpc}$. For some SIDM haloes, there was no CDM partner and we have rejected such haloes. After the matching procedure, we ended up with 159 haloes.

For each SIDM halo with the cross-section $\sigma / m=1 \mathrm{~cm}^{2} / \mathrm{g}$ we have calculated the radius of equal masses, $r_{M}$, between SIDM and CDM haloes. We have not found this radius for 13 haloes, mostly for the smallest haloes. For these haloes, the DM density and the velocity dispersion profiles are very close to $\mathrm{CDM}$ ones at radii larger than the trust radius.

\section{Surface density in DM-only simulations}

The surface density as a function of radius for different DM-only simulation is shown in Fig. 3.29. The difference between the CDM and SIDM models becomes apparent at small radii: for the SIDM model the surface density goes to zero, while for CDM it grows. Such behavior results in the existence of the maximal surface density in the case of SIDM.

SIDM can be distinguished from CDM by the existence of the maximal surface density.

The maximal surface density for SIDM simulation with the cross-section $1 \mathrm{~cm}^{2} / \mathrm{g}$ as a function of virial mass of objects is shown in Fig. 3.30. We clearly see the scaling law that we expected for the surface density.

\section{Effects of baryons}

Until now we have discussed DM only simulations. Now we want to answer the following question: how will this picture be changed by baryons? In the left panel of Fig. 3.31 we 

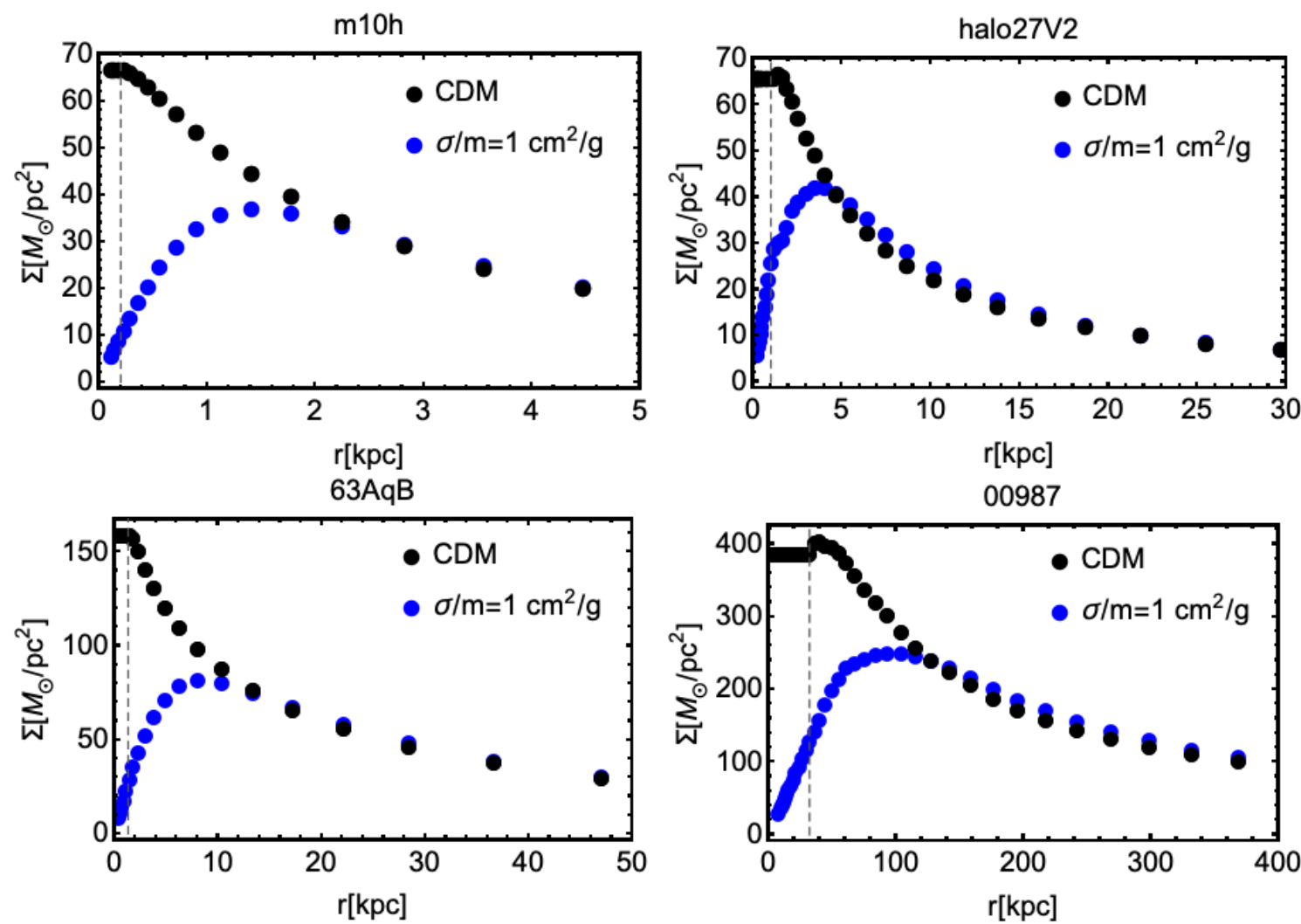

Figure 3.29: Examples of the surface density versus radius for DM-only simulations for CDM and SIDM cosmologies. A dwarf galaxy with $M_{200}=9.4 \cdot 10^{9} M_{\odot}$ - upper left, an object with $M_{200}=4.5 \cdot 10^{10} M_{\odot}$ from APOSTLE - upper right, an Aquarius halo (Aq.B, $\left.M_{200}=8.2 \cdot 10^{11} M_{\odot}\right)-$ bottom left, and a galaxy cluster from BAHAMAS $\left(M_{200}=\right.$ $\left.1.5 \cdot 10^{14} M_{\odot}\right)$ - bottom right.

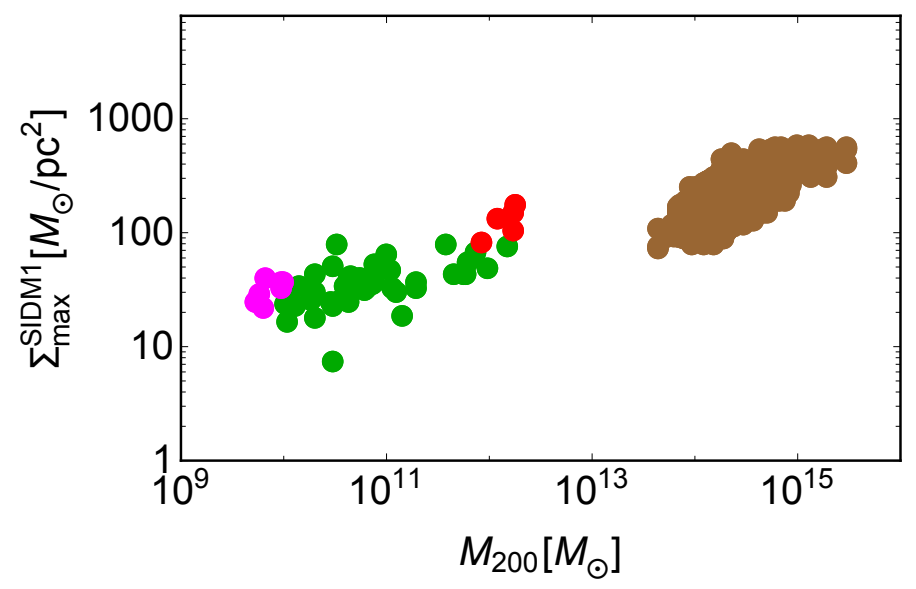

Figure 3.30: The maximum surface density versus virial mass for DM-only simulations: dwarf galaxies (pink), APOSTLE (green), Aquarius (red), and BAHAMAS (brown). 

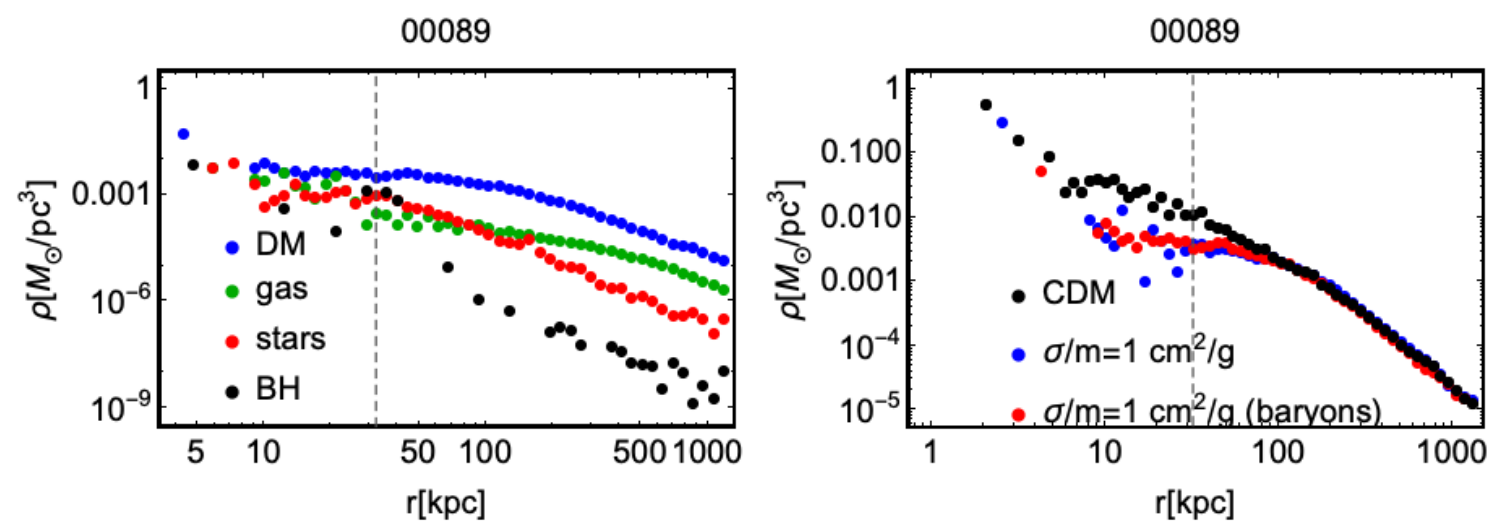

Figure 3.31: Density profiles for a halo from the BAHAMAS simulation for DM only and baryon components (left) and for the DM components for various cosmology.
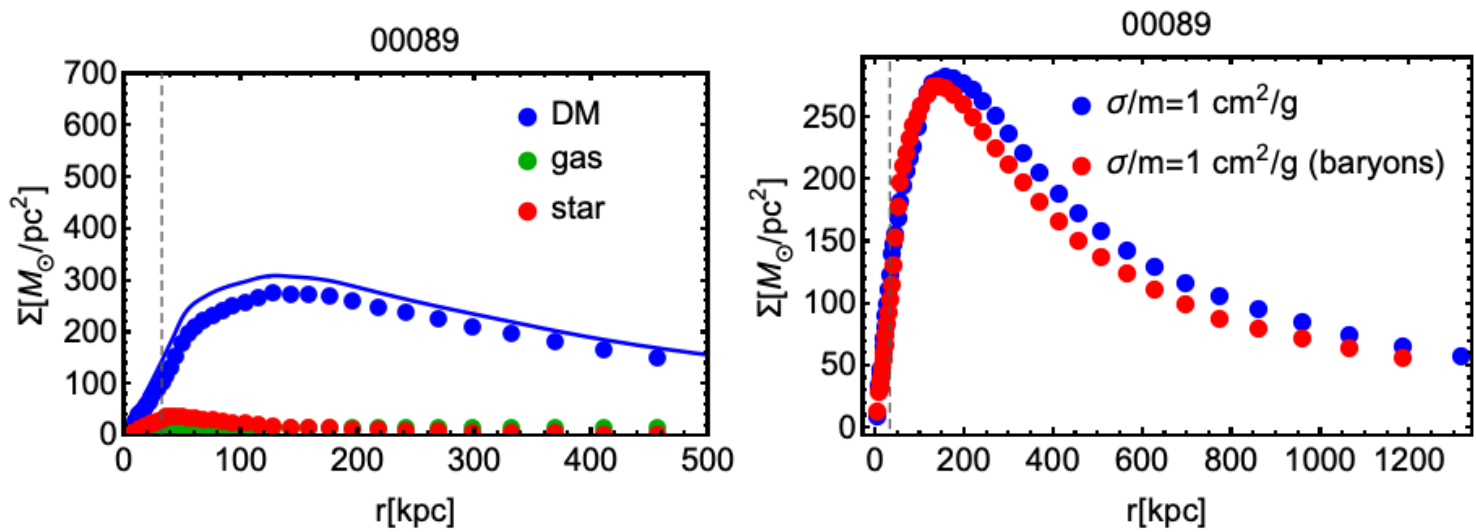

Figure 3.32: The surface density versus radius for DM, gas and stars (left) and for DM only components for simulations with and without baryons.

show the density profiles of the different components of a halo from BAHAMAS with baryons for the cross-section $\sigma / m=1 \mathrm{~cm}^{2} / \mathrm{g}$. As we see, the DM component dominates for all simulated radii, so we do not expect a large influence of baryons. This can be seen in Fig. 3.31 (right panel).

In Fig. 3.32 we show a comparison of the surface density for the SIDM halo with and without baryons. We see that the surface density profiles for the DM only in the simulations with and without baryons are very close to each other. The maximal surface density for different cases is shown in Fig. 3.33. We see that the DM surface density in SIDM simulations in the presence of baryons has the same power law and scatter as in the case without baryons and can be distinguished from the CDM case.

What is even more surprising, the surface density calculated using the total mass in simulations with baryons has the same behavior. In Fig. 3.34 we show an example of the total surface density for CDM and SIDM models with baryons. As we expected, the influence of baryons is quite mild. In Fig. 3.35 we compare the total surface density in 


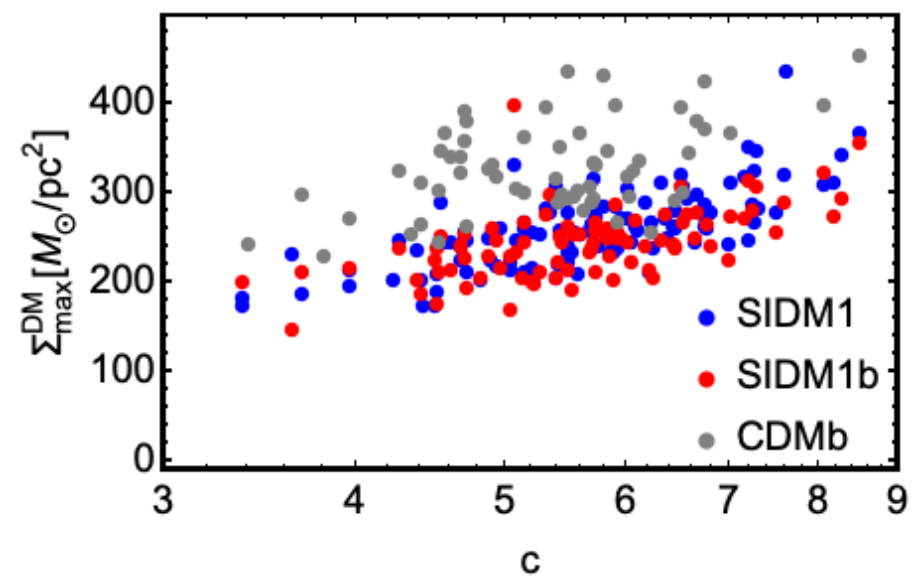

Figure 3.33: The maximal surface density for DM in simulations without baryons (blue points), DM in simulations with baryons (red points) and for CDM in the simulations with baryons (gray points). The self-interaction cross-section is $1 \mathrm{~cm}^{2} / \mathrm{g}$.

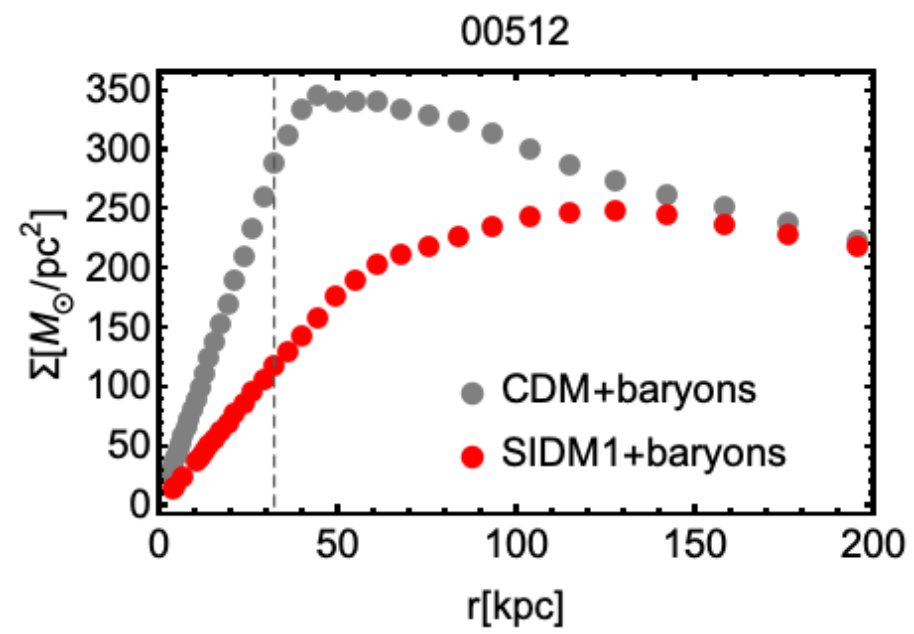

Figure 3.34: The total surface density for haloes with baryons from the BAHAMAS simulation for CMD (gray) and SIDM (red).

SIDM and CDM simulations with baryons. We can see that the average value for the maximal surface density is larger for CDM. Also, one can notice that the radius at which the surface density reaches its maximum for $\mathrm{CDM}$ is very close to the trust radius and it is a factor of few smaller than for SIDM (about 50 and $100 \mathrm{kpc}$ respectively).

We can distinguish between SIDM and CDM using the total surface density of the objects! 

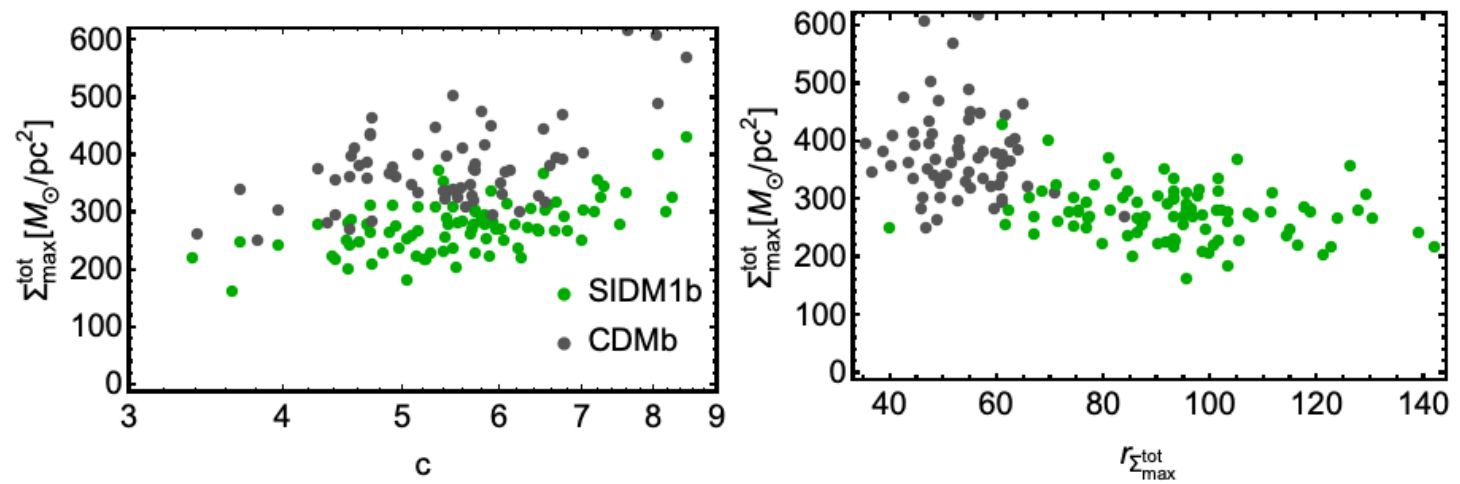

Figure 3.35: Left panel: The maximal surface density versus concentration for the total mass for CDM (gray) and SIDM (green). Right panel: The maximal surface density versus the radius at the maximal surface density for the total mass for CDM (gray) and SIDM (green).

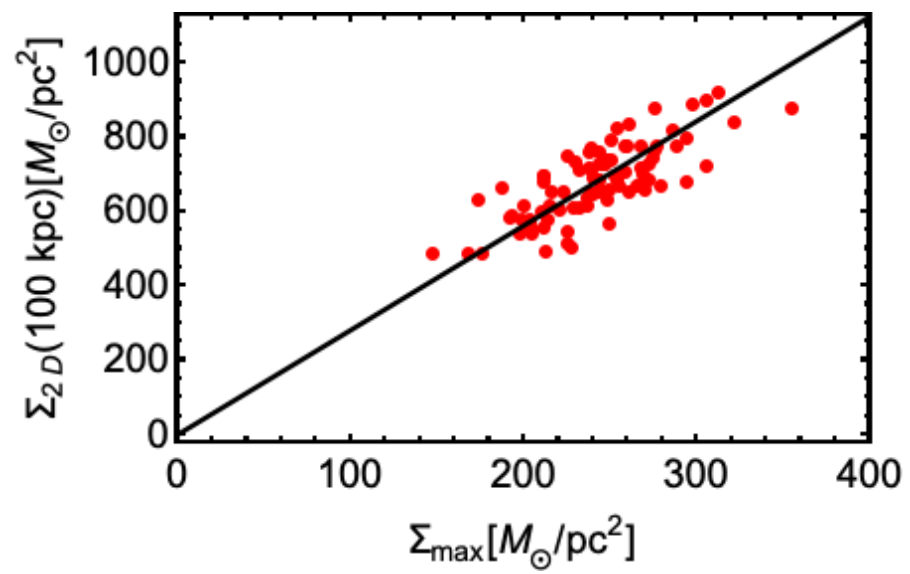

Figure 3.36: The maximal surface density versus the 2D surface density at the radius 100 kpc for BAHAMAS simulated clusters with baryons. The black line is a best fit for the linear function $y=a x$ with the slope $a \approx 2.8$.

\subsubsection{Comparison of the surface density between data and simula- tions}

In this section, we finalized our analysis and compare the observational data with the SIDM simulations for the cross-section $1 \mathrm{~cm}^{2} / \mathrm{g}$. As it was announced, we do it in a maximally model-independent way. As a quantity that allows working with the whole ensemble of haloes we still use the surface density. We use the total mass surface density rather than the DM-only surface density. Our results therefore are not dependant on the baryonic contribution. In simulations of galaxies and dwarfs, we take the maximum of the surface density for all radii. On the observational side, we observe a clear maximum of the total mass surface density only in the spiral galaxies. For the dwarf galaxies, we 


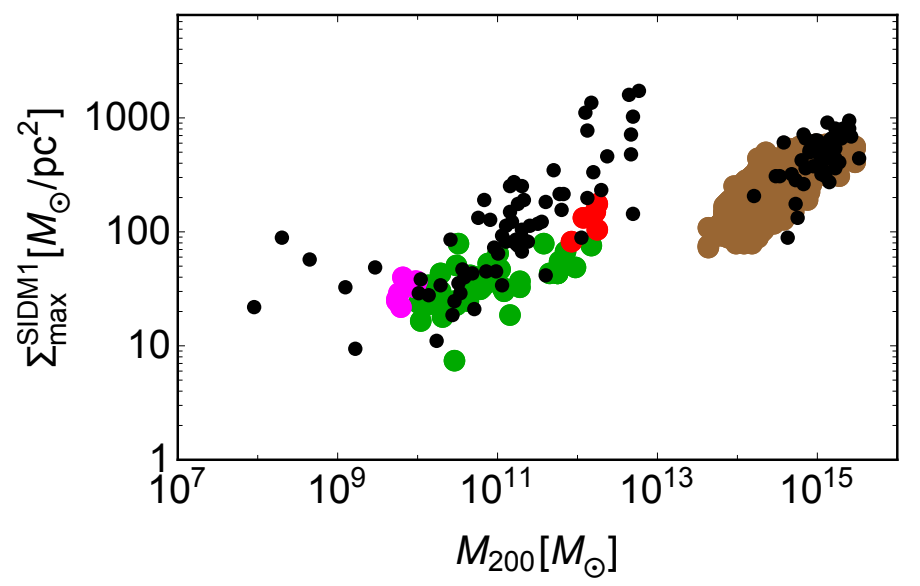

Figure 3.37: The maximal surface density versus $M_{200}$ for observed haloes (black) and simulated haloes: dwarf galaxies (magenta) [210], APOSTLE (green) [86], Aquarius (red) $[95,212]$, BAHAMAS simulations (brown) [213]. For clusters we calculate the $2 \mathrm{D}$ surface density and then normalize it by a factor of $a \approx 2.8$ (see text for details).

use only the most reliable measurement of the surface density inside the half-light radius, see Table 3.2. We use this value as a lower bound for the maximum surface density in dwarf galaxies. For clusters of galaxies, the maximum of the surface density among the available data points is reached at the smallest measured radius $-100 \mathrm{kpc}$. Therefore, we use this value as a lower bound for the maximal value of the surface density. This is consistent with Fig. $3.35-$ for $1 \mathrm{~cm}^{2} / \mathrm{g}$ and for objects of this size the maximum of the surface density is indeed located around $100 \mathrm{kpc}$. As discussed before, the measured quantity for the galaxy clusters is not the $3 \mathrm{D}$ surface density, calculated using the mass enclosed in a sphere, but a projected 2D surface density, that is related to the mass in a cylinder along the line of sight (see Fig. 3.36). To present this observational data on the same plot with the data from the rest of the objects we have checked in the simulations that $2 \mathrm{D}$ and $3 \mathrm{D}$ surface densities are in fact quite tightly related (see Fig. 3.36) - they are simply proportional with a coefficient 2.8 and very modest scatter around this relation. We use this one constant to re-scale the observational data for the galaxy clusters and add them to the final plot 3.37. This plot demonstrates that SIDM with the cross-section $\sigma / m=1 \mathrm{~cm}^{2} / \mathrm{g}$ predicts surface densities that are systematically a bit lower than the data. We would like to stress that this result is obtained using the total mass rather than the DMonly mass; direct experimental data rather than parametric modelings of the haloes and numerical simulations (with baryons) instead of approximate analytic models. This makes this result very robust and conservative. More work, both on simulation and observation sides, can improve this constraint. 


\subsection{Conclusions}

In this section, we have reviewed the constraints on the cross-section of the self-interacting Dark Matter obtained from the bounds on DM density cores in observed objects of different sizes (over 7 orders of magnitude in the total mass, from dwarf galaxies to galaxy clusters). In papers I [28] and II [29] we systematically tested semi-analytic models used in the literature to make predictions for the DM density cores. We came to the conclusion that to obtained reliable bounds it is important to use directly the data produced by realistic SIDM simulations. The current state of the art in simulations allows doing so. To demonstrate this we have collected ensembles of haloes of all observed sizes simulated with the velocity-independent SIDM with the cross-section $1 \mathrm{~cm}^{2} / \mathrm{g}$. These simulations were performed including baryonic components and many effects of baryonic matter that can affect the properties of DM haloes and observable quantities. Analyzing these simulations we were able to make robust predictions that can be directly compared with observations.

On the observational side, we used the star's velocity dispersion data for the dwarf spheroidal galaxies, the rotation velocity data for the spiral galaxies and the weak leaning data for the galaxy clusters. We have demonstrated that it is possible to compare these data with SIDM simulations without fitting to parametric models of the DM density distribution, but rather using the estimates of the total mass that may be derived from the observational data more directly. We believe that the analysis presented here is a successful proof of concept for obtaining robust constraints on SIDM from the inner properties of DM haloes.

Our results demonstrate that it is difficult to exclude the velocity-independent SIDM cross-section with the current data. These results are mainly based on the data from dwarf galaxies and galaxy clusters, as it appears that at the relevant distances from the center these objects are more or less DM dominated. For spiral galaxies the situation is different. Although we see clear signatures of the cores (the total mass surface density demonstrates a maximum at $2-5 \mathrm{kpc}$ from the center), there is a very significant contribution of baryonic matter inside these regions. In simulations baryons make a significant effect on the DM density distribution, undoing to a large extent the effect of Dark Matter self-interaction. This means that realistic modeling of baryonic effects is crucial if we want to use the data from spiral galaxies to constrain SIDM (or maybe other DM models). In our work, we used the Eagle project [227] to describe baryonic effects. It would be extremely important to use an alternative framework to account for baryonic effects. This may potentially allow to improve the bounds on Dark Matter properties.

The robust constraints obtained here provide important input for the particle physics models of SIDM. In Section 5 we will discuss how this constraint can be combined (for a particular model) with the constraints from particle physics experiments and used to make a prediction for further searches. 


\section{Chapter 4}

\section{Indirect detection of Dark Matter with Imaging Atmospheric Cherenkov telescopes}

In the previous section, we discussed an intrinsic property of Dark Matter - the interaction of DM particles with each other. To discover DM it is useful to exploit their interaction with Standard Model particles. In this section, we will concentrate on the possible signal from annihilation of DM particles to SM matter. Below, starting from section 4.3, we will largely follow paper IV [30].

As the nature of DM is still unknown, the searches for decaying or annihilating DM can be performed in many different ways, assuming different classes of DM models. We will discuss the searches for relatively heavy DM with a mass in the $20 \mathrm{GeV}-300 \mathrm{TeV}$ range. A powerful technique for such searches is provided by IACTs [228, 229]. Interest in this technique is additionally motivated by the expectation that the current generation of IACTs will be soon superseded by a new powerful international project - the CTA [151].

Below, we introduce the IACTs technique, describe the CTA project and provide detailed estimates of the expected sensitivity of CTA for the searches for DM annihilation signal from the Galactic Center.

\subsection{Imaging Atmospheric Cherenkov telescopes}

Propagating in the atmosphere, Very High Energy (VHE) photons produce cascades (showers) of relativistic particles. Charged particles that propagate faster than the speed of light in the medium emit Cherenkov light that can be observed by ground-based telescopes. Reconstructing the properties of such a shower by its Cherenkov image allows to distinguish photonic showers from the ones produced by cosmic rays and to measure arrival direction and the energy of primary VHE photons (see e.g. [230] and references therein) as well as distinguish the showers produced by photons from those produces by protons. This is the basic idea of the IACTs technique. 


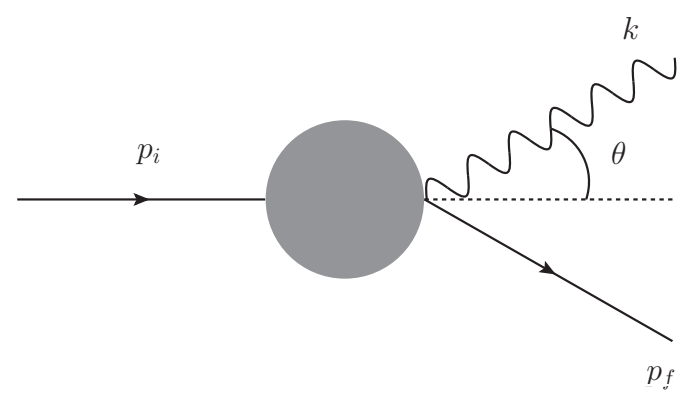

Figure 4.1: Effective Feynman diagram for Cherenkov radiation. The dark circle indicates that in fact this is a collective effect of the medium.

This technique allows to detect $\gamma$-ray photons in the energy range roughly from a few $\mathrm{GeV}$ to hundreds of $\mathrm{TeV}$ (see discussion below). At the moment, there are 4 operating IACTs:

- H.E.S.S. (High Energy Stereoscopic System);

- MAGIC (Major Atmospheric Gamma Imaging Cherenkov Telescopes);

- FACT (First G-APD Cherenkov Telescope);

- VERITAS (Very Energetic Radiation Imaging Telescope Array System).

Below we briefly remind what is Cherenkov radiation and review the physics of atmospheric showers relevant for the IACTs technique.

\subsubsection{Cherenkov radiation}

Cherenkov emission was first observed by Pavel Cherenkov in 1934 under the supervision of Sergey Vavilov [231], and later in 1937 Ilya Frank and Igor Tamm developed the theory of the Cherenkov effect [232]. The Feynman diagram of this process is shown in Fig. 4.1.

Cherenkov emission is very useful for monitoring the propagation of particles in a medium (and, in particular, observation of atmospheric showers) because as long as the conditions for Cherenkov emission are satisfied, a particle emits Cherenkov radiation continuously, it does not require scattering or an additional external field. In this way, the whole trajectory of a particle is "highlighted".

Let us remind how this effect works. A single charged particle of mass $m$ in a vacuum cannot emit a photon as energy and momentum conservation cannot be satisfied at the same time for such a process: $m \neq E_{f}+E_{\gamma}$ (rest frame), where $E_{f}$ is the energy of the particle after photon emission.

Consider the propagation of a charged particle in a medium with the speed $v$ larger than the speed of light $u$ in the medium, $v>u$. The energy and momentum conservation 
laws in the medium rest frame are:

$$
\begin{aligned}
& E_{i}=E_{\gamma}+E_{f}, \\
& \boldsymbol{p}_{i}=\boldsymbol{k}+\boldsymbol{p}_{f} .
\end{aligned}
$$

The energy and momentum of the photon in the medium are connected by the dispersion relation

$$
E_{\gamma}=u|\boldsymbol{k}| .
$$

Therefore, one can rewrite the conservation laws as

$$
\begin{aligned}
& E_{f}=E_{i}-u|\boldsymbol{k}|, \\
& \boldsymbol{p}_{f}=\boldsymbol{p}_{i}-\boldsymbol{k} .
\end{aligned}
$$

Hence,

$$
\begin{aligned}
m^{2} & =E_{f}^{2}-\boldsymbol{p}_{f}^{2}=E_{i}^{2}-2 E_{i} u|\boldsymbol{k}|+u^{2}|\boldsymbol{k}|^{2}-\boldsymbol{p}_{i}^{2}+2 \boldsymbol{p}_{i} \cdot \boldsymbol{k}-\boldsymbol{k}^{2}= \\
& =m^{2}-2 E_{i} u k+u^{2} k^{2}+2 p_{i} k \cos \theta-k^{2},
\end{aligned}
$$

where $|\boldsymbol{k}|=k,\left|\boldsymbol{p}_{i}\right|=p_{i}, \boldsymbol{p}_{i} \cdot \boldsymbol{k}=p_{i} k \cos \theta$, and $\theta$ is the angle between the momenta of the charged particle and the photon. The last equation can be written as

$$
k\left[k\left(1-u^{2}\right)+2 E_{i} u-2 p_{i} \cos \theta\right]=0 .
$$

This equation always has a trivial solution $k=0$, which corresponds to the case when the photon was not emitted. The non-trivial solution is

$$
k=\frac{2\left(p_{i} \cos \theta-E_{i} u\right)}{1-u^{2}}=\left|p_{i}=v E_{i}\right|=\frac{2 E_{i}(v \cos \theta-u)}{1-u^{2}} .
$$

$k$ is the module of momentum, therefore it should be positive.

The condition for the Cherenkov light emission is

$$
v \cos \theta>u=\frac{c}{n},
$$

where $v$ is the speed of the charged particle, $n$ and $u$ are refractive index and the speed of light in the medium.

According to Eq. (4.9), a vertically falling ultra-relativistic particle emits light into the cone with an opening angle $2 \theta \approx 2 \arccos (1 / n)$ and illuminates a circle on the ground. The refractive index for air is $n_{a}=1.000293$, which gives the maximal angle $\theta_{\max }=$ 

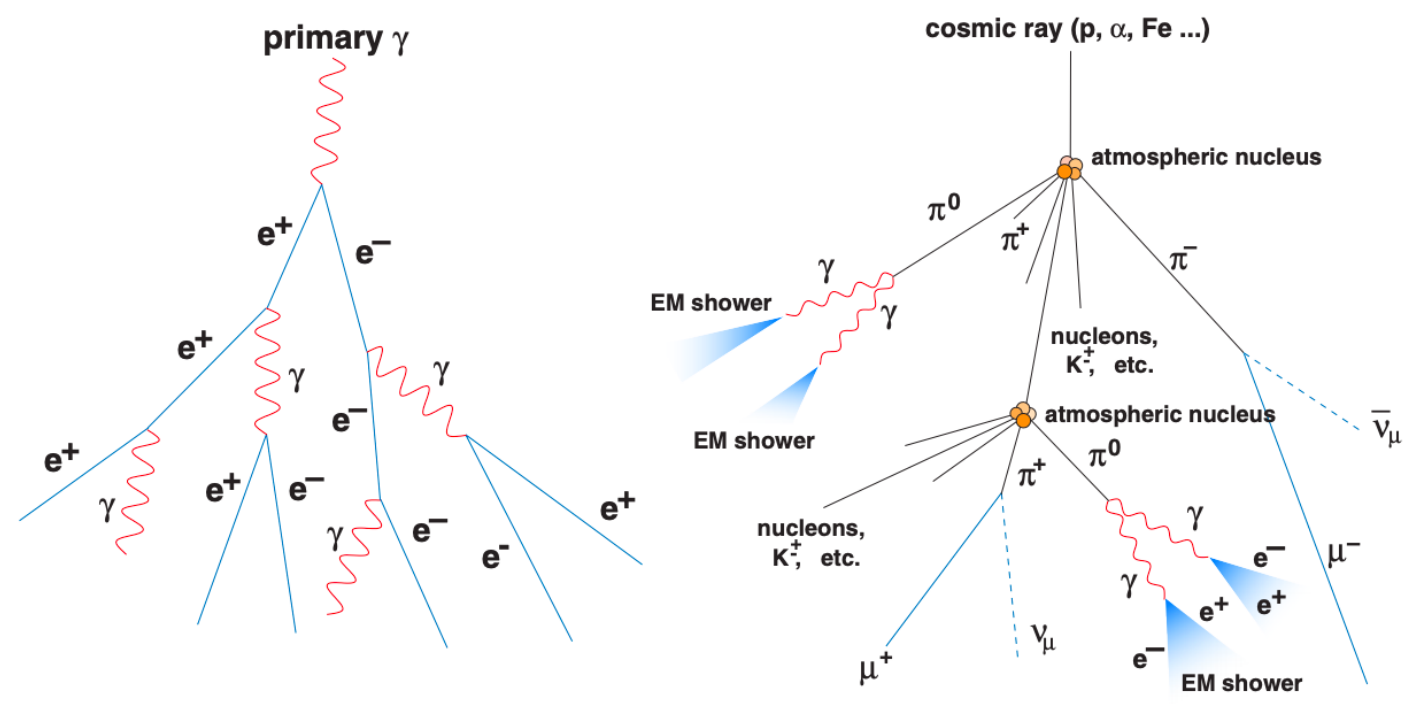

Figure 4.2: An illustration of electromagnetic (left) and hadronic showers (right) [235].

$\arccos \left(1 / n_{a}\right) \approx 1.4^{\circ}$.

The number of created photons, $N$, is given by the Frank-Tamm formula [232]

$$
\frac{d^{2} N}{d x d \lambda}=\frac{2 \pi \alpha_{\mathrm{EM}}}{\lambda^{2}}\left(1-\frac{c^{2}}{v^{2} n^{2}(\lambda)}\right)
$$

where $d^{2} N / d x d \lambda$ is the number of photons per traveled path, $d x$, of a charged particle and per wavelength interval, $d \lambda$, of photons. Numerically,

$$
\Delta N \approx 370\left(1-\frac{c^{2}}{v^{2} n^{2}\left(E_{\gamma}\right)}\right)\left(\frac{\Delta x}{1 \mathrm{~cm}}\right)\left(\frac{\Delta E_{\gamma}}{1 \mathrm{eV}}\right)
$$

From Eq. (4.10) the number of photons is bigger for smaller wavelengths, the maximum of the emission is in the ultra-violet part of the spectrum.

The Cherenkov light from air showers was predicted by Blackett [233] in 1948 and later measured by Jelly and Porter in 1963 [234]. The emitted spectrum of Cherenkov light is changed by the interaction with the atmosphere while propagating from the shower to a detector. It can scatter on air molecules, dust, water, and various aerosols in the atmosphere, and be absorbed by ozone, water and $\mathrm{CO}_{2}$. Therefore, the observed spectrum depends on atmospheric conditions. Hence, an important part of the IACT technique is to monitor atmospheric conditions. 


\subsubsection{Air Showers}

If we want to use atmospheric showers for astronomy, we have to distinguish between showers created by photons and showers created by charged particles (CRs). Indeed, unlike photons, a charged particle is deviated by cosmic magnetic fields on their way from the source to an observer. Therefore, such particles arrive to an observer from some random direction that can be arbitrarily different from the direction to the initial source where this particle was created. Hence, charged particles lose any information about their sources. That is why it is crucial for the Cherenkov astronomy to remove from the analysis showers created by charged particles (at least protons, see discussion below).

The majority of charged CRs are strongly interacting particles - protons and nuclei. Their interaction with nuclei in the atmosphere results in a so-called "hadronic showers". Electrons and photons interact with the atmosphere mainly electromagnetically, thus they create "electromagnetic showers". It appears that electromagnetic and hadronic showers have different geometrical properties. This makes it possible to distinguish between them [236]. Below, we discuss these properties of both types of showers in more detail.

\section{Electromagnetic showers}

In the left panel of Fig. 4.2 one can see an example of an electromagnetic shower. When a high-energy photon enters the atmosphere, it can scatter on an electron or on a nucleus. If the center of mass energy $E_{c m}>2 m_{e}$, an $e^{+} e^{-}$pair can be created. Then, both $e^{-}$and $e^{+}$ can emit photons via Bremsstrahlung. We have a process $\gamma \rightarrow e^{+} e^{-} \rightarrow e^{+} \gamma+e^{-} \gamma \rightarrow \ldots$ Roughly speaking, the number of particles is doubled each step of the cascade, and the energy of the particles halved. When the energy of $e^{ \pm}$drops so much that Bremsstrahlung of new photons is no more possible, the cascading stops. The "top" of the shower - its first interaction point - is typically located at an altitude $20-30 \mathrm{~km}$.

Electromagnetic showers are symmetric with respect to rotations around their axis. This is because the interactions that happen inside the shower are always the same - they are electromagnetic and create two particles out of one. We will see below that for hadronic showers the situation is different.

\section{Hadronic showers}

In showers created by primary cosmic protons or nuclei, the main role is played by the strong interaction. Unlike an electromagnetic shower, where the number of particles is doubled at each interaction, in the collisions between hadrons and atmospheric nuclei large numbers of particles can be created. These particles fly then in much wider cones as compared to the two particles created in each collision via the electromagnetic interaction. Therefore, hadronic showers are wider than the electromagnetic one (for the same energy of the primary particle). Particles created in such collisions are mesons. Most of the 


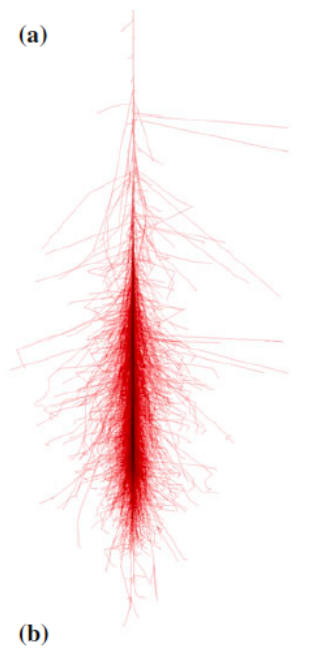

(c)

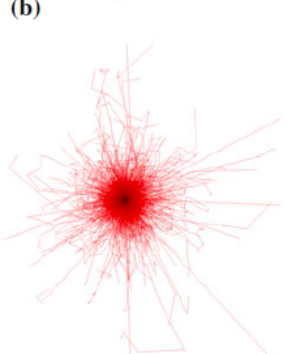

(d)

Figure 4.3: $100 \mathrm{GeV}$ showers from $\gamma$-ray (left) and proton (right). Upper panels show particles traces while lower panels correspond to the same showers seen from below. Taken from CORSIKA shower images.

mesons are pions $\left(\pi^{0}, \pi^{ \pm}\right)$, much smaller amount of kaons $(K)$ and even fewer protons, neutrons and their antiparticles, see the right panel of Fig. 4.2. The fate of these secondary particles can be very different. For example, neutral pions quickly decay into two photons $\pi^{0} \longrightarrow \gamma \gamma$. Charged pions, $\pi^{ \pm}$, have a longer lifetime and they can either collide with another atmospheric nucleus and create a secondary hadronic shower or just decay into leptons $\pi^{ \pm} \longrightarrow \mu^{ \pm} \bar{\nu}_{\mu}$.

Therefore, the width of the shower and the density of the particles in it can be very different in various directions, depending on which meson propagated in a given direction and whether it decayed or collided with a nucleus. It means that the hadronic shower will not only be wider than the electromagnetic one, but also it will be much more inhomogeneous and much less symmetric around its axis (see Fig. 4.3).

These properties allow distinguishing between the two types of showers. Simulations show that this method gives rise to misidentification between primary photons and primary CRs only in $\sim 1-5 \%$ of the cases, depending on an instrument [237]. Of course, this is an approximate number and the exact result depends on the instrument characteristics and analysis techniques. 

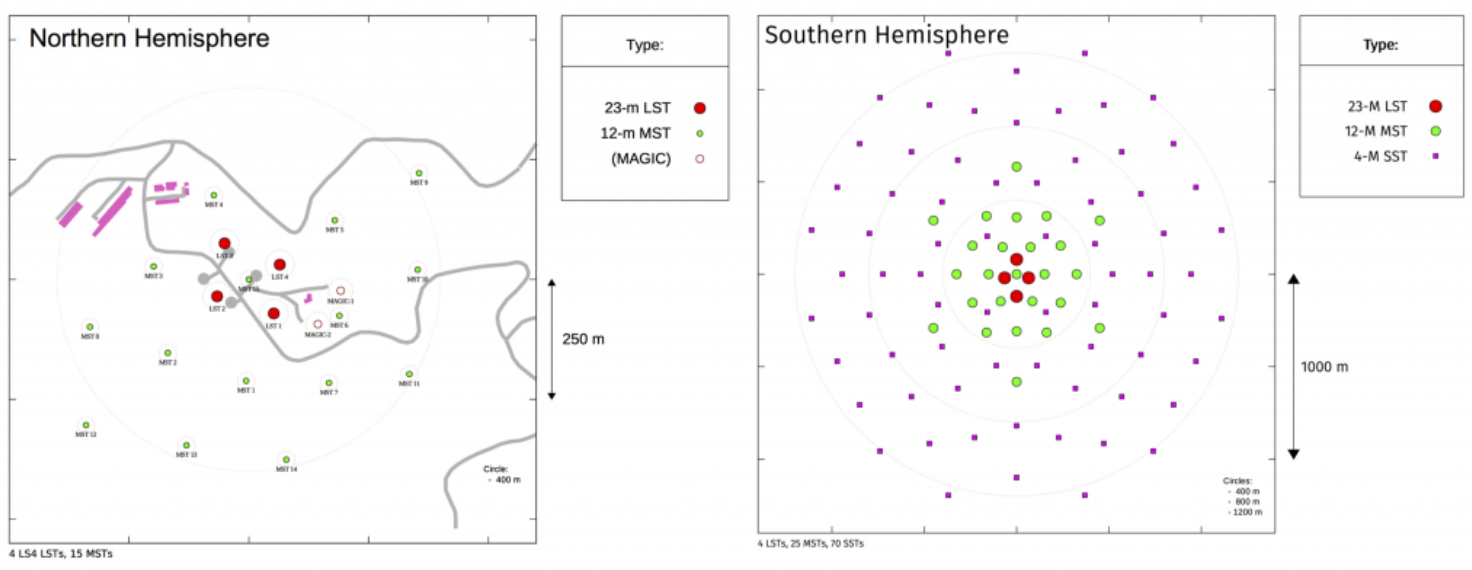

Figure 4.4: The planned location of LSTs, MSTs, SSTs and two telescopes from MAGIC for the Southern and Northern Hemispheres. Taken from [151].

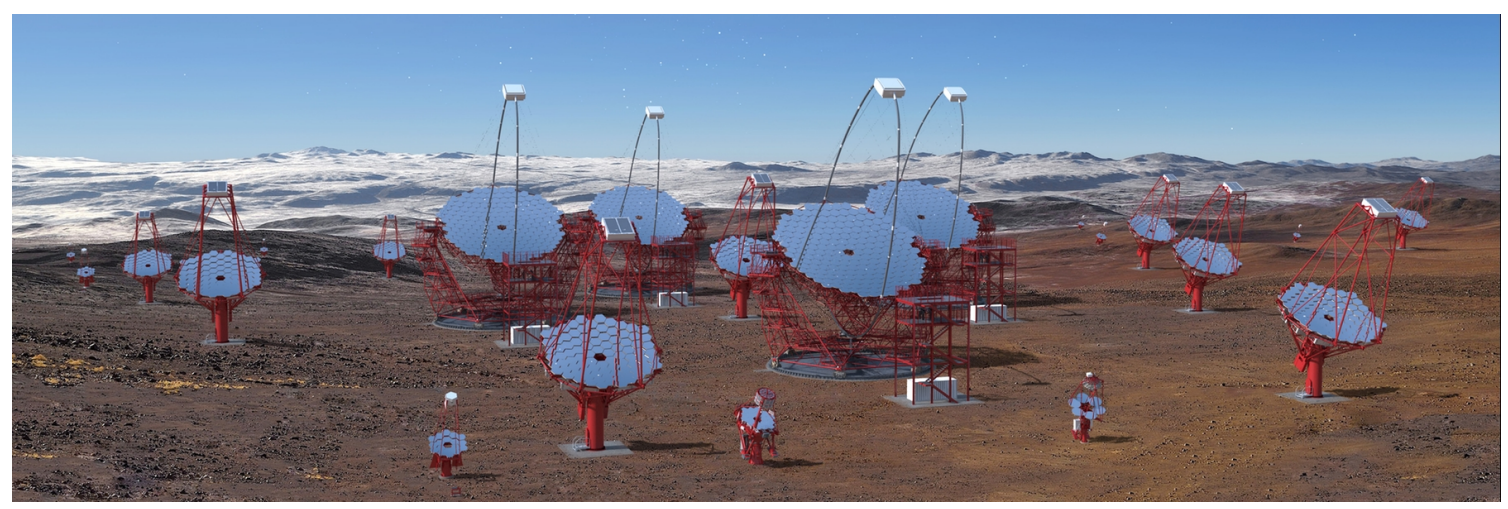

Figure 4.5: An illustration of the CTA Southern Hemisphere array. Taken from [151].

\subsection{CTA}

CTA is planned to be the next generation ground-based observatory for VHE $\gamma$-ray astronomy [151]. CTA will have two arrays: one in the Southern and one in the North Hemisphere. This is the first ground-based telescope of such a kind that opens the opportunity to observe the whole sky. The Northern Hemisphere array will be built at La Palma (Canary Islands, Spain) and will use 2 currently operating telescopes from MAGIC (see left panel of Fig. 4.4). The Southern Hemisphere array will be located in Cerro Paranal (Atacama, Chile) and will have 99 telescopes, see Fig. 4.4 (right panel).

CTA will consist of three types of telescopes with different sizes: LSTs, MSTs, and SSTs. An artistic example of the Southern Hemisphere array is illustrated in Fig. 4.5. Low energy photons produce a narrow shower with a small amount of Cherenkov photons. Such events are not rare and for this one needs only a few large telescopes (LST). The LST mirror has a diameter of $23 \mathrm{~m}$ and the energy range where LST will have the best 

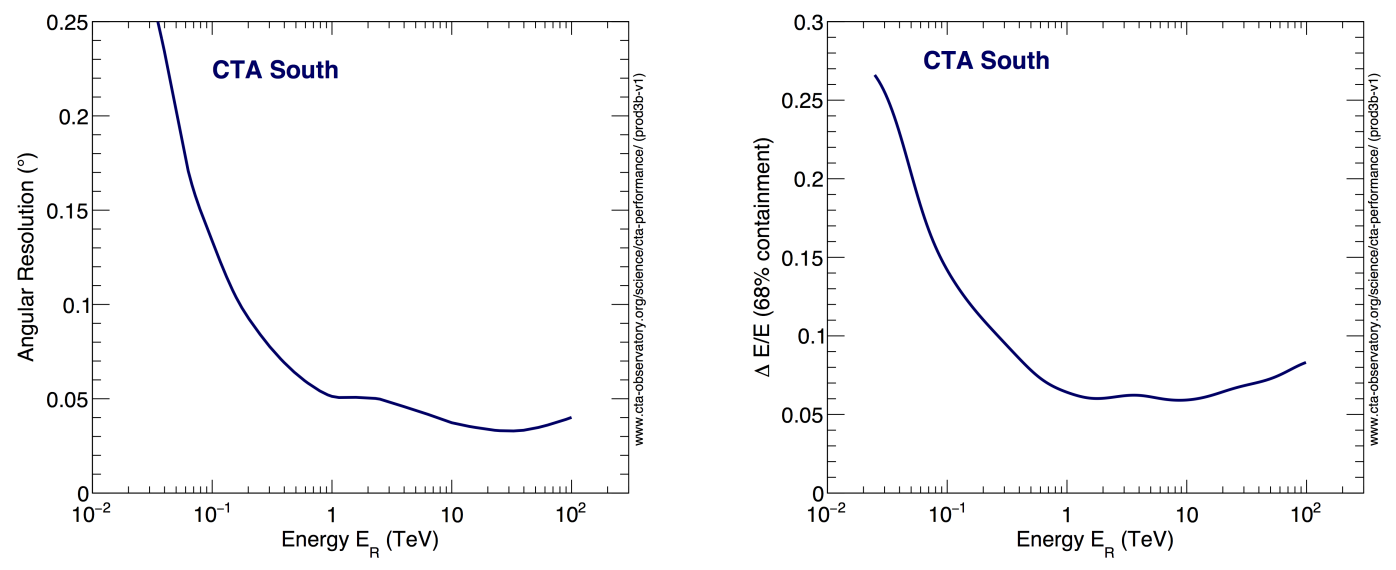

Figure 4.6: Expected angular resolution (left) and energy resolution (right) of CTA for the Southern Hemisphere. Taken from [151].

sensitivity is 20 - $150 \mathrm{GeV}$. Only 4 LSTs will be operated in both Southern and Northern Hemispheres. The MST mirror will be $11.5 \mathrm{~m}$ in diameter. 25 of MSTs are planned to built in the Southern Hemisphere and 15 in the Northern Hemisphere. The expected energy range of these telescopes is $150 \mathrm{GeV}-5 \mathrm{TeV}$. The smallest SSTs will detect the most energetic photons. As VHE events are not frequent and showers are wide, one needs to spread telescopes over a large area (several square kilometers) in order to "capture" a signal from such photons. The SST mirror has a diameter $\sim 4 \mathrm{~m}$. The center of our Galaxy is observed from the Southern Hemisphere and all 70 SSTs will be placed in Chile. The expected energy range with full sensitivity is $5-300 \mathrm{TeV}$.

CTA aims to increase the sensitivity by an order of magnitude compared to current IACTs. It will also extend the energy range from a few tens of $\mathrm{GeV}$ to about $300 \mathrm{TeV}$ and improve angular and energy resolution, see Fig. 4.6. CTA will have larger effective area and improved background rejection power [151]. As a result, the sensitivity to pointsources will be improved by at least an order of magnitude with respect to current IACTs, see Fig. 4.7. Currently, more than 170 VHE sources were discovered with IACTs and CTA is expected to detect more than one thousand VHE sources [151].

Superb characteristics of CTA as compared to the previous IACTs will also significantly increase its capability to probe fundamental physics, see e.g. [151] for a review. This includes: indirect detection of Dark Matter ([238-244], the main subject of this section), constraints on energy dependence of the speed of light (that may be induced by quantum gravity effects and other Lorentz violating phenomena [245-247]), probing the extragalactic background light [248, 249] and intergalactic magnetic field [250, 251], constraining the coupling constant of axions [252-254]. 


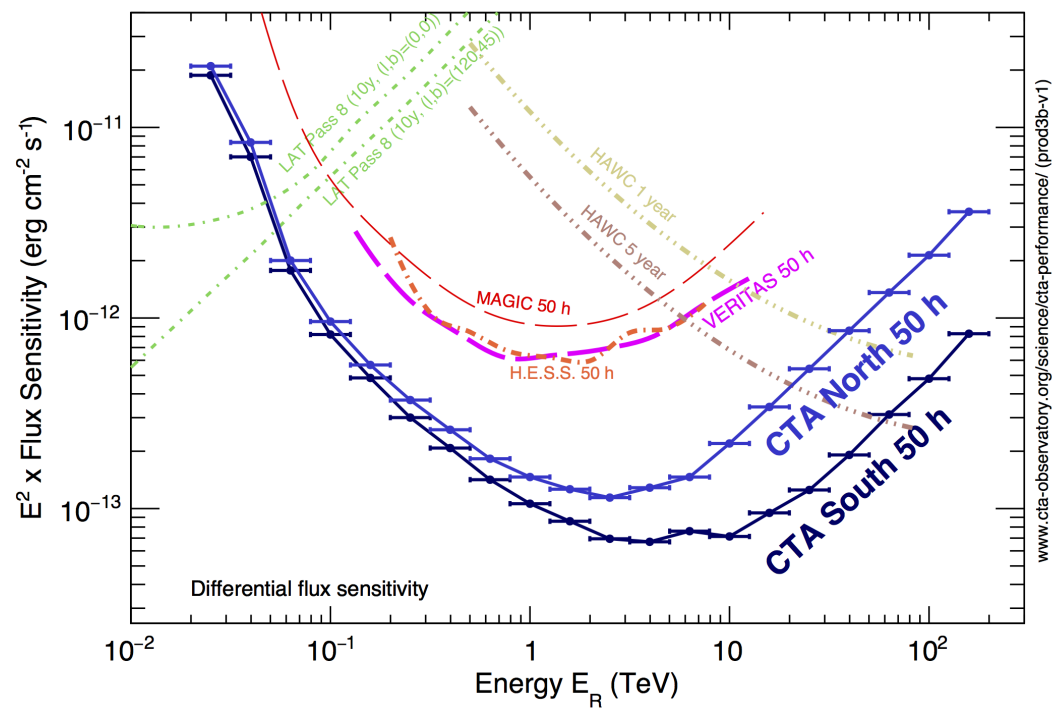

Figure 4.7: The differential flux sensitivity curve of CTA in comparison with other IACTs. Taken from [151].

\subsection{CTA simulations and data analysis with ctools}

ctools is a free software package (distributed under the GNU GPL license version 3) adopted by the collaboration as one of the analysis tools for the CTA data [255]. ${ }^{1}$ ctools has a command-line interface and the structure similar to ftools - the software for the analysis of Fermi data.

ctools is based on GammaLib, the standard tool used in $\gamma$-ray astronomy and supporting the analysis of the data from Fermi/LAT [256] and COMPTEL [257]. This allows ctools to perform the combined analysis of the data from IACTs together with the data from Fermi/LAT and/or COMPTEL.

For CTA data simulations and analysis ctools was used and tested so far mainly for point and transient sources, much less for diffuse sources like DM annihilation signal we are interested here. One of the goals of this work described in this section was to perform such testing and make sure that the package is ready for future analysis of future data for large exposure and low level of systematic errors that are required for DM indirect detection. This is the case for ctools starting from version 1.6.0.dev1. The issues we found in the previous versions were submitted and successfully fixed by the ctools team. We thank Jürgen Knödlseder for collaboration.

For our analysis we have used the following tools:

- To prepare a simulated set of mock observational data we use ctobssim that simulates

\footnotetext{
${ }^{1}$ ctools can also be used for the scientific analysis for H.E.S.S., MAGIC or VERITAS if the response functions and the data itself are provided in the correct format.
} 
event lists for observations. As input, this tool uses a model of observation and the response functions of the instrument. Which response functions to use depends on the observation time, CTA North or CTA South arrays. There are average response functions for full telescope array and specific ones for small, large or medium telescopes. For example, "South_z20_average_50h" means the average response function for all telescopes for 50 hours and observation of a source at 20-degree zenith angle. Input model contains specified astrophysical sources and instrumental background. For a given model the events (both from astrophysical source and from the background) are generated by random number generator from GammaLib. One can generate different realizations of the event list for the same observation and the same model, specifying the seed value for different realizations by the seed parameter. As the output the tool produces a FITS file that contains the event list and Good Time Intervals (i.e. the time intervals during the observation when the data are not affected by e.g. atmospheric conditions and can be trusted).

- To find the allowed values of the free parameters of a given physical model we use the ctlike tool. This tool performs the procedure of the maximum likelihood fitting of the IACTs data. It can use both real data and mock data produced by ctobssim. For our analysis we use mock data of the background prepared by ctobssim as an experimental data. ctlike tool can use both the whole data altogether and the data binned by spatial and/or energy bins. It can also use data from different observations and different instruments. If we stack different observations together ctlike will use the average response function, while if we keep the observations separately the response functions of the corresponding instruments should be used. ctlike will then use the joint likelihood function for all observations and instruments to constrain the physical parameters of the model:

$$
-\ln \mathcal{L}(M)=-\Sigma_{i} \ln \mathcal{L}_{i}(M) .
$$

Likelihood is defined in ctlike assuming Poisson statistics. However, there is an option to use Gaussian statistics in some cases.

During the fit ctlike will vary all the parameters that have flag free="1". Other parameters will be frozen. To specify the model parameters for which Test Statistics (TS) will be calculated one can use the attribute tscalc=" 1 ":

$$
\mathrm{TS}=2 \ln \mathcal{L}(M)-2 \ln \mathcal{L}\left(M_{-j}\right),
$$

where the best fit likelihood of the model $M$ is $\mathcal{L}(M)$ and the maximum likelihood for the same model without the component $j$ is $\mathcal{L}\left(M_{-j}\right)$.

The output of ctlike is an XML file describing the model with best-fit values of all 
its components, with the statistical uncertainty for all parameters that were adjusted during the fit and the TS value when it was requested. This XML file can be used as an input file for other tools that require a model definition as input, e.g. ctobssim or ctmodel.

- ctmodel creates a 3-d count map for a model (this 3-d map is also called a model cube). This map consists of the number of counts for each point on the sky (parameterized by a pair of coordinates, i.e. either (Galactic longitude, Galactic latitude) or (Right Ascension, Declination)) for each energy. The energy dimension makes the map 3-d. The output of ctmodel is a FITS file containing the number of counts, the energy boundaries and Good Time Intervals of the involved observations.

\subsection{Systematics}

CTA is expected to have different kinds of systematic errors: spectral systematic or energy resolution (an uncertainty in measuring the energy of each photon), point spread function (PSF), imperfect knowledge of the effective area at each energy, etc.

Here we will study the systematics that results in the imperfect measurement of the number of events. This can occur due to e.g. misidentification of events, for example, we can mix up a proton-induced and photon-induced showers. We will assume that these systematics are at the level of $1 \%$ of the total number of events. In fact, when CTA starts to collect data, the systematic error can be as large as $10 \%$, but the goal of CTA is to reach $1 \%$ level and for this reason, we use this number as a benchmark.

Below we describe a simple approach which allows handling the systematic uncertainties without modifications of the source code of ctools. We demonstrate later (see Section 4.5) that this approach gives the results consistent with the likelihood-modification approach, described also in [238]. In the simple approach described here, we can not take into account possible correlations between energy and spatial bins (unlike in the likelihood-modification approach). These correlations, however, are difficult to estimate before the instrument is built and, as two approaches give consistent results, we believe that our method still allows estimating the effect of systematic on the sensitivity.

Our approach is based on the simple observation that statistical errors grow with the number of observed events as

$$
\delta N_{\text {stat }}=\sqrt{N_{\text {signal }}+N_{\text {bg }}} \sim \sqrt{T_{\text {obs }}}
$$

where $N_{\text {signal }}$ and $N_{\text {bg }}$ are the number of signal and background events, and $T_{\text {obs }}$ is the observational time. Systematic uncertainty is usually a fraction of the total number of 
observed events and grows linearly with $T_{\text {obs }}$, i.e.

$$
\delta N_{\text {sys }}=\alpha \cdot\left(N_{\text {signal }}+N_{\text {bg }}\right) \sim T_{\text {obs }},
$$

where $\alpha$ defines the level of systematics. Consequently, with the increasing of observational time, the signal to noise ratio (SNR) approaches a constant

$$
\mathrm{SNR}=\frac{N_{\text {signal }}}{\delta N_{\text {stat }}+\delta N_{\text {sys }}} \simeq N_{\text {signal }} / \delta N_{\text {sys }} \sim \text { const. }
$$

After a certain time, $T_{\max }$, the SNR remains roughly a constant which indicates entering the systematics-dominated regime in which it makes no sense to increase the observation time further. $T_{\max }$ can be found as the time at which the systematic and statistic errors become comparable, i.e. $\delta N_{\text {stat }}=\delta N_{\text {sys }}$. Obviously, the requirement of maximal observational time $T_{\max }$ can be translated to the corresponding requirement on a maximal number of detected events $N_{\max }=\alpha^{-2}$. We argue that in the case one can split the data into a set of statistically independent bins (spectral and/or spatial) $N_{\max }$ condition has to be applied not to the whole dataset, but to each individual bin (we assume for simplicity that if our bins are large enough, we can neglect correlation between them, see the comment about taking into account correlations above).

In the case of CTA, the spectral bins of the data can be as small as the CTA energy resolution and the spatial size of bins is determined by the PSF of the instrument. For the analysis below we assume the energy resolution to be $\delta E / E=10 \%$ and the spatial bins - squares with $0.2^{\circ}$ side, see Fig. 4.6 and Ref [151]. This resulted in 78 (spectral) $\times 25 \times$ 25 (spatial) statistically independent bins in our analysis. For each of the bins, we reduced the exposure time for data simulation in order to have not more than $N_{\max }=10^{4}$ photons in each of these bins, which corresponds to $1 \%$ of systematics expected during the CTA operation. The reduction of the number of counts in each of these bins guarantees that any analysis performed with the data is not dominated by systematic uncertainties.

Summarizing, to take into account systematic uncertainties in this simplified way with ctools one should:

- split the data over a set of energy/spatial statistically independent bins;

- if the bins are small enough statistical errors become of the size of the desired systematic errors (i.e. the number of counts does not exceed a certain number $N_{\max }$;

- when needed, reduce the effective exposure for each (separate) bin accordingly;

- proceed to calculate the limits with statistics errors, with ctools/any other software.

Of course, the current method, being very simple, has both its advantages and disadvantages. 
- The advantages include:

- The method is very easy to implement with ctools - it does not really need any modifications of the code, only a proper selection of observational times in every energy (and spatial) bin;

- The method is very intuitive and quick, it does not require computational time significantly larger than the time for the standard ctools analysis;

- It is based on very simple assumptions about PDF and covariance matrix - this is useful as these quantities are difficult to know exactly before the instrument is built.

- It is well-suited for searches for localized features. The spectral shape of DM annihilation signal is model dependent, the method would work the best for the case of DM lines. However, for any model, the signal has a maximum related to DM mass.

- Studying the residuals after changing the observation times and finding the times when the residuals become close to Gaussian allows estimating the level of systematics in the real data "experimentally".

- Implementation of the method can directly affect observational strategy and free a part of valuable time of the LST (see discussion below).

- Limitations of the method:

- It is not applicable to large-scale systematics, like an additional unknown systematic difference between low and high energies.

- Systematic uncertainty added to the likelihood function via covariance matrix has well studied statistical properties, which are less obvious for our method. It is also difficult to include correlations. To the end, we perform a comparison between the results of the two approaches and make sure that they are consistent.

We would like to stress again, that the simple approach that we suggest, described above, can be useful for every-day operations of CTA. Namely, a sufficiently large enough $N_{\text {max }}$ can be reached with LSTs at $150 \mathrm{GeV}$ energies already after a relatively short observational time while at higher energies the same $N_{\max }$ will be reached for significantly longer exposures with MSTs/SSTs. Instead of wasting further LST time, this opens the possibility to split the observation time and partially use it to observe other types of sources at low energies. As an example, for $1 \%$ of systematics, we calculate with ctools the number of photons in each energy bin and the maximal observational time $T_{\max }$ shown in Fig. 4.8. 


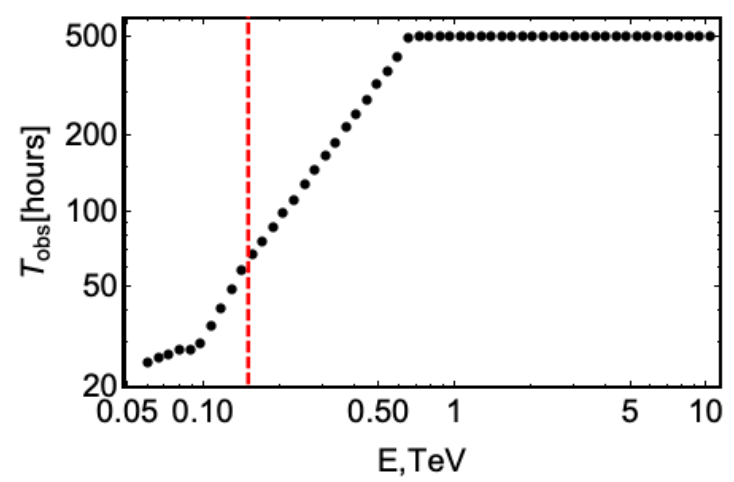

Figure 4.8: Observation time limits with the requirement that in each energy bin the number of events is equal or less than $10^{4}$ events. Starting from $1 \mathrm{TeV}$ the number of events in each bin is smaller than $10^{4}$ even for $500 \mathrm{~h}$, therefore the observation time should not be reduced and is kept to be $500 \mathrm{~h}$. The red line indicates $150 \mathrm{GeV}$ - the energy where LSTs stop to operate. Taken from paper IV [30].

In the forthcoming CTA collaboration paper we are going to systematically develop also an alternative method of constraining DM annihilation by the Galactic Center observations with CTA (see paper IV [30]). This method is not using ctlike for the statistical analysis but rather adopts the Swordfish package [258]. This approach allows to describe systematics at the level of likelihood and take into account the correlations between spatial and energy bins. In the paper, we will describe this approach in details and will perform a systematic comparison between two approaches. Below we will just quote preliminary results of this work and compare them with the results of the approach described here.

\subsection{Sensitivity for DM annihilation from the Galactic Cen- ter and comparison with other methods}

Our goal is to get the sensitivity of DM annihilation in the Galactic Center for WIMP particles and get the limit on the cross-section of the annihilating DM. As discussed above, we need to detect a possible signal against the backgrounds (CRs and GDE). We will use ctools for our analysis.

For this we need to translate the limit on the flux into the limit on the DM-annihilation cross-section [38]. We remind (see Eq. (2.13) in Section 2.5) that the predicted flux from DM annihilation in astrophysical object is

$$
\frac{d F_{\gamma}}{d \Omega d E_{\gamma}}=\frac{1}{8 \pi M_{\mathrm{DM}}^{2}} \sum_{f}\langle\sigma v\rangle_{f} \frac{d N^{f}}{d E_{\gamma}} \frac{d J(\theta)}{d \Omega},
$$

where $F_{\gamma}$ is the predicted flux, $J$ is the $J$-factor, see Eq. (2.14) (a function of DM density 
distribution in the object), $\theta$ is the angle between the direction to the center of a given astrophysical object and the line of sight (1.o.s.), $\frac{d N^{f}}{d E_{\gamma}}$ and $\langle\sigma v\rangle_{f}$ are the energy spectrum of photons per annihilation and the annihilation cross-section into a primary channel $f$, respectively.

To calculate the predicted signal from the Galactic Center we need detailed knowledge about DM around the Milky Way center. This is, unfortunately, the source of serious uncertainties.

\subsubsection{Uncertainties in the expected signal from the Galactic Center}

As we discussed in Section 2.5.2, the $J$-factor is proportional to the density of DM squared and, therefore, is very sensitive to the details of DM distribution, in our case in the Galactic Center. There are different ways to constrain this distribution observationally (see e.g. [259-261]). These efforts are complicated by the presence of the stellar Bulge and Bar that affect the motion of the stars and gas in the inner part of the Galactic disk and make it difficult to use rotational velocities to reconstruct the enclosed mass. To reduce this uncertainty various groups use additional data from Gaia [262] and several surveys studying the kinematics of stars in the Galactic Center (see details below). The velocities of stars can be used to constrain the total mass profile and microlensing data to determine how much of the mass is in stars.

In particular, Portail et al. 2017 [260] and Wegg et al. 2018 [259] use different tracers to constrain the total mass in the Bulge region: Portail et al. 2017 [260] uses BRAVA [263, 264] and OGLE [265] surveys for the Bulge, and ARGOS [266, 267] survey for the Bar region, while Wegg et al. 2018 [259] uses Gaia II data for the RR Lyrae stars. They obtain consistent results for the total mass in the inner $2.5 \mathrm{kpc}$ around the Galactic Center. Also, both papers use the same microlensing data from VVV, UKIDSS and 2MASS [268] to constrain the contribution of stars into the total mass. As one can see in Fig. 4.9, the results obtained by these two methods are in good agreement for DM. The resulting DM mass fraction in the Bulge region is equal to $17 \pm 2 \%$ according to these studies $[259,260]$.

On the other hand, this conclusion is not supported by the analysis of [261]. These authors agree with the reliability of the kinematical data for the total mass estimation but question the precision of the measurement of stellar mass based on the number of microlensing events in the current infrared surveys. With this increased uncertainty suggested by [261], the mass fraction of DM in the region of interest can be estimated only as being between $6 \%$ and $45 \%$. These authors, nevertheless, agree that the forthcoming Wide Field InfraRed Survey Telescope (WFIRST) [270] is expected to significantly reduce this uncertainty.

One could naively think that if we are looking for a signal from the annihilation of 


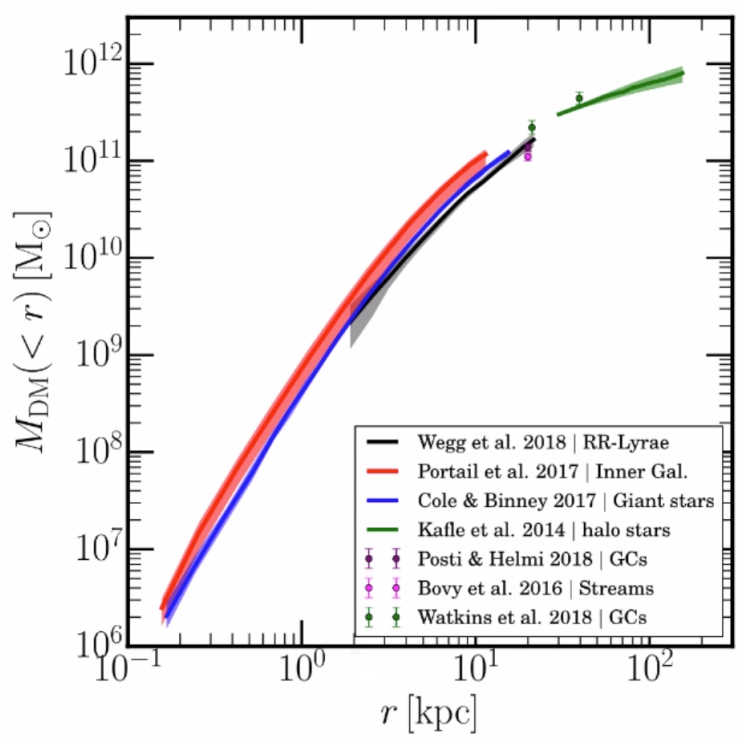

Figure 4.9: The mass profile for the Milky Way, based on different observables. Taken from J.Read, 2019 [269].

WIMPs, we have to assume that DM is cold, DM density profiles are cuspy and one uses $\Lambda \mathrm{CDM}$ simulations to predict it. In reality, this is not possible. Indeed, the scales we need to resolve are comparable with the trust radius even for high-resolution Aquarius simulations [95, 212]. Apart from the numerical precision, the DM density distribution at such small scales strongly depends on the assumptions about baryonic physics. Indeed, even for dwarf galaxies, that are largely DM dominated, baryonic effects can create significant cores (see e.g. [271]). Therefore, it is safer to constrain DM distribution from observations.

To discuss quantitatively the effect of the uncertainty in the DM mass measurement on the DM annihilation signal we will use two DM density profiles that are consistent with the DM measurement at large radii, varying the DM mass in the center within the uncertainty suggested by [261], namely:

- the Einasto profile

$$
\rho_{\text {Einasto }}(r)=\rho_{s} \exp \left(-\frac{2}{\alpha}\left[\left(\frac{r}{r_{s}}\right)^{\alpha}-1\right]\right),
$$

where we take $\alpha=0.17$ and $r_{s}=20 \mathrm{kpc}$, and $\rho_{s}=0.00214 \mathrm{M}_{\odot} / \mathrm{pc}^{3}$,

- a cored Einasto profile

$$
\rho_{\text {coredEinasto }}(r)= \begin{cases}\rho_{\text {Einasto }}\left(r_{c}\right) & \text { if } r \leq r_{c} \\ \rho_{\text {Einasto }}(r) & \text { if } r>r_{c}\end{cases}
$$

where we assume two different core radii of $r_{c}=0.5,1.0 \mathrm{kpc}$. 


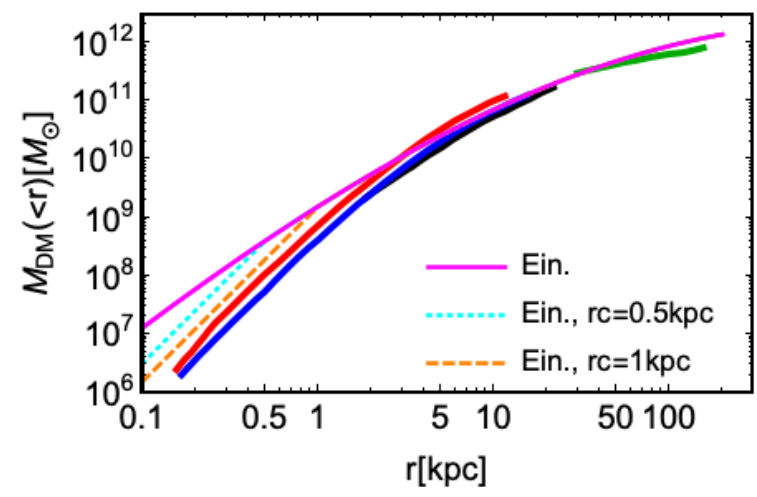

Figure 4.10: The comparison of mass profiles for the Milky Way from observation (see Fig. 4.9) and for the Einasto profiles described above.
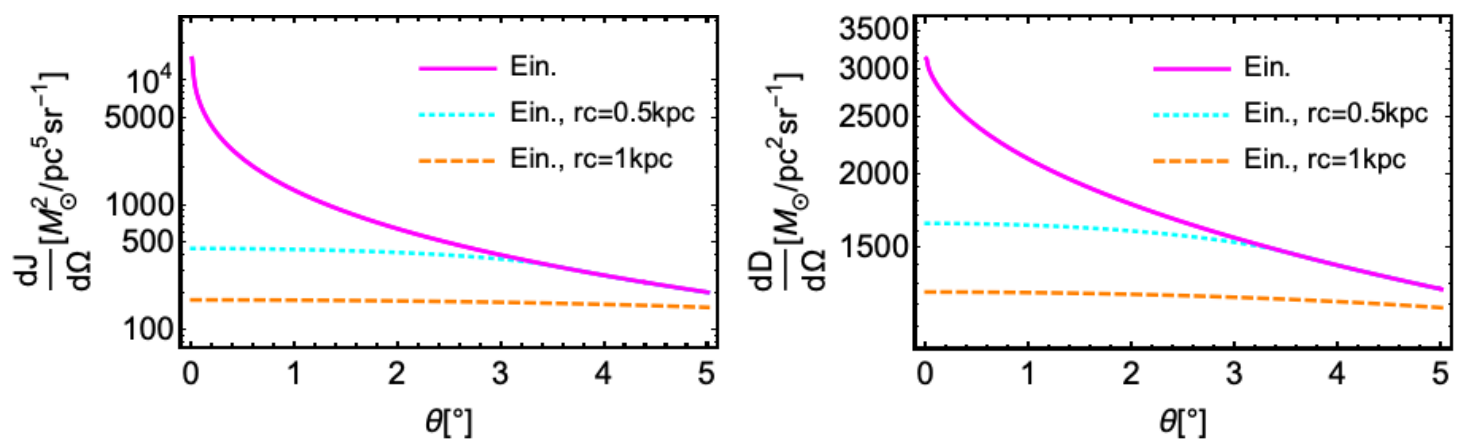

Figure 4.11: $J$-factors and $D$-factors for the Einasto profile with $\alpha=0.17, r_{s}=20 \mathrm{kpc}$ and $\rho_{s}=0.00214 \mathrm{M}_{\odot} / \mathrm{pc}^{3}$ and for cored Einasto profiles with $r_{c}=0.5 \mathrm{kpc}$ and $1 \mathrm{kpc}$.

These profiles together with the results from Fig. 4.9 are shown in Fig. 4.10.

The $J$-factors and $D$-factors for these profiles are shown in Fig. 4.11. We see that the difference between different DM profiles in the central $0.1^{\circ}$ (roughly one PSF of CTA) is a factor of 100 for $J$-factor and a factor of 3 for $D$-factor. For the cored Einasto profile with $r_{c}=1 J$-factors and $D$-factors are almost constant in the FoV of CTA.

\subsubsection{Calculation of sensitivity}

It is planned to devote about $500 \mathrm{~h}$ of the CTA observational time to study the Galactic Center region [151]. There are different options for the observational strategy. The simplest one is a single observation centered at the Galactic Center with the observation time $T_{\text {obs }}=500 \mathrm{~h}$ and the FoV radius $3^{\circ}$. Another option is a survey that consists of 9 individual pointing positions with centers $\left(l= \pm 1^{\circ}, 0^{\circ}, b= \pm 1^{\circ}, 0^{\circ}\right)$ in the Galactic coordinates, and the total observation time of the Galactic Center survey is $T_{\text {obs }}=525 \mathrm{~h}$ with the pointing radius of $3^{\circ}$. In the following we will use the simplest scenario of a single pointing for our estimates. 
For the calculation of sensitivity, we also need to assume the concrete annihilation channel. The spectra corresponding to different channels are shown in Fig. 2.14, see detailed discussion e.g. in paper IV [30]. Here, as an example, we will use the spectrum for DM annihilating to $W W$ bosons, without EW corrections (see Fig. 2.14 for the spectral shape). To generate the mock data we perform MC simulations using ctobssim. We repeat our simulations for 20 realizations with random seed values (we checked that 20 realizations are enough for convergence). Alternatively, we can produce model predictions (taking into account the telescope response function and other properties for given observations) using ctmodel. This prediction can be used as an Asimov data set [272]. We later compare the constraints obtained by averaging over these realizations with those based on the Asimov data set.

We perform simulations for the CRs background, $F_{\mathrm{bg}}$, within the $3^{\circ}$ ring. To reduce computational efforts and minimize uncertainties in the effective area we use the energy range $60 \mathrm{GeV}-100 \mathrm{TeV}^{2}$. We also repeat this adding GDE. For this we use the so-called Gamma model of GDE derived in [273]. This model fits both Fermi LAT and HESS data. For the purely statistical analysis, we assume the exposure of 500 hours in all energy bins. The binning of the data, as well as the method to take into account systematic errors, is described in Section 4.4. For this, we do the simulation choosing different observation times in different energy bins.

Then we fit this mock data by a background model + with added DM signal $F_{\text {total }}=$ $F_{\text {bg }}+F_{\text {signal }}$, where $F_{\text {signal }}$ is

$$
F_{\text {signal }}=\langle\sigma v\rangle \operatorname{Spectral}(E) \sum_{i} \operatorname{Spatial}_{i} \Omega_{i}
$$

where $\Omega_{i}=0.25^{\circ} \times 0.25^{\circ}$ is the angular size of 1 pixel and the sum over all pixels in the map $\sum_{i}$ Spatial $_{i}$ should be equal to $1^{3}$.

We performed the binned likelihood analysis fitting the model, described above, to the simulated data using ctulimit. For each $M_{\mathrm{DM}}$ we derived the $2 \sigma$ upper bounds on $\langle\sigma v\rangle$. In Fig. 4.12 we present the results obtained with and without systematic errors included. We also compare our results with the constraints obtained using Asimov data set and treating systematics statistically (including it in the definition of the total likelihood, see discussion above [272]). We demonstrate that our simple approach to systematic errors described in Section 4.4 gives results consistent with the likelihood approach.

We see that even including effects of systematics (at $1 \%$ level) and GDE the sensitivity goes below the thermal cross-section. As we saw above at smaller energies the sensitivity is dominated by systematics, while at larger energies it is still defined by statistics, even

\footnotetext{
${ }^{2}$ Below $60 \mathrm{GeV}$ the number of photons per bin is so large for a $500 \mathrm{~h}$ observation that it requires to increase numerical resolution in ctools. This makes it numerically expensive. This limitation does not affect the results in any significant way.

${ }^{3}$ As required by the ctools model-factorization approach, see e.g. http://cta.irap.omp.eu/gammalib/users/.
} 

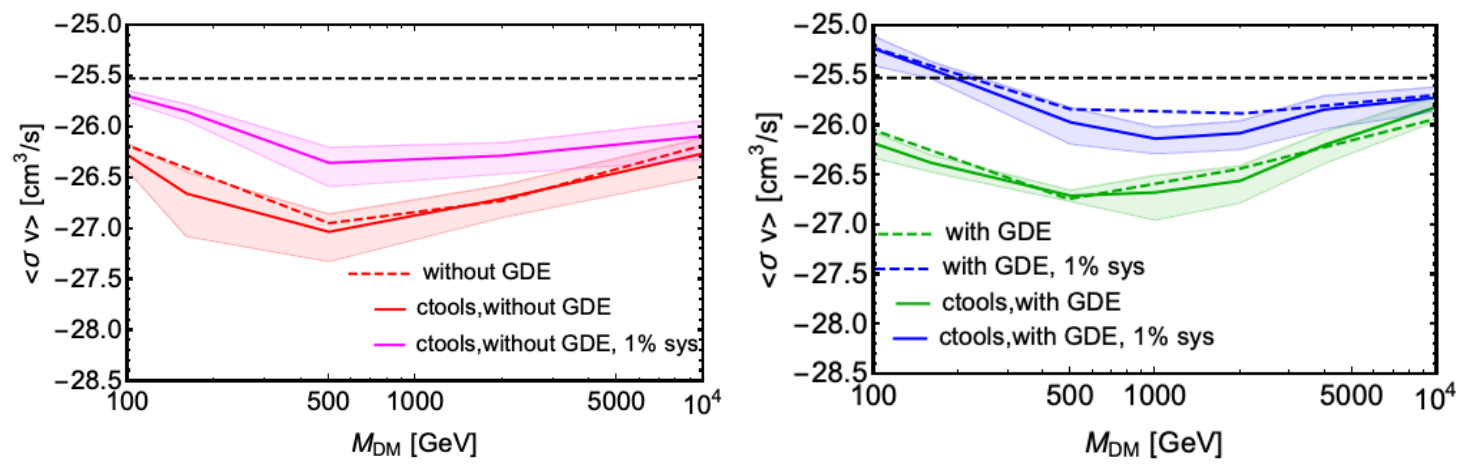

Figure 4.12: Comparison of the limits on $\langle\sigma v\rangle$, based on the likelihood approach (dashed lines) with the limits from ctools (solid lines) for DM with the Einasto profile, $W W$ channel, and $T_{\text {obs }}=500 \mathrm{~h}$ with (right) and without (left) GDE. The agreement between ctools and the likelihood method (dashed lines) is good within the uncertainties over different realizations (shaded regions) for both cases with and without systematic errors. Taken from paper IV [30].

for $500 \mathrm{~h}$ observation. Our results are obtained for a certain model of GDE (see above). Additional uncertainty coming from the modeling of GDE may be quite significant and affect the morphological analysis. The energy spectra of GDE and of DM annihilation are however quite different.

\subsection{Conclusion}

We have tested the software package ctools adopted by the CTA collaboration for the detection of a possible DM annihilation signal. We have chacked that, starting from the version 1.6.0-dev1 (where all the problems that we identified have been already fixed), ctools is ready for such an analysis. We discussed here only the signal from the prompt emission of photons. It would be potentially interesting to include in the analysis a possible contribution from the Inverse Compton effect, having different spacial morphology and spectral properties.

Although the treatment of systematic errors is not yet implemented in ctools, it is possible to take them into account adopting a simple physical approach that does not require any modifications of the code. This method, based on a limiting number of counts in each energy/spacial bin, suggests that for long exposure observations of the Galactic Center the LSTs should be used only for less than 50 hours (even if the whole observation may be much longer), see Fig. 4.8. Indeed, the number of counts in the energy bins below $150 \mathrm{GeV}$, where the LSTs operate, is huge and the data will quickly become dominated by systematics, even if it is at the very optimistic level of $1 \%$. This may potentially free a significant amount of the LSTs time for other science goals, where the most interesting signal is expected at low energies (e.g. observing distant active galaxies or binary 
systems [274, 275], see also [151] for a review).

Our results demonstrate that assuming an optimistic Einasto profile for the DM density distribution in the Milky Way and $W W$ annihilation channel, with 500 hours of the Galactic Center observations with CTA we can probe the annihilation cross-section below its thermal value. This is true if the level of systematics $1 \%$ will be achieved by the CTA collaboration. Of course, the main uncertainty to this conclusion comes from the measurement of the Dark Matter mass around the Milky Way center. If these recent claims of $[259,260]$ are confirmed, CTA will be significantly less sensitive. In such a case it may be more efficient to use dwarf spheroidal galaxies as a primary target for indirect DM detection with CTA. 


\section{Chapter 5}

\section{Light Dark Matter with a scalar mediator}

\subsection{Introduction}

The constraints on the properties of Dark Matter particles discussed in the previous sections were to some extent model independent (at least, applicable to rather wide classes of particle physics models containing Dark Matter). In this section, we would like to discuss how different bounds (astrophysical, cosmological, accelerator, and direct detection bounds) can be combined together for a particular model. Such a combination allows to narrow down the parameter space of a model and optimize the strategy of searches for Dark Matter particles for each experiment. We will choose a simple model of a WIMPlike fermion that is coupled to the Standard Model through the so-called "scalar portal", i.e. a scalar field that "mixes" with the Higgs boson. In what follows, we will mainly consider a fermion with a mass $m_{\chi} \lesssim 5 \mathrm{GeV}$, i.e. what is often called "Light Dark Matter". This type of DM candidates attracted additional interest in recent years as this part of the parameter space was much less studied experimentally. Therefore, it is important to find new ways to explore it (see for example [276]).

A scalar mediator that enables the coupling of DM to the SM sector can also mediate DM self-interaction via Yukawa coupling with the DM fermion. In this case, DM becomes self-interacting, as discussed in Section 3. We will be interested in the case when the scalar mediator is lighter than the Dark Matter fermion. In this case, the cross-section of selfinteraction may become velocity dependent. This can be interesting from an observational point of view as the effect of self-interaction can be stronger in smaller objects (e.g. dwarf galaxies) and it can be potentially observable in small galaxies even if the constraints from merging galaxy clusters will get stronger [83].

This kind of model is relevant also in the context of searches for relatively light longlived particles that attract more and more interest in recent years [277]. At accelerators these searches are currently performed and planned both at the LHC (see e.g. [278280]) and the so-called "intensity frontier experiments" (e.g. SHiP [281-283], MATHUSLA [284], etc.). For the sake of a proof of concept, we will consider only the simplest 
model.

\subsection{Model description}

Let us consider the simplest Lagrangian of a new scalar $S$ and a new Dirac fermion $\chi$ that can play a role as the DM particle (see e.g. [285] and references therein)

$$
\mathcal{L}_{S / \chi+S M}=\frac{1}{2} \partial_{\mu} S \partial^{\mu} S-\frac{m_{S}^{2} S^{2}}{2}+\bar{\chi}\left(i \not \partial-m_{\chi}\right) \chi-\mu S H^{\dagger} H-\lambda S^{2} H^{\dagger} H-g_{\chi} S \bar{\chi} \chi .
$$

Here $m_{S}$ and $m_{\chi}$ are the masses of the scalar and the DM fermion, $H$ is the Higgs doublet of the Standard Model. This model has the $U(1)$ symmetry that makes the DM fermion stable. We assume that the mass of the scalar particle is smaller than that of the new fermion, $m_{S}<m_{\chi}$, and the coupling constant $\mu \ll m_{h}$. As mentioned above, we will be mostly interested in the masses of the fermion $m_{\chi}<5 \mathrm{GeV}$. In this theory, there are two interaction terms: the first one couples the "dark" fermion to the Standard Model through the scalar mediator, while the second term defines the interaction between the fermions.

The interaction terms $\mu S H H+\lambda S^{2} H H$ form the so-called "scalar portal" - interactions between the new scalar $S$ and the SM that do not require the coupling constant with a negative mass dimension. For the phenomenology that we will discuss below, only the term $\mu S H H$ will play a role.

After spontaneous symmetry breaking, the Higgs doublet in the unitary gauge becomes

$$
H=\left(\begin{array}{c}
0 \\
\frac{v+h}{\sqrt{2}}
\end{array}\right)
$$

where $v$ is the vacuum expectation value and the scalar $h$ describes the physical Higgs boson. Plugging it into the Lagrangian (5.1) one gets

$$
\mu S H^{\dagger} H=\mu \frac{v^{2}}{2} S+\mu v S h+\frac{\mu}{2} S h^{2} .
$$

The first term in Eq. (5.3) contains only one field - the scalar field $S$ and can be eliminated by a shift of the scalar field, i.e.

$$
-\frac{m_{S}^{2} S^{2}}{2}-\mu \frac{v^{2}}{2} S=\left|S \rightarrow S-\frac{\mu}{2} \frac{v^{2}}{m_{S}^{2}}\right|=-\frac{m_{S}^{2} S^{2}}{2}+\frac{g_{S H H}^{2}}{8} \frac{v^{4}}{m_{S}^{2}} .
$$

The third term in Eq. (5.3) is the interaction between two Higgs bosons and the new scalar $S$. This term is irrelevant for the phenomenology of particle physics experiments as it contains two Higgs bosons and therefore can not contribute neither to creation of the light scalars nor to their decays of scattering in real experiments. 


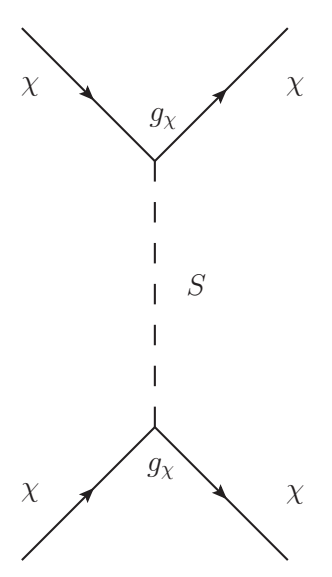

Figure 5.1: Feynman diagram of the DM self-interaction in the model (5.1).

Finally, the second term in Eq. (5.3) is the mass mixing between the Higgs boson and the new scalar $S$. Again, it can be eliminated using the rotation of the Higgs boson and $S$ fields,

$$
-\frac{m_{S}^{2} S^{2}}{2}-\mu v S h-\frac{m_{h}^{2} h^{2}}{2}=\left|\left(\begin{array}{l}
S \\
h
\end{array}\right) \rightarrow\left(\begin{array}{cc}
\cos \theta & \sin \theta \\
-\sin \theta & \cos \theta
\end{array}\right)\left(\begin{array}{l}
S \\
h
\end{array}\right)\right|=-\frac{m_{S}^{\prime 2} S^{2}}{2}-\frac{m_{h}^{\prime 2} h^{2}}{2}
$$

with $\theta$ equal to

$$
\theta=\frac{1}{2} \arctan \frac{2 \mu v}{\left(m_{h}^{2}-m_{S}^{2}\right)} \approx \frac{\mu v}{m_{h}^{2}} \ll 1 .
$$

This results in the redefinition of the Higgs boson $h \rightarrow h-\theta S$, so the scalar $S$ interacts as the Higgs boson with an additional mixing angle $\theta$. Similarly, because of redefinition of the scalar field, one has $S \rightarrow S+\theta h$. This gives the interaction of new particles with the $\mathrm{SM}$ as

$$
\begin{aligned}
& \mathcal{L}_{S / \chi+S M}=-\theta y_{f} S \bar{f} f-g_{\chi} \theta h \bar{\chi} \chi \\
& +2 \theta \frac{m_{W}^{2}}{v} S W^{+} W^{-}+\theta \frac{m_{Z}^{2}}{v} S Z^{2}+ \\
& +2 \theta \frac{m_{W}^{2}}{v^{2}} S h W^{+} W^{-}+\theta \frac{m_{Z}^{2}}{v^{2}} S h Z^{2}-3 \theta \frac{m_{h}^{2}}{2 v} S h^{2}-\theta \frac{m_{h}^{2}}{2 v^{2}} S h^{3}+\lambda\left(\frac{1}{2} S^{2} h^{2}+v S^{2} h\right) .
\end{aligned}
$$

We left here only terms proportional to the coupling constants $\theta$ and $\lambda$, because we consider that $\theta, \lambda \ll 1$. Boxes indicate interactions that are important for the creation and decay of new particles in experiments. 


\subsection{Dark matter self-interaction}

In our model, the self-interaction of Dark Matter particles results from the process shown in Fig. 5.1. From the structure of this diagram and using dimensional arguments we can say that the cross-section of self-interaction has the form

$$
\sigma_{S I}=\frac{g_{\chi}^{4} m_{\chi}^{2}}{4 \pi m_{S}^{4}} f\left(v_{\chi}, g_{\chi}, \frac{m_{S}}{m_{\chi}}\right),
$$

where $f$ is some dimensionless function of the DM velocity, the mass ratio and, in general, the coupling constant.

Therefore, the result of this section will be a constraint of the couple constant $g_{\chi}$ for each $m_{\chi}$ and $m_{S}$.

It was demonstrated (see e.g. [83]) that for $g_{\chi}^{2} m_{\chi} / m_{S} \ll 4 \pi$ this process is in the perturbative regime and the function $f \approx 1$ for the velocities $v_{\chi} \ll m_{S} / m_{\chi}$. In the nonperturbative regime, the function $f$ can significantly differ from unity and moreover, the function $f$ is not monotonic, can contain resonances [286] and depend on the coupling constant $g_{\chi}$. In this case, it is a bit more difficult to scan over the parameter space of the model and translate the astrophysical information into constraints on the parameters of our model. To avoid this difficulty we calculated the cross-section for a grid of values of constants using the public code DarkSUSY [123]. The dependence on particle velocity implies that we need to average by the velocity distribution of DM particles in a given object (that is used to constrain the cross-section). This operation is implemented in DarkSUSY. Following the logic of the minimal model, we also first assumed for simplicity that DM particles are produced thermally and imposed the corresponding constraints on the coupling constant to guarantee the correct DM abundance. This constraint can be easily imposed with DarkSUSY.

To calculate the function $f$ in Eq. (5.8), we have used VDSIDM model for the scalar mediator from DarkSUSY. We have used an example script VDSIDM_RD.F that calculates the self-interaction cross-section. The constraint of correct DM abundance from thermal production is imposed in this script by default. We scanned the following region of masses: $0.1 \mathrm{GeV} \leq m_{\chi} \leq 5 \mathrm{GeV}$ and $10^{-4} \mathrm{GeV} \leq m_{S} \leq m_{\chi}$. Then, we calculate the cross-section for three average velocities of Dark Matter particles: 10, 200 and $1000 \mathrm{~km} / \mathrm{s}$. Our results demonstrate that for the DM mass in the range from $0.1 \mathrm{GeV}$ to $5 \mathrm{GeV}$ and $m_{S} / m_{\chi}>0.01$ the function $f$ is very close to 1 , see Fig. 5.2, for all values of velocity dispersions that we tried.

From astrophysical observations (see detailed discussion in Section 3 and references 

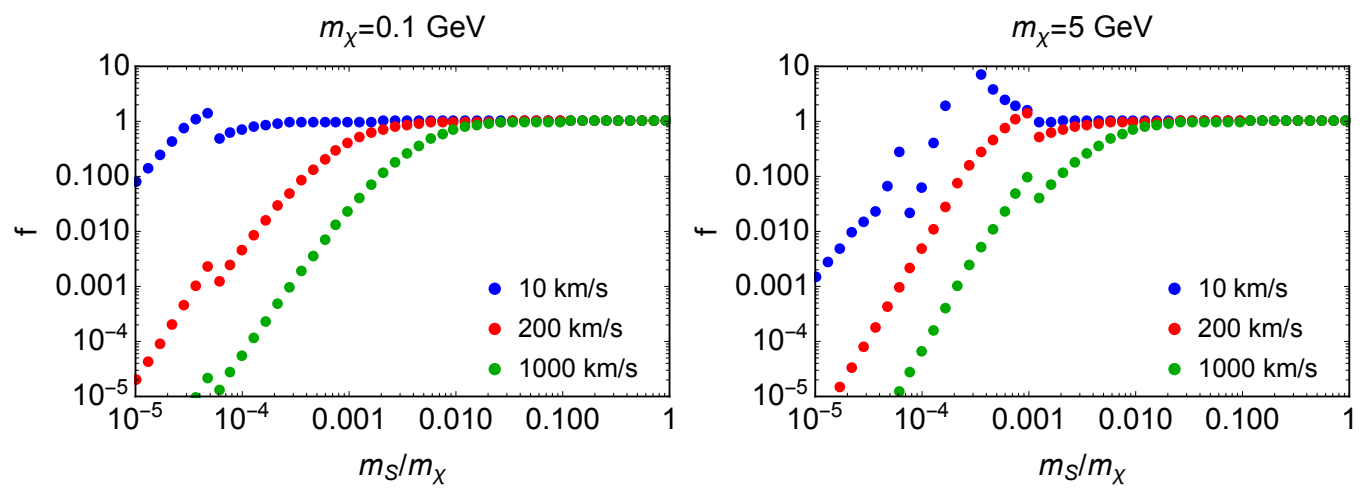

Figure 5.2: Values of the function $f$ from Eq. (5.8) for the DM masses equal to 0.1 and $5 \mathrm{GeV}$ for the coupling constant $g_{\chi}$ that is required by thermal production for the correct DM abundance for three different velocities, 10, 200, and $1000 \mathrm{~km} / \mathrm{s}$. Calculated with DarkSUSY [123].

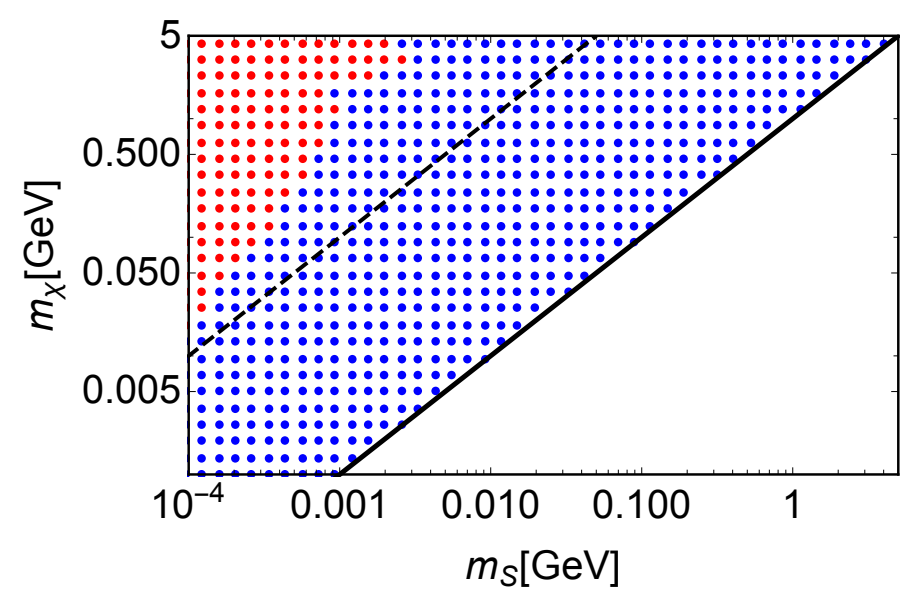

Figure 5.3: Values of the self-interaction cross-section, red points $\sigma_{S I} / m_{\chi}>1 \mathrm{~cm}^{2} / \mathrm{g}$, blue points $\sigma_{S I} / m_{\chi}<1 \mathrm{~cm}^{2} / \mathrm{g}$, for the different values of the DM mass and the scalar mediator. The coupling constant $g_{\chi}$ is chosen from the condition of the correct DM abundance. The region between black solid line and black dashed lines indicates $1>m_{S} / m_{\chi}>0.01$ where we are in the Born regime and $f$ is very close to 1 . We do not consider $m_{S} / m_{\chi}>1$ (to the right of black solid line).

therein), the self-interacting cross-section is bounded as

$$
\sigma_{S I} / m_{\chi}=\frac{g_{\chi}^{4} m_{\chi}}{4 \pi m_{S}^{4}}<1 \mathrm{~cm}^{2} / \mathrm{g},
$$

where $g_{\chi}$ is the value of the coupling constant. In Section 3 we saw that this constraint is valid from dwarf galaxies to galaxy clusters, i.e. for a wide range of velocities of DM particles. In Fig. 5.3 we show that for the chosen parameter space the condition of correct relic abundance for thermally produced DM is stronger than the condition from self-interaction. 

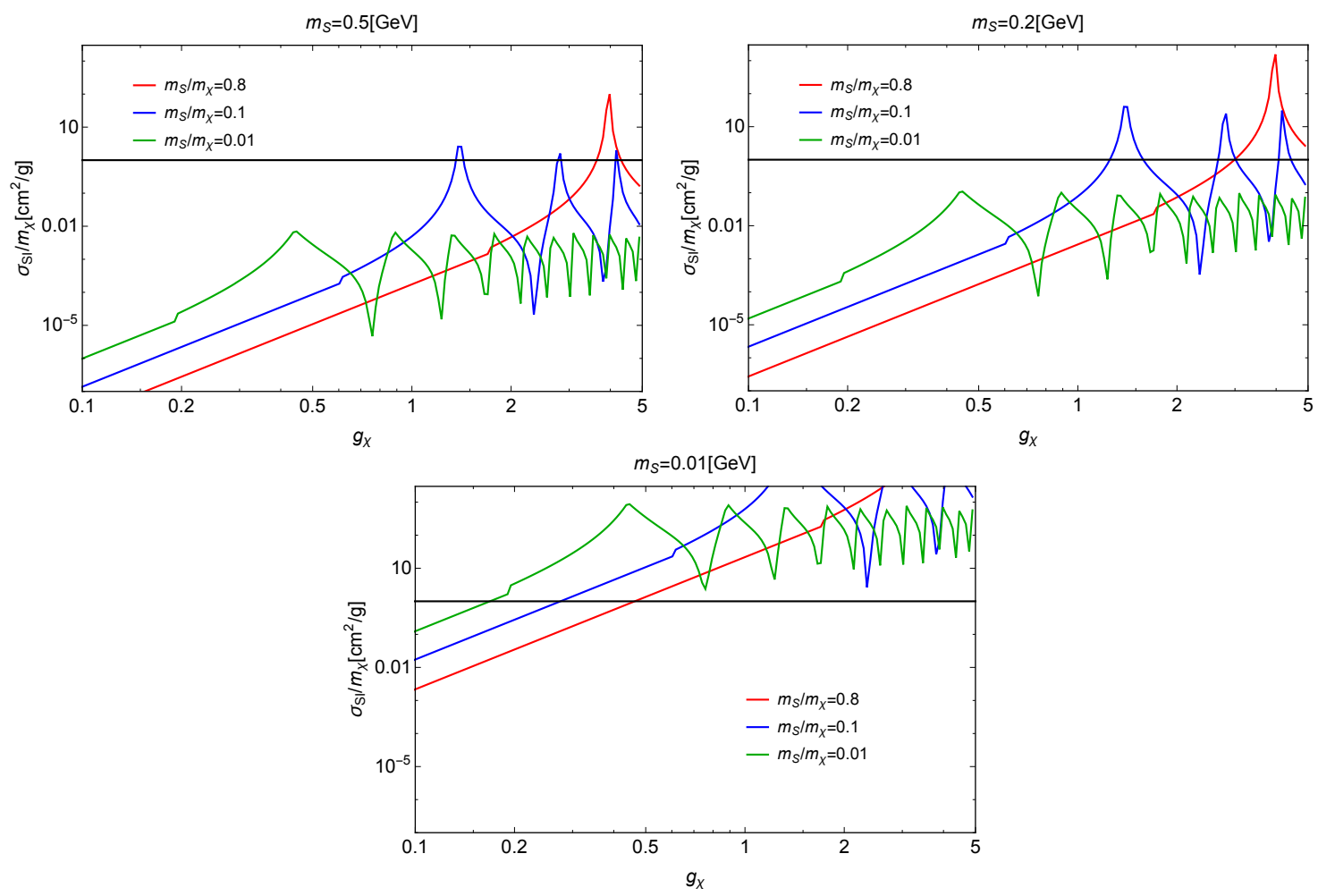

Figure 5.4: The self-interacting cross-section versus the coupling constant $g_{\chi}$ for $v_{\chi}=$ $1000 \mathrm{~km} / s$ with the fixed mass ratio of DM and scalar particles: $m_{S} / m_{\chi}=0.8$ (red line), $m_{S} / m_{\chi}=0.1$ (blue line), and $m_{S} / m_{\chi}=0.01$ (green line). The figures are shown for the scalar masses $m_{S}=0.5 \mathrm{GeV}$ (upper left panel), $m_{S}=0.2 \mathrm{GeV}$ (upper right panel), and $m_{S}=0.01 \mathrm{GeV}$ (bottom panel). The black line represents $\sigma_{S I} / m_{\chi}=1 \mathrm{~cm}^{2} / \mathrm{g}$.

Before we assumed that Dark Matter is produced thermally. For example, if there exists some new physics beyond the model (5.1) there may be other mechanisms of the DM production and our coupling constant $g_{\chi}$ again becomes a free parameter. In this case, we cannot use the condition for the correct DM abundance and the conservative limit can be derived from the constraint on the self-interaction cross-section (5.9). To do so, we used model VDSIDM in the DarkSUSY package to calculate the self-interaction crosssection as function of interaction strength $g_{\chi}$, for each pair of values of masses $m_{S}$ and $m_{\chi}$. The cross-section may depend also on the velocity of DM particles and we made the calculation for the value $v_{\chi}=1000 \mathrm{~km} / \mathrm{s}$, typical for galaxy clusters.

The examples of the results are shown in Fig. 5.4. For each pair of values of $m_{S}$ and $m_{\chi}$ the dependence on the coupling constant $g_{\chi}$ is non-trivial and has resonances for large values of $g_{\chi}$. For every $m_{S}$ and $m_{\chi}$ we need to find the range of values of $g_{\chi}$ where $\sigma_{S I} / m_{\chi}<1 \mathrm{~cm}^{2} / \mathrm{g}$ despite resonances.

The dependence of $\sigma_{S I} / m_{\chi}$ on the mass $m_{S}$ is quite simple and results in the overall scaling of self-interaction cross-section with $m_{S}$. Namely, lower $m_{S}$ gives larger values 


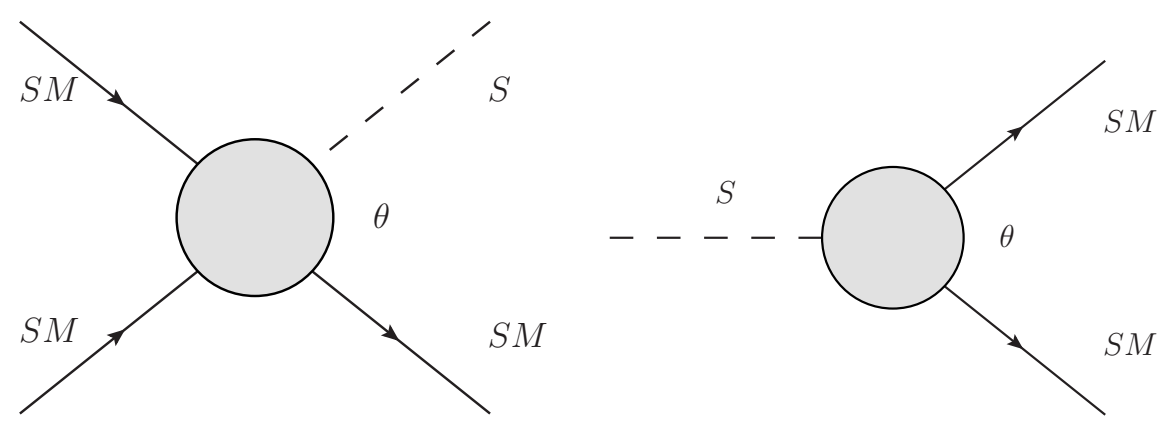

Figure 5.5: Left panel: A schematic diagram of the production of a scalar particle $S$ at colliders. Right panel: A schematic diagram of the decay of a scalar particle $S$ into SM particles.

of self-interaction cross-section for the same coupling constant but does not change the shape of the function $\sigma_{S I} / m_{\chi}\left(g_{\chi}\right)$.

To put a constraint on $g_{\chi}$, we will demand that the whole region with resonances is above $\sigma_{S I} / m_{\chi}<1 \mathrm{~cm}^{2} / \mathrm{g}$. In Fig. 5.4 we see that for $m_{S} / m_{\chi}=0.8$ the constraint on $g_{\chi}$ from self-interaction exists for $m_{S}<0.2 \mathrm{GeV}$, while for $m_{S} / m_{\chi}=0.1$ and $m_{S} / m_{\chi}=$ 0.01 we can use the same condition $m_{S}<0.01 \mathrm{GeV}$. In the analysis below we choose these mass ratios as an example.

\subsection{Constraints from accelerator searches}

At accelerators, one can search for the mediator $S$ that can be detected via decay products of this particle into SM particles or as a missing energy and a missing transverse momentum $p_{T}$, see Fig. 5.5. In the case of missing energy (momentum), the number of events will be proportional to $\theta^{2}$ while for the detection of scalar decay products the number of events is proportional to $\theta^{4}$ [287-289]. Although $\theta \ll 1$ and consequently $\theta^{4} \ll \theta^{2}$, it is not obvious a priory what type of experiments gives stronger constraints as visible decay may be much easier to detect against backgrounds (some of the $\theta^{4}$ experiments even aim at background-free regime). Below we largely follow paper III [31].

Accelerator searches discussed in this section provide constraints on $\theta$ as a function of $m_{S}$.

\subsubsection{Accelerator phenomenology of the scalar portal}

Before describing the constraints on the scalar portal from various types of experiments, let us briefly review the main production and decay channels of light scalars that may be relevant at accelerators. Phenomenology of the scalar portal at accelerators was recently 
reviewed in paper III [31]. Production of scalars goes both through the mixing with the Higgs boson and trough the so-called "quartic coupling" $\lambda$, see Eq. (5.1), while the decay of scalars is possible only through the mixing with Higgs bosons. Depending on the energy of a beam, different production channels play more or less an important role. For example, for the energy of the proton beam $120 \mathrm{GeV}$ (that corresponds to the DUNE experiment [290]), direct production of scalars via proton bremsstrahlung and Deep Inelastic Scattering (DIS) gives the main contribution, see Fig. 5.6. For energies from $400 \mathrm{GeV}$, corresponding e.g. to CHARM [287], SHiP [291] (see below) or the LHC, the main production channel is from decays of kaons and $B$ mesons, see Table 5.1 with the main production channels. The production from quartic coupling is important for small values of the mixing angle $\theta \lesssim 10^{-9}$, so it can modify the lower bound of the sensitivity curves. Also, scalars can be produced through quartic coupling in Higgs boson decays, which is an important production channel for the LHC.

The main decay channels of scalars are decays into pairs of leptons, into a pair of photons or into hadrons. In the literature there is a well-known theoretical uncertainty in the decay widths of the channels $S \rightarrow 2 \pi$ and $S \rightarrow 2 K$ [292-294]. For these decay channels, we use the approach from [293]. Branching ratios and a corresponding lifetime of the scalar are given in Fig. 5.7.

This summary of accelerator phenomenology of the scalar portal allows us to interpret experimental results in terms of constraints on our model and discuss sensitivities of future experiments. We describe this results below.

\subsubsection{Experiments constraining scalar portal}

Let us consider the experiments that give constraints on the visible decay of a light scalar into SM particles. One relevant type of experiments is a fixed target or "beam dump" experiments with intense proton beams. The general idea is the following. To create a feebly interacting particle we need to maximize the number of events. This is done by heating a target with an intense proton beam. Most of the products of collisions are Standard Model particles that should be removed. This is achieved by placing a decay volume and detectors far from the target and additionally screening them from the SM particles, such that only a rare long-lived particle can reach the decay volume. The details of the sensitivity curve of a given experiment are defined by the geometry of the experiment and parameters of the beam. Below we describe two past and one proposed experiments of this type.

CHARM [287] is a fixed target experiment that used a $400 \mathrm{GeV}$ proton beam dumped into a thick copper target. If a long-lived particle is created in such collisions it can be detected if it visibly decays inside a $35 \mathrm{~m}$ decay volume placed $480 \mathrm{~m}$ from the target. The main production channel of light scalars in this experiment was decay of kaons $K^{ \pm / 0} \rightarrow$ $\pi^{ \pm / 0}+S$, which defines the maximal mass of the scalar that can be probed $m_{S}<m_{K}-$ $m_{\pi} \sim 350 \mathrm{MeV}$. The number of kaons in CHARM was $N_{K} \approx 1.4 \times 10^{16}$. 


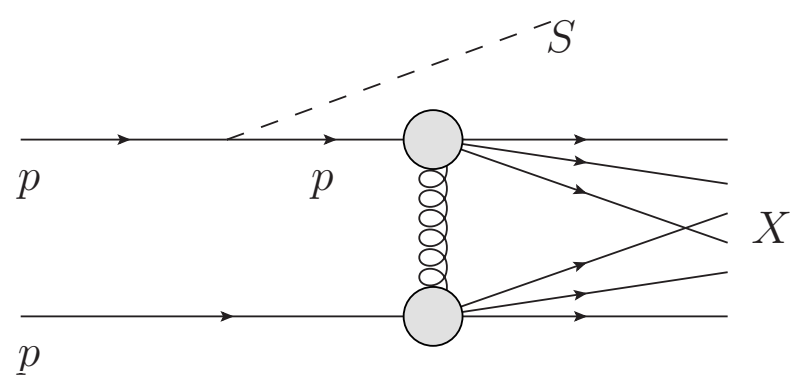

(a)

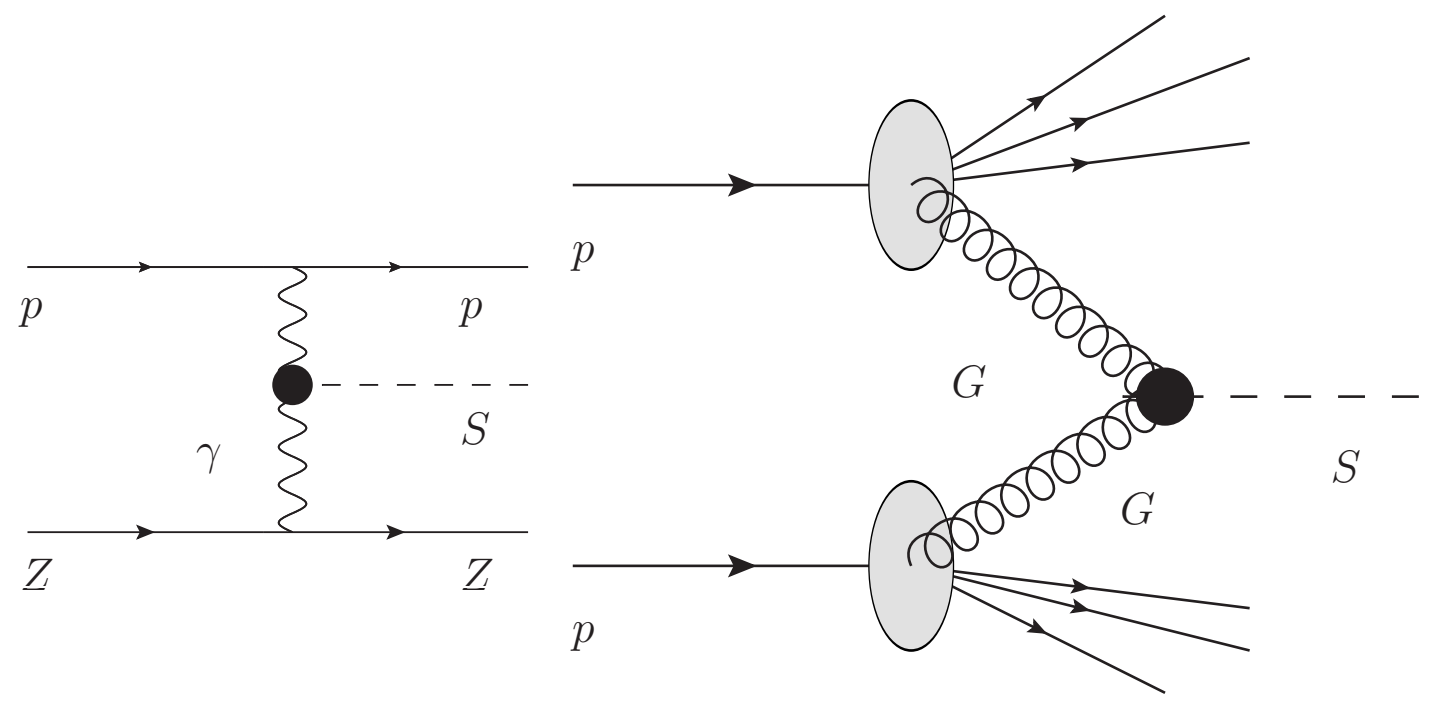

(b)

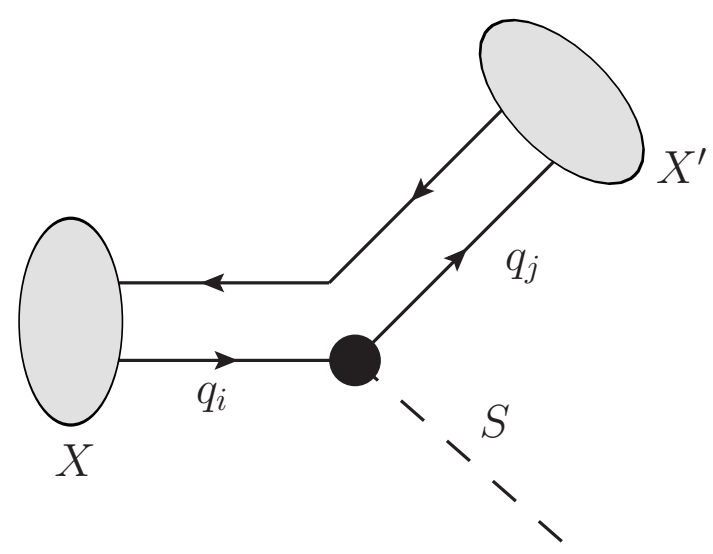

(c)

Figure 5.6: Examples of diagrams for the main channels of the production of light scalars in proton-nucleus collisions: proton bremsstrahlung (a), photon and gluon fusion (b), decay of secondary mesons (c). Taken from paper III [31]. 


\begin{tabular}{|l|c|c|}
\hline Process & $\mathrm{BR}\left(m_{S}=0\right) / \theta^{2}$ & Closing mass [GeV] \\
\hline$K^{ \pm} \rightarrow S \pi^{ \pm}$ & $1.7 \cdot 10^{-3}$ & 0.350 \\
\hline$K_{L}^{0} \rightarrow S \pi^{0}$ & $7 \cdot 10^{-3}$ & 0.360 \\
\hline$K_{S}^{0} \rightarrow S \pi^{0}$ & $1.2 \cdot 10^{-5}$ & 0.360 \\
\hline$B^{ \pm} \rightarrow S K_{1}^{ \pm}(1270)$ & $\left(9.1_{-4.0}^{+3.6}\right) \cdot 10^{-1}$ & 3.82 \\
\hline$B^{ \pm} \rightarrow S K_{0}^{*, \pm}(700)$ & $7.6 \cdot 10^{-1}$ & 4.27 \\
\hline$B^{ \pm} \rightarrow S K^{*, \pm}(892)$ & $\left(4.7_{-0.8}^{+0.9}\right) \cdot 10^{-1}$ & 4.29 \\
\hline$B^{ \pm} \rightarrow S K^{ \pm}$ & $\left(4.3_{-1.0}^{+1.1}\right) \cdot 10^{-1}$ & 4.79 \\
\hline$B^{ \pm} \rightarrow S K_{2}^{*, \pm}(1430)$ & $3.0 \cdot 10^{-1}$ & 2.56 \\
\hline$B^{ \pm} \rightarrow S K^{*, \pm}(1410)$ & $\left(2.1_{-1.1}^{+0.6}\right) \cdot 10^{-1}$ & 3.57 \\
\hline$B^{ \pm} \rightarrow S K^{* \pm}(1680)$ & $\left(1.3_{-0.4}^{+0.5}\right) \cdot 10^{-1}$ & 3.26 \\
\hline$B^{ \pm} \rightarrow S K_{0}^{*, \pm}(1430)$ & $8.1 \cdot 10^{-2}$ & 3.82 \\
\hline$B^{ \pm} \rightarrow S K_{1}^{ \pm}(1400)$ & $\left(1.6_{-1.1}^{+0.6}\right) \cdot 10^{-2}$ & 2.28 \\
\hline$B^{ \pm} \rightarrow S \pi^{ \pm}$ & $\left(1.3_{-0.3}^{+0.3}\right) \cdot 10^{-2}$ & 5.14 \\
\hline
\end{tabular}

Table 5.1: Properties of the main channels of production of a scalar $S$ from decays of kaons and $B$ mesons through the mixing with the Higgs boson. First column: decay channels; Second column: branching ratios of 2-body meson decays evaluated at $m_{S}=0$ and normalized by $\theta^{2}$. For $B$ mesons the numerical values are given for $B^{ \pm}$mesons; in the case of $B^{0}$ meson all the given branching ratios should be multiplied by a factor of 0.93 that comes from the difference in total decay widths of $B^{ \pm}$and $B^{0}$ mesons [14]. Uncertainties (where available) follow from uncertainties in meson transition form-factors; Third column: effective closing mass, i.e. a mass of a scalar at which the branching ratio of the channel decreases by a factor of 10 . Taken from paper III [31].
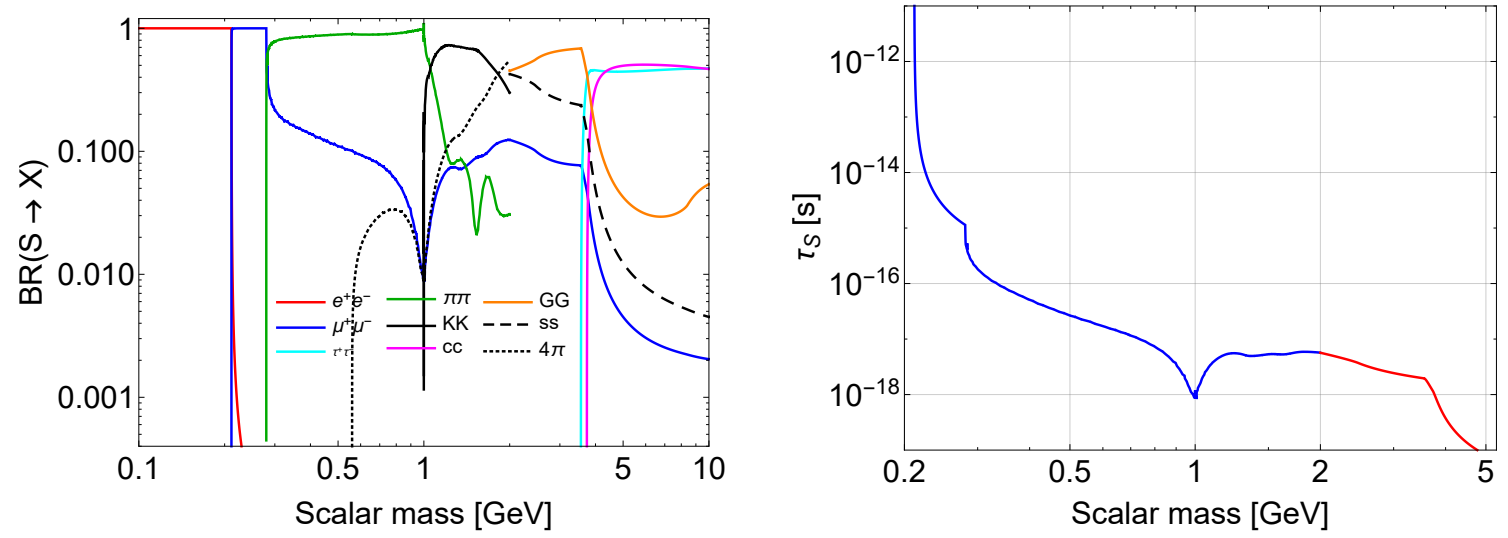

Figure 5.7: Left panel: Branching ratios of decays of a scalar $S$ as a function of its mass. Right panel: The lifetime of a scalar $S$ as a function of its mass with the mixing angle $\theta^{2}=1$. Solid blue line denotes the lifetime calculated using decays into leptons, kaons, and pions, while solid red line - the lifetime obtained using decays into quarks and gluons within the framework of perturbative QCD. Taken from paper III [31]. 


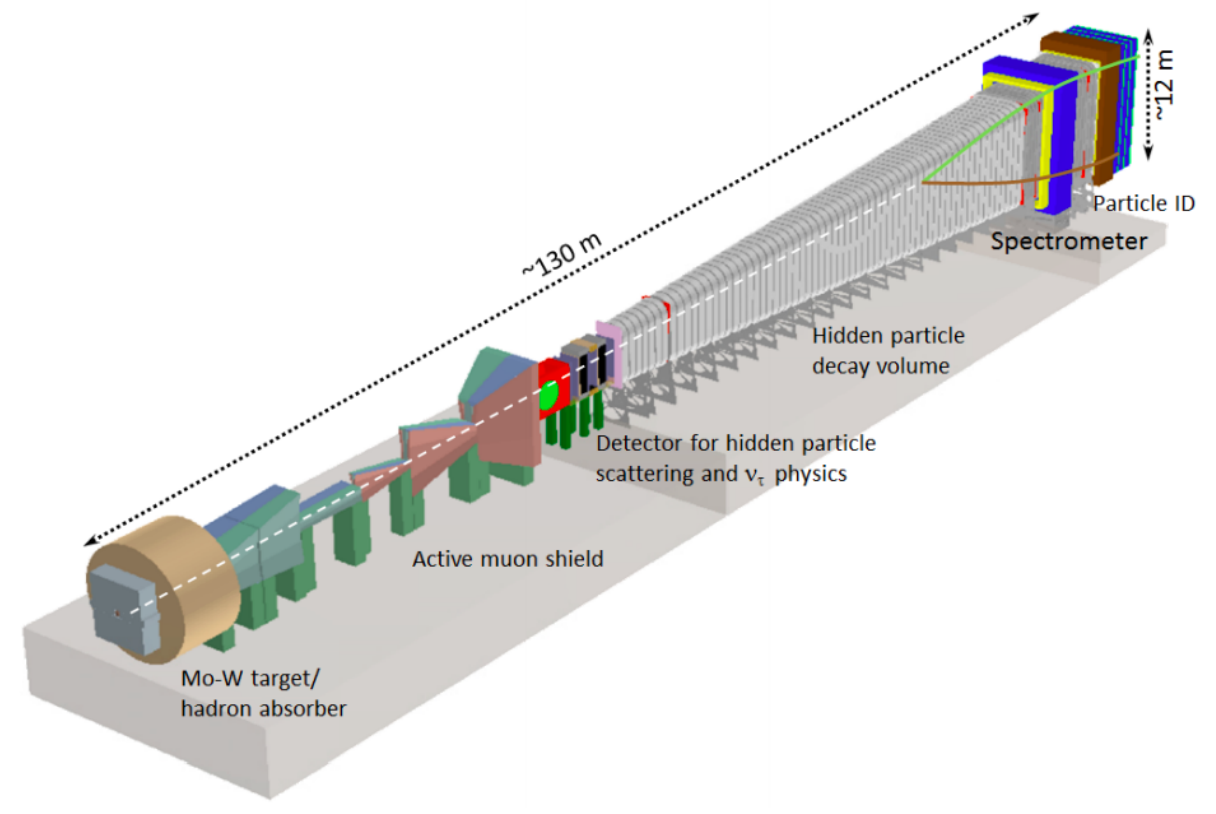

Figure 5.8: The scheme of the SHiP experiment. Taken from [283].

$\mathrm{KTeV}$ [288] is also a beam dump experiment that utilized a Tevatron ring, using a proton with $800-\mathrm{GeV}$ beam energy. The products of a proton beam are cleaned with magnets and absorbers in such a way that only a neutral kaons beam remain. $\mathrm{KTeV}$ had a $65 \mathrm{~m}$ long vacuum decay volume, where decays of $K_{L} \mathrm{~s}$ were measured. This experiment put an upper bound of the decays $\operatorname{BR}\left(K_{L} \rightarrow \pi \mu^{+} \mu^{-}\right)<3.8 \times 10^{-10}$ [288] and $\operatorname{BR}\left(K_{L} \rightarrow\right.$ $\left.\pi e^{+} e^{-}\right)<2.8 \times 10^{-10}$ [295], which in turn puts a constraint on the scalar because of the reaction $K_{L} \rightarrow \pi^{0} S\left(\ell^{+} \ell^{-}\right)$.

Even small mixing angles can be probed with the next-generation Intensity Frontier experiments (e.g. SHiP [296], MATHUSLA [284], FASER [297], see also [298] for a review). Below we use SHiP, currently the most developed among these proposals, as an example.

SHiP is a fixed-target experiment that is proposed to build at CERN [296]. The proton beam will be taken from SPS with the energy $400 \mathrm{GeV}$. It is planned that after 5 years of operation SHiP will deliver $2 \cdot 10^{20}$ protons-on-target. The design of the experiment is illustrated in Fig. 5.8. Protons hit the target and create a large number of SM particles, first of all mesons that later decay. The Standard Model particles are either stopped by hadron absorber or are deflected by the active muon shield. The goal is to create a backgroundfree experiment which means that no SM particles should reach the decay volume. If a new feebly interacting particle was created e.g. in the decay of mesons, it will reach the decay volume. SHiP is sensitive to particles that are long-lived enough not to decay on their way to the decay volume $(\sim 50 \mathrm{~m})$ and still can decay into SM particles inside it 


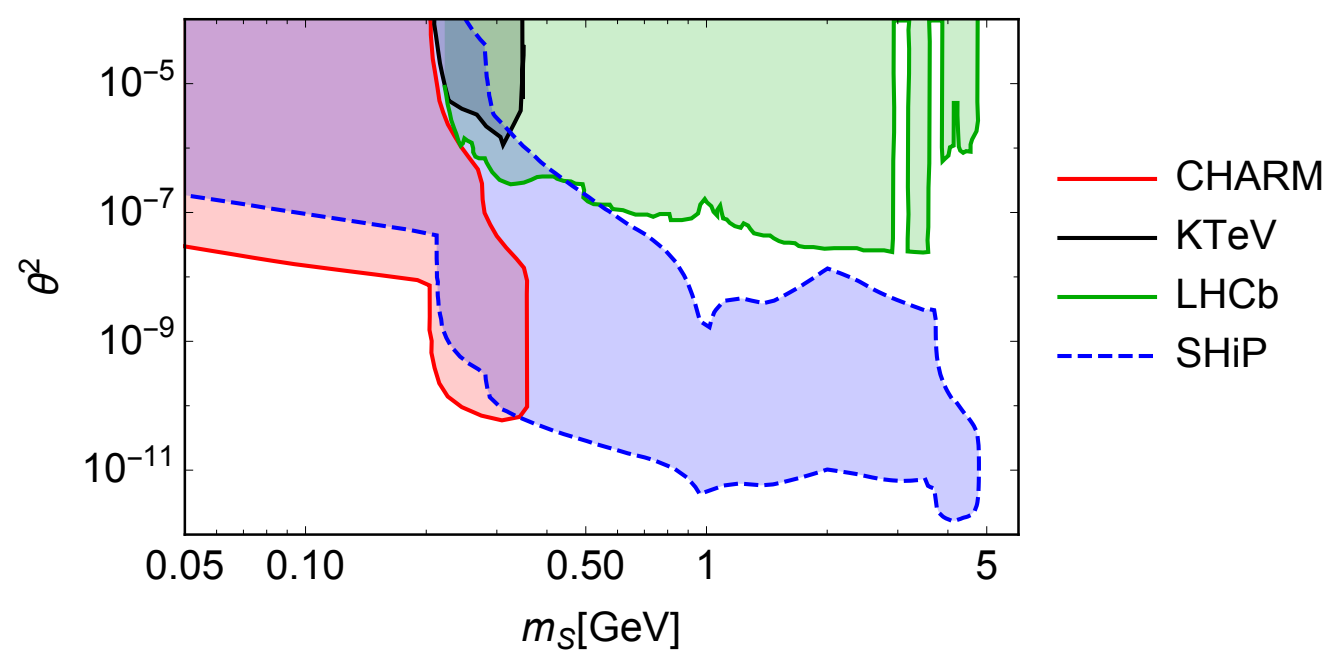

Figure 5.9: Constraints on the mixing angle between the scalar $S$ and the Higgs boson from CHARM [287], KTeV [288], LHCb [289] experiments and the expected sensitivity of SHiP [291] (obtained using the results of section 5.4.1).

(another $\sim 50 \mathrm{~m}$ ). The decay products will be detected by trackers, calorimeter and other detectors. The sensitivity of SHiP for the scalar portal will be reported in the collaboration paper [299]. Here we use the estimate from paper III [31].

Another kind of constraints comes from LHC experiments. Unlike beam dump experiments, the relevant searches at these type of experiments are not background-free and therefore one has to suppress the SM background in order to observe new physics. For example, at the LHCb experiment at CERN [289] the decays of a light scalar can be distinguished from the SM background by using the rare decay $B^{+} \rightarrow K^{+} S\left(S \rightarrow \mu^{+} \mu^{-}\right)$ that is strongly suppressed in the Standard Model. This gives constraints on the light scalar for the mass range $250<m_{S}<4700 \mathrm{MeV}$ and lifetimes $0.1<\tau_{S}<1000$ ps for the data from 7 and $8 \mathrm{TeV}$ runs with total luminosity $3 \mathrm{fb}^{-1}$. Therefore, a constraint on the branching ratio of this channel from $10^{-7}$ up to $10^{-10}$, depending on the scalar lifetime.

The constraints on $\theta$ are presented in Fig. 5.9. Further improvement of the constraints on $\theta$ is expected from high-luminosity LHC runs and from Belle II [300] that recently started to collect data. Smaller angles can probably be probed by the searches for displaced vertices, see e.g. [301] and references therein.

\subsubsection{Constraints from the invisible Higgs decay}

The production of DM at accelerators is schematically shown in Fig. 5.10. The invisible Higgs decay gives the strongest constraints and was searched at CMS [302] using missing transverse momentum signature. In this process, the production of DM is enhanced if the 


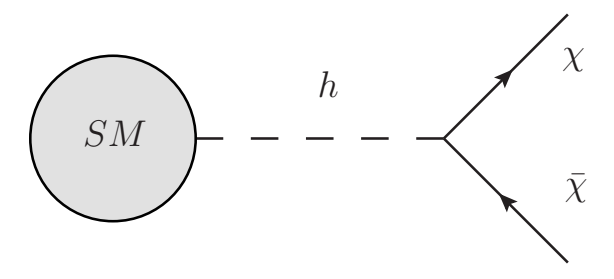

Figure 5.10: Feynman diagram for the Higgs boson decay into DM particles for the model (5.1).

Higgs boson is a real particle. The $2 \sigma$ upper bound was found to be

$$
\mathrm{BR}(h \rightarrow \text { inv. })<0.19 .
$$

In our model the Higgs boson interacts with a DM particle, see the second boxed term in Eq. (5.7). It gives the same invisible Higgs decay signature with a branching ratio

$$
\mathrm{BR}(h \rightarrow \bar{\chi} \chi)=\frac{\theta^{2} g_{\chi}^{2} m_{h}}{8 \pi \Gamma_{h}},
$$

where $m_{h} \approx 125 \mathrm{GeV}$ [14] and $\Gamma_{h} \approx 4.1 \mathrm{MeV}$ [303] are Higgs boson's mass and total decay width correspondingly. Experimental measurement of the Higgs boson invisible decay width puts a bound

$$
\theta^{2} g_{\chi}^{2}<1.6 \cdot 10^{-4}
$$

\subsection{Direct detection}

As discussed in Section 2, direct detection experiments aim to detect DM matter particles from the Milky Way halo that fly around (and through) Earth. This is done using the process schematically presented in Fig. 5.11.

The results of direct detection experiments are often presented in terms of constraints on $\sigma_{p}$, the total cross-section of the free nucleon-DM scattering, i.e. the total cross-section for the same diagram 5.11 but in a theory with the effective Lagrangian [304]

$$
\mathcal{L}_{\chi p}=c_{p} \bar{\chi} \chi \bar{p} p
$$

where $p$ is a nucleon, i.e. proton or neutron, we will use proton as an example. This gives the cross-section

$$
\sigma_{p}=\frac{c_{p}^{2} \mu_{p}^{2}}{\pi}
$$




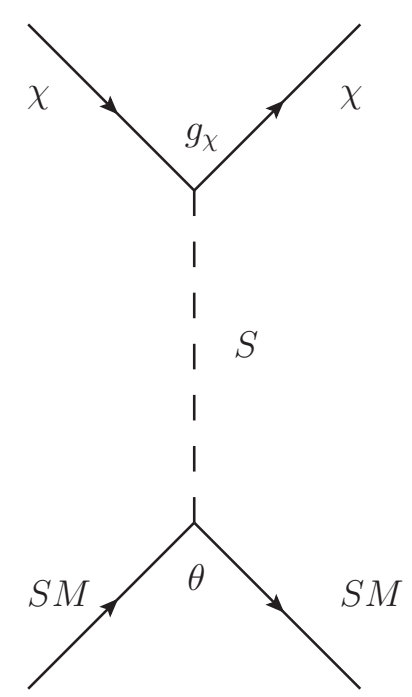

Figure 5.11: A schematic Feynman diagram for the direct detection searches.

where $\mu_{p}$ is the reduced mass of DM and a proton

$$
\mu_{p}=\frac{m_{p} m_{\chi}}{m_{p}+m_{\chi}}
$$

The quantity $\sigma_{p}$ is defined in such a way that it does not depend on the type of nuclei used in an experiment, the minimal measured recoil energy, and other experiment-dependent details. It makes it convenient for the comparison between different experiments. When $\sigma_{p}$ is derived from the direct experimental data it is assumed (in terms of our simple model) that the scalar mediator mass $m_{S}>5 \cdot 10^{-3} m_{\chi}$ and we can neglect the velocity dependence in an effective scalar-proton interaction [305]. We also consider here spin-independent scattering.

Under this assumptions, the coupling constant in the effective Lagrangian (5.13) should be chosen as [304]

$$
c_{p}=\frac{\sqrt{2} \theta g_{\chi} m_{p}}{v m_{S}^{2}}\left(\sum_{q=u, d, s} f_{q}+\frac{2}{9} f_{G}\right) .
$$

Here $v$ is the Higgs field vacuum expectation value, and the constants $f$ correspond to the quark content in a proton, $f_{u}=0.02, f_{d}=0.026, f_{s}=0.118$, and $f_{G}=1-\sum_{q=u, d, s} f_{q}$. Using it we can compute the total DM-proton cross-section [285],

$$
\sigma_{p}=\frac{c_{p}^{2} \mu_{p}^{2}}{\pi} \approx 4.4 \cdot 10^{-34} \mathrm{~cm}^{2} \theta^{2} g_{\chi}^{2}\left(\frac{1 \mathrm{GeV}}{m_{S}}\right)^{4}\left(\frac{m_{\chi}}{1 \mathrm{GeV}}\right)^{2}\left(1+\frac{m_{\chi}}{m_{p}}\right)^{-2} .
$$

As we saw in Section 5.4, for searches for the invisible Higgs decay the constraint has exactly the same combination of coupling constants $\theta^{2} g_{\chi}^{2}$ that enters in Eq. (5.17) for direct 

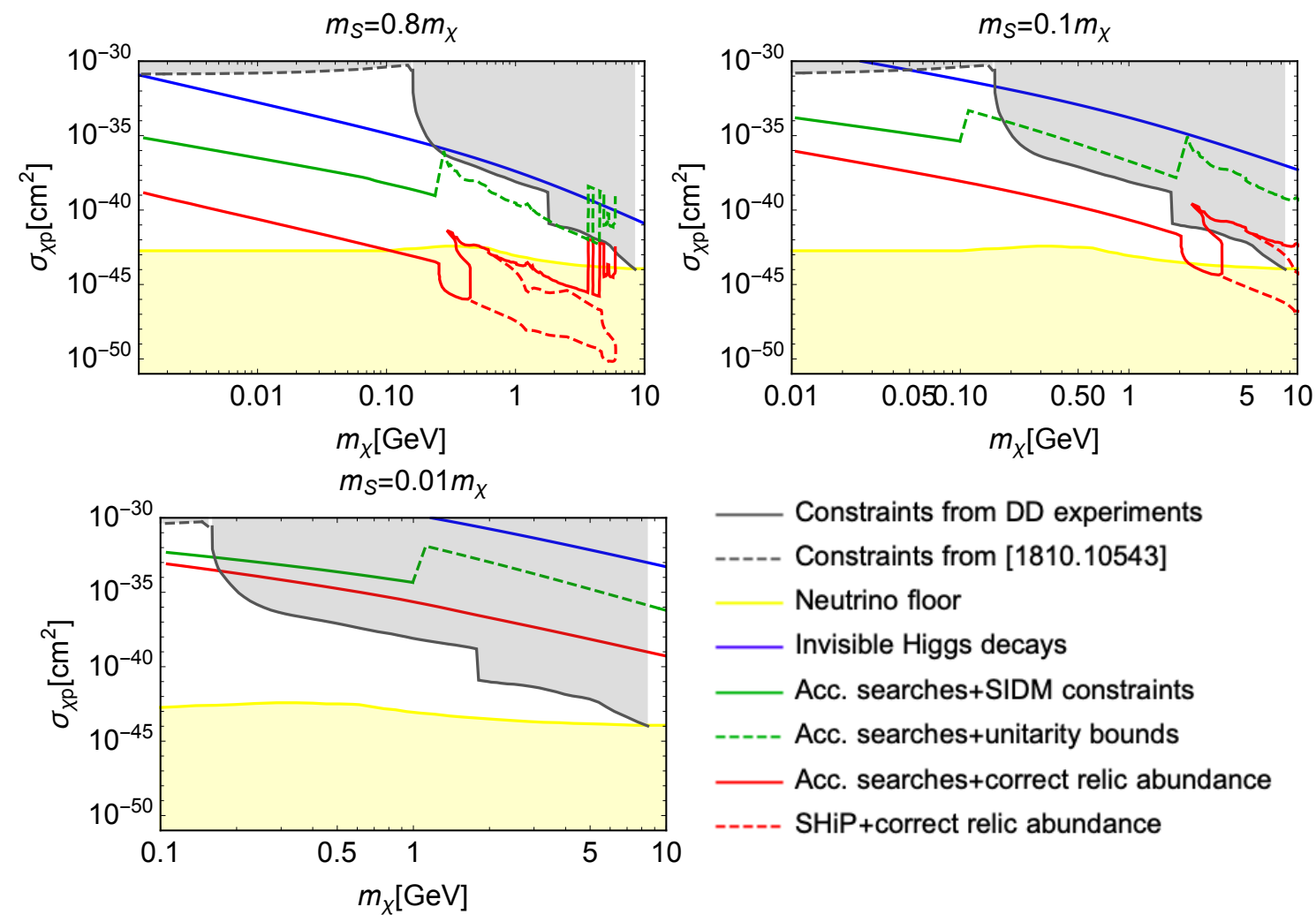

Figure 5.12: Constraints on the direct detection cross-section from invisible Higgs decay (blue solid line), from the combination of the correct relic abundance and accelerator searches (red solid line) or the future planned intensity frontier experiment SHiP (red dashed line), and from the combination of accelerator searches and the constraint on the self-interaction cross-section (green solid line) for the different ratios of masses between the scalar mediator and DM. Solid and dotted gray lines are limits for direct detection experiments [159, 276, 306-308]. The yellow region is a so-called "neutrino-floor", an irreducible background for the direct detection experiments [306], that we add as a benchmark.

detection. Using these constraints (5.12) we get

$$
\sigma_{\chi p}^{\text {inv. } \mathrm{h}} \lesssim 7.0 \cdot 10^{-38} \mathrm{~cm}^{2}\left(\frac{1 \mathrm{GeV}}{m_{S}}\right)^{4}\left(\frac{m_{\chi}}{1 \mathrm{GeV}}\right)^{2}\left(1+\frac{m_{\chi}}{m_{p}}\right)^{-2} .
$$

We can also use the constraints from accelerator searches for a light scalar (see Fig 5.9). However, these constraints include only the mixing angle $\theta$ as a function of the scalar mass and not the coupling constant $g_{\chi}$ that also enters into the expression for $\sigma_{p}$. To proceed we have two options. One possibility is to use the constraints from the self-interaction (5.9) that limit $g_{\chi}$ from above. Another option is to constrain $g_{\chi}$ using the condition that the correct DM abundance should be produced (assuming certain production mechanism). We 
will consider both possibilities, assuming thermal production as a minimal model. In this section, we assumed that the ratio of the masses of the scalar mediator and the DM fermion is $1>m_{S} / m_{\chi}>0.01$, to guarantee that self-scattering of DM is in the perturbative regime and the simple constraint (5.9) holds. We remind that for this region of parameters the condition on $g_{\chi}$ from the correct relic abundance with thermal production is stronger than from self-interaction, see Fig. 5.3.

These constraints (from the invisible Higgs decay and from accelerator searches for the light scalar assuming thermal production) are presented in Fig. 5.12. For this plot to be two-dimensional, we assume a fixed ratio of the masses $m_{S}=\epsilon m_{\chi}$ and show the constraints for different values of $\epsilon<1$. We see that the combination of accelerator searches and cosmological bounds gives a constraint that is stronger than the constraint from invisible Higgs decays for almost all points in the probed parameter space.

\subsection{Conclusions}

In this section, we demonstrated how different constraints on Dark Matter particles can be combined together for a specific particle physics model. In particular, we derived predictions for Dark Matter direct detection experiments from accelerator constraints on the light scalar (that mediates in our model the interaction between DM and the Standard Model) with the requirement of the correct abundance of Dark Matter or with astrophysical constraints on Dark Matter self-interaction. For our model, the bounds from the fixed target experiments (CHARM, KTeV) and LHCb combined with astrophysical or cosmological constraints are stronger than other constraints (see e.g. [276]) in a significant part of the parameter space. The bounds from invisible Higgs decays are somewhat weaker, but are still competitive with other constraints and can be applied directly, without additional information from cosmology and astrophysics.

In this section, we considered only the simplest Dark Matter model of this kind. Namely, we assumed a scalar mediator, thermally produced Dark Matter, and we considered values of the ratio of the Dark Matter and mediator masses for which Dark Matter self-interaction is in the perturbed regime. We plan to perform the full exploration of the parameter space and consider a more general class of models elsewhere. 


\section{Chapter 6}

\section{Conclusions and outlook}

This thesis is devoted to the astro-particle approach to the problem of Dark Matter. We started by presenting the evidence that makes us believe that the dynamics of most of the galaxies of various sizes as well as clusters of galaxies and of the Universe as a whole cannot be described by the Standard Model of particle physics supplemented with General Relativity. We have also reviewed the arguments suggesting that these phenomena are probably explained by the existence of a new particle and concentrated our attention on this hypothesis. If true, this means that the resolution of the DM puzzle is not only very important for astrophysics and cosmology, but would play also a profound role for the future development of particle physics.

Particle physics is at the crossroads at the moment - its Standard Model has survived an impressive number of experimental checks, consistency checks, and precision tests. Since the discovery of its last predicted particle - the Higgs boson - LHC, the flagship of modern particle physics has measured with high significance all of its main creation and decay channels, checked that their branching rations and other relative characteristics are consistent with the Standard Model. Many non-trivial predictions of the electroweak theory, including three and four vector boson processes, were tested beyond the leading order, many rare processes predicted by the Standard Model have been observed (including also light on light scattering).

This spectacular success of the Standard Model emphasizes even more that at the moment we are to a large extent agnostic about the direction in which the Standard Model must be extended to accommodate experimentally confirmed mysteries that it can not explain - the origin of neutrino masses, the nature of Dark Matter and the mechanism for generation of the asymmetry between matter and anti-matter. There are many mechanisms and extensions of the Standard Model that have been proposed to address these questions. They predict the existence of new particles and fields of various types, with very different properties. Correspondingly, experimental strategy to discover them should also be very different. In particular, we do not even know if the new particles have not been discovered yet because they are too heavy or because their interaction is too weak. These options imply experiments of two different types - the so-called "energy frontier" allowing to create and detect heavier and heavier particles, and "intensity frontier" maximizing the number 
of events and allowing to discover new particles that are extremely rare.

Shedding light on any of the above-mentioned "beyond Standard Model problems" or even constraining possible resolutions may give us an idea where to look for new particle physics and how to optimize our experimental strategy for the coming decades.

Among Beyond the Standard Model questions, the problem of Dark Matter plays a special role. Indeed, as this problem comes from astrophysics and cosmology, it naturally brings to physics Beyond the Standard Model an enormous amount of observational data that is complementary to particle physics data. Of course, the data originating from astronomical observations are very different from particle physics data. In astronomy we have much less control of the observed objects as they are located very far from us, the number of directly measured observable is very limited, most of the relevant quantities have to be derived. This, of course, introduces systematic uncertainties that are typically much less controlled than in particle physics.

On the other hand, the number of different independent observations is very large combining many different observations provides an efficient method to marginalize over unknown parameters and reduce both theoretical and observational uncertainties. The conditions that we can observe in the Universe are impossible to reproduce on Earth. Additionally, the bounds that are obtained from astronomical data are often to a large extent model independent. For example, confirming that Dark Matter is warm, cold or self-interacting (see Sections 2 and 3 for definitions and details) or even detecting a signal from decay or annihilation of DM particles in the space would not be enough to fix one single model of Dark Matter. However, it would challenge many models and in this way disfavor whole directions of development of particle physics, both on its theoretical and, even more importantly, experimental sides.

In this thesis, we described three different examples of constraining the properties of DM particles. Section 3 was devoted to self-interacting Dark Matter. In this section, we have reviewed the constraints on the cross-section of dark-matter self-interaction imposed by the observed properties of dark-matter dominated objects. We have collected ensembles of haloes of all observed sizes simulated for SIDM. These simulations were performed including many effects of baryonic matter that can affect the properties of DM halos and observable quantities. Analyzing these simulations we were able to make robust predictions that can be directly compared with observations. On the observational side, we used the stars' velocity dispersion data for the dwarf spheroidal galaxies, the rotation velocity data for spiral galaxies and weak lensing data for galaxy clusters. We have demonstrated that it is possible to compare these data with SIDM simulations without fitting to parametric models of the DM density distribution, but rather using the estimates of the total mass that may be derived from the observational data more directly. We believe that the analysis presented here demonstrates how to obtain robust constraints on SIDM despite all observational uncertainties and theoretical difficulties. We have also observed that realistic modeling of baryonic effects in simulations is crucial for constraining SIDM, especially if 
we want to include the data from spiral galaxies. In our work, we used Eagle to describe baryonic effects. It would be extremely important to use an alternative framework to account for baryonic effects. We also need to perform simulations for other cross-sections. On the observational side, it is very important to add also X-ray and strong lensing data for the case of galaxy clusters. For the case of dwarf galaxies, very significant progress can be achieved when the data on 3D velocities of stars will become available, from Gaia or elsewhere (see e.g. [309] for a recent review). Another interesting direction is to add to the analysis the possibility of late kinetic decoupling and the constraints from Ly-alpha forest [310].

Section 4 was devoted to another approach to uncover the nature of Dark Matter indirect detection. Here our main interest was related to a new major experimental facility that is expected to start operating in the next several years and could become the real game changer in the field of $\gamma$-ray astronomy and, in particular, in DM indirect detection - the Cherenkov Telescope Array. This new IACT will be working in the energy range $20 \mathrm{GeV}$ - $300 \mathrm{TeV}$ and is expected to be much more powerful than existing $\gamma$-ray telescopes.

We have tested the software package ctools adopted by the CTA collaboration for the detection of a possible DM annihilation signal. Starting from version 1.6.0-dev1, ctools is ready for such an analysis. We discussed here only the signal from the prompt emission of photons. It is potentially interesting to include in the analysis a possible contribution from the Inverse Compton effect, having different spatial morphology and spectral properties. Although the treatment of systematic errors is not yet implemented in ctools, it is possible to take them into account adopting a simple physical approach that does not require any modifications of the code. This method, based on a limited number of counts in each energy bins, suggests that for long exposure observations of the Galactic Center it can make sense to use the LSTs only for less than 50 hours (even if the whole observation may be much longer). Indeed, the number of counts in the energy bins below $150 \mathrm{GeV}$, where the LSTs operate, is huge and the data will quickly become dominated by systematics, even if it is at the very optimistic level of $1 \%$. This may potentially free a significant amount of the LSTs time for other science goals, where the most interesting signal is expected at low energies (e.g. observing distant active galaxies or binary systems [274, 275], see also [151] for a review).

Our results demonstrate that assuming an optimistic Einasto profile for the DM density distribution in the Milky Way, with 500 hours of the Galactic Center observations with CTA we can probe annihilation cross-section below its thermal value. This is true if the level of systematic $1 \%$ will be achieved by the CTA collaboration. Of course, the main uncertainty to this conclusion comes from the measurement of the Dark Matter mass around the Milky Way center. If these recent claims of $[259,260]$ are confirmed, this sensitivity will be significantly lower. In such a case it will be important to study in more details the relation between possible signals from the Galactic Center and dwarf spheroidal galaxies.

In Sections 3 and 4 we discussed the constraints on the properties of DM particles 
that are either model-independent or applicable to wide classes of models. In Section 5 we considered in detail an example of combinations of different constraints, coming both from particle physics on one side and from cosmology and astrophysics on the other side. We have considered a simple model that contains a neutral fermion playing the role of Dark Matter and a scalar that mixes with the Higgs boson and mediates the interaction of Dark Matter with the Standard Model. We concentrated on the case of light Dark Matter fermion, such that traditional direct detection approaches are difficult to apply as the recoil of nuclei becomes weak. We also assumed that the mediator is lighter than DM. In this case, this mediator decays visibly, i.e. into the Standard Model particles. At the same time, Dark Matter self-interaction mediated by such a light scalar may result in interesting astrophysical phenomenology (discussed in Section 3). We have shown that in this simple model, first of all, the searches for the invisible Higgs decay at the LHC constrain the same combination of coupling constants that is relevant for DM direct detection. For a part of the parameter space of the model, the sensitivity of invisible Higgs decay searches is comparable with the constraints from existing direct detection experiments. Secondly, the constraints from accelerator searches for light scalars, when combined with the astrophysical constraints on DM self-interaction (or with the requirement of the correct DM abundance assuming thermal production) appear to be very strong. The sensitivity of future intensity frontier experiments (such as e.g. SHiP), again combined with cosmological and astrophysical constraints, may allow to probe the part of the parameter space of the model that, in terms of direct detection experiments, reaches the irreducible neutrino background.

We have considered so far only the simplest Dark Matter model of the kind. In particular, we assumed a scalar mediator and thermally produced Dark Matter. It is important to perform the full exploration of the parameter space and consider a more general class of models elsewhere.

In this thesis, our main motivation originated from the Dark Matter problem. Of course, it is very important to apply the astro-particle approach, combining model-independent constraints of different nature, to more realistic models that can explain not only Dark Matter but also neutrino masses and matter-antimatter asymmetry of the Universe. This ambitious goal, however, can be achieved only for very specific models. Model-independent or widely applicable results remain therefore a necessary ingredient for identifying a successful complete extension of the Standard Model. 



\section{Chapter 7}

\section{Papers}






\section{Appendix A}

\section{Jeans equation}

The collisionless Boltzmann equation describes stellar dynamics [175],

$$
\frac{\partial f}{\partial t}+\boldsymbol{v} \cdot \nabla f-\nabla \Phi \cdot \frac{\partial f}{\partial \boldsymbol{v}}=0
$$

where $f$ is the distribution function, $\boldsymbol{v}$ is the DM velocity and $\Phi$ is the gravitational potential. Integrating it over all possible velocities one gets,

$$
\int \frac{\partial f}{\partial t} d^{3} \boldsymbol{v}+\int v_{i} \cdot \frac{\partial f}{\partial x_{i}} d^{3} \boldsymbol{v}-\frac{\partial \Phi}{\partial x_{i}} \int \frac{\partial f}{\partial v_{i}} d^{3} \boldsymbol{v}=0
$$

The last term is equal to zero because

$$
\int \frac{\partial f}{\partial v_{i}} d^{3} \boldsymbol{v}=\int d^{2} v_{i}^{\perp} \int_{-\infty}^{\infty} \frac{\partial f}{\partial v_{i}} d v_{i}=\int d^{2} v_{i}^{\perp}\left[f\left(v_{i}=\infty\right)-f\left(v_{i}=-\infty\right)\right]=0
$$

where $d^{2} v_{i}^{\perp}$ is 2D integration in the plane orthogonal to $v_{i}$. Let us introduce the density of stars, $\rho(\boldsymbol{x})$, and the mean stellar velocity, $\overline{\boldsymbol{v}}(\boldsymbol{x})$,

$$
\rho(\boldsymbol{x}, t)=\int f(\boldsymbol{x}, \boldsymbol{v}, t) d^{3} \boldsymbol{v} ; \quad \bar{v}_{i}=\frac{1}{\rho(\boldsymbol{x}, t)} \int f(\boldsymbol{x}, \boldsymbol{v}, t) v_{i} d^{3} \boldsymbol{v} .
$$

Plugging these definitions into (A.2) we get

$$
\frac{\partial \rho(\boldsymbol{x}, t)}{\partial t}+\frac{\partial\left(\rho(\boldsymbol{x}, t) \bar{v}_{i}\right)}{\partial x_{i}}=0
$$

Now, let us multiply the Boltzmann equation (A.1) by $v_{j}$ and integrate over velocities again,

$$
\int v_{j} \frac{\partial f}{\partial t} d^{3} \boldsymbol{v}+\int v_{i} v_{j} \cdot \frac{\partial f}{\partial x_{i}} d^{3} \boldsymbol{v}-\frac{\partial \Phi}{\partial x_{i}} \int v_{j} \frac{\partial f}{\partial v_{i}} d^{3} \boldsymbol{v}=0 .
$$


This equation can be rewritten in the following form,

$$
\frac{\partial\left(\rho(\boldsymbol{x}, t) \bar{v}_{j}\right)}{\partial t}+\frac{\partial\left(\rho(\boldsymbol{x}, t) \overline{v_{i} v_{j}}\right)}{\partial x_{i}}+\rho(\boldsymbol{x}, t) \frac{\partial \Phi}{\partial x_{j}}=0
$$

where $\overline{v_{i} v_{j}}$ is

$$
\overline{v_{i} v_{j}}=\frac{1}{\rho(\boldsymbol{x}, t)} \int v_{i} v_{j} f(\boldsymbol{x}, \boldsymbol{v}, t) d^{3} \boldsymbol{v} .
$$

Subtracting Eq. (A.7) from $\bar{v}_{j}$ times Eq. (A.5) we obtain,

$$
\rho(\boldsymbol{x}, t) \frac{\partial \bar{v}_{j}}{\partial t}-\bar{v}_{j} \frac{\partial\left(\rho(\boldsymbol{x}, t) \bar{v}_{i}\right)}{\partial x_{i}}+\frac{\partial\left(\rho(\boldsymbol{x}, t) \overline{v_{i} v_{j}}\right)}{\partial x_{i}}+\rho(\boldsymbol{x}, t) \frac{\partial \Phi}{\partial x_{j}}=0 .
$$

We consider a situation when a halo did not have a recent mergers and virialized for a long time. This means that time dependence for all halo parameters is weak, halo parameters change for the cosmological time. Neglecting time derivative and using $\bar{v}_{r}=\bar{v}_{\theta}=0$ we get

$$
\frac{d\left(\rho(\boldsymbol{x}, t) \sigma_{r}^{2}\right)}{d r}+\frac{\rho(\boldsymbol{x}, t)}{r}\left[2 \sigma_{r}^{2}-\left(\sigma_{\theta}^{2}+\sigma_{\phi}^{2}\right)\right]=-\rho(\boldsymbol{x}, t) \frac{d \Phi}{d r} .
$$

The anisotropy of the velocity dispersion is defined as

$$
\beta(r)=1-\frac{\sigma_{\theta}^{2}+\sigma_{\phi}^{2}}{2 \sigma_{r}^{2}} .
$$

Therefore, the Jeans equation can be rewriten as

$$
\frac{d\left(\rho(\boldsymbol{x}, t) \sigma_{r}^{2}\right)}{d r}+\frac{2 \beta(r) \rho(\boldsymbol{x}, t)}{r} \sigma_{r}^{2}=-\rho(\boldsymbol{x}, t) \frac{d \Phi}{d r} .
$$




\section{Appendix B}

\section{Reconstructing the inner SIDM density from NFW fit obtained at large distances}

In this Appendix we discuss a method to find a velocity dispersion for a given density and asymmetry profiles. The solution of this problem is needed to use the condition of the equal kinetic energy 3.9 without taking data on the velocity dispersion from simulations.

As we know, the density, the radial velocity dispersion, and velocity anisotropy profiles of collisionless CDM haloes are connected through the Jeans equation:

$$
\frac{d}{d r}\left(\sigma_{r}^{2}(r) \rho(r)\right)+\frac{2}{r} \beta(r) \sigma_{r}^{2}(r) \rho(r)=-\rho(r) \frac{G M(r)}{r^{2}}
$$

Therefore, one can find the velocity dispersion profile in CDM haloes using as an input the profiles for density and velocity anisotropy. To show this, we introduce the function $f(r)=\sigma_{r}^{2}(r) \rho(r)$ to write Eq. B.1:

$$
\frac{d f}{d r}+\frac{2}{r} \beta(r) f(r)=-\rho_{\mathrm{DM}}(r) \frac{G M_{\mathrm{DM}}(r)}{r^{2}},
$$

and we use the method of variation of constants to solve this equation. The solution of the homogeneous equation

$$
\frac{d f}{d r}+\frac{2}{r} \beta(r) f(r)=0
$$

is

$$
f(r)=C_{1} e^{-2 \int_{r_{0}}^{r} \frac{\beta(y)}{y} d y} .
$$

We substitute this solution in Eq. B.2 with $C_{1} \rightarrow C_{1}(r)$ and get

$$
\frac{d C_{1}}{d r} e^{-2 \int_{r_{0}}^{r} \frac{\beta(y)}{y} d y}=-\rho(r) \frac{G M(r)}{r^{2}} .
$$


The general solution for $C_{1}(r)$ in this equation is

$$
C_{1}(r)=C-\int_{r_{0}}^{r} e^{2 \int_{r_{0}}^{x} \frac{\beta(y)}{y} d y} \rho(x) \frac{G M(x)}{x^{2}} d x .
$$

Thus, the velocity dispersion profile is given by

$$
\sigma_{r}^{2}(r) \rho(r)=C e^{-2 \int_{r_{0}}^{r} \frac{\beta(y)}{y} d y}-\int_{r_{0}}^{r} e^{2 \int_{r}^{x} \frac{\beta(y)}{y} d y} \frac{G M(x)}{x^{2}} \rho(x) d x .
$$

The constant $C$ can be fixed with the values of the density and the velocity dispersion at a radius $r=r_{0}$ in Eq. B.7: $C=\sigma_{r}^{2}\left(r_{0}\right) \rho_{\mathrm{CDM}}\left(r_{0}\right)$. Thus, we finally have:

$$
\sigma_{r}^{2}(r) \rho(r)=\sigma_{r}^{2}\left(r_{0}\right) \rho\left(r_{0}\right) e^{-2 \int_{r_{0}}^{r} \frac{\beta(y)}{y} d y}-\int_{r_{0}}^{r} e^{2 \int_{r}^{x} \frac{\beta(y)}{y} d y} \frac{G M(x)}{x^{2}} \rho(x) d x .
$$

In principle, we have the problem that we do not know the value of $\sigma_{r}(r)$ at any finite radius $r_{0}$, but if we assume that $\sigma_{r}^{2}(r) \rho(r) \rightarrow 0$ as $r \rightarrow \infty$, which is reasonable for CDM haloes, then we can choose the boundary condition $\sigma_{r}^{2}\left(10 r_{s}\right) \rho\left(10 r_{s}\right)=0$. Therefore, we can use the NFW profile for $\rho(r)$ and the ansatz for $\beta(r)$ to estimate $\sigma_{v}$. An example of the resulting velocity dispersion profiles is shown in the left panel of Fig. 3.12. The quality of the match to the simulated data is comparable in all the cases to these examples and we can see that the fit is reasonable. 


\section{Appendix C}

\section{Parametric models for the data}

Astronomical observations are often presented for some models for density profiles, baryonic contributions, etc. We select objects where the density profile is reasonably well fit to both a cored profile and an NFW profile at large radii, and where the two resulting masses inside the best measured distance are consistent with each other.

The cored profiles that we take into account are:

- Burkert profile

$$
\rho_{\text {Burk }}(r)=\frac{\rho_{0}}{\left(1+r / r_{0}\right)\left(1+\left(r / r_{0}\right)^{2}\right)} .
$$

- Pseudoisothermal profile

$$
\rho_{\mathrm{ISO}}(r)=\frac{\rho_{0}}{\left(1+\left(r / r_{0}\right)^{2}\right)}
$$

- Modified pseudoisothermal profile

$$
\rho_{\mathrm{ISO} 2}(r)=\frac{\rho_{0}}{\left(1+\left(r / r_{0}\right)^{2}\right)^{3 / 2}} .
$$

- Cored NFW profile:

$$
\rho_{\mathrm{cNFW}}=\frac{b \rho_{s}}{\left(1+b r / r_{s}\right)\left(1+r / r_{s}\right)^{2}}
$$

The presence of baryons can significantly modify the properties of dark matter density profiles (see e.g. [100] for an SIDM example). Baryons also introduce uncertainties in measurements of DM distributions. We only select objects that are sufficiently DM dominated. In this case the poorly known mass-to-light ratios for stars, lack of reliable model of DM-baryons interaction and other uncertainties related to the baryons do not affect our analysis. Concretely, 
We demand that at the largest radius where the mass is measured the contribution of $\mathrm{DM}$ is at least 4 times larger than the mass of the baryons.

The following is a complete list of objects that we selected for our analysis, followed by a brief description of the characteristics of each object class:

- Dwarf Spheroidal galaxies, [185, 311] (NFW and Burkert profiles): Sculptor, Leo I, Carina, Draco, Ursa Minor, Fornax.

- Low Surface Brightness, [312] (NFW and ISO profiles): F563-V2, F563-1, F583-4

- GHASP, [313] (NFW and ISO2): UGC-3876, UGC-4256, UGC-4456, UGC-4499, UGC-10310.

- THINGS, [220] (NFW and ISO profiles): NGC-2903 (outer), NGC-3198 (2 comp),NGC2403 (1 comp), NGC-2403 (2 comp), NGC-2841, NGC-3621, DDO-154

- LITTLE THINGS, [314] (NFW and ISO profiles): DDO-52, DDO-101, WLM, Haro-29, DDO-87, DDO-126, DDO-216.

- SPARC, [221] (NFW and Burk): F568-V1, NGC-24, NGC-2683, NGC-3769, NGC3953, NGC-3992, NGC-4100, NGC-4183, UGC-2259, UGC-5721, UGC-7690, UGC8490, UGC-9992, UGC-12506.

- Clusters [177] (NFW and cNFW): MS2137, A963, A383, A611, A2537, A2667. 


\section{Bibliography}

[1] ATLAS Collaboration, G. Aad et al., Observation of a new particle in the search for the Standard Model Higgs boson with the ATLAS detector at the LHC, Phys. Lett. B716 (2012) 1-29, [arXiv: 1207 . 7214]. ii, 1, 5

[2] CMS Collaboration, S. Chatrchyan et al., Observation of a new boson at a mass of $125 \mathrm{GeV}$ with the CMS experiment at the LHC, Phys. Lett. B716 (2012) 30-61, [arXiv:1207.7235]. ii, 1, 5

[3] ATLAS Collaboration, T. A. collaboration, Combined measurements of Higgs boson production and decay using up to $80 \mathrm{fb}^{-1}$ of proton-proton collision data at $\sqrt{s}=13$ TeV collected with the ATLAS experiment, . 1

[4] F. Bezrukov and M. Shaposhnikov, Why should we care about the top quark Yukawa coupling?, J. Exp. Theor. Phys. 120 (2015) 335-343, [arXiv:1411.1923]. [Zh. Eksp. Teor. Fiz.147,389(2015)]. 1

[5] F. Reines and C. L. Cowan, The neutrino, Nature 178 (1956) 446-449. 2

[6] B. Pontecorvo, Neutrino Experiments and the Problem of Conservation of Leptonic Charge, Sov. Phys. JETP 26 (1968) 984-988. [Zh. Eksp. Teor. Fiz.53,1717(1967)]. 2

[7] V. N. Gribov and B. Pontecorvo, Neutrino astronomy and lepton charge, Phys. Lett. 28B (1969) 493. 2

[8] W. C. Haxton, R. G. Hamish Robertson, and A. M. Serenelli, Solar Neutrinos: Status and Prospects, Ann. Rev. Astron. Astrophys. 51 (2013) 21-61, [arXiv:1208.5723]. 2

[9] T. Kajita, Nobel Lecture: Discovery of atmospheric neutrino oscillations, Rev. Mod. Phys. 88 (2016), no. 3 030501. 2

[10] X. Qian and J.-C. Peng, Physics with Reactor Neutrinos, Rept. Prog. Phys. 82 (2019) 036201, [arXiv:1801.05386]. 2

[11] T. Lasserre and H. W. Sobel, Reactor neutrinos, Comptes Rendus Physique 6 (2005) 749-757, [nucl-ex/0601013]. 2

[12] M. C. Gonzalez-Garcia and Y. Nir, Neutrino masses and mixing: Evidence and implications, Rev. Mod. Phys. 75 (2003) 345-402, [hep-ph/0202058]. 2 
[13] S. M. Bilenky, Neutrino oscillations: brief history and present status, in Proceedings, 22nd International Baldin Seminar on High Energy Physics Problems, Relativistic Nuclear Physics and Quantum Chromodynamics, (ISHEPP 2014): Dubna, Russia, September 15-20, 2014, 2014. [arXiv: 1408.2864 ]. 2

[14] Particle Data Group Collaboration, M. Tanabashi et al., Review of Particle Physics, Phys. Rev. D98 (2018), no. 3 030001. 2, 6, 28, 101, 104

[15] KM3Net Collaboration, S. Adrian-Martinez et al., Letter of intent for KM3NeT 2.0, J. Phys. G43 (2016), no. 8 084001, [arXiv: 1601.07459 ]. 3

[16] C. S. Wu, E. Ambler, R. W. Hayward, D. D. Hoppes, and R. P. Hudson, Experimental Test of Parity Conservation in Beta Decay, Phys. Rev. 105 (1957) 1413-1414. 2

[17] A. Boyarsky, O. Ruchayskiy, and M. Shaposhnikov, The Role of sterile neutrinos in cosmology and astrophysics, Ann. Rev. Nucl. Part. Sci. 59 (2009) 191-214, [arXiv:0901.0011]. 3, 22

[18] L. Cardani, Neutrinoless Double Beta Decay Overview, [arXiv:1810.12828]. 3

[19] P. A. M. Dirac, The quantum theory of the electron, Proc. Roy. Soc. Lond. A117 (1928) 610-624. 4

[20] K. Blum, R. Sato, and E. Waxman, Cosmic-ray Antimatter, [arXiv:1709.06507]. 4, 26

[21] G. Steigman, When Clusters Collide: Constraints On Antimatter On The Largest Scales, JCAP 0810 (2008) 001, [arXiv: 0808 . 1122]. 4

[22] A. D. Sakharov, Violation of CP Invariance, $C$ asymmetry, and baryon asymmetry of the universe, Pisma Zh. Eksp. Teor. Fiz. 5 (1967) 32-35. [Usp. Fiz. Nauk161,no.5,61(1991)]. 5

[23] M. Shaposhnikov, Baryon Asymmetry of the Universe and Neutrinos, Progress of Theoretical Physics 122 (07, 2009) 185-203, [http://oup.prod.sis.lan/ptp/article-pdf/122/1/185/5392307/ $122-1-185 \cdot \mathrm{pdf}$

5

[24] Supernova Search Team Collaboration, A. G. Riess et al., Observational evidence from supernovae for an accelerating universe and a cosmological constant, Astron. J. 116 (1998) 1009-1038, [astro-ph/9805201]. 5

[25] CDF Collaboration, F. Abe et al., The CDF Detector: An Overview, Nucl. Instrum. Meth. A271 (1988) 387-403. 6 
[26] D0 Collaboration, S. Abachi et al., The D0 Detector, Nucl. Instrum. Meth. A338 (1994) 185-253. 6

[27] L. Evans and P. Bryant, LHC Machine, JINST 3 (2008) S08001. 6

[28] K. Bondarenko, A. Boyarsky, T. Bringmann, and A. Sokolenko, Constraining self-interacting dark matter with scaling laws of observed halo surface densities, [arXiv:1712.06602]. 6, 21, 33, 39, 48, 50, 52, 53, 55, 57, 58, 59, 60, 71

[29] A. Sokolenko, K. Bondarenko, T. Brinckmann, J. Zavala, M. Vogelsberger, T. Bringmann, and A. Boyarsky, Towards an improved model of self-interacting dark matter haloes, JCAP 1812 (2018), no. 12 038, [arXiv : 1806.11539 ]. 6, $21,33,37,38,39,40,41,42,43,44,45,46,47,48,49,53,71$

[30] CTA Collaboration, T. Bringmann et al., Pre-construction estimates of the CTA sensitivity to a dark matter signal from the Galactic centre, 2019. In progress. 6 , $29,72,85,89,90$

[31] I. Boiarska, K. Bondarenko, A. Boyarsky, V. Gorkavenko, M. Ovchynnikov, and A. Sokolenko, Phenomenology of GeV-scale scalar portal, [arXiv: 1904.10447 ] 6, 98, 99, 100, 101, 103

[32] F. Zwicky, Die Rotverschiebung von extragalaktischen Nebeln, Helv. Phys. Acta 6 (1933) 110-127. [Gen. Rel. Grav.41,207(2009)]. 7

[33] D. Massari, M. A. Breddels, A. Helmi, L. Posti, A. G. A. Brown, and E. Tolstoy, Three-dimensional motions in the Sculptor dwarf galaxy as a glimpse of a new era, Nature Astronomy 2 (Nov, 2018) 156-161, [arXiv:1711.08945]. 9

[34] D. Massari, M. A. Breddels, A. Helmi, L. Posti, A. G. A. Brown, and E. Tolstoy, Three-dimensional motions in the sculptor dwarf galaxy as a glimpse of a new era, Nature Astronomy 2 (2018), no. 2 156-161. 9

[35] J. T. Kleyna, M. I. Wilkinson, N. W. Evans, and G. Gilmore, Ursa Major: A Missing low-mass CDM halo?, Astrophys. J. 630 (2005) L141-L144, [astro-ph/0507154]. 9

[36] M. G. Walker, M. Mateo, E. W. Olszewski, J. Penarrubia, N. W. Evans, and G. Gilmore, A Universal Mass Profile for Dwarf Spheroidal Galaxies, Astrophys. J. 704 (2009) 1274-1287, [arXiv:0 906.0341 ]. [Erratum: Astrophys. J.710,886(2010)]. 9, 13, 34, 61

[37] S. Giodini, L. Lovisari, E. Pointecouteau, S. Ettori, T. H. Reiprich, and H. Hoekstra, Scaling relations for galaxy clusters: properties and evolution, Space Sci. Rev. 177 (2013) 247-282, [arXiv: 1305 . 3286]. 10

[38] J. Silk et al., Particle Dark Matter: Observations, Models and Searches. Cambridge Univ. Press, Cambridge, 2010. 10, 14, 21, 24, 25, 85 
[39] C. S. Frenk, A. E. Evrard, S. D. M. White, and F. J. Summers, Galaxy dynamics in clusters, The Astrophysical Journal 472 (dec, 1996) 460-484. 10

[40] H. Hoekstra, Weak gravitational lensing, Proc. Int. Sch. Phys. Fermi 186 (2014) 59-100, [arXiv:1312.5981]. 11

[41] Planck Collaboration, N. Aghanim et al., Planck 2018 results. VI. Cosmological parameters, [arXiv:1807.06209]. 11,12,14

[42] M. Vogelsberger, S. Genel, V. Springel, P. Torrey, D. Sijacki, D. Xu, G. Snyder, S. Bird, D. Nelson, and L. Hernquist, Properties of galaxies reproduced by a hydrodynamic simulation, Nature 509 (May, 2014) 177-182. 12

[43] M. Vogelsberger, S. Genel, V. Springel, P. Torrey, D. Sijacki, D. Xu, G. Snyder, D. Nelson, and L. Hernquist, Introducing the Illustris Project: simulating the coevolution of dark and visible matter in the Universe, MNRAS 444 (Oct., 2014) $1518-1547$.

[44] J. Schaye, R. A. Crain, R. G. Bower, M. Furlong, M. Schaller, T. Theuns, C. Dalla Vecchia, C. S. Frenk, I. G. McCarthy, J. C. Helly, A. Jenkins, Y. M.

Rosas-Guevara, S. D. M. White, M. Baes, C. M. Booth, P. Camps, J. F. Navarro, Y. Qu, A. Rahmati, T. Sawala, P. A. Thomas, and J. Trayford, The EAGLE project: simulating the evolution and assembly of galaxies and their environments, MNRAS 446 (Jan., 2015) 521-554.

[45] V. Springel, R. Pakmor, A. Pillepich, R. Weinberger, D. Nelson, L. Hernquist, M. Vogelsberger, S. Genel, P. Torrey, F. Marinacci, and J. Naiman, First results from the IllustrisTNG simulations: matter and galaxy clustering, MNRAS 475 (Mar., 2018) 676-698. 12

[46] S. Tremaine and J. E. Gunn, Dynamical Role of Light Neutral Leptons in Cosmology, Phys. Rev. Lett. 42 (1979) 407-410. [,66(1979)]. 13, 36

[47] J. Miralda-Escude, A test of the collisional dark matter hypothesis from cluster lensing, Astrophys. J. 564 (2002) 60, [astro-ph/ 0002050 ].

[48] D. Gorbunov, A. Khmelnitsky, and V. Rubakov, Is gravitino still a warm dark matter candidate?, JHEP 12 (2008) 055, [arXiv: 0805 . 2836].

[49] A. Boyarsky, O. Ruchayskiy, and D. Iakubovskyi, A Lower bound on the mass of Dark Matter particles, JCAP 0903 (2009) 005, [arXiv: 0808 . 3902]. 61

[50] V. Domcke and A. Urbano, Dwarf spheroidal galaxies as degenerate gas of free fermions, JCAP 1501 (2015), no. 01 002, [arXiv: 1409.3167 ]. 13

[51] B. G. Giraud and R. Peschanski, Profile of a Galactic Spherical Cloud of Self-Gravitating Fermions, [arXiv:1806.07283]. 13, 14 
[52] S. D. M. White, C. S. Frenk, and M. Davis, Clustering in a neutrino-dominated universe, ApJ 274 (Nov., 1983) L1-L5. 14

[53] T. D. Brandt, Constraints on MACHO Dark Matter from Compact Stellar Systems in Ultra-Faint Dwarf Galaxies, Astrophys. J. 824 (2016), no. 2 L31, [arXiv:1605.03665].15

[54] H. Niikura et al., Microlensing constraints on primordial black holes with the Subaru/HSC Andromeda observation, [arXiv: 1701.02151$] .15$

[55] S. Clesse and J. Garcia-Bellido, Seven Hints for Primordial Black Hole Dark Matter, Phys. Dark Univ. 22 (2018) 137-146, [arXiv:1711.10458]. 15

[56] S. Clesse and J. Garcia-Bellido, Massive Primordial Black Holes from Hybrid Inflation as Dark Matter and the seeds of Galaxies, Phys. Rev. D92 (2015), no. 2 023524, [arXiv:1501.07565]. 15

[57] B. Carr, Primordial black holes as dark matter and generators of cosmic structure, in Symposium on Illuminating Dark Matter Kruen, Germany, May 13-19, 2018, 2019. [arXiv:1901.07803]. 15

[58] T. Bringmann, P. F. Depta, V. Domcke, and K. Schmidt-Hoberg, Strong constraints on clustered primordial black holes as dark matter, [arXiv: 1808 .05910]. 15

[59] S. Clesse, J. García-Bellido, and S. Orani, Detecting the Stochastic Gravitational Wave Background from Primordial Black Hole Formation, [arXiv:1812.11011]. 15

[60] S. S. McGaugh, A tale of two paradigms: the mutual incommensurability of $\Lambda C D M$ and MOND, Can. J. Phys. 93 (2015), no. 2 250-259, [arXiv:1404.7525]. 15

[61] D. Clowe, A. Gonzalez, and M. Markevitch, Weak-Lensing Mass Reconstruction of the Interacting Cluster 1E 0657-558: Direct Evidence for the Existence of Dark Matter, ApJ 604 (Apr, 2004) 596-603, [astro-ph/ 0312273 ]. 15

[62] L. Roszkowski, Particle dark matter: A Theorist's perspective, Pramana 62 (2004) 389-401, [hep-ph/ 0404052 ]. 17

[63] A. V. Maccio, S. Paduroiu, D. Anderhalden, A. Schneider, and B. Moore, Cores in warm dark matter haloes: a Catch 22 problem, Mon. Not. Roy. Astron. Soc. 424 (2012) 1105-1112, [arXiv:1202.1282]. 17

[64] V. Springel, The Cosmological simulation code GADGET-2, Mon. Not. Roy. Astron. Soc. 364 (2005) 1105-1134, [astro-ph/0 505010 ]. 18 
[65] M. Vogelsberger, S. Genel, V. Springel, P. Torrey, D. Sijacki, D. Xu, G. F. Snyder, S. Bird, D. Nelson, and L. Hernquist, Properties of galaxies reproduced by a hydrodynamic simulation, Nature 509 (2014) 177-182, [arXiv: 1405.1418 ]. 18

[66] K. Kashiyama and M. Oguri, Detectability of Small-Scale Dark Matter Clumps with Pulsar Timing Arrays, [arXiv:1801.07847]. 18

[67] G. Despali, S. Vegetti, S. D. M. White, C. Giocoli, and F. C. van den Bosch, Modelling the line-of-sight contribution in substructure lensing, Mon. Not. Roy. Astron. Soc. 475 (2018), no. 4 5424-5442, [arXiv: 1710 . 05029]. 18, 19

[68] K. T. Inoue, R. Takahashi, T. Takahashi, and T. Ishiyama, Constraints on warm dark matter from weak lensing in anomalous quadruple lenses, Mon. Not. Roy. Astron. Soc. 448 (2015), no. 3 2704-2716, [arXiv: 1409.1326 ]. 18

[69] D. Gilman, S. Birrer, T. Treu, C. R. Keeton, and A. Nierenberg, Probing the nature of dark matter by forward modelling flux ratios in strong gravitational lenses, Mon. Not. Roy. Astron. Soc. 481 (2018), no. 1819-834, [arXiv: 1712.0494 5].

[70] S. Birrer, A. Amara, and A. Refregier, Lensing substructure quantification in RXJ1131-1231: A $2 \mathrm{keV}$ lower bound on dark matter thermal relic mass, JCAP 1705 (2017), no. 05 037, [arXiv: 1702.00009 ]. 18

[71] Euclid Theory Working Group Collaboration, L. Amendola et al., Cosmology and fundamental physics with the Euclid satellite, Living Rev. Rel. 16 (2013) 6, [arXiv:1206.1225]. 18

[72] L. Amendola et al., Cosmology and fundamental physics with the Euclid satellite, Living Rev. Rel. 21 (2018), no. 12, [arXiv: 1606.00180 ]. 18

[73] J. S. Bullock and M. Boylan-Kolchin, Small-Scale Challenges to the $\Lambda C D M$ Paradigm, Ann. Rev. Astron. Astrophys. 55 (2017) 343-387, [arXiv:1707.04256]. 18, 20

[74] B. Moore, S. Ghigna, F. Governato, G. Lake, T. R. Quinn, J. Stadel, and P. Tozzi, Dark matter substructure within galactic halos, Astrophys. J. 524 (1999) L19-L22, [astro-ph/9907411]. 18

[75] A. A. Klypin, A. V. Kravtsov, O. Valenzuela, and F. Prada, Where are the missing Galactic satellites?, Astrophys. J. 522 (1999) 82-92, [astro-ph/9901240].

[76] A. Fattahi, J. F. Navarro, T. Sawala, C. S. Frenk, L. V. Sales, K. Oman, M. Schaller, and J. Wang, The cold dark matter content of Galactic dwarf spheroidals: no cores, no failures, no problem, [arXiv:1607.06479]. 18 
[77] R. A. Flores and J. R. Primack, Observational and theoretical constraints on singular dark matter halos, Astrophys. J. 427 (1994) L1-4, [astro-ph/9402004]. 18

[78] B. Moore, Evidence against dissipation-less dark matter from observations of galaxy haloes, Nature 370 (Aug., 1994) 629-631. 18

[79] M. Boylan-Kolchin, J. S. Bullock, and M. Kaplinghat, Too big to fail? The puzzling darkness of massive Milky Way subhaloes, Mon. Not. Roy. Astron. Soc. 415 (July, 2011) L40-L44, [arXiv:1103.0007]. 19

[80] M. Boylan-Kolchin, J. S. Bullock, and M. Kaplinghat, The Milky Way's bright satellites as an apparent failure of LCDM, Mon. Not. Roy. Astron. Soc. 422 (2012) 1203-1218, [arXiv:1111.2048]. 19

[81] K. A. Oman et al., The unexpected diversity of dwarf galaxy rotation curves, Mon. Not. Roy. Astron. Soc. 452 (2015), no. 4 3650-3665, [arXiv: 1504.01437 ]. 19

[82] K. A. Oman, J. F. Navarro, L. V. Sales, A. Fattahi, C. S. Frenk, T. Sawala, M. Schaller, and S. D. M. White, Missing dark matter in dwarf galaxies?, Mon. Not. Roy. Astron. Soc. 460 (2016), no. 4 3610-3623, [arXiv:1601.01026]. 19

[83] S. Tulin and H.-B. Yu, Dark Matter Self-interactions and Small Scale Structure, [arXiv: 1705 . 02358]. 19, 20, 21, 35, 47, 50, 92, 95

[84] S. Mashchenko, J. Wadsley, and H. M. P. Couchman, Stellar Feedback in Dwarf Galaxy Formation, Science 319 (2008) 174, [arXiv: 0711 . 4803]. 19

[85] J. Wang, C. S. Frenk, J. F. Navarro, L. Gao, and T. Sawala, The missing massive satellites of the Milky Way, Mon. Not. Roy. Astron. Soc. 424 (Aug., 2012) 2715-2721, [arXiv:1203.4097].

[86] T. Sawala et al., The APOSTLE simulations: solutions to the Local Group's cosmic puzzles, Mon. Not. Roy. Astron. Soc. 457 (2016), no. 2 1931-1943, [arXiv:1511.01098]. 59, 64, 70

[87] Q. Guo, A. P. Cooper, C. Frenk, J. Helly, and W. A. Hellwing, The Milky Way system in $\Lambda$ cold dark matter cosmological simulations, Mon. Not. Roy. Astron. Soc. 454 (Nov., 2015) 550-559, [arXiv:1503.08508]. 19

[88] A. Garzilli, A. Boyarsky, and O. Ruchayskiy, Cutoff in the Lyman $\alpha$ forest power spectrum: warm IGM or warm dark matter?, Phys. Lett. B773 (2017) 258-264, [arXiv:1510.07006]. 19

[89] A. Garzilli, A. Magalich, T. Theuns, C. S. Frenk, C. Weniger, O. Ruchayskiy, and A. Boyarsky, The Lyman- $\alpha$ forest as a diagnostic of the nature of the dark matter, [arXiv:1809.06585]. 19 
[90] R. Li, C. S. Frenk, S. Cole, Q. Wang, and L. Gao, Projection effects in the strong lensing study of subhaloes, Mon. Not. Roy. Astron. Soc. 468 (2017), no. 2 1426-1432, [arXiv:1612.06227]. 19

[91] XENON Collaboration, E. Aprile et al., First Dark Matter Search Results from the XENON1T Experiment, Phys. Rev. Lett. 119 (2017), no. 18 181301, [arXiv:1705.06655]. 19

[92] PandaX-II Collaboration, X. Cui et al., Dark Matter Results From 54-Ton-Day Exposure of PandaX-II Experiment, Phys. Rev. Lett. 119 (2017), no. 18181302 , [arXiv:1708.06917]. 19

[93] D. N. Spergel and P. J. Steinhardt, Observational evidence for selfinteracting cold dark matter, Phys. Rev. Lett. 84 (2000) 3760-3763, [astro-ph/ 9909386 ]. 20

[94] A. Loeb and N. Weiner, Cores in Dwarf Galaxies from Dark Matter with a Yukawa Potential, Phys. Rev. Lett. 106 (2011) 171302, [arXiv:1011.6374]. 20

[95] M. Vogelsberger, J. Zavala, and A. Loeb, Subhaloes in Self-Interacting Galactic Dark Matter Haloes, Mon. Not. Roy. Astron. Soc. 423 (2012) 3740, [arXiv:1201.5892]. 20, 34, 59, 64, 70, 87

[96] A. H. G. Peter, M. Rocha, J. S. Bullock, and M. Kaplinghat, Cosmological Simulations with Self-Interacting Dark Matter II: Halo Shapes vs. Observations, Mon. Not. Roy. Astron. Soc. 430 (2013) 105, [arXiv: 1208 . 3026]. 20

[97] J. Zavala, M. Vogelsberger, and M. G. Walker, Constraining Self-Interacting Dark Matter with the Milky Way's dwarf spheroidals, Mon. Not. Roy. Astron. Soc. 431 (2013) L20-L24, [arXiv:1211.6426]. 20, 34

[98] L. G. van den Aarssen, T. Bringmann, and C. Pfrommer, Is dark matter with long-range interactions a solution to all small-scale problems of $\Lambda$ CDM cosmology?, Phys. Rev. Lett. 109 (2012) 231301, [arXiv:1205. 5809 ]. 20

[99] O. D. Elbert, J. S. Bullock, S. Garrison-Kimmel, M. Rocha, J. Oñorbe, and A. H. G. Peter, Core formation in dwarf haloes with self-interacting dark matter: no fine-tuning necessary, Mon. Not. Roy. Astron. Soc. 453 (2015), no. 1 29-37, [arXiv:1412.1477]. 57, 58

[100] A. Kamada, M. Kaplinghat, A. B. Pace, and H.-B. Yu, How the Self-Interacting Dark Matter Model Explains the Diverse Galactic Rotation Curves, Phys. Rev. Lett. 119 (2017), no. 11 111102, [arXiv: 1611 .02716]. 20, 50, 51, 229

[101] A. Robertson, R. Massey, V. Eke, S. Tulin, H.-B. Yu, Y. Bahé, D. J. Barnes, C. D. Vecchia, and S. T. Kay, The diverse density profiles of galaxy clusters with self-interacting dark matter plus baryons, [arXiv:1711.09096]. 20 
[102] X. Chu and B. Dasgupta, Dark Radiation Alleviates Problems with Dark Matter Halos, Phys. Rev. Lett. 113 (2014), no. 16 161301, [arXiv: 1404 . 6127]. 20

[103] M. Vogelsberger, J. Zavala, F.-Y. Cyr-Racine, C. Pfrommer, T. Bringmann, and K. Sigurdson, ETHOS - An Effective Theory of Structure Formation: Dark matter physics as a possible explanation of the small-scale CDM problems, Mon. Not. Roy. Astron. Soc. 460 (2016) 1399, [arXiv: 1512 . 0534 9]. 20

[104] T. Bringmann, J. Hasenkamp, and J. Kersten, Tight bonds between sterile neutrinos and dark matter, JCAP 1407 (2014) 042, [arXiv : 1312 . 494 7]. 20

[105] M. Kaplinghat, S. Tulin, and H.-B. Yu, Dark Matter Halos as Particle Colliders: Unified Solution to Small-Scale Structure Puzzles from Dwarfs to Clusters, Phys. Rev. Lett. 116 (2016), no. 4 041302, [arXiv: 1508 . 03339]. 20, 21, 34, 47, 50, 51

[106] M. Valli and H.-B. Yu, Dark matter self-interactions from the internal dynamics of dwarf spheroidals, [arXiv:1711.03502]. 20, 50, 51

[107] N. Yoshida, V. Springel, S. D. M. White, and G. Tormen, Weakly self-interacting dark matter and the structure of dark halos, Astrophys. J. 544 (2000) L87-L90, [astro-ph/0006134]. 20, 34

[108] O. Y. Gnedin and J. P. Ostriker, Limits on collisional dark matter from elliptical galaxies in clusters, Astrophys. J. 561 (2001) 61, [astro-ph/ 0010436 ].

[109] C. Firmani, E. D’Onghia, G. Chincarini, X. Hernandez, and V. Avila-Reese, Constraints on dark matter physics from dwarf galaxies through galaxy cluster haloes, Mon. Not. Roy. Astron. Soc. 321 (2001) 713, [a stro-ph/ 0005001 ].

[110] R. Dave, D. N. Spergel, P. J. Steinhardt, and B. D. Wandelt, Halo properties in cosmological simulations of selfinteracting cold dark matter, Astrophys. J. 547 (2001) 574-589, [astro-ph/0 006218 ]. 33

[111] P. Colin, V. Avila-Reese, O. Valenzuela, and C. Firmani, Structure and subhalo population of halos in a selfinteracting dark matter cosmology, Astrophys. J. 581 (2002) 777-793, [astro-ph/0205322].

[112] N. Arkani-Hamed, D. P. Finkbeiner, T. R. Slatyer, and N. Weiner, A Theory of Dark Matter, Phys. Rev. D79 (2009) 015014, [arXiv: 0810 . 0713].

[113] L. Ackerman, M. R. Buckley, S. M. Carroll, and M. Kamionkowski, Dark Matter and Dark Radiation, Phys. Rev. D79 (2009) 023519, [arXiv: 0810.5126 ]. [,277(2008)].

[114] J. L. Feng, M. Kaplinghat, H. Tu, and H.-B. Yu, Hidden Charged Dark Matter, JCAP 0907 (2009) 004, [arXiv: 0905 . 3039]. 
[115] M. R. Buckley and P. J. Fox, Dark Matter Self-Interactions and Light Force Carriers, Phys. Rev. D81 (2010) 083522, [arXiv: 0911 .3898].

[116] J. L. Feng, M. Kaplinghat, and H.-B. Yu, Halo Shape and Relic Density Exclusions of Sommerfeld-Enhanced Dark Matter Explanations of Cosmic Ray Excesses, Phys. Rev. Lett. 104 (2010) 151301, [arXiv:0911.0422].

[117] S. Tulin, H.-B. Yu, and K. M. Zurek, Oscillating Asymmetric Dark Matter, JCAP 1205 (2012) 013, [arXiv: 1202 . 0283].

[118] M. Rocha, A. H. G. Peter, J. S. Bullock, M. Kaplinghat, S. Garrison-Kimmel, J. Onorbe, and L. A. Moustakas, Cosmological Simulations with Self-Interacting Dark Matter I: Constant Density Cores and Substructure, Mon. Not. Roy. Astron. Soc. 430 (2013) 81-104, [arXiv: 1208 . 3025]. 34, 57, 58

[119] A. Robertson, R. Massey, V. Eke, and R. Bower, Self-Interacting Dark Matter Scattering Rates Through Cosmic Time, Mon. Not. Roy. Astron. Soc. 453 (2015), no. 3 2267-2276, [arXiv: 1505.02046 ].

[120] F.-Y. Cyr-Racine, K. Sigurdson, J. Zavala, T. Bringmann, M. Vogelsberger, and C. Pfrommer, ETHOS - an effective theory of structure formation: From dark particle physics to the matter distribution of the Universe, Phys. Rev. D93 (2016), no. 12 123527, [arXiv: 1512.0534 4].

[121] D. Harvey, A. Robertson, R. Massey, and J.-P. Kneib, Looking for dark matter trails in colliding galaxy clusters, [arXiv: 1610.05327$]$.

[122] S. Y. Kim, A. H. G. Peter, and D. Wittman, In the Wake of Dark Giants: New Signatures of Dark Matter Self Interactions in Equal Mass Mergers of Galaxy Clusters, Mon. Not. Roy. Astron. Soc. 469 (2017) 1414, [arXiv: 1608.08630 ]. 20

[123] T. Bringmann, J. Edsjö, P. Gondolo, P. Ullio, and L. Bergström, DarkSUSY 6 : An Advanced Tool to Compute Dark Matter Properties Numerically, JCAP 1807 (2018), no. 07 033, [arXiv: 1802 .03399]. 20, 95, 96

[124] T. Brinckmann, J. Zavala, D. Rapetti, S. H. Hansen, and M. Vogelsberger, The structure and assembly history of cluster-sized haloes in self-interacting dark matter, Mon. Not. Roy. Astron. Soc. 474 (2018), no. 1 746-759, [arXiv: 1705.00623 ]. 20, 38, 57, 58

[125] M. Vogelsberger, J. Zavala, K. Schutz, and T. R. Slatyer, Evaporating the Milky Way halo and its satellites with inelastic self-interacting dark matter, [arXiv:1805.03203].

[126] M. R. Buckley and A. H. G. Peter, Gravitational probes of dark matter physics, [arXiv:1712.06615]. 20 
[127] S. W. Randall, M. Markevitch, D. Clowe, A. H. Gonzalez, and M. Bradac, Constraints on the Self-Interaction Cross-Section of Dark Matter from Numerical Simulations of the Merging Galaxy Cluster 1E 0657-56, Astrophys. J. 679 (2008) 1173-1180, [arXiv:0704.0261]. 20

[128] D. Harvey, R. Massey, T. Kitching, A. Taylor, and E. Tittley, The non-gravitational interactions of dark matter in colliding galaxy clusters, Science 347 (2015) 1462-1465, [arXiv:1503.07675].

[129] A. Robertson, R. Massey, and V. Eke, What does the Bullet Cluster tell us about self-interacting dark matter?, Mon. Not. Roy. Astron. Soc. 465 (2017), no. 1 569-587, [arXiv:1605.04307].

[130] D. Wittman, N. Golovich, and W. A. Dawson, The Mismeasure of Mergers: Revised Limits on Self-interacting Dark Matter in Merging Galaxy Clusters, [arXiv: 1701.05877 ]. 20

[131] O. D. Elbert, J. S. Bullock, M. Kaplinghat, S. Garrison-Kimmel, A. S. Graus, and M. Rocha, A Testable Conspiracy: Simulating Baryonic Effects on Self-Interacting Dark Matter Halos, [arXiv: 1609 . 08626]. 21, 50, 51

[132] V. H. Robles, J. S. Bullock, O. D. Elbert, A. Fitts, A. González-Samaniego, M. Boylan-Kolchin, P. F. Hopkins, C.-A. Faucher-Giguère, D. Kereš, and C. C. Hayward, SIDM on FIRE: Hydrodynamical Self-Interacting Dark Matter simulations of low-mass dwarf galaxies, ArXiv e-prints (June, 2017) [arXiv:1706.07514]. 21

[133] L. E. Strigari, C. S. Frenk, and S. D. M. White, Dynamical constraints on the dark matter distribution of the Sculptor dwarf spheroidal from stellar proper motions, [arXiv:1801.07343]. 21

[134] M. Kaplinghat, R. E. Keeley, T. Linden, and H.-B. Yu, Tying Dark Matter to Baryons with Self-interactions, Phys. Rev. Lett. 113 (2014) 021302, [arXiv:1311.6524]. 21

[135] G. B. Gelmini, The Hunt for Dark Matter, in Proceedings, Theoretical Advanced Study Institute in Elementary Particle Physics: Journeys Through the Precision Frontier: Amplitudes for Colliders (TASI 2014): Boulder, Colorado, June 2-27, 2014, pp. 559-616, 2015. [arXiv:1502.01320]. 21

[136] D. Hooper, TASI Lectures on Indirect Searches For Dark Matter, [arXiv:1812.02029]. 21

[137] B. W. Lee and S. Weinberg, Cosmological Lower Bound on Heavy Neutrino Masses, Phys. Rev. Lett. 39 (1977) 165-168. [,183(1977)]. 21 
[138] A. Boyarsky, M. Drewes, T. Lasserre, S. Mertens, and O. Ruchayskiy, Sterile Neutrino Dark Matter, Prog. Part. Nucl. Phys. 104 (2019) 1-45, [arXiv:1807.07938]. 22

[139] K. N. Abazajian et al., Light Sterile Neutrinos: A White Paper, [arXiv: 1204.5379 ]. 22

[140] T. Asaka, S. Blanchet, and M. Shaposhnikov, The nuMSM, dark matter and neutrino masses, Phys. Lett. B631 (2005) 151-156, [hep-ph/ 0503065$] .23$

[141] T. Asaka and M. Shaposhnikov, The nuMSM, dark matter and baryon asymmetry of the universe, Phys. Lett. B620 (2005) 17-26, [hep-ph/ 0505013 ]. 23

[142] A. Boyarsky, A. Neronov, O. Ruchayskiy, and M. Shaposhnikov, The Masses of active neutrinos in the nuMSM from X-ray astronomy, JETP Lett. 83 (2006) 133-135, [hep-ph/0601098]. 23

[143] J. Jaeckel and A. Ringwald, The Low-Energy Frontier of Particle Physics, Ann. Rev. Nucl. Part. Sci. 60 (2010) 405-437, [arXiv : 1002 . 0329]. 22

[144] P. Sikivie, Axion Cosmology, Lect. Notes Phys. 741 (2008) 19-50, [astro-ph/0610440]. [,19(2006)]. 22

[145] R. D. Peccei and H. R. Quinn, CP Conservation in the Presence of Instantons, Phys. Rev. Lett. 38 (1977) 1440-1443. [,328(1977)]. 22

[146] L. Hui, J. P. Ostriker, S. Tremaine, and E. Witten, Ultralight scalars as cosmological dark matter, Phys. Rev. D95 (2017), no. 4 043541, [arXiv:1610.08297]. 22

[147] J. Preskill, M. B. Wise, and F. Wilczek, Cosmology of the Invisible Axion, Phys. Lett. B120 (1983) 127-132. [,URL(1982)]. 22

[148] X.-J. Bi, P.-F. Yin, and Q. Yuan, Status of Dark Matter Detection, Front. Phys.(Beijing) 8 (2013) 794-827, [arXiv: 1409.4590 ]. 23

[149] S.-C. Yoon, F. Iocco, and S. Akiyama, Evolution of the First Stars with Dark Matter Burning, Astrophys. J. 688 (2008) L1-L5, [arXiv: 0806.2662 ]. 23

[150] A. Garcon et al., Constraints on bosonic dark matter from ultralow-field nuclear magnetic resonance, [arXiv: 1902.04644$] .23$

[151] Cherenkov Telescope Array Consortium Collaboration, B. S. Acharya et al., Science with the Cherenkov Telescope Array, [arXiv: 1709.07997 ]. 24, 26, $72,78,79,80,83,88,91,110$

[152] P. Sikivie, The emerging case for axion dark matter, Phys. Lett. B695 (2011) 22-25, [arXiv:1003.2426]. 24 
[153] XENON Collaboration, E. Aprile et al., Dark Matter Search Results from a One Ton-Year Exposure of XENONIT, Phys. Rev. Lett. 121 (2018), no. 11111302 , [arXiv:1805.12562]. 24

[154] XENON100 Collaboration, E. Aprile et al., Dark Matter Results from 225 Live Days of XENON100 Data, Phys. Rev. Lett. 109 (2012) 181301, [arXiv:1207.5988]. 25

[155] LUX Collaboration, D. S. Akerib et al., First results from the LUX dark matter experiment at the Sanford Underground Research Facility, Phys. Rev. Lett. 112 (2014) 091303, [arXiv:1310.8214]. 25

[156] D. Yu. Akimov et al., WIMP-nucleon cross-section results from the second science run of ZEPLIN-III, Phys. Lett. B709 (2012) 14-20, [arXiv:1110 . 4769]. 25

[157] EDELWEISS Collaboration, E. Armengaud et al., Final results of the EDELWEISS-II WIMP search using a 4-kg array of cryogenic germanium detectors with interleaved electrodes, Phys. Lett. B702 (2011) 329-335, [arXiv:1103.4070]. 25

[158] G. Angloher et al., Results from $730 \mathrm{~kg}$ days of the CRESST-II Dark Matter Search, Eur. Phys. J. C72 (2012) 1971, [arXiv:1109.0702]. 25

[159] CRESST Collaboration, A. H. Abdelhameed et al., First results from the CRESST-III low-mass dark matter program, [arXiv: 1904 . 004 98]. 25, 106

[160] ADMX Collaboration, G. Rybka, Direct Detection Searches for Axion Dark Matter, Phys. Dark Univ. 4 (2014) 14-16. 26

[161] I. P. Stern, Axion Dark Matter Searches, AIP Conf. Proc. 1604 (2015), no. 1 456-461, [arXiv:1403.5332]. 26

[162] ADMX Collaboration, N. Du et al., A Search for Invisible Axion Dark Matter with the Axion Dark Matter Experiment, Phys. Rev. Lett. 120 (2018), no. 15 151301, [arXiv: 1804.05750 ]. 25

[163] T. Bringmann and C. Weniger, Gamma Ray Signals from Dark Matter: Concepts, Status and Prospects, Phys. Dark Univ. 1 (2012) 194-217, [arXiv:1208.5481]. 26, 27, 28

[164] H.E.S.S. Collaboration, L. Rinchiuso, Latest results on dark matter searches with H.E.S.S, in 7th Roma International Conference on Astroparticle Physic (RICAP18) Rome, Italy, September 4-7, 2018, 2019. [arXiv:1901.05299]. 28

[165] B. Anderson, S. Zimmer, J. Conrad, M. Gustafsson, M. Sánchez-Conde, and R. Caputo, Search for Gamma-Ray Lines towards Galaxy Clusters with the Fermi-LAT, JCAP 1602 (2016), no. 02 026, [arXiv: 1511.00014 ]. 28 
[166] L. Bergstrom and H. Snellman, Observable Monochromatic Photons From Cosmic Photino Annihilation, Phys. Rev. D37 (1988) 3737-3741. 28

[167] C. Arina, T. Bringmann, J. Silk, and M. Vollmann, Enhanced Line Signals from Annihilating Kaluza-Klein Dark Matter, Phys. Rev. D90 (2014), no. 8 083506, [arXiv:1409.0007]. 28

[168] H. Hu, Status of the EAS studies of cosmic rays with energy below $10 * * 16 \mathrm{eV}$, [arXiv:0911.3034]. 30

[169] Fermi-LAT Collaboration, M. Ackermann et al., The Fermi Galactic Center GeV Excess and Implications for Dark Matter, Astrophys. J. 840 (2017), no. 1 43, [arXiv:1704.03910]. 29

[170] C. Eckner et al., Millisecond pulsar origin of the Galactic center excess and extended gamma-ray emission from Andromeda - a closer look, Astrophys. J. 862 (2018), no. 1 79, [arXiv:1711.05127]. 29

[171] F. Aharonian, Very High Energy Cosmic Gamma Radiation: A Crucial Window on the Extreme Universe. EBSCO ebook academic collection. World Scientific, 2004. 31

[172] ATLAS Collaboration, G. Aad et al., Search for invisible decays of a Higgs boson using vector-boson fusion in pp collisions at $\sqrt{s}=8 \mathrm{TeV}$ with the ATLAS detector, JHEP 01 (2016) 172, [arXiv: 1508 .07869]. 32

[173] LDMX Collaboration, J. Mans, The LDMX Experiment, EPJ Web Conf. 142 (2017) 01020. 32

[174] NA62 Collaboration, E. Cortina Gil et al., The Beam and detector of the NA62 experiment at CERN, JINST 12 (2017), no. 05 P05025, [arXiv: 1703.08501 ]. 32

[175] J. Binney and S. Tremaine, Galactic dynamics. Princeton University Press, 1987. $33,44,225$

[176] J. Pollack, D. N. Spergel, and P. J. Steinhardt, Supermassive Black Holes from Ultra-Strongly Self-Interacting Dark Matter, Astrophys. J. 804 (2015), no. 2 131, [arXiv:1501.00017]. 33

[177] A. B. Newman, T. Treu, R. S. Ellis, and D. J. Sand, The Density Profiles of Massive, Relaxed Galaxy Clusters: II. Separating Luminous and Dark Matter in Cluster Cores, Astrophys. J. 765 (2013) 25, [arXiv: 1209 .1392]. 34, 63, 230

[178] B. Moore, S. Gelato, A. Jenkins, F. R. Pearce, and V. Quilis, Collisional versus collisionless dark matter, Astrophys. J. 535 (2000) L21-L24, [astro-ph/0002308]. 34 
[179] N. Yoshida, V. Springel, S. D. M. White, and G. Tormen, Collisional dark matter and the structure of dark halos, Astrophys. J. 535 (2000) L103, [astro-ph/0002362].

[180] M. Vogelsberger, J. Zavala, C. Simpson, and A. Jenkins, Dwarf galaxies in CDM and SIDM with baryons: observational probes of the nature of dark matter, Mon. Not. Roy. Astron. Soc. 444 (2014), no. 4 3684-3698, [arXiv: 1405.5216 ]. 34

[181] D. Lynden-Bell and R. Wood, The gravo-thermal catastrophe in isothermal spheres and the onset of red-giant structure for stellar systems, Mon. Not. Roy. Astron. Soc. 138 (1968) 495. 36

[182] J. Koda and P. R. Shapiro, Gravothermal collapse of isolated self-interacting dark matter haloes: N-body simulation versus the fluid model, Mon. Not. Roy. Astron. Soc. 415 (Aug., 2011) 1125-1137, [arXiv:1101.3097]. 36

[183] J. Pollack, D. N. Spergel, and P. J. Steinhardt, Supermassive Black Holes from Ultra-strongly Self-interacting Dark Matter, ApJ 804 (May, 2015) 131, [arXiv:1501.00017]. 36

[184] P. Creasey, O. Sameie, L. V. Sales, H.-B. Yu, M. Vogelsberger, and J. Zavala, Spreading out and staying sharp - creating diverse rotation curves via baryonic and self-interaction effects, Mon. Not. Roy. Astron. Soc. 468 (2017), no. 2 2283-2295, [arXiv:1612.03903]. 50, 51

[185] M. G. Walker. private communication. 50, 59, 230

[186] A. V. Maccio', A. A. Dutton, and F. C. v. d. Bosch, Concentration, Spin and Shape of Dark Matter Haloes as a Function of the Cosmological Model: WMAP1, WMAP3 and WMAP5 results, Mon. Not. Roy. Astron. Soc. 391 (2008) 1940-1954, [arXiv:0805.1926]. 51, 54

[187] F. Donato, G. Gentile, P. Salucci, C. F. Martins, M. I. Wilkinson, G. Gilmore, E. K. Grebel, A. Koch, and R. Wyse, A constant dark matter halo surface density in galaxies, Mon. Not. Roy. Astron. Soc. 397 (2009) 1169-1176, [arXiv:0904.4054]. 53

[188] G. Gentile, B. Famaey, H. Zhao, and P. Salucci, Universality of galactic surface densities within one dark halo scale-length, Nature 461 (2009) 627, [arXiv:0909.5203]. 53

[189] A. Boyarsky, O. Ruchayskiy, D. Iakubovskyi, A. V. Maccio', and D. Malyshev, New evidence for dark matter, [arXiv:0911.1774]. 53

[190] A. Boyarsky, A. Neronov, O. Ruchayskiy, and I. Tkachev, Universal properties of Dark Matter halos, Phys. Rev. Lett. 104 (2010) 191301, [arXiv:0911.3396]. 53 
[191] V. F. Cardone and C. Tortora, Dark matter scaling relations in intermediate $z$ haloes, Mon. Not. Roy. Astron. Soc. 409 (2010) 1570, [arXiv: 1007.3673 ]. 53

[192] N. R. Napolitano, A. J. Romanowsky, and C. Tortora, The central dark matter content of early-type galaxies: scaling relations and connections with star formation histories, Mon. Not. Roy. Astron. Soc. 405 (2010) 2351, [arXiv: 1003.1716$]$.

[193] A. Del Popolo, V. Cardone, and G. Belvedere, Surface Density of dark matter haloes on galactic and cluster scales, [arXiv: 1212.6797]. [Mon. Not. Roy. Astron. Soc.429,1080(2013)]. 53

[194] A. V. Maccio', A. A. Dutton, F. C. van den Bosch, B. Moore, D. Potter, and J. Stadel, Concentration, Spin and Shape of Dark Matter Haloes: Scatter and the Dependence on Mass and Environment, Mon. Not. Roy. Astron. Soc. 378 (2007) 55-71, [astro-ph/0608157]. 54

[195] A. F. Neto, L. Gao, P. Bett, S. Cole, J. F. Navarro, C. S. Frenk, S. D. M. White, V. Springel, and A. Jenkins, The statistics of lambda CDM Halo Concentrations, Mon. Not. Roy. Astron. Soc. 381 (2007) 1450-1462, [arXiv: 0706.2919 ].

[196] J. C. Munoz-Cuartas, A. V. Maccio, S. Gottlober, and A. A. Dutton, The Redshift Evolution of LCDM Halo Parameters: Concentration, Spin, and Shape, Mon. Not. Roy. Astron. Soc. 411 (2011) 584, [arXiv: 1007.0438$].$

[197] F. Prada, A. A. Klypin, A. J. Cuesta, J. E. Betancort-Rijo, and J. Primack, Halo concentrations in the standard $\Lambda$ cold dark matter cosmology, Mon. Not. Roy. Astron. Soc. 423 (July, 2012) 3018-3030, [arXiv: 1104 . 5130].

[198] A. Klypin, G. Yepes, S. Gottlober, F. Prada, and S. Hess, MultiDark simulations: the story of dark matter halo concentrations and density profiles, Mon. Not. Roy. Astron. Soc. 457 (2016), no. 4 4340-4359, [arXiv: 1411 . 4001 ].

[199] S. V. Pilipenko, M. A. Sánchez-Conde, F. Prada, and G. Yepes, Pushing down the low-mass halo concentration frontier with the Lomonosov cosmological simulations, Mon. Not. Roy. Astron. Soc. 472 (2017) 4918, [arXiv:1703.06012]. 54

[200] J. M. Comerford and P. Natarajan, The Observed Concentration-Mass Relation for Galaxy Clusters, Mon. Not. Roy. Astron. Soc. 379 (2007) 190-200, [astro-ph/0703126]. 54

[201] M. Sereno and G. Covone, The mass-concentration relation in massive galaxy clusters at redshift 1, Mon. Not. Roy. Astron. Soc. 434 (2013) 878, [arXiv:1306.6096].

[202] J. Merten et al., CLASH: The Concentration-Mass Relation of Galaxy Clusters, Astrophys. J. 806 (2015), no. 1 4, [arXiv: 1404.1376 ]. 
[203] W. Du, Z. Fan, H. Shan, G.-B. Zhao, G. Covone, L. Fu, and J.-P. Kneib, Mass-concentration relation of clusters of galaxies from CFHTLenS, Astrophys. J. 814 (2015), no. 2 120, [arXiv:1510.08193].

[204] A. M. Groener, D. M. Goldberg, and M. Sereno, The galaxy cluster concentration-mass scaling relation, Mon. Not. Roy. Astron. Soc. 455 (2016), no. 1 892-919, [arXiv:1510.01961].

[205] S. Amodeo, S. Ettori, R. Capasso, and M. Sereno, The relation between mass and concentration in X-ray galaxy clusters at high redshift, Astron. Astrophys. $\mathbf{5 9 0}$ (2016) A126, [arXiv: 1604.02163$].$

[206] N. Cibirka et al., CODEX weak lensing: concentration of galaxy clusters at $z \sim 0.5$, Mon. Not. Roy. Astron. Soc. 468 (2017), no. 1 1092-1116, [arXiv:1612.06871].

[207] K. Umetsu, A. Zitrin, D. Gruen, J. Merten, M. Donahue, and M. Postman, CLASH: Joint Analysis of Strong-Lensing, Weak-Lensing Shear and Magnification Data for 20 Galaxy Clusters, Astrophys. J. 821 (2016), no. 2 116, [arXiv: 1507.04385 ].

[208] M. Lieu, W. M. Farr, M. Betancourt, G. P. Smith, M. Sereno, and I. G. McCarthy, Hierarchical inference of the relationship between Concentration and Mass in Galaxy Groups and Clusters, Mon. Not. Roy. Astron. Soc. 468 (2017), no. 4 4872-4886, [arXiv:1701.00478]. 54

[209] W. A. Rolke, A. M. Lopez, and J. Conrad, Limits and confidence intervals in the presence of nuisance parameters, Nucl. Instrum. Meth. A551 (2005) 493-503, [physics/0403059]. 56

[210] A. Fitts et al., Dwarf Galaxies in CDM, WDM, and SIDM: Disentangling Baryons and Dark Matter Physics, [arXiv: 1811.11791]. 59, 64, 70

[211] A. Fattahi, J. F. Navarro, T. Sawala, C. S. Frenk, K. A. Oman, R. A. Crain, M. Furlong, M. Schaller, J. Schaye, T. Theuns, and A. Jenkins, The APOSTLE project: Local Group kinematic mass constraints and simulation candidate selection, MNRAS 457 (Mar, 2016) 844-856, [arXiv: 1507 .03643]. 59, 64

[212] V. Springel, J. Wang, M. Vogelsberger, A. Ludlow, A. Jenkins, A. Helmi, J. F. Navarro, C. S. Frenk, and S. D. M. White, The Aquarius Project: the subhalos of galactic halos, Mon. Not. Roy. Astron. Soc. 391 (2008) 1685-1711, [arXiv: 0809 .0898]. 59, 64, 65, 70, 87

[213] I. G. McCarthy, J. Schaye, S. Bird, and A. M. C. Le Brun, The BAHAMAS project: Calibrated hydrodynamical simulations for large-scale structure cosmology, Mon. Not. Roy. Astron. Soc. 465 (2017), no. 3 2936-2965, [arXiv: 1603 . 02702]. $59,65,70$ 
[214] A. Geringer-Sameth, S. M. Koushiappas, and M. Walker, Dwarf galaxy annihilation and decay emission profiles for dark matter experiments, Astrophys. J. 801 (2015), no. 2 74, [arXiv: 1408.0002 ]. 59

[215] M. G. Walker and J. Penarrubia, A Method for Measuring (Slopes of) the Mass Profiles of Dwarf Spheroidal Galaxies, Astrophys. J. 742 (2011) 20, [arXiv:1108.2404]. 60

[216] J. Wolf, G. D. Martinez, J. S. Bullock, M. Kaplinghat, M. Geha, R. R. Munoz, J. D. Simon, and F. F. Avedo, Accurate Masses for Dispersion-supported Galaxies, Mon. Not. Roy. Astron. Soc. 406 (2010) 1220, [arXiv: 0908 . 2995].

[217] N. C. Amorisco and N. W. Evans, Dark Matter Cores and Cusps: The Case of Multiple Stellar Populations in Dwarf Spheroidals, Mon. Not. Roy. Astron. Soc. 419 (2012) 184-196, [arXiv:1106.1062]. 60

[218] SDSS Collaboration, D. G. York et al., The Sloan Digital Sky Survey: Technical Summary, Astron. J. 120 (2000) 1579-1587, [astro-ph/ 0006396 ]. 61

[219] DES Collaboration, T. M. C. Abbott et al., Dark Energy Survey year 1 results: Cosmological constraints from galaxy clustering and weak lensing, Phys. Rev. $\mathbf{D 9 8}$ (2018), no. 4 043526, [arXiv:1708.01530]. 61

[220] W. J. G. de Blok, F. Walter, E. Brinks, C. Trachternach, S.-H. Oh, and R. C. Kennicutt, Jr., High-Resolution Rotation Curves and Galaxy Mass Models from THINGS, Astron. J. 136 (2008) 2648-2719, [arXiv: 0810 . 2100]. 61, 230

[221] F. Lelli, S. S. McGaugh, and J. M. Schombert, SPARC: Mass Models for 175 Disk Galaxies with Spitzer Photometry and Accurate Rotation Curves, arXiv (2016) [arXiv:1606.09251]. 62, 230

[222] J. Schombert, S. McGaugh, and F. Lelli, The mass-to-light ratios and the star formation histories of disc galaxies, MNRAS 483 (Feb., 2019) 1496-1512, [arXiv:1811.10579]. 62

[223] M. Schaller, C. S. Frenk, R. G. Bower, T. Theuns, J. Trayford, R. A. Crain, M. Furlong, J. Schaye, C. D. Vecchia, and I. G. McCarthy, The effect of baryons on the inner density profiles of rich clusters, Mon. Not. Roy. Astron. Soc. 452 (2015), no. $1343-355$, [arXiv:1409.8297]. 63

[224] H. Hoekstra, A. Mahdavi, A. Babul, and C. Bildfell, The Canadian Cluster Comparison Project: weak lensing masses and SZ scaling relations, Mon. Not. Roy. Astron. Soc. 427 (2012) 1298, [arXiv: 1208 . 0606 ]. 63

[225] J. Wang, J. F. Navarro, C. S. Frenk, S. D. M. White, V. Springel, A. Jenkins, A. Helmi, A. Ludlow, and M. Vogelsberger, Assembly History and Structure of Galactic Cold Dark Matter Halos, Mon. Not. Roy. Astron. Soc. 413 (2011) 1373, [arXiv: 1008.5114 ]. 65 
[226] A. Robertson, D. Harvey, R. Massey, V. Eke, I. G. McCarthy, M. Jauzac, B. Li, and J. Schaye, Observable tests of self-interacting dark matter in galaxy clusters: cosmological simulations with SIDM and baryons, [arXiv: 1810.05649 ]. 65

[227] Eagle, "The Eagle project." http://icc.dur.ac.uk/Eagle/publications.php, 2019. 71

[228] K. Bernlohr, Simulation of Imaging Atmospheric Cherenkov Telescopes with CORSIKA and sim-telarray, Astropart. Phys. 30 (2008) 149-158, [arXiv:0808.2253]. 72

[229] Y. Becherini, A. Djannati-Atai, V. Marandon, M. Punch, and S. Pita, A new analysis strategy for detection of faint gamma-ray sources with Imaging Atmospheric Cherenkov Telescopes, Astropart. Phys. 34 (2011) 858-870, [arXiv:1104.5359]. 72

[230] G. Di Sciascio, Ground-based Gamma-Ray Astronomy: an Introduction, in 18th International Baikal Summer School on Physics of Elementary Particles and Astrophysics: Exploring the Universe through multiple messengers (ISAPP-Baikal 2018) Bolshie Koty, Lake Baikal, Russia, July 12-21, 2018, 2019. [arXiv:1904.06218]. 72

[231] P. A. Cherenkov, Visible luminescence of pure liquids under the influence of gamma-radiation, Dokl. Akad. Nauk SSSR 2 (1934), no. 8 451-454. [Usp. Fiz. Nauk93,no.2,385(1967)]. 73

[232] I. M. Frank and I. E. Tamm, Coherent visible radiation of fast electrons passing through matter, Compt. Rend. Acad. Sci. URSS 14 (1937), no. 3 109-114. [Usp. Fiz. Nauk93,no.2,388(1967)]. 73, 75

[233] P. M. S. Blackett, A possible contribution to the night sky from the Cerenkov radiation emitted by cosmic rays, in The Emission Spectra of the Night Sky and Aurorae, p. 34, 1948. 75

[234] J. V. Jelley and N. A. Porter, Cerenkov Radiation from the Night Sky, QJRAS 4 (Mar., 1963) 275. 75

[235] R. M. Wagner, Measurement of Very High Energy Gamma-Ray Emission from Four Blazars Using the MAGIC Telescope and a Comparative Blazar Study. $\mathrm{PhD}$ thesis, Munich, Tech. U., 2006. 75

[236] Felix Aharonian, "Very High Energy Gamma Ray Domain.” https : //www.isdc.unige.ch/sf2010/lectures/FA_Lecture_3.pdf, 2010. [40th Saas-Fee Course: Astrophysics at Very High Energies]. 76

[237] J. Espadanal and P. Gonçalves, 3D simulation for Cherenkov emissions in Extensive Air Showers, [arXiv:1702.03276]. 77 
[238] C. Balázs, J. Conrad, B. Farmer, T. Jacques, T. Li, M. Meyer, F. S. Queiroz, and M. A. Sánchez-Conde, Sensitivity of the Cherenkov Telescope Array to the detection of a dark matter signal in comparison to direct detection and collider experiments, Phys. Rev. D96 (2017), no. 8 083002, [arXiv: 1706.01505 ]. 79, 82

[239] CTA Collaboration, J. Carr et al., Prospects for Indirect Dark Matter Searches with the Cherenkov Telescope Array (CTA), PoS ICRC2015 (2016) 1203, [arXiv:1508.06128]. [34,1203(2015)].

[240] CTA Consortium Collaboration, A. Morselli, The Dark Matter Programme of the Cherenkov Telescope Array, PoS ICRC2017 (2018) 921, [arXiv: 1709.01483$]$. [35,921(2017)].

[241] CTA Consortium Collaboration, M. Doro et al., Dark Matter and Fundamental Physics with the Cherenkov Telescope Array, Astropart. Phys. 43 (2013) 189-214, [arXiv:1208.5356].

[242] H. Silverwood, C. Weniger, P. Scott, and G. Bertone, A realistic assessment of the CTA sensitivity to dark matter annihilation, JCAP 1503 (2015), no. 03 055, [arXiv:1408.4131].

[243] M. Pierre, J. M. Siegal-Gaskins, and P. Scott, Sensitivity of CTA to dark matter signals from the Galactic Center, JCAP 1406 (2014) 024, [arXiv: 1401 . 7330]. [Erratum: JCAP1410,E01(2014)].

[244] V. Lefranc, E. Moulin, P. Panci, and J. Silk, Prospects for Annihilating Dark Matter in the inner Galactic halo by the Cherenkov Telescope Array, Phys. Rev. D91 (2015), no. 12 122003, [arXiv: 1502.05064 ]. 79

[245] M. Fairbairn, A. Nilsson, J. Ellis, J. Hinton, and R. White, The CTA Sensitivity to Lorentz-Violating Effects on the Gamma-Ray Horizon, JCAP 1406 (2014) 005, [arXiv: 1401.8178 ]. 79

[246] CTA Collaboration, M. K. Daniel, D. Emmanoulopoulos, M. Fairbairn, and N. Otte, Prospects on Testing Lorentz Invariance Violation with the Cherenkov Telescope Array, PoS ICRC2015 (2016) 708, [arXiv: 1508 . 06622].

[247] G. Rubtsov, P. Satunin, and S. Sibiryakov, Constraints on violation of Lorentz. invariance from atmospheric showers initiated by multi-TeV photons, JCAP 1705 (2017) 049, [arXiv:1611.10125]. 79

[248] CTA Consortium Collaboration, D. Mazin, M. Raue, B. Behera, S. Inoue, Y. Inoue, T. Nakamori, and T. Totani, Potential of EBL and cosmology studies with the Cherenkov Telescope Array, Astropart. Phys. 43 (2013) 241-251, [arXiv:1303.7124]. 79 
[249] CTA Consortium Collaboration, F. Gaté, R. Alves Batista, J. Biteau, J. Lefaucheur, S. Mangano, M. Meyer, Q. Piel, S. Pita, D. Sanchez, and I. Vovk, Studying cosmological $\gamma$-ray propagation with the Cherenkov Telescope Array, PoS ICRC2017 (2018) 623, [arXiv: 1709 . 04185 ]. [35,623(2017)]. 79

[250] M. Meyer, J. Conrad, and H. Dickinson, Sensitivity of the Cherenkov Telescope Array to the Detection of Intergalactic Magnetic Fields, Astrophys. J. 827 (2016), no. 2 147, [arXiv:1603.03431]. 79

[251] M. Fernández Alonso, A. D. Supanitsky, and A. C. Rovero, Probing the IGMF with the Next Generation of Cherenkov Telescopes, Astrophys. J. 869 (2018), no. 1 43, [arXiv:1810.08576]. 79

[252] M. Meyer and J. Conrad, Sensitivity of the Cherenkov Telescope Array to the detection of axion-like particles at high gamma-ray opacities, JCAP 1412 (2014), no. 12 016, [arXiv:1410.1556]. 79

[253] D. Malyshev, A. Neronov, D. Semikoz, A. Santangelo, and J. Jochum, Improved limit on axion-like particles from $\gamma$-ray data on Perseus cluster, [arXiv:1805.04388].

[254] H. Vogel, R. Laha, and M. Meyer, Diffuse axion-like particle searches, [arXiv:1712.01839]. 79

[255] J. Knödlseder et al., GammaLib and ctools: A software framework for the analysis of astronomical gamma-ray data, Astron. Astrophys. 593 (2016) A1, [arXiv: 1606.00393 ]. 80

[256] Fermi-LAT Collaboration, W. B. Atwood et al., The Large Area Telescope on the Fermi Gamma-ray Space Telescope Mission, Astrophys. J. 697 (2009) 1071-1102, [arXiv:0902.1089]. 80

[257] EGRET Collaboration, R. C. Hartman et al., The Third EGRET catalog of high-energy gamma-ray sources, Astrophys. J. Suppl. 123 (1999) 79. 80

[258] T. D. P. Edwards and C. Weniger, swordfish: Efficient Forecasting of New Physics Searches without Monte Carlo, [arXiv:1712.05401]. 85

[259] C. Wegg, O. Gerhard, and M. Bieth, The Gravitational Force Field of the Galaxy Measured From the Kinematics of RR Lyrae in Gaia, [arXiv:1806.09635]. $86,91,110$

[260] M. Portail, O. Gerhard, C. Wegg, and M. Ness, Dynamical modelling of the galactic bulge and bar: the Milky Way's pattern speed, stellar and dark matter mass distribution, Mon. Not. Roy. Astron. Soc. 465 (2017), no. 2 1621-1644, [arXiv:1608.07954]. 86, 91, 110 
[261] F. Iocco and M. Benito, An estimate of the DM profile in the Galactic bulge region, Physics of the Dark Universe 15 (Mar, 2017) 90-95, [arXiv: 1611.09861 ]. 86,87

[262] Gaia Collaboration, A. G. A. Brown et al., Gaia Data Release 2, Astron. Astrophys. 616 (2018) A1, [arXiv:1804.09365]. 86

[263] R. M. Rich, D. B. Reitzel, C. D. Howard, and H. Zhao, The Bulge Radial Velocity Assay (BRAVA): Techniques and a Rotation Curve, Astrophys. J. 658 (2007) L29-L32, [astro-ph/ 0611403 ]. 86

[264] A. Kunder et al., The Bulge Radial Velocity Assay (BRAVA): II. Complete Sample and Data Release, Astron. J. 143 (2012) 57, [arXiv:1112.1955]. 86

[265] N. J. Rattenbury, S. Mao, T. Sumi, and M. C. Smith, Modelling the Galactic bar using OGLE-II Red Clump Giant Stars, Mon. Not. Roy. Astron. Soc. 378 (2007) 1064-1078, [arXiv:0704.1614]. 86

[266] K. Freeman et al., ARGOS II: The Galactic Bulge Survey, Mon. Not. Roy. Astron. Soc. 428 (2013) 3660, [arXiv: 1212.1541 ]. 86

[267] M. Ness, K. Freeman, E. Athanassoula, E. W. de Boer, J. B. Hawthorn, M. Asplund, G. F. Lewis, D. Yong, R. R. Lane, and L. L. Kiss, ARGOS III: Stellar Populations in the Galactic Bulge of the Milky Way, Mon. Not. Roy. Astron. Soc. 430 (2013) 836, [arXiv:1212.1540]. 86

[268] M. Portail, O. Gerhard, and C. Wegg, The structure of the Milky Way's bar outside the bulge, Monthly Notices of the Royal Astronomical Society $450(05,2015)$ 4050-4069, [http://oup.prod.sis.lan/mnras/article-pdf/450/4/4050/ $5766721 /$ stv745.pdf 86

[269] Justin Read, "The Milky Way in the Gaia Era." https://www.physik.uzh.ch/events/sf2019/lectures.html, 2019. [Lectures at winter school ]. 87

[270] J. Green et al., Wide-Field InfraRed Survey Telescope (WFIRST) Final Report, arXiv e-prints (Aug, 2012) arXiv:1208.4012, [arXiv:1208.4012]. 86

[271] J. I. Read, M. G. Walker, and P. Steger, Dark matter heats up in dwarf galaxies, Mon. Not. Roy. Astron. Soc. 484 (2019), no. 1 1401-1420, [arXiv:1808.06634]. 87

[272] G. Cowan, K. Cranmer, E. Gross, and O. Vitells, Asymptotic formulae for likelihood-based tests of new physics, Eur. Phys. J. C71 (2011) 1554, [arXiv: 1007.1727 ]. [Erratum: Eur. Phys. J.C73,2501(2013)]. 89 
[273] D. Gaggero, D. Grasso, A. Marinelli, M. Taoso, and A. Urbano, Diffuse cosmic rays shining in the Galactic center: A novel interpretation of H.E.S.S. and Fermi-LAT $\gamma$-ray data, Phys. Rev. Lett. 119 (2017), no. 3 031101, [arXiv:1702.01124]. 89

[274] CTA Consortium Collaboration, A. Zech, AGN Physics with the Cherenkov Telescope Array, in Fermi and Jansky: Our Evolving Understanding of AGN Harbourtowne Conference Center, St Michaels, MD, USA, November 10-12, 2011, 2012. [arXiv:1205.1459]. 91, 110

[275] CTA Consortium Collaboration, J. M. Paredes et al., Binaries with the eyes of CTA, Astropart. Phys. 43 (2013) 301-316, [arXiv: 1210 . 3215]. 91, 110

[276] T. Bringmann and M. Pospelov, Novel direct detection constraints on light dark matter, [arXiv:1810.10543]. 92, 106, 107

[277] J. Alimena et al., Searching for long-lived particles beyond the Standard Model at the Large Hadron Collider, [arXiv: 1903.04497 ]. 92

[278] ATLAS Collaboration, M. Aaboud et al., Search for long-lived, massive particles in events with displaced vertices and missing transverse momentum in $\sqrt{s}=13$ TeV pp collisions with the ATLAS detector, Phys. Rev. D97 (2018), no. 5 052012, [arXiv:1710.04901]. 92

[279] G. Cottin, J. C. Helo, and M. Hirsch, Displaced vertices as probes of sterile neutrino mixing at the LHC, Phys. Rev. D98 (2018), no. 3 035012, [arXiv:1806.05191].

[280] S. Antusch, E. Cazzato, and O. Fischer, Sterile neutrino searches via displaced vertices at LHCb, Phys. Lett. B774 (2017) 114-118, [arXiv: 1706.05990$] .92$

[281] W. Bonivento et al., Proposal to Search for Heavy Neutral Leptons at the SPS, [arXiv:1310.1762]. 92

[282] SHiP Collaboration, M. Anelli et al., A facility to Search for Hidden Particles (SHiP) at the CERN SPS, [arXiv:1504.04956].

[283] SHiP Collaboration, C. Ahdida et al., The experimental facility for the Search for Hidden Particles at the CERN SPS, JINST (2019) [arXiv: 1810 . 06880]. 92, 102

[284] D. Curtin et al., Long-Lived Particles at the Energy Frontier: The MATHUSLA Physics Case, [arXiv:1806.07396]. 92, 102

[285] G. Krnjaic, Probing Light Thermal Dark-Matter With a Higgs Portal Mediator, Phys. Rev. D94 (2016), no. 7 073009, [arXiv:1512.04119]. 93, 105 
[286] S. Tulin, H.-B. Yu, and K. M. Zurek, Beyond Collisionless Dark Matter: Particle Physics Dynamics for Dark Matter Halo Structure, Phys. Rev. D87 (2013), no. 11 115007, [arXiv:1302.3898]. 95

[287] J. D. Clarke, R. Foot, and R. R. Volkas, Phenomenology of a very light scalar (100 $\left.\mathrm{MeV} ; m_{h} ; 10 \mathrm{GeV}\right)$ mixing with the SM Higgs, JHEP 02 (2014) 123, [arXiv: 1310.8042 ]. 98, 99, 103

[288] KTEV Collaboration, A. Alavi-Harati et al., Search for the Decay $K_{L} \rightarrow \pi^{0} \mu^{+} \mu^{-}$, Phys. Rev. Lett. 84 (2000) 5279-5282, [hep-ex/ 000100 6]. 102, 103

[289] LHCb Collaboration, R. Aaij et al., Search for long-lived scalar particles in $B^{+} \rightarrow K^{+} \chi\left(\mu^{+} \mu^{-}\right)$decays, Phys. Rev. D95 (2017), no. 7071101 , [arXiv:1612.07818]. 98, 103

[290] DUNE Collaboration, R. Acciarri et al., Long-Baseline Neutrino Facility (LBNF) and Deep Underground Neutrino Experiment (DUNE), [arXiv:1512.06148]. 99

[291] K. Bondarenko, A. Boyarsky, M. Ovchynnikov, and O. Ruchayskiy, Sensitivity of the intensity frontier experiments for neutrino and scalar portals: analytic estimates, [arXiv:1902.06240]. 99, 103

[292] F. Bezrukov, D. Gorbunov, and I. Timiryasov, Uncertainties of hadronic scalar decay calculations, [arXiv:1812.08088]. 99

[293] M. W. Winkler, Decay and Detection of a Light Scalar Boson Mixing with the Higgs, [arXiv:1809.01876]. 99

[294] A. Monin, A. Boyarsky, and O. Ruchayskiy, Hadronic decays of a light Higgs-like scalar, Phys. Rev. D99 (2019), no. 1 015019, [arXiv: 1806.07759 ]. 99

[295] KTeV Collaboration, A. Alavi-Harati et al., Search for the rare decay $K(L)-i$ piO $e+e-$, Phys. Rev. Lett. 93 (2004) 021805, [hep-ex/ 0309072 ]. 102

[296] S. Alekhin et al., A facility to Search for Hidden Particles at the CERN SPS: the SHiP physics case, Rept. Prog. Phys. 79 (2016), no. 12 124201, [arXiv:1504.04855]. 102

[297] J. L. Feng, I. Galon, F. Kling, and S. Trojanowski, Dark Higgs bosons at the ForwArd Search ExpeRiment, Phys. Rev. D97 (2018), no. 5055034 , [arXiv:1710.09387]. 102

[298] J. Beacham et al., Physics Beyond Colliders at CERN: Beyond the Standard Model Working Group Report, [arXiv:1901.09966]. 102

[299] SHiP Collaboration, C. Ahdida et al., Sensitivity of the SHiP experiment to scalar portal, 2019. In progress. 103 
[300] Belle-II Collaboration, W. Altmannshofer et al., The Belle II Physics Book, [arXiv:1808.10567]. 103

[301] K. Bondarenko, A. Boyarsky, M. Ovchynnikov, O. Ruchayskiy, and L. Shchutska, Probing new physics with displaced vertices: muon tracker at CMS, [arXiv:1903.11918]. 103

[302] CMS Collaboration, A. M. Sirunyan et al., Search for invisible decays of a Higgs boson produced through vector boson fusion in proton-proton collisions at $\sqrt{s}=$ $13 \mathrm{TeV}$, [arXiv:1809.05937]. 103

[303] F. An et al., Precision Higgs Physics at CEPC, [arXiv:1810.09037]. 104

[304] M. Cirelli, E. Del Nobile, and P. Panci, Tools for model-independent bounds in direct dark matter searches, JCAP 1310 (2013) 019, [arXiv : 1307 . 5955]. 104, 105

[305] F. Kahlhoefer, S. Kulkarni, and S. Wild, Exploring light mediators with low-threshold direct detection experiments, JCAP 1711 (2017), no. 11 016, [arXiv:1707.08571]. 105

[306] CRESST Collaboration, G. Angloher et al., Results on MeV-scale dark matter from a gram-scale cryogenic calorimeter operated above ground, Eur. Phys. J. C77 (2017), no. 9 637, [arXiv:1707.06749]. 106

[307] SuperCDMS Collaboration, R. Agnese et al., New Results from the Search for Low-Mass Weakly Interacting Massive Particles with the CDMS Low Ionization Threshold Experiment, Phys. Rev. Lett. 116 (2016), no. 7071301, [arXiv:1509.02448].

[308] DAMIC Collaboration, A. Aguilar-Arevalo et al., Search for low-mass WIMPs in a $0.6 \mathrm{~kg}$ day exposure of the DAMIC experiment at SNOLAB, Phys. Rev. D94 (2016), no. 8 082006, [arXiv:1607.07410]. 106

[309] R. E. Sanderson, J. L. Carlin, E. C. Cunningham, N. Garavito-Camargo, P. Guhathakurta, K. V. Johnston, C. F. P. Laporte, T. S. Li, and S. T. Sohn, The Multidimensional Milky Way, [arXiv:1903.07641]. 110

[310] R. Murgia, V. Iršič, and M. Viel, Novel constraints on noncold, nonthermal dark matter from Lyman- $\alpha$ forest data, Phys. Rev. D98 (2018), no. 8083540 , [arXiv:1806.08371]. 110

[311] P. Salucci, M. I. Wilkinson, M. G. Walker, G. F. Gilmore, E. K. Grebel, A. Koch, C. F. Martins, and R. F. G. Wyse, Dwarf spheroidal galaxy kinematics and spiral galaxy scaling laws, Mon. Not. Roy. Astron. Soc. 420 (2012) 2034, [arXiv:1111.1165]. 230 
[312] R. Kuzio de Naray, S. S. McGaugh, and W. J. G. de Blok, Mass Models for Low Surface Brightness Galaxies with High Resolution Optical Velocity Fields, Astrophys. J. 676 (2008) 920-943, [arXiv:0 0712 .0860]. 230

[313] M. Spano, M. Marcelin, P. Amram, C. Carignan, B. Epinat, and O. Hernandez, GHASP: An H-alpha kinematic survey of spiral and irregular galaxies. 5. Dark matter distribution in 36 nearby spiral galaxies, Mon. Not. Roy. Astron. Soc. 383 (2008) 297-316, [arXiv:0710.1345]. 230

[314] S.-H. Oh et al., High-resolution mass models of dwarf galaxies from LITTLE THINGS, Astron. J. 149 (2015) 180, [arXiv: 1502 . 01281]. 230 